\title{
Stereospecific Cross Couplings to Set Benzylic, All-Carbon Quaternary Stereocenters in High Enantiopurity
}

\author{
Qi Zhou, Kelsey M. Cobb, Tianyu Tan, Mary P. Watson* \\ Department of Chemistry and Biochemistry, University of Delaware, Newark, DE 19716 \\ mpwatson@udel.edu
}

\section{Supplementary Materials}

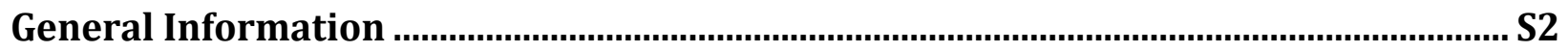

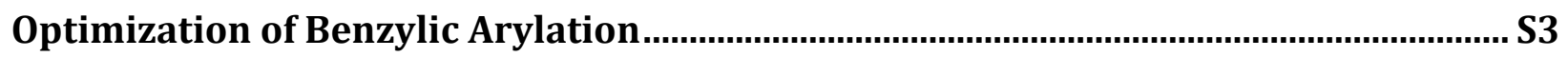

Stereospecific Arylation to Prepare Diaryl and Triarylalkanes .................................... S4

General Procedure A: Stereospecific Arylation of Tertiary Benzylic Acetates....................... S4

Preparation of Enantioenriched Tertiary Benzyl Acetates .......................................S14

General Procedure B: Preparation of (S)-2-(Naphthalen-2-yl)butan-2-yl Acetate (1a).....S14

Preparation of $(S, E)-2-($ Naphthalen-2-yl)-5-(o-tolyl)pent-4-en-2-yl acetate $((S)-1 \mathrm{~g})$........S18

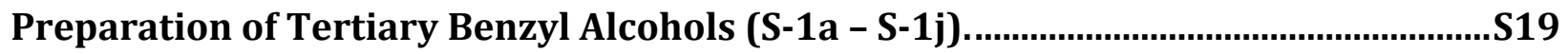

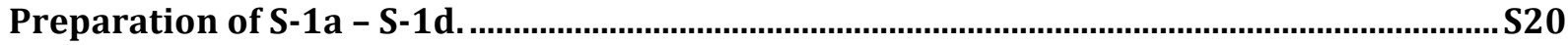

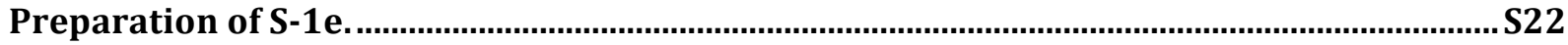

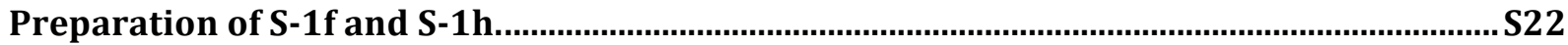

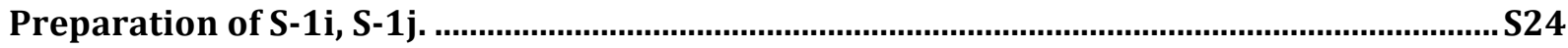

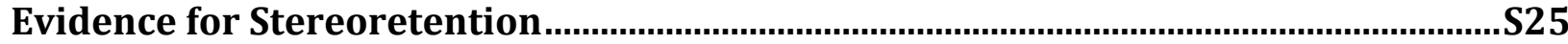

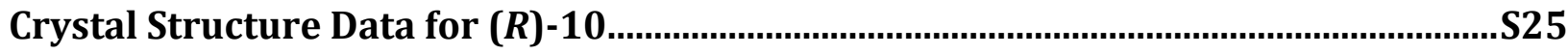

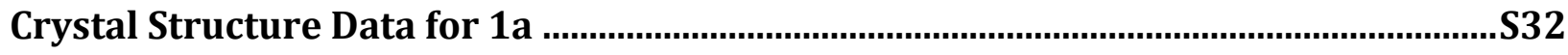

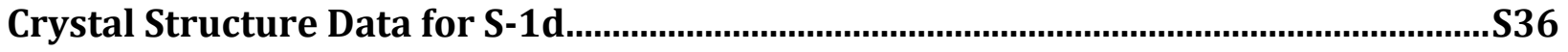

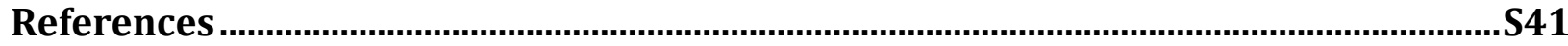

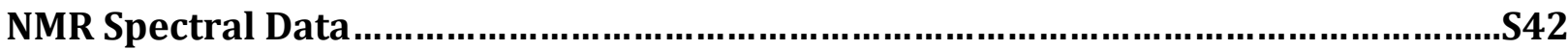

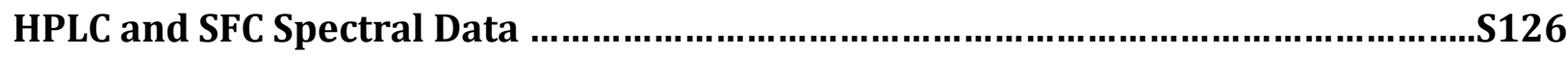




\section{General Information}

Reactions were performed in oven-dried vials with Teflon-lined caps or in oven-dried round-bottomed flasks unless otherwise noted. On occasions when a viscous mixture formed in the reaction vials, a higher speed of stirring or shaking was performed to guarantee sufficient mixing. Flasks were fitted with rubber septa, and reactions were conducted under a positive pressure of $\mathrm{N}_{2}$. Stainless steel syringes or cannulae were used to transfer air- and moisturesensitive liquids. Flash chromatography was performed on silica gel $60(40-63 \mu \mathrm{m}$, or 5-20 $\mu \mathrm{m}$ $60 \AA$ ) unless otherwise noted. Commercial reagents were purchased from Sigma Aldrich, Acros, Fisher, Strem, TCI, Combi Blocks, Alfa Aesar, or Cambridge Isotopes Laboratories and used as received with the following exceptions: sodium methoxide, anhydrous 2-methyltetrahydrofuran, diethyl zinc, dimethyl zinc $(1.0 \mathrm{M}$ in PhMe) were purchased from vendors and immediately placed in a $\mathrm{N}_{2}$-atmosphere glovebox for storage. Acetic anhydride and $\mathrm{Ti}(\mathrm{O}-i \mathrm{Pr})_{4}$ were distilled before use and stored under $\mathrm{N}_{2}$. Toluene, $\mathrm{CH}_{2} \mathrm{Cl}_{2}$, and THF were dried by passing through drying columns and stored over activated $4 \AA \mathrm{MS}$ in a $\mathrm{N}_{2}$-atmosphere glovebox. ${ }^{1}(R, R)$-Bis(sulfonamide) diol ligand $\mathbf{L 1}$ was prepared according to reported literature procedure. ${ }^{2}$ Bis(4-((tertbutyldimethylsilyl)oxy)butyl)zinc was prepared according to reported literature procedure and used immediately. ${ }^{3}$ Oven-dried potassium carbonate was added into $\mathrm{CDCl}_{3}$ to remove trace amount of acid. Proton nuclear magnetic resonance $\left({ }^{1} \mathrm{H}\right.$ NMR) spectra and carbon nuclear magnetic resonance $\left({ }^{13} \mathrm{C} \mathrm{NMR}\right)$ spectra were recorded on both $400 \mathrm{MHz}$ and $600 \mathrm{MHz}$ spectrometers. Chemical shifts for protons are reported in parts per million downfield from tetramethylsilane and are referenced to residual protium in the NMR solvent $\left(\mathrm{CHCl}_{3}=\delta 7.26\right)$. Chemical shifts for carbon are reported in parts per million downfield from tetramethylsilane and are referenced to the carbon resonances of the solvent $\left(\mathrm{CDCl}_{3}=\delta 77.2\right)$. Data are represented as follows: chemical shift, multiplicity $(\mathrm{br}=$ broad, $\mathrm{s}=$ singlet, $\mathrm{d}=$ doublet, $\mathrm{t}=$ triplet, $\mathrm{q}=$ quartet, $\mathrm{p}$ $=$ pentet, $\mathrm{m}=$ multiplet, $\mathrm{dd}=$ doublet of doublets, $\mathrm{h}=$ heptet $)$, coupling constants in Hertz $(\mathrm{Hz})$, integration. Infrared (IR) spectra were obtained using FTIR spectrophotometers with material loaded onto a $\mathrm{NaCl}$ plate. The mass spectral and X-ray crystallography data were obtained at the University of Delaware facilities. Optical rotations were measured using a $2.5 \mathrm{~mL}$ cell with a 0.1 $\mathrm{dm}$ path length. Melting points were taken on a Stuart SMP10 instrument. Enantiomeric excess (ee) was determined using chiral HPLC analysis at the University of Delaware or chiral SFC analysis at Lotus Separations, Inc.

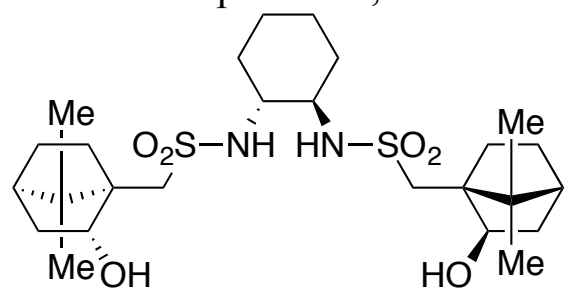

L1

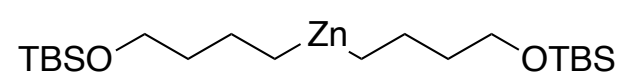

bis(4-((tert-butyldimethylsilyl)oxy)butyl)zinc 


\section{Optimization of Benzylic Arylation}

\section{Detailed Optimization Table}
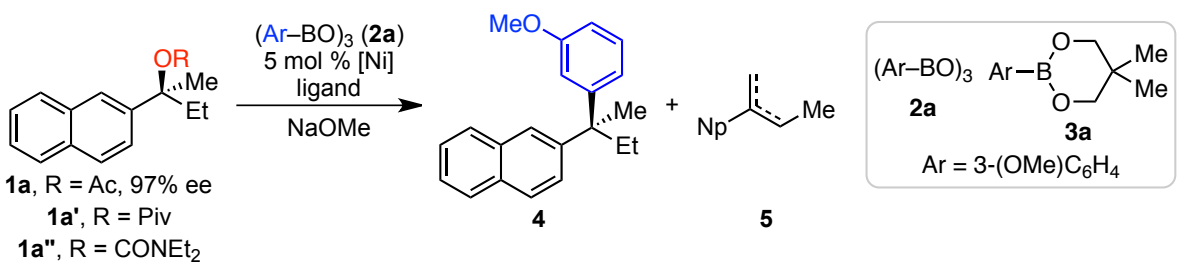

\begin{tabular}{|c|c|c|c|c|c|c|c|c|c|}
\hline \multirow[b]{2}{*}{ entry } & \multirow[b]{2}{*}[\mathrm{Ni}]{} & \multirow[b]{2}{*}{$\begin{array}{l}\text { ligand } \\
(\mathrm{mol} \%)\end{array}$} & \multirow[b]{2}{*}{$\begin{array}{l}\text { temp } \\
\left({ }^{\circ} \mathrm{C}\right) \\
\end{array}$} & \multirow[b]{2}{*}{ solvent } & \multirow[b]{2}{*}{$\begin{array}{l}\text { time } \\
\text { (h) }\end{array}$} & \multicolumn{2}{|c|}{ yield $(\%)^{a}$} & \multirow[b]{2}{*}{$\begin{array}{c}\text { ee of } 4 \\
(\%)^{b}\end{array}$} & \multirow[b]{2}{*}{ es $(\%)^{c}$} \\
\hline & & & & & & 4 & 5 & & \\
\hline 1 & $\mathrm{Ni}(\mathrm{cod})_{2}$ & none & 80 & $\mathrm{PhMe}$ & 2 & 93 & 2 & 20 & 21 \\
\hline 2 & $\mathrm{Ni}(\mathrm{cod})_{2}$ & $\mathrm{PhPCy}_{2}(11)$ & 80 & $\mathrm{PhMe}$ & 2 & 74 & 22 & 87 & 90 \\
\hline 3 & $\mathrm{Ni}(\mathrm{cod})_{2}$ & $\mathrm{PhPCy}_{2}(11)$ & 60 & $\mathrm{PhMe}$ & 5 & 72 & 25 & 90 & 93 \\
\hline 4 & $\mathrm{Ni}(\mathrm{cod})_{2}$ & $\mathrm{PhPCy}_{2}(11)$ & 60 & THF & 5 & 63 & 24 & 93 & 96 \\
\hline 5 & $\mathrm{Ni}(\mathrm{cod})_{2}$ & CyJohnPhos (11) & 40 & THF & 16 & 57 & 9 & 96 & 99 \\
\hline 6 & $\mathrm{Ni}(\operatorname{cod})_{2}$ & CyJohnPhos (5) & 40 & THF & 16 & 81 & 6 & 96 & 99 \\
\hline 7 & $\mathrm{Ni}(\mathrm{cod})_{2}$ & CyJohnPhos (5) & 40 & 1,4-dioxane & 39 & 97 & 5 & 97 & $>99$ \\
\hline 8 & $\mathrm{Ni}(\operatorname{cod})_{2}$ & CyJohnPhos (5) & 40 & 2-Me-THF & 22 & 92 & 8 & 96 & 99 \\
\hline 9 & $\mathrm{NiCl}_{2} \cdot \mathrm{DME}$ & CyJohnPhos (5) & 40 & 2-Me-THF & 22 & 90 & 6 & 95 & 98 \\
\hline $10^{d}$ & $\mathrm{NiCl}_{2} \cdot \mathrm{DME}$ & CyJohnPhos (5) & 40 & 2-Me-THF & 22 & 99 & $\leq 3$ & 97 & $>99$ \\
\hline
\end{tabular}

Conditions: 1a $(0.10 \mathrm{mmol}), \mathbf{2 a}(1.0$ equiv), [Ni] (5 mol \%), ligand, $\mathrm{NaOMe}(2.0$ equiv), solvent (0.4 M), unless otherwise noted. ${ }^{a}$ Determined by ${ }^{1} \mathrm{H}$ NMR analysis using an internal standard. Total yields over $100 \%$ reflect the inherent error bar of ${ }^{1} \mathrm{H}$ NMR yields, particularly for minor products. ${ }^{b}$ Determined by HPLC analysis using a chiral stationary phase. ${ }^{c}$ es $=$ enantiospecificity $=\left(\mathrm{ee}_{\text {product }}\right) /\left(\mathrm{ee}_{\text {starting material }}\right) .{ }^{d}$ Boronate ester 3a used in place of boroxine 2a.

\section{Evaluation of Aryl Boronate Reagents}
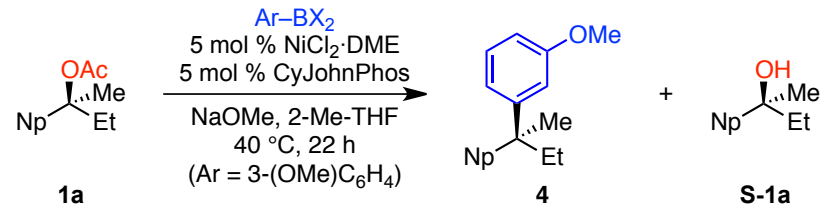

\begin{tabular}{|c|c|c|c|c|c|c|}
\hline \multirow[b]{2}{*}{ Entry } & \multirow[b]{2}{*}{$\mathrm{Ar}-\mathrm{BX}_{2}$ (equiv) } & \multicolumn{2}{|c|}{ yield $(\%)^{a}$} & & \multirow[b]{2}{*}{ es $(\%)^{c}$} \\
\hline & & 4 & S-1a & ee of $1 \mathbf{a}(\%)^{b}$ & ee of $4(\%)^{b}$ & \\
\hline 1 & $(\mathrm{ArBO})_{3}(\mathbf{2 a})(1.0)$ & 90 & - & 97 & 95 & 98 \\
\hline 2 & $\begin{array}{c}3 \mathbf{a} \\
(2.0)\end{array}$ & 99 & 一 & 97 & 97 & $>99$ \\
\hline 3 & $\mathrm{Ar}-\mathrm{B}(\mathrm{OH})_{2}(2.0)$ & 86 & 5 & 94 & 92 & 98 \\
\hline 4 & Ar-Bpin (2.0) & 80 & 0 & 94 & 92 & 98 \\
\hline 5 & $\mathrm{Ar}-\mathrm{BF}_{3} \mathrm{~K}(2.0)$ & 0 & 90 & 94 & - & - \\
\hline
\end{tabular}

Conditions: 1a (0.10 mmol), $\mathrm{ArBX}_{2}, \mathrm{NiCl}_{2} \cdot \mathrm{DME}(5 \mathrm{~mol} \%)$, CyJohnPhos (5 mol \%), NaOMe (2.0 equiv), 2-MeTHF (0.4 M), $40{ }^{\circ} \mathrm{C}, 22 \mathrm{~h} .{ }^{a}$ Determined by ${ }^{1} \mathrm{H}$ NMR analysis using an internal standard. Total yields over $100 \%$ 
reflect the inherent error bar of ${ }^{1} \mathrm{H}$ NMR yields, particularly for minor products. ${ }^{b}$ Determined by HPLC analysis using a chiral stationary phase. ${ }^{c}$ es $=$ enantiospecificity $=\left(\mathrm{e}_{\text {product }}\right) /\left(\mathrm{ee}_{\text {starting material }}\right)$.

\section{Stereospecific Arylation to Prepare Diaryl and Triarylalkanes}

\section{General Procedure A: Stereospecific Arylation of Tertiary Benzylic Acetates}

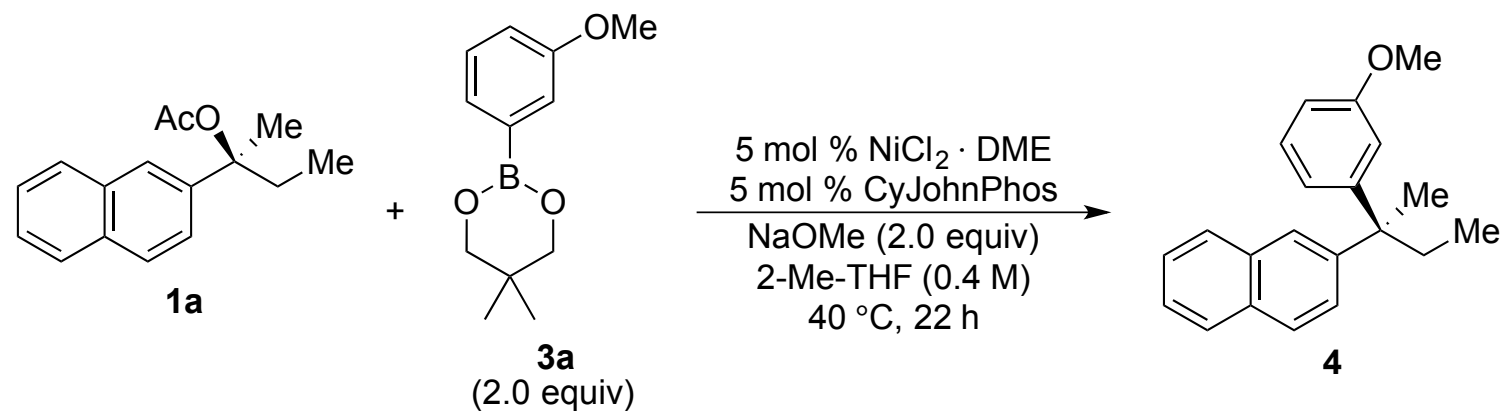

(S)-2-(2-(3-Methoxyphenyl)butan-2-yl)naphthalene ((S)-4). In a $\mathrm{N}_{2}$-atmosphere glovebox, $\mathrm{NiCl}_{2} \cdot \mathrm{DME}$ (4.4 mg, $0.020 \mathrm{mmol}, 5 \mathrm{~mol} \%$ ), CyJohnPhos (7.0 mg, $0.020 \mathrm{mmol}, 5 \mathrm{~mol}$ $\%$ ) and $\mathrm{NaOMe}$ (43 mg, $0.80 \mathrm{mmol}, 2.0$ equiv) were weighed into a 1-dram vial fitted with a magnetic stir bar. 2-(3-Methoxyphenyl)-5,5-dimethyl-1,3,2-dioxaborinane (3a, $176 \mathrm{mg}, 0.800$ mmol, 2.0 equiv) and (S)-2-(naphthalen-2-yl)butan-2-yl acetate (1a, prepared in 95\% ee, $97 \mathrm{mg}$, $0.40 \mathrm{mmol}, 1.0$ equiv) were added, followed by $2-\mathrm{Me}-\mathrm{THF}(1.0 \mathrm{~mL}, 0.4 \mathrm{M})$. The vial was capped with a Teflon-lined cap and removed from the glovebox. The mixture was stirred at 40 ${ }^{\circ} \mathrm{C}$ for $22 \mathrm{~h}$. Please note that these reactions are heterogeneous and vigorous stirring is critical. The reaction mixture was then diluted with $\mathrm{Et}_{2} \mathrm{O}(5 \mathrm{~mL})$ and filtered through a plug of silica gel and Celite ${ }^{\circledR}$, which was then rinsed with $\mathrm{Et}_{2} \mathrm{O}(\sim 15 \mathrm{~mL})$. The filtrate was concentrated and then purified by silica gel chromatography $\left(0-2 \% \mathrm{Et}_{2} \mathrm{O} /\right.$ hexanes) to give the compound 4 (run 1: $100.3 \mathrm{mg}, 86 \%$; run 2: $105.7 \mathrm{mg}, 91 \%$ ) as a colorless sticky oil. The enantiomeric excess was determined to be $93 \%$ (run 1: $93 \%$ ee; run 2: 92\% ee) by chiral HPLC analysis (CHIRALPAK $\mathrm{IB}, 0.6 \mathrm{~mL} / \mathrm{min}, 0.1 \% i-\mathrm{PrOH} / \mathrm{h}$ exane, $\lambda=254 \mathrm{~nm}) ; \mathrm{t}_{\mathrm{R}}$ (major) $=12.058 \mathrm{~min}, \mathrm{t}_{\mathrm{R}}($ minor $)=14.930$ $\min .[\alpha]_{\mathrm{D}}{ }^{24}=-10.2^{\circ}\left(\mathrm{c} 4.25, \mathrm{CHCl}_{3}\right):{ }^{1} \mathrm{H}$ NMR $\left(600 \mathrm{MHz}, \mathrm{CDCl}_{3}\right) \delta 7.85-7.81(\mathrm{~m}, 1 \mathrm{H}), 7.81-$ $7.77(\mathrm{~m}, 2 \mathrm{H}), 7.69(\mathrm{~d}, J=8.7 \mathrm{~Hz}, 1 \mathrm{H}), 7.49-7.42(\mathrm{~m}, 2 \mathrm{H}), 7.21-7.16(\mathrm{~m}, 2 \mathrm{H}), 6.83-6.79(\mathrm{~m}$, $2 \mathrm{H}), 6.76-6.69(\mathrm{~m}, 1 \mathrm{H}), 3.75(\mathrm{~s}, 3 \mathrm{H}), 2.31-2.20(\mathrm{~m}, 2 \mathrm{H}), 1.70(\mathrm{~s}, 3 \mathrm{H}), 0.77(\mathrm{t}, J=7.3 \mathrm{~Hz}, 3 \mathrm{H})$; ${ }^{13} \mathrm{C}$ NMR $\left(151 \mathrm{MHz}, \mathrm{CDCl}_{3}\right) \delta 159.4,151.5,146.9,133.3,131.9,128.9,128.1,127.6,127.5$, $127.2,125.9,125.5,124.9,120.4,114.3,110.3,55.3,46.8,33.9,26.9,9.4$; FTIR (NaCl/thin film) 3054, 2967, 2934, 2877, 1599, 1582, 1485, 1457, 1430, 1290, 1254, 1053, 819, 749, 703, 477 $\mathrm{cm}^{-1}$; HRMS (CI+) $[\mathrm{M}+\mathrm{H}]^{+}$calculated for $\mathrm{C}_{21} \mathrm{H}_{23} \mathrm{O}: 291.1749$, found: 291.1773.

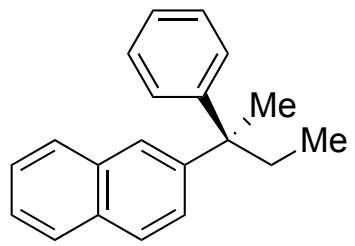

(R)-2-(2-Phenylbutan-2-yl)naphthalene ((R)-6). Prepared via General Procedure A using 1a (prepared in 96\% ee) as a colorless oil (run 1: $100 \mathrm{mg}, 96 \%$; run 2: $96.8 \mathrm{mg}$, 93\%). The enantiomeric excess was determined to be $95 \%$ (run 1: $95 \%$ ee; run 2: $95 \%$ ee) by chiral SFC 
analysis (CHIRALCEL OJ-H $(25 \times 0.46 \mathrm{~cm}), 3.0 \mathrm{~mL} / \mathrm{min}, 15 \% \mathrm{EtOH}(0.1 \%$ diethylamine $) / \mathrm{CO}_{2}$ $(100$ bar $), \lambda=220 \mathrm{~nm}) ; \mathrm{t}_{\mathrm{R}}($ major $)=6.25 \mathrm{~min}, \mathrm{t}_{\mathrm{R}}($ minor $)=7.32 \mathrm{~min} .[\alpha]_{\mathrm{D}}{ }^{24}=+13.3^{\circ}(\mathrm{c} 1.02$, $\left.\mathrm{CHCl}_{3}\right):{ }^{1} \mathrm{H}$ NMR $\left(400 \mathrm{MHz}, \mathrm{CDCl}_{3}\right) \delta 7.88-7.78(\mathrm{~m}, 3 \mathrm{H}), 7.71(\mathrm{~d}, J=8.7 \mathrm{~Hz}, 1 \mathrm{H}), 7.53-$ $7.43(\mathrm{~m}, 2 \mathrm{H}), 7.33-7.18(\mathrm{~m}, 6 \mathrm{H}), 2.36-2.22(\mathrm{~m}, 2 \mathrm{H}), 1.73(\mathrm{~s}, 3 \mathrm{H}), 0.79(\mathrm{t}, J=7.4 \mathrm{~Hz}, 3 \mathrm{H}) ;{ }^{13} \mathrm{C}$ NMR $\left(101 \mathrm{MHz}, \mathrm{CDCl}_{3}\right) \delta 149.7,147.0,133.2,131.8,128.10,128.07,127.63,127.61,127.5$, 127.3, 126.0, 125.7, 125.6, 124.9, 46.8, 33.8, 26.9, 9.4; FTIR (NaCl/thin film) 3055, 2968, 2934, 2876, 1599, 1494, 1444, 1380, 1273, 1131, 1029, 948, 897, $770 \mathrm{~cm}^{-1}$; HRMS (EI+) [M]+ calculated for $\mathrm{C}_{20} \mathrm{H}_{20}: 260.1565$, found: 260.1558 .

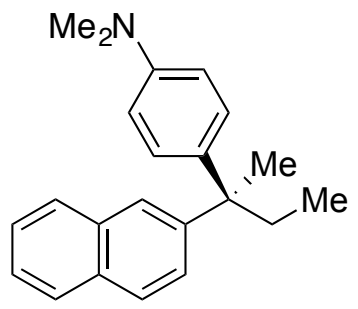

$(R)-N, N$-Dimethyl-4-(2-(naphthalen-2-yl)butan-2-yl)aniline ((R)-7). Prepared via General Procedure A using 1a (prepared in 96\% ee) as a white solid (mp 64-66 ${ }^{\circ} \mathrm{C} ; 99 \mathrm{mg}$, 82\%). The enantiomeric excess was determined to be $96 \%$ by chiral SFC analysis (CHIRALCEL OJ-H (25 x $0.46 \mathrm{~cm}), 3.5 \mathrm{~mL} / \mathrm{min}, 55 \% \mathrm{MeOH}(0.1 \%$ diethylamine $\left.) / \mathrm{CO}_{2}(100 \mathrm{bar}), \lambda=220 \mathrm{~nm}\right) ; \mathrm{t}_{\mathrm{R}}($ major $)$ $=11.68 \mathrm{~min}, \mathrm{t}_{\mathrm{R}}$ (minor) $=17.76 \mathrm{~min} .[\alpha]_{\mathrm{D}}{ }^{24}=+22.6^{\circ}\left(\mathrm{c} \mathrm{3.8}, \mathrm{CHCl}_{3}\right):{ }^{1} \mathrm{H} \mathrm{NMR}\left(600 \mathrm{MHz}, \mathrm{CDCl}_{3}\right)$ $\delta 7.81(\mathrm{dd}, J=8.0,1.3 \mathrm{~Hz}, 1 \mathrm{H}), 7.79-7.74(\mathrm{~m}, 2 \mathrm{H}), 7.67(\mathrm{~d}, J=8.7 \mathrm{~Hz}, 1 \mathrm{H}), 7.48-7.38(\mathrm{~m}$, 2H), $7.21(\mathrm{dd}, J=8.7,1.9 \mathrm{~Hz}, 1 \mathrm{H}), 7.11-7.05(\mathrm{~m}, 2 \mathrm{H}), 6.68-6.63(\mathrm{~m}, 2 \mathrm{H}), 2.91(\mathrm{~s}, 6 \mathrm{H}), 2.28-$ $2.15(\mathrm{~m}, 2 \mathrm{H}), 1.67(\mathrm{~s}, 3 \mathrm{H}), 0.76(\mathrm{t}, J=7.3 \mathrm{~Hz}, 3 \mathrm{H}) ;{ }^{13} \mathrm{C}$ NMR $\left(151 \mathrm{MHz}, \mathrm{CDCl}_{3}\right) \delta 148.6,147.6$, $137.8,133.3,131.8,128.2,128.1,127.50,127.46,127.4,125.8,125.4,124.8,112.3,45.9,40.8$, 34.0, 27.0, 9.4; FTIR (NaCl/thin film) 3431, 3054, 2966, 2934, 2876, 1613, 1519, 1444, 1348, 1201, 1166, 948, 818, 746, 569, $476 \mathrm{~cm}^{-1}$; HRMS (LIFDI) [M]+ calculated for $\mathrm{C}_{22} \mathrm{H}_{25} \mathrm{~N}$ : 303.1987, found: 303.1966 .

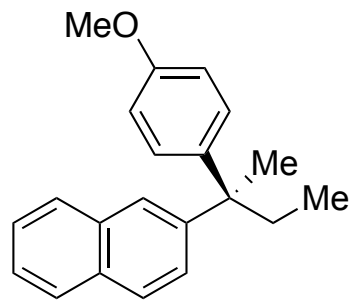

(R)-2-(2-(4-Methoxyphenyl)butan-2-yl)naphthalene $((\boldsymbol{R})-8)$. Prepared via General Procedure A using 1a (prepared in 96\% ee) as a colorless oil (run 1: $110 \mathrm{mg}, 95 \%$; run 2: $105.7 \mathrm{mg}, 91 \%$ ). The enantiomeric excess was determined to be $96 \%$ (run 1: $96 \%$ ee; run 2: $96 \%$ ee) by chiral SFC analysis (CHIRALCEL OJ-H $(25 \mathrm{x} \quad 0.46 \mathrm{~cm}), \quad 3.0 \mathrm{~mL} / \mathrm{min}, \quad 25 \% \quad i-\mathrm{PrOH}(0.1 \%$ diethylamine $\left.) / \mathrm{CO}_{2}(100 \mathrm{bar}), \lambda=220 \mathrm{~nm}\right) ; \mathrm{t}_{\mathrm{R}}($ major $)=4.89 \mathrm{~min}, \mathrm{t}_{\mathrm{R}}($ minor $)=6.27 \mathrm{~min} .[\alpha]_{\mathrm{D}}{ }^{24}=$ $+12.4^{\circ}\left(\mathrm{c} 0.98, \mathrm{CHCl}_{3}\right):{ }^{1} \mathrm{H} \mathrm{NMR}\left(400 \mathrm{MHz}, \mathrm{CDCl}_{3}\right) \delta 7.89-7.83(\mathrm{~m}, 1 \mathrm{H}), 7.83-7.77(\mathrm{~m}, 2 \mathrm{H})$, $7.71(\mathrm{~d}, J=8.7 \mathrm{~Hz}, 1 \mathrm{H}), 7.54-7.43(\mathrm{~m}, 2 \mathrm{H}), 7.23-7.13(\mathrm{~m}, 3 \mathrm{H}), 6.87-6.80(\mathrm{~m}, 2 \mathrm{H}), 3.81(\mathrm{~s}$, $3 \mathrm{H}), 2.35-2.16(\mathrm{~m}, 2 \mathrm{H}), 1.71(\mathrm{~s}, 3 \mathrm{H}), 0.79(\mathrm{t}, J=7.3 \mathrm{~Hz}, 3 \mathrm{H}) ;{ }^{13} \mathrm{C} \mathrm{NMR}\left(101 \mathrm{MHz}, \mathrm{CDCl}_{3}\right) \delta$ $157.5,147.2$, 141.8, 133.2, 131.8, 128.6, 128.1, 127.6, 127.5, 127.3, 125.9, 125.5, 124.8, 113.3, 55.3, 46.1, 34.0, 27.1, 9.4; FTIR (NaCl/thin film) 3055, 2967, 2932, 2876, 1511, 1463, 1441, $1298,1248,1182,1034,852,745 \mathrm{~cm}^{-1}$; HRMS $(\mathrm{CI}+)[\mathrm{M}+\mathrm{H}]^{+}$calculated for $\mathrm{C}_{21} \mathrm{H}_{23} \mathrm{O}: 291.1749$, found: 291.1768 . 


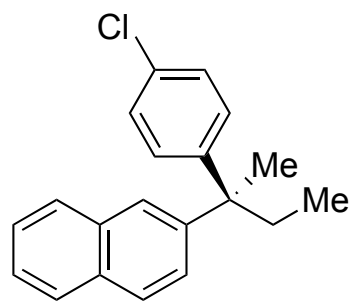

(R)-2-(2-(4-Chlorophenyl)butan-2-yl)naphthalene ((R)-9). Prepared via General Procedure A using 1a (prepared in 95\% ee) as a colorless oil (run 1: $62.1 \mathrm{mg}, 53 \%$; run 2: $70.4 \mathrm{mg}, 60 \%$ ). The enantiomeric excess was determined to be $92 \%$ (run 1: $92 \%$ ee; run 2: $92 \%$ ee) by chiral SFC analysis (CHIRALCEL OJ-H $(25 \times 0.46 \mathrm{~cm}), 3.0 \mathrm{~mL} / \mathrm{min}, 15 \% \mathrm{EtOH}(0.1 \%$ diethylamine $) / \mathrm{CO}_{2}$ $(100$ bar), $\lambda=220 \mathrm{~nm}) ; \mathrm{t}_{\mathrm{R}}$ (major) $=6.18 \mathrm{~min}, \mathrm{t}_{\mathrm{R}}($ minor $)=6.97 \mathrm{~min} .[\alpha]_{\mathrm{D}}{ }^{24}=+10.8^{\circ}(\mathrm{c} 1.66$, $\left.\mathrm{CHCl}_{3}\right):{ }^{1} \mathrm{H} \mathrm{NMR}\left(600 \mathrm{MHz}, \mathrm{CDCl}_{3}\right) \delta 7.86(\mathrm{~d}, J=8.0 \mathrm{~Hz}, 1 \mathrm{H}), 7.85-7.79(\mathrm{~m}, 2 \mathrm{H}), 7.73(\mathrm{~d}, J=$ $8.7 \mathrm{~Hz}, 1 \mathrm{H}), 7.55-7.45(\mathrm{~m}, 2 \mathrm{H}), 7.31-7.25(\mathrm{~m}, 2 \mathrm{H}), 7.20-7.13(\mathrm{~m}, 3 \mathrm{H}), 2.35-2.19(\mathrm{~m}, 2 \mathrm{H})$, $1.72(\mathrm{~s}, 3 \mathrm{H}), 0.80(\mathrm{t}, J=7.4 \mathrm{~Hz}, 3 \mathrm{H}) ;{ }^{13} \mathrm{C} \mathrm{NMR}\left(151 \mathrm{MHz}, \mathrm{CDCl}_{3}\right) \delta 148.3,146.4,133.3,131.9$, 131.6, 129.1, 128.2, 128.1, 127.8, 127.5, 127.0, 126.1, 125.7, 125.0, 46.6, 33.9, 26.9, 9.3; FTIR ( $\mathrm{NaCl} /$ thin film) 3055, 2969, 2934, 2887, 1599, 1489, 1092, 1012, 817, 746, $477 \mathrm{~cm}^{-1}$; HRMS $(\mathrm{EI}+)[\mathrm{M}]+$ calculated for $\mathrm{C}_{20} \mathrm{H}_{19} \mathrm{Cl} 294.1775$, found: 294.1189 .<smiles>CCNC(=O)c1ccc(C(C)(C)c2ccc3ccccc3c2)cc1</smiles>

$(R)-N, N$-Diethyl-4-(2-(naphthalen-2-yl)butan-2-yl)benzamide ((R)-10). Prepared via General Procedure A using 1a (prepared in $96 \%$ ee) except that the reaction mixture was heated at $60{ }^{\circ} \mathrm{C}$ for $12 \mathrm{~h}$. Compound 10 was obtained as white solid $\left(\mathrm{mp} 96-100{ }^{\circ} \mathrm{C}\right.$; run 1: $125 \mathrm{mg}$, 87\%; run 2: $122 \mathrm{mg}, 85 \%$ ). The enantiomeric excess was determined to be 94\% (run 1: 94\% ee, run 2: 94\% ee) by chiral HPLC analysis (CHIRALPAK IA, $0.8 \mathrm{~mL} / \mathrm{min}, 8 \% i$-PrOH/hexane, $\lambda=254 \mathrm{~nm}$ ); $\mathrm{t}_{\mathrm{R}}($ major $)=11.038 \mathrm{~min}, \mathrm{t}_{\mathrm{R}}($ minor $)=10.179 \mathrm{~min} .[\alpha]_{\mathrm{D}}{ }^{24}=+18.9^{\circ}\left(\mathrm{c} 1.16, \mathrm{CHCl}_{3}\right):{ }^{1} \mathrm{H} \mathrm{NMR}(600$ $\left.\mathrm{MHz}, \mathrm{CDCl}_{3}\right) \delta 7.82(\mathrm{~d}, J=8.0 \mathrm{~Hz}, 1 \mathrm{H}), 7.80-7.76(\mathrm{~m}, 2 \mathrm{H}), 7.69(\mathrm{~d}, J=8.7 \mathrm{~Hz}, 1 \mathrm{H}), 7.50-$ $7.42(\mathrm{~m}, 2 \mathrm{H}), 7.29(\mathrm{~d}, J=8.1 \mathrm{~Hz}, 2 \mathrm{H}), 7.24(\mathrm{~d}, J=8.4 \mathrm{~Hz}, 2 \mathrm{H}), 7.15(\mathrm{dd}, J=8.7,1.9 \mathrm{~Hz}, 1 \mathrm{H})$, 3.54 (br s, 2H), 3.28 (br s, 2H), $2.33-2.18$ (m, $J=7.1 \mathrm{~Hz}, 2 \mathrm{H}), 1.70$ (s, 3H), 1.23 (br s, 3H), 1.12 (br s, 3H), 0.77 (t, $J=7.3 \mathrm{~Hz}, 3 \mathrm{H}) ;{ }^{13} \mathrm{C} \mathrm{NMR}\left(151 \mathrm{MHz}, \mathrm{CDCl}_{3}\right) \delta 171.5,150.9,146.5$, $134.6,133.2,131.9,128.1,127.7,127.6,127.5,127.2,126.3,126.0,125.7,125.1,46.9,43.4$, 39.3, 33.8, 26.9, 14.4, 13.0, 9.3; FTIR (NaCl/thin film) 3053, 2970, 2934, 2876, 1630, 1457, 1424, 1288, 1098, 1019, 819, 748, $478 \mathrm{~cm}^{-1}$; HRMS $(\mathrm{CI}+)[\mathrm{M}+\mathrm{H}]^{+}$calculated for $\mathrm{C}_{25} \mathrm{H}_{30} \mathrm{NO}$ : 360.2327, found: 360.2347 .

X-ray quality crystals were obtained from slow evaporation of $\mathbf{1 0}$ in EtOAc. The crystal structure demonstrates that the absolute configuration is $R$ (Figure $\mathrm{S} 1$ ). The enantiomeric excess of the crystal was determined to be $96 \%$ ee by chiral HPLC analysis, with the major enantiomer matching that of the bulk material isolated as described above. 
Figure S1. Molecular diagram of $(R)$-10 with ellipsoids at 50\% probability. H-atoms omitted for clarity. (CCDC 1424635)
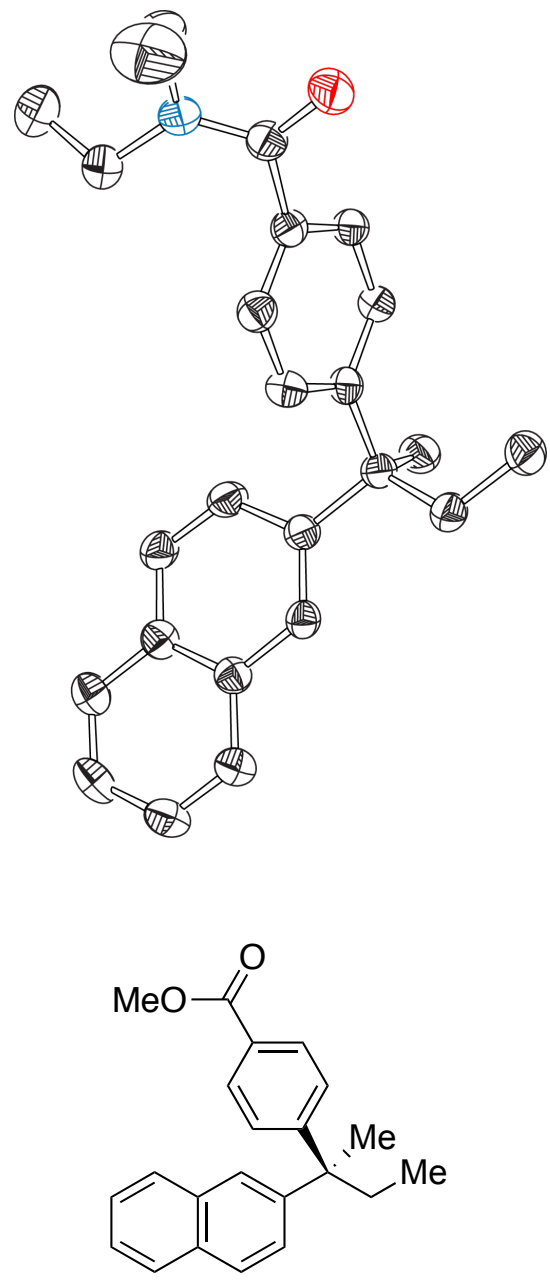

(R)-Methyl-4-(2-(naphthalen-2-yl)butan-2-yl)benzoate $((\boldsymbol{R})-11)$. Prepared via General Procedure A using 1a (prepared in 96\% ee) except that 3.0 equiv of 3 were used and the reaction mixture was heated at $60{ }^{\circ} \mathrm{C}$ for $12 \mathrm{~h}$. Compound 11 was obtained as a colorless oil (run 1: 114.5 mg, 90\%; run 2: $105.6 \mathrm{mg}, 83 \%$ ). The enantiomeric excess was determined to be $96 \%$ (run 1: 96\% ee, run 2: 96\% ee) by chiral HPLC analysis (CHIRALPAK IC, $0.6 \mathrm{~mL} / \mathrm{min}, 1 \% i$ $\mathrm{PrOH} /$ hexane, $\lambda=254 \mathrm{~nm}) ; \mathrm{t}_{\mathrm{R}}$ (major) $=30.604 \mathrm{~min}, \mathrm{t}_{\mathrm{R}}($ minor $)=33.299 \mathrm{~min}$. $[\alpha]_{\mathrm{D}}{ }^{24}=+8.1^{\circ}(\mathrm{c}$ 1.23, $\left.\mathrm{CHCl}_{3}\right):{ }^{1} \mathrm{H} \mathrm{NMR}\left(600 \mathrm{MHz}, \mathrm{CDCl}_{3}\right) \delta 7.98-7.90(\mathrm{~m}, 2 \mathrm{H}), 7.85-7.81(\mathrm{~m}, 1 \mathrm{H}), 7.81-$ $7.76(\mathrm{~m}, 2 \mathrm{H}), 7.69(\mathrm{~d}, J=8.7 \mathrm{~Hz}, 1 \mathrm{H}), 7.51-7.41(\mathrm{~m}, 2 \mathrm{H}), 7.34-7.28(\mathrm{~m}, 2 \mathrm{H}), 7.12(\mathrm{dd}, J=$ 8.6, 1.9 Hz, 1H), $3.90(\mathrm{~s}, 3 \mathrm{H}), 2.34-2.22(\mathrm{~m}, 2 \mathrm{H}), 1.72(\mathrm{~s}, 3 \mathrm{H}), 0.77(\mathrm{t}, J=7.4 \mathrm{~Hz}, 3 \mathrm{H}) ;{ }^{13} \mathrm{C}$ NMR $\left(151 \mathrm{MHz}, \mathrm{CDCl}_{3}\right) \delta 167.2,155.1,146.2,133.3,131.9,129.4,128.1,127.84,127.75$, 127.7, 127.6, 127.0, 126.1, 125.8, 125.0, 52.1, 47.2, 33.8, 26.8, 9.3; FTIR ( NaCl/thin film) 3055, 2969, 2878, 1718, 1608, 1435, 1279, 1188, 1115, 1018, 854, 819, 747, $477 \mathrm{~cm}^{-1}$; HRMS (CI+) $[\mathrm{M}+\mathrm{H}]^{+}$calculated for $\mathrm{C}_{22} \mathrm{H}_{23} \mathrm{O}_{2}: 319.1698$, found: 319.1708 . 


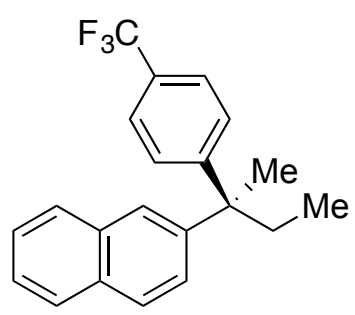

(R)-2-(2-(4-(Trifluoromethyl)phenyl)butan-2-yl)naphthalene ((R)-12). Prepared via General Procedure A using 1a (prepared in $96 \%$ ee) as a colorless oil (117 mg, 89\%). The enantiomeric excess was determined to be $96 \%$ by chiral HPLC analysis (CHIRALPAK IB, $0.2 \mathrm{~mL} / \mathrm{min}, 0 \% i$ $\mathrm{PrOH} /$ hexane, $\lambda=210 \mathrm{~nm}) ; \mathrm{t}_{\mathrm{R}}($ major $)=39.173 \mathrm{~min}, \mathrm{t}_{\mathrm{R}}($ minor $)=35.980 \mathrm{~min}$. A second run using 1 a (prepared in $95 \%$ ee) gave $12(95 \mathrm{mg}, 72 \%)$ in $95 \%$ ee. $[\alpha]_{\mathrm{D}}{ }^{24}=+9.9^{\circ}\left(\mathrm{c} 1.1, \mathrm{CHCl}_{3}\right):{ }^{1} \mathrm{H}$ NMR $\left(400 \mathrm{MHz}, \mathrm{CDCl}_{3}\right) \delta 7.91-7.84(\mathrm{~m}, 1 \mathrm{H}), 7.84-7.79(\mathrm{~m}, 2 \mathrm{H}), 7.73(\mathrm{~d}, J=8.6 \mathrm{~Hz}, 1 \mathrm{H})$, $7.58-7.45(\mathrm{~m}, 4 \mathrm{H}), 7.35(\mathrm{~d}, J=8.2 \mathrm{~Hz}, 2 \mathrm{H}), 7.13(\mathrm{dd}, J=8.7,1.9 \mathrm{~Hz}, 1 \mathrm{H}), 2.38-2.21(\mathrm{~m}, 2 \mathrm{H})$, $1.74(\mathrm{~s}, 3 \mathrm{H}), 0.79(\mathrm{t}, J=7.4 \mathrm{~Hz}, 3 \mathrm{H}) ;{ }^{13} \mathrm{C} \mathrm{NMR}\left(101 \mathrm{MHz}, \mathrm{CDCl}_{3}\right) \delta 153.9,145.9,133.2,131.9$, $128.1,128.02\left(\mathrm{q}, J_{C-F}=32.9 \mathrm{~Hz}\right), 128.0,127.9,127.6,126.9,126.2,125.8,125.1,125.0\left(\mathrm{q}, J_{C-F}\right.$ $=3.8 \mathrm{~Hz}), 124.4\left(\mathrm{q}, J_{C-F}=272.9 \mathrm{~Hz}\right), 47.0,33.8,26.8,9.25 ;{ }^{19} \mathrm{~F}$ NMR $\left(377 \mathrm{MHz}, \mathrm{CDCl}_{3}\right) \delta-$ 62.2; FTIR (NaCl/thin film) 3057, 2971, 2937, 2880, 1921, 1617, 1504, 1409, 1325, 1273, 1122 , 1068, 1016, 948, 841, $748 \mathrm{~cm}^{-1}$; HRMS (EI+) [M]+ calculated for $\mathrm{C}_{21} \mathrm{H}_{19} \mathrm{~F}_{3}$ : 328.1439, found: 328.1447 .

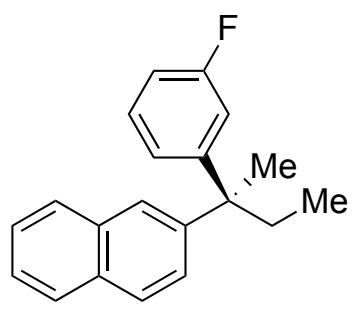

(S)-2-(2-(3-Fluorophenyl)butan-2-yl)naphthalene ((S)-13). Prepared via General Procedure A using 1a (prepared in 96\% ee) as a colorless oil (run 1: $103.5 \mathrm{mg}, 93 \%$; run 2: $106.4 \mathrm{mg}, 96 \%$ ). The enantiomeric excess was determined to be $94 \%$ (run 1: $94 \%$ ee; run 2: $94 \%$ ee) by chiral SFC analysis (CHIRALCEL OJ-H $(25 \mathrm{x} \quad 0.46 \mathrm{~cm}), \quad 3.0 \mathrm{~mL} / \mathrm{min}, \quad 8 \% \quad \mathrm{EtOH}(0.1 \%$ diethylamine $\left.) / \mathrm{CO}_{2}(100 \mathrm{bar}), \lambda=220 \mathrm{~nm}\right) ; \mathrm{t}_{\mathrm{R}}($ major $)=5.22 \mathrm{~min}, \mathrm{t}_{\mathrm{R}}($ minor $)=5.57 \mathrm{~min} .[\alpha]_{\mathrm{D}}{ }^{24}=$ $+7.5^{\circ}\left(\mathrm{c} 1.19, \mathrm{CHCl}_{3}\right):{ }^{1} \mathrm{H} \mathrm{NMR}\left(600 \mathrm{MHz}, \mathrm{CDCl}_{3}\right) \delta 7.83(\mathrm{~d}, J=7.9 \mathrm{~Hz}, 1 \mathrm{H}), 7.81-7.75(\mathrm{~m}$, 2H), $7.70(\mathrm{~d}, J=8.7 \mathrm{~Hz}, 1 \mathrm{H}), 7.52-7.41(\mathrm{~m}, 2 \mathrm{H}), 7.21(\mathrm{td}, J=8.0,6.3 \mathrm{~Hz}, 1 \mathrm{H}), 7.15(\mathrm{dd}, J=$ $8.7,1.9 \mathrm{~Hz}, 1 \mathrm{H}), 6.98(\mathrm{dt}, J=8.0,1.2 \mathrm{~Hz}, 1 \mathrm{H}), 6.95(\mathrm{dt}, J=11.1,2.2 \mathrm{~Hz}, 1 \mathrm{H}), 6.87(\mathrm{td}, J=8.5$, $2.7 \mathrm{~Hz}, 1 \mathrm{H}), 2.32-2.17(\mathrm{~m}, 2 \mathrm{H}), 1.70(\mathrm{~s}, 3 \mathrm{H}), 0.77(\mathrm{t}, J=7.3 \mathrm{~Hz}, 3 \mathrm{H}) ;{ }^{13} \mathrm{C} \mathrm{NMR}(151 \mathrm{MHz}$, $\left.\mathrm{CDCl}_{3}\right) \delta 162.9\left(\mathrm{~d}, J_{C-F}=245 \mathrm{~Hz}\right), 152.6\left(\mathrm{~d}, J_{C-F}=6.4 \mathrm{~Hz}\right), 146.3,133.3,131.9,129.4\left(\mathrm{~d}, J_{C-F}=\right.$ $8.2 \mathrm{~Hz}), 128.1,127.8,127.5,127.0,126.1,125.7,125.0,123.4\left(\mathrm{~d}, J_{C-F}=2.7 \mathrm{~Hz}\right), 114.7\left(\mathrm{~d}, J_{C-F}\right.$ $=21.7 \mathrm{~Hz}), 112.7\left(\mathrm{~d}, J_{C-F}=21.2 \mathrm{~Hz}\right), 46.8\left(\mathrm{~d}, J_{C-F}=1.5 \mathrm{~Hz}\right), 33.8,26.8,9.3 ;{ }^{19} \mathrm{~F}$ NMR $(565$ $\left.\mathrm{MHz}, \mathrm{CDCl}_{3}\right) \delta-113.6$; FTIR (NaCl/thin film) 3056, 2970, 2878, 1612, 1585, 1485, 1433, 1243 , $1163,917,818,783,477 \mathrm{~cm}^{-1}$; HRMS (EI+) [M]+ calculated for $\mathrm{C}_{20} \mathrm{H}_{19} \mathrm{~F}$ : 278.1471, found: 278.1479 . 


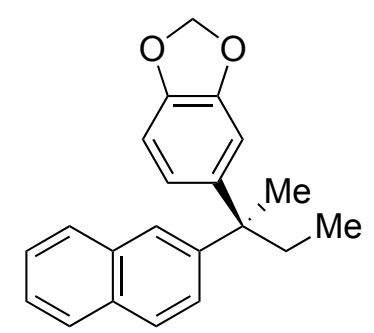

(S)-5-(2-(Naphthalen-2-yl)butan-2-yl)benzo-[1,3]-dioxole ((S)-14). Prepared via General Procedure A using 1a (prepared in 95\% ee) as a colorless oil (run 1: $110 \mathrm{mg}, 90 \%$; run 2: 100 $\mathrm{mg}, 82 \%$ ). The enantiomeric excess was determined to be $92 \%$ (run 1: $92 \%$ ee; run 2: $92 \%$ ee) by chiral SFC analysis (CHIRALCEL OJ-H $(25 \times 0.46 \mathrm{~cm}), 3.0 \mathrm{~mL} / \mathrm{min}, 30 \% \mathrm{EtOH}(0.1 \%$ diethylamine $\left.) / \mathrm{CO}_{2}(100 \mathrm{bar}), \lambda=220 \mathrm{~nm}\right) ; \mathrm{t}_{\mathrm{R}}($ major $)=4.19 \mathrm{~min}, \mathrm{t}_{\mathrm{R}}($ minor $)=4.93 \mathrm{~min} .[\alpha]_{\mathrm{D}}{ }^{24}=$ $+3.9^{\circ}\left(\mathrm{c} 4.57, \mathrm{CHCl}_{3}\right):{ }^{1} \mathrm{H}$ NMR $\left(600 \mathrm{MHz}, \mathrm{CDCl}_{3}\right) \delta 7.83(\mathrm{dd}, J=8.0,1.5 \mathrm{~Hz}, 1 \mathrm{H}), 7.81-7.77$ $(\mathrm{m}, 2 \mathrm{H}), 7.70(\mathrm{~d}, J=8.7 \mathrm{~Hz}, 1 \mathrm{H}), 7.50-7.41(\mathrm{~m}, 2 \mathrm{H}), 7.19(\mathrm{dd}, J=8.7,1.9 \mathrm{~Hz}, 1 \mathrm{H}), 6.74(\mathrm{~d}, J$ $=1.3 \mathrm{~Hz}, 2 \mathrm{H}), 6.70-6.65(\mathrm{~m}, 1 \mathrm{H}), 5.91(\mathrm{~s}, 2 \mathrm{H}), 2.31-2.14(\mathrm{~m}, 2 \mathrm{H}), 1.67(\mathrm{~s}, 3 \mathrm{H}), 0.78(\mathrm{t}, J=$ $7.3 \mathrm{~Hz}, 3 \mathrm{H}) ;{ }^{13} \mathrm{C}$ NMR $\left(151 \mathrm{MHz}, \mathrm{CDCl}_{3}\right) \delta 147.6,147.1,145.4,143.8,133.3,131.9,128.1$, 127.7, 127.5, 127.1, 126.0, 125.6, 124.8, 120.4, 108.7, 107.6, 100.9, 46.6, 34.1, 27.1, 9.4; FTIR (NaCl/thin film) $3055,2968,2933,2877,1631,1599,1485,1430,1235,1039,938,817,746 \mathrm{~cm}^{-}$ ${ }^{1}$; HRMS (EI+) [M]+ calculated for $\mathrm{C}_{21} \mathrm{H}_{20} \mathrm{O}: 304.1463$ found: 304.1482 .<smiles>CCC(c1ccccc1)(c1ccc2ccccc2c1)c1ccc(C)cc1C</smiles>

(S)-2-(2-(2,4-Dimethylphenyl)butan-2-yl)naphthalene ((S)-15). Prepared via General Procedure A using 1a (prepared in 96\% ee) as a colorless oil (run 1: $103.8 \mathrm{mg}, 90 \%$; run 2: 100.4 $\mathrm{mg}, 87 \%$ ). The enantiomeric excess was determined to be $95 \%$ (run 1: $95 \%$ ee; run 2: $95 \%$ ee) by chiral SFC analysis (CHIRALCEL OJ-H $(25 \times 0.46 \mathrm{~cm}), 3.0 \mathrm{~mL} / \mathrm{min}, 8 \% \mathrm{EtOH}(0.1 \%$ diethylamine $\left.) / \mathrm{CO}_{2}(100 \mathrm{bar}), \lambda=220 \mathrm{~nm}\right) ; \mathrm{t}_{\mathrm{R}}($ major $)=7.83 \mathrm{~min}, \mathrm{t}_{\mathrm{R}}($ minor $)=8.59 \mathrm{~min}$. $[\alpha]_{\mathrm{D}}{ }^{24}=$ $-16.3^{\circ}\left(\mathrm{c} 1.02, \mathrm{CHCl}_{3}\right):{ }^{1} \mathrm{H}$ NMR $\left(400 \mathrm{MHz}, \mathrm{CDCl}_{3}\right) \delta 7.84-7.75(\mathrm{~m}, 2 \mathrm{H}), 7.73(\mathrm{~d}, J=1.9 \mathrm{~Hz}$, $1 \mathrm{H}), 7.67$ (d, $J=8.6 \mathrm{~Hz}, 1 \mathrm{H}), 7.45$ (dtd, $J=14.9,7.5,7.0,5.4 \mathrm{~Hz}, 3 \mathrm{H}), 7.15(\mathrm{dd}, J=8.6,1.9 \mathrm{~Hz}$, $1 \mathrm{H}), 7.11-7.06(\mathrm{~m}, 1 \mathrm{H}), 6.91-6.85(\mathrm{~m}, 1 \mathrm{H}), 2.46-2.30(\mathrm{~m}, 4 \mathrm{H}), 2.27-2.14(\mathrm{~m}, 1 \mathrm{H}), 1.71(\mathrm{~s}$, $3 \mathrm{H}), 1.68(\mathrm{~s}, 3 \mathrm{H}), 0.71(\mathrm{t}, J=7.3 \mathrm{~Hz}, 3 \mathrm{H}) ;{ }^{13} \mathrm{C} \mathrm{NMR}\left(101 \mathrm{MHz}, \mathrm{CDCl}_{3}\right) \delta 148.2,142.8,137.2$, $135.7,133.5,133.4,131.7,128.0,127.7,127.6,127.5,126.3,126.1,125.8,125.3,124.2,46.9$, 32.8, 28.0, 21.9, 20.9, 9.5; FTIR (NaCl/thin film) 3054, 2969, 2934, 2876 1630, 1598, 1502, 1455, 1376, 1265, 1130, 1040, 948, 894, 769, $476 \mathrm{~cm}^{-1}$; HRMS (EI+) [M]+ calculated for $\mathrm{C}_{22} \mathrm{H}_{24}$ : 288.1878, found: 288.1896 .

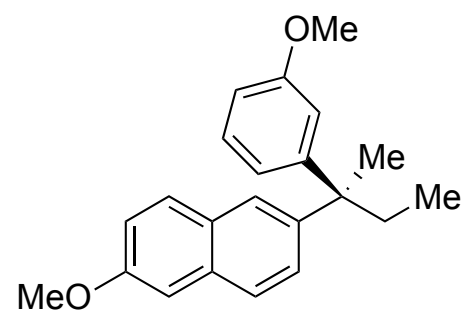

(S)-2-Methoxy-6-(2-(3-methoxyphenyl)butan-2-yl)naphthalene (16). Prepared via General Procedure A using 1b (prepared in 92\% ee) as a colorless sticky oil (run 1: $120 \mathrm{mg}, 94 \%$; run 2: 
$124.9 \mathrm{mg}, 98 \%$ ). The enantiomeric excess was determined to be $90 \%$ (run 1: $90 \%$ ee; run 2: $89 \%$ ee) by chiral HPLC analysis (CHIRALPAK IB, $0.6 \mathrm{~mL} / \mathrm{min}, 0.1 \% i$-PrOH/hexane, $\lambda=254 \mathrm{~nm}$ ); $\mathrm{t}_{\mathrm{R}}($ major $)=16.688 \mathrm{~min}, \mathrm{t}_{\mathrm{R}}($ minor $)=18.948 \mathrm{~min} .[\alpha]_{\mathrm{D}}{ }^{24}=+7.7^{\circ}\left(\mathrm{c} 4.28, \mathrm{CHCl}_{3}\right):{ }^{1} \mathrm{H} \mathrm{NMR}(600$ $\left.\mathrm{MHz}, \mathrm{CDCl}_{3}\right) \delta 7.75-7.69(\mathrm{~m}, 2 \mathrm{H}), 7.59(\mathrm{~d}, J=8.6 \mathrm{~Hz}, 1 \mathrm{H}), 7.21-7.12(\mathrm{~m}, 3 \mathrm{H}), 7.10(\mathrm{~d}, J=$ $2.5 \mathrm{~Hz}, 1 \mathrm{H}), 6.84-6.79(\mathrm{~m}, 2 \mathrm{H}), 6.75-6.70(\mathrm{~m}, 1 \mathrm{H}), 3.91(\mathrm{~s}, 3 \mathrm{H}), 3.75(\mathrm{~s}, 3 \mathrm{H}), 2.30-2.18(\mathrm{~m}$, $2 \mathrm{H}), 1.68(\mathrm{~s}, 3 \mathrm{H}), 0.77(\mathrm{t}, J=7.4 \mathrm{~Hz}, 3 \mathrm{H}) ;{ }^{13} \mathrm{C} \mathrm{NMR}\left(151 \mathrm{MHz}, \mathrm{CDCl}_{3}\right) \delta 159.4,157.6,151.7$, $144.6,132.9,129.6,128.9,128.7,127.7,126.6$, 124.8, 120.4, 118.6, 114.3, 110.2, 105.7, 55.5, 55.2, 46.7, 33.9, 26.9, 9.4; FTIR (NaCl/thin film) 3057, 2967, 2936, 2834, 1609, 1488, 1456, $1388,1264,1198,1032,852,779 \mathrm{~cm}^{-1}$; HRMS $(\mathrm{CI}+)[\mathrm{M}+\mathrm{H}]^{+}$calculated for $\mathrm{C}_{22} \mathrm{H}_{25} \mathrm{O}: 321.1855$, found: 321.1859 .

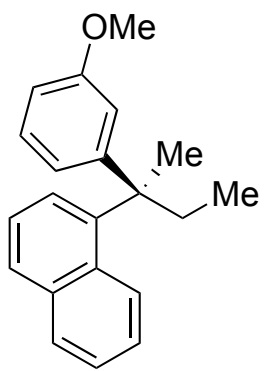

(R)-1-(2-(3-Methoxyphenyl)butan-2-yl)naphthalene (17). Prepared via General Procedure A using 1c (prepared in $90 \%$ ee), except on a $0.30 \mathrm{mmol}$ scale. Product $\mathbf{1 7}$ was isolated as a colorless sticky oil (58.6 mg, 67\%). The enantiomeric excess was determined to be $88 \%$ by chiral HPLC analysis (CHIRALPAK IB, $0.6 \mathrm{~mL} / \mathrm{min}, 0.1 \% \quad i$-PrOH/hexane, $\lambda=254 \mathrm{~nm}$ ); $\mathrm{t}_{\mathrm{R}}$ (major) $=11.227 \mathrm{~min}, \mathrm{t}_{\mathrm{R}}($ minor $)=14.389 \mathrm{~min}$. A duplicate run was performed via General Procedure A using 1c (prepared in $84 \%$ ee), except on a $0.3 \mathrm{mmol} \mathrm{scale}$, to give 17 as a colorless oil $(70.1 \mathrm{mg}, 80 \%)$ in $83 \%$ ee. $[\alpha]_{\mathrm{D}}{ }^{24}=+17.1^{\circ}\left(\mathrm{c} 3.09, \mathrm{CHCl}_{3}\right):{ }^{1} \mathrm{H}$ NMR $\left(600 \mathrm{MHz}, \mathrm{CDCl}_{3}\right) \delta$ $7.81-7.77(\mathrm{~m}, 1 \mathrm{H}), 7.75(\mathrm{~d}, J=8.1 \mathrm{~Hz}, 1 \mathrm{H}), 7.66(\mathrm{dd}, J=7.3,1.2 \mathrm{~Hz}, 1 \mathrm{H}), 7.56(\mathrm{~d}, J=8.8 \mathrm{~Hz}$, 1H), 7.48 (t, $J=7.7 \mathrm{~Hz}, 1 \mathrm{H}), 7.30$ (ddd, $J=8.0,6.7,1.1 \mathrm{~Hz}, 1 \mathrm{H}), 7.16-7.08$ (m, 2H), $6.84-$ $6.80(\mathrm{~m}, 1 \mathrm{H}), 6.80-6.75(\mathrm{~m}, 1 \mathrm{H}), 6.71-6.66(\mathrm{~m}, 1 \mathrm{H}), 3.71(\mathrm{~s}, 3 \mathrm{H}), 2.52(\mathrm{dq}, J=14.6,7.4 \mathrm{~Hz}$, $1 \mathrm{H}), 2.25(\mathrm{dq}, J=13.0,7.3 \mathrm{~Hz}, 1 \mathrm{H}), 1.73(\mathrm{~s}, 3 \mathrm{H}), 0.58(\mathrm{t}, J=7.3 \mathrm{~Hz}, 3 \mathrm{H}) ;{ }^{13} \mathrm{C} \mathrm{NMR}(151 \mathrm{MHz}$, $\left.\mathrm{CDCl}_{3}\right) \delta 159.7,153.1,143.3,135.0,131.6,129.3,129.0,127.9,127.3,125.3,124.9,124.8$, 124.7, 119.4, 113.1, 110.0, 55.2, 47.5, 33.6, 29.4, 9.4; FTIR (NaCl/thin film) 3048, 2969, 2936, 2833, 1604, 1580, 1485, 1289, 1043, 877, 777, $705 \mathrm{~cm}^{-1}$; HRMS (CI+) $[\mathrm{M}+\mathrm{H}]^{+}$calculated for $\mathrm{C}_{21} \mathrm{H}_{23} \mathrm{O}: 291.1749$, found: 291.1747 .

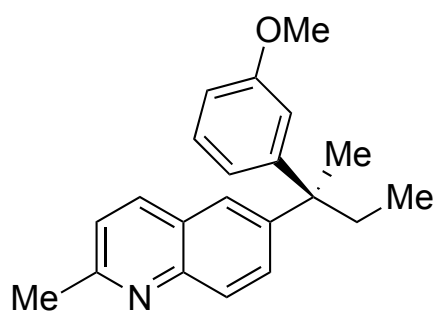

(S)-6-(2-(3-Methoxyphenyl)butan-2-yl)-2-methylquinoline ((S)-18). Prepared via General Procedure A using 1d (prepared in $99 \%$ ee), except that 2a (133 $\mathrm{mg}, 0.332 \mathrm{mmol}, 0.83 \mathrm{equiv}$ ) was used in place of $\mathbf{3 a}$ and the reaction mixture was heated at $60{ }^{\circ} \mathrm{C}$. Product 18 was isolated as a pale yellow oil (run 1: $91 \mathrm{mg}, 74 \%$; run 2: $102.9 \mathrm{mg}, 84 \%$ ). The enantiomeric excess was determined to be $97 \%$ (run 1: $97 \%$ ee; run 2: 97\% ee) by chiral HPLC analysis (CHIRALPAK IA, $1.0 \mathrm{~mL} / \mathrm{min}, 5 \% i-\mathrm{PrOH} / \mathrm{hexane}, \lambda=254 \mathrm{~nm}) ; \mathrm{t}_{\mathrm{R}}$ (major) $=8.758 \mathrm{~min}, \mathrm{t}_{\mathrm{R}}($ minor $)=9.969 \mathrm{~min}$. $[\alpha]_{\mathrm{D}}{ }^{24}=+5.7^{\circ}\left(\mathrm{c} 3.68, \mathrm{CHCl}_{3}\right):{ }^{1} \mathrm{H} \mathrm{NMR}\left(600 \mathrm{MHz}, \mathrm{CDCl}_{3}\right) \delta 8.01(\mathrm{~d}, J=8.4 \mathrm{~Hz}, 1 \mathrm{H}), 7.86(\mathrm{~d}, J$ 
$=8.8 \mathrm{~Hz}, 1 \mathrm{H}), 7.68(\mathrm{~d}, J=2.1 \mathrm{~Hz}, 1 \mathrm{H}), 7.40(\mathrm{dd}, J=8.8,2.2 \mathrm{~Hz}, 1 \mathrm{H}), 7.29-7.24(\mathrm{~m}, 1 \mathrm{H}), 7.19$ $(\mathrm{t}, J=7.9 \mathrm{~Hz}, 1 \mathrm{H}), 6.82-6.76(\mathrm{~m}, 2 \mathrm{H}), 6.75-6.69(\mathrm{~m}, 1 \mathrm{H}), 3.74(\mathrm{~s}, 3 \mathrm{H}), 2.73(\mathrm{~s}, 3 \mathrm{H}), 2.30-$ $2.16(\mathrm{~m}, 2 \mathrm{H}), 1.68(\mathrm{~s}, 3 \mathrm{H}), 0.76(\mathrm{t}, J=7.3 \mathrm{~Hz}, 3 \mathrm{H}) ;{ }^{13} \mathrm{C} \mathrm{NMR}\left(151 \mathrm{MHz}, \mathrm{CDCl}_{3}\right) \delta 159.5,158.6$, $151.1,146.9,146.6,136.4,130.7,129.0,128.3,126.1,124.5,122.0,120.3,114.3,110.4,55.2$, 46.8, 33.9, 26.9, 25.4, 9.3; FTIR (NaCl/thin film) 3053, 2968, 2936, 2833, 1599, 1488, 1431, 1291, 1254, 1173, 1052, 837, $703 \mathrm{~cm}^{-1}$; HRMS (LIFDI) [M]+ calculated for $\mathrm{C}_{21} \mathrm{H}_{23} \mathrm{NO}$ : 305.1780, found: 305.1759 .

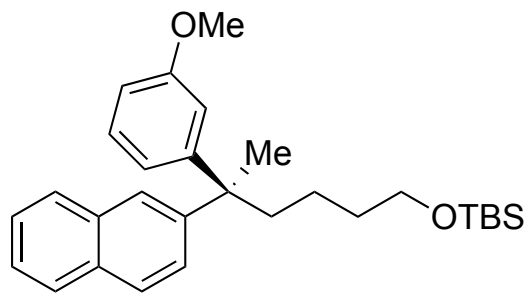

(S)-tert-Butyl((5-(3-methoxyphenyl)-5-(naphthalen-2-yl)hexyl)oxy)dimethylsilane ((S)-19). Prepared via General Procedure A using 1e (prepared in 99\% ee) as a colorless sticky oil (159.8 $\mathrm{mg}, 89 \%$ ). The enantiomeric excess was determined to be $99 \%$ by chiral HPLC analysis (CHIRALPAK IB, $0.4 \mathrm{~mL} / \mathrm{min}, 100 \%$ hexane, $\lambda=254 \mathrm{~nm}$ ); $\mathrm{t}_{\mathrm{R}}$ (major) $=33.672 \mathrm{~min}, \mathrm{t}_{\mathrm{R}}($ minor $)=$ $26.337 \mathrm{~min} .[\alpha]_{\mathrm{D}}{ }^{24}=-11.0^{\circ}\left(\mathrm{c} 5.22, \mathrm{CHCl}_{3}\right):{ }^{1} \mathrm{H} \mathrm{NMR}\left(600 \mathrm{MHz}, \mathrm{CDCl}_{3}\right) \delta 7.82(\mathrm{~d}, J=8.0 \mathrm{~Hz}$, $1 \mathrm{H}), 7.80-7.75(\mathrm{~m}, 2 \mathrm{H}), 7.68(\mathrm{~d}, J=8.6 \mathrm{~Hz}, 1 \mathrm{H}), 7.45$ (dddd, $J=19.3,8.1,6.8,1.4 \mathrm{~Hz}, 2 \mathrm{H})$, $7.21-7.15(\mathrm{~m}, 2 \mathrm{H}), 6.83-6.78(\mathrm{~m}, 2 \mathrm{H}), 6.75-6.70(\mathrm{~m}, 1 \mathrm{H}), 3.75(\mathrm{~s}, 3 \mathrm{H}), 3.55(\mathrm{t}, J=6.6 \mathrm{~Hz}$, $2 \mathrm{H}), 2.26-2.15(\mathrm{~m}, 2 \mathrm{H}), 1.72(\mathrm{~s}, 3 \mathrm{H}), 1.56-1.49(\mathrm{~m}, 2 \mathrm{H}), 1.22-1.09(\mathrm{~m}, 2 \mathrm{H}), 0.84(\mathrm{~s}, 9 \mathrm{H}),-$ $0.01(\mathrm{~s}, 6 \mathrm{H}) ;{ }^{13} \mathrm{C}$ NMR $\left(151 \mathrm{MHz}, \mathrm{CDCl}_{3}\right) \delta 159.4,151.6,147.0,133.3,131.9,129.0,128.1$, 127.7, 127.5, 127.1, 125.9, 125.5, 124.7, 120.3, 114.2, 110.3, 63.2, 55.2, 46.6, 41.4, 33.7, 27.6, 26.1, 21.3, 18.4, -5.1; FTIR (NaCl/thin film) 3055, 2934, 2856, 1606, 1470, 1255, 1099, 1046, 836, 775, 705, $476 \mathrm{~cm}^{-1}$; HRMS (LIFDI) [M]+ calculated for $\mathrm{C}_{29} \mathrm{H}_{40} \mathrm{O}_{2} \mathrm{Si}$ : 448.2798, found: 448.2790 .

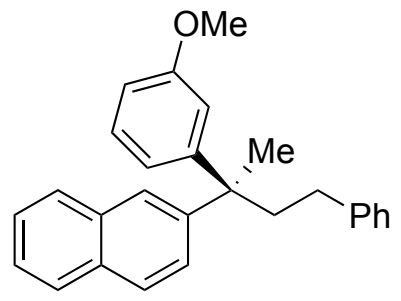

(S)-2-(2-(3-Methoxyphenyl)-4-phenylbutan-2-yl)naphthalene ((S)-20). Prepared via General Procedure A using 1f (prepared in 94\% ee), except that the reaction was run for $48 \mathrm{~h}$. Product 20 was obtained as a colorless oil (run 1: $171.5 \mathrm{mg}, 90 \%$; run 2: $137.8 \mathrm{mg}$, 94\%). The enantiomeric excess was determined to be $94 \%$ (run1: $94 \%$ ee; run 2: $94 \%$ ee) by chiral HPLC analysis $($ CHIRALPAK IB, $0.8 \mathrm{~mL} / \mathrm{min}, 100 \%$ hexane, $\lambda=210 \mathrm{~nm}) ; \mathrm{t}_{\mathrm{R}}$ (major) $=34.901 \mathrm{~min}, \mathrm{t}_{\mathrm{R}}($ minor $)=$ $31.785 \mathrm{~min} .[\alpha]_{\mathrm{D}}{ }^{24}=-25.9^{\circ}\left(\mathrm{c} 4.54, \mathrm{CHCl}_{3}\right):{ }^{1} \mathrm{H} \mathrm{NMR}\left(600 \mathrm{MHz}, \mathrm{CDCl}_{3}\right) \delta 7.87-7.83(\mathrm{~m}, 2 \mathrm{H})$, $7.81(\mathrm{dd}, J=7.8,1.5 \mathrm{~Hz}, 1 \mathrm{H}), 7.73(\mathrm{~d}, J=8.7 \mathrm{~Hz}, 1 \mathrm{H}), 7.52-7.44(\mathrm{~m}, 2 \mathrm{H}), 7.29$ (t, $J=7.6 \mathrm{~Hz}$, $2 \mathrm{H}), 7.25-7.17(\mathrm{~m}, 3 \mathrm{H}), 7.17-7.13(\mathrm{~m}, 2 \mathrm{H}), 6.88-6.85(\mathrm{~m}, 2 \mathrm{H}), 6.76(\mathrm{ddd}, J=8.3,2.4,1.0$ $\mathrm{Hz}, 1 \mathrm{H}), 3.76(\mathrm{~s}, 3 \mathrm{H}), 2.59-2.49(\mathrm{~m}, 2 \mathrm{H}), 2.49-2.37(\mathrm{~m}, 2 \mathrm{H}), 1.84(\mathrm{~s}, 3 \mathrm{H}) ;{ }^{13} \mathrm{C}$ NMR $(151$ $\left.\mathrm{MHz}_{,} \mathrm{CDCl}_{3}\right) \delta 159.6,151.1,146.6,143.0,133.3,132.0,129.1,128.51,128.50,128.2,127.9$, 127.5, 127.0, 126.1, 125.9, 125.7, 124.8, 120.2, 114.2, 110.5, 55.3, 46.7, 43.9, 31.5, 27.5; FTIR (NaCl/thin film) 3056, 3024, 2946, 2867, 1600, 1283, 1494, 1291, 1047, 908, 818, $760 \mathrm{~cm}^{-1}$; HRMS (EI+) [M]+ calculated for $\mathrm{C}_{27} \mathrm{H}_{26} \mathrm{O}: 366.1984$, found: 366.1967 . 
Please note: The absolute configuration of $\mathbf{2 0}$ is tentatively assigned. The absolute configuration resulting from the method used in the preparation of its alcohol precursor S-1f has not been reported in the literature. Please see the experimental for $\mathbf{S - 1 f}$ below.

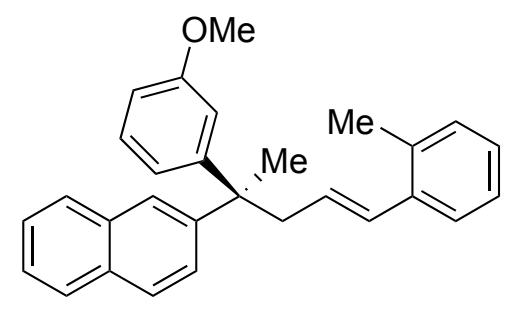

(E)-2-(2-(3-Methoxyphenyl)-5-(o-tolyl)pent-4-en-2-yl)naphthalene (21). Prepared via General Procedure A using 1g (prepared in $96 \%$ ee), except that 2a (133 mg, $0.332 \mathrm{mmol}, 0.83$ equiv) was used in place of 3a. Product 21 was isolated as a colorless sticky oil (run 1: $125.6 \mathrm{mg}, 80 \%$; run 2: $119.7 \mathrm{mg}, 76 \%$ ). The enantiomeric excess was determined to be $95 \%$ (run 1: $95 \%$ ee; run 2: $94 \%$ ee) by chiral HPLC analysis (CHIRALPAK IC, $0.6 \mathrm{~mL} / \mathrm{min}, 0.1 \%$ hexane, $\lambda=210 \mathrm{~nm}$ ); $\mathrm{t}_{\mathrm{R}}($ major $)=13.480 \mathrm{~min}, \mathrm{t}_{\mathrm{R}}($ minor $)=15.096 \mathrm{~min} .[\alpha]_{\mathrm{D}}{ }^{24}=+3.7^{\circ}\left(\mathrm{c} 4.84, \mathrm{CHCl}_{3}\right):{ }^{1} \mathrm{H}$ NMR $(600$ $\left.\mathrm{MHz}, \mathrm{CDCl}_{3}\right) \delta 7.85-7.81(\mathrm{~m}, 2 \mathrm{H}), 7.81-7.76(\mathrm{~m}, 1 \mathrm{H}), 7.71(\mathrm{~d}, J=8.6 \mathrm{~Hz}, 1 \mathrm{H}), 7.50-7.42$ $(\mathrm{m}, 2 \mathrm{H}), 7.24(\mathrm{dd}, J=8.7,2.0 \mathrm{~Hz}, 1 \mathrm{H}), 7.20(\mathrm{t}, J=7.9 \mathrm{~Hz}, 1 \mathrm{H}), 7.17(\mathrm{~d}, J=7.3 \mathrm{~Hz}, 1 \mathrm{H}), 7.10-$ $7.01(\mathrm{~m}, 3 \mathrm{H}), 6.88-6.82(\mathrm{~m}, 2 \mathrm{H}), 6.77-6.72(\mathrm{~m}, 1 \mathrm{H}), 6.57(\mathrm{~d}, J=15.6 \mathrm{~Hz}, 1 \mathrm{H}), 5.76(\mathrm{dt}, J=$ 15.6, 7.2 Hz, 1H), $3.74(\mathrm{~s}, 3 \mathrm{H}), 3.21-3.10(\mathrm{~m}, 2 \mathrm{H}), 2.20(\mathrm{~s}, 3 \mathrm{H}), 1.76(\mathrm{~s}, 3 \mathrm{H}) ;{ }^{13} \mathrm{C} \mathrm{NMR}(151$ $\left.\mathrm{MHz}, \mathrm{CDCl}_{3}\right) \delta 159.5,151.0,146.5,137.1,135.1,133.3,132.0,131.1,130.1,129.1,128.5$, $128.2,127.8,127.5,127.1,127.0,126.04,126.02,125.9,125.7,124.8,120.3,114.2,110.6,55.3$, 46.8, 45.6, 27.7, 19.8; FTIR (NaCl/thin film) 3054, 2965, 2933, 1599, 1485, 1431, 1258, 1047 , $967,818,754 \mathrm{~cm}^{-1}$; HRMS (LIFDI) [M]+ calculated for $\mathrm{C}_{29} \mathrm{H}_{28} \mathrm{O}: 392.2140$, found: 392.2137 .

Please note: The absolute configuration of $\mathbf{2 1}$ is tentatively assigned. The absolute configuration resulting from the method used in the preparation of its acetate precursor $\mathbf{1 g}$ has not been reported in the literature. Please see the experimental for $\mathbf{1 g}$ below.<smiles>CCC(CC)(CCc1cccc(OC)c1)c1ccc2ccccc2c1</smiles>

(S)-2-(1,3-Bis(3-methoxyphenyl)pentan-3-yl)naphthalene ((S)-22). Prepared via General Procedure A using $\mathbf{1 h}$ (prepared in $89 \%$ ee), except that $\mathbf{2 a}$ (133 $\mathrm{mg}, 0.332 \mathrm{mmol}, 0.83$ equiv) was used in place of 3a. Product 22 was isolated as a colorless sticky oil (127 mg, 77\%). The enantiomeric excess was determined to be $87 \%$ by chiral SFC analysis (CHIRALCEL OJ-H(25 x $0.46 \mathrm{~cm}), 3.0 \mathrm{~mL} / \mathrm{min}, 20 \% \mathrm{MeOH}(0.1 \%$ diethylamine $\left.) / \mathrm{CO}_{2}(100 \mathrm{bar}), \lambda=220 \mathrm{~nm}\right) ; \mathrm{t}_{\mathrm{R}}($ major $)=$ $9.10 \mathrm{~min}, \mathrm{t}_{\mathrm{R}}$ (minor) $=7.81 \mathrm{~min}$. A duplicate experiment was conducted with $\mathbf{1 h}$ (prepared in $87 \%$ ee) to give $22(103 \mathrm{mg}, 63 \%)$ in $86 \%$ ee. $[\alpha]_{\mathrm{D}}{ }^{24}=-30.5^{\circ}$ (c 3.04, $\left.\mathrm{CHCl}_{3}\right):{ }^{1} \mathrm{H}$ NMR $(600$ $\left.\mathrm{MHz}, \mathrm{CDCl}_{3}\right) \delta 7.87-7.81(\mathrm{~m}, 2 \mathrm{H}), 7.78(\mathrm{~d}, J=7.8 \mathrm{~Hz}, 1 \mathrm{H}), 7.68(\mathrm{~d}, J=8.7 \mathrm{~Hz}, 1 \mathrm{H}), 7.51-$ $7.41(\mathrm{~m}, 2 \mathrm{H}), 7.19(\mathrm{td}, J=7.9,4.1 \mathrm{~Hz}, 2 \mathrm{H}), 7.14(\mathrm{dd}, J=8.7,1.9 \mathrm{~Hz}, 1 \mathrm{H}), 6.86-6.81(\mathrm{~m}, 2 \mathrm{H})$, $6.77-6.67(\mathrm{~m}, 3 \mathrm{H}), 6.65-6.61(\mathrm{~m}, 1 \mathrm{H}), 3.78(\mathrm{~s}, 3 \mathrm{H}), 3.75(\mathrm{~s}, 3 \mathrm{H}), 2.50-2.44(\mathrm{~m}, 2 \mathrm{H}), 2.36-$ $2.22(\mathrm{~m}, 4 \mathrm{H}), 0.75(\mathrm{t}, J=7.3 \mathrm{~Hz}, 3 \mathrm{H}) ;{ }^{13} \mathrm{C} \mathrm{NMR}\left(151 \mathrm{MHz}, \mathrm{CDCl}_{3}\right) \delta 159.8,159.4,150.1$, $145.7,144.8,133.2,131.9,129.5,128.9,128.2,127.7,127.51,127.48,126.0,125.6,125.5$, $120.93,120.92,114.9,114.4,111.0,110.4,55.29,55.28,50.0,38.9,30.9,29.5$, 8.6; FTIR 
(NaCl/thin film) 3054, 2955, 2833, 1600, 1487, 1257, 1153, 1050, 908, 813, $782 \mathrm{~cm}^{-1}$; HRMS (LIFDI) [M]+ calculated for $\mathrm{C}_{29} \mathrm{H}_{30} \mathrm{O}_{2}: 410.2246$, found: 410.2238 .

Please note: The absolute configuration of $\mathbf{2 2}$ is tentatively assigned. The absolute configuration resulting from the method used in the preparation of its alcohol precursor $\mathbf{S}-\mathbf{1} \mathbf{h}$ has not been reported in the literature. Please see the experimental for $\mathbf{S}-\mathbf{1 h}$ below.

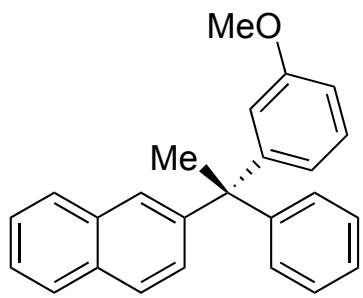

(R)-2-(1-(3-Methoxyphenyl)-1-phenylethyl)naphthalene $((\boldsymbol{R})-23)$. Prepared via General Procedure A using 1i (prepared in 96\% ee) as a colorless oil (run 1: $95 \mathrm{mg}, 70 \%$; run 2: $101 \mathrm{mg}$, $75 \%$ ). The enantiomeric excess was determined to be $94 \%$ (run 1: $94 \%$; run 2: $94 \%$ ) by chiral HPLC analysis (CHIRALPAK IB, $0.2 \mathrm{~mL} / \mathrm{min}, 0.1 \%$ hexane, $\lambda=210 \mathrm{~nm}$ ); $\mathrm{t}_{\mathrm{R}}$ (major) $=49.084$ min, $\mathrm{t}_{\mathrm{R}}$ (minor $)=52.102 \mathrm{~min} .[\alpha]_{\mathrm{D}}{ }^{24}=+15.0^{\circ}\left(\mathrm{c} 0.86, \mathrm{CHCl}_{3}\right):{ }^{1} \mathrm{H}$ NMR $\left(600 \mathrm{MHz}, \mathrm{CDCl}_{3}\right) \delta$ $7.82-7.79(\mathrm{~m}, 1 \mathrm{H}), 7.74(\mathrm{~d}, J=8.7 \mathrm{~Hz}, 1 \mathrm{H}), 7.72-7.67(\mathrm{~m}, 1 \mathrm{H}), 7.49-7.41(\mathrm{~m}, 3 \mathrm{H}), 7.32(\mathrm{dd}$, $J=8.6,2.0 \mathrm{~Hz}, 1 \mathrm{H}), 7.28(\mathrm{t}, J=7.4 \mathrm{~Hz}, 2 \mathrm{H}), 7.25-7.19(\mathrm{~m}, 2 \mathrm{H}), 7.19-7.14(\mathrm{~m}, 2 \mathrm{H}), 6.81-$ $6.74(\mathrm{~m}, 2 \mathrm{H}), 6.72(\mathrm{t}, J=2.1 \mathrm{~Hz}, 1 \mathrm{H}), 3.72(\mathrm{~s}, 3 \mathrm{H}), 2.27(\mathrm{~s}, 3 \mathrm{H}) ;{ }^{13} \mathrm{C} \mathrm{NMR}\left(151 \mathrm{MHz}, \mathrm{CDCl}_{3}\right) \delta$ $159.4,150.6,148.8,146.5,133.2,132.0,130.6,128.94,128.91,128.3,128.1,127.8,127.5$, 127.0, 126.2, 126.0, 125.9, 121.7, 115.6, 110.9, 55.3, 52.9, 30.6; FTIR (NaCl/thin film) 3055, 2978, 2934, 2833, 1597, 1487, 1256, 1044, 820, 745, $701 \mathrm{~cm}^{-1}$; HRMS (CI+) $[\mathrm{M}+\mathrm{H}]^{+}$calculated for $\mathrm{C}_{25} \mathrm{H}_{23} \mathrm{O}: 339.1749$, found: 339.1742 .<smiles>COc1cccc(C(C)(c2ccccc2)c2ccc(-c3ccccc3)cc2)c1</smiles>

(R)-4-(1-(3-Methoxyphenyl)-1-phenylethyl)-1,1'-biphenyl ((R)-24). Prepared via General Procedure A using 1j (prepared as 91\% ee), except with $10 \mathrm{~mol} \% \mathrm{NiCl}_{2} \cdot \mathrm{DME}, 10 \mathrm{~mol} \%$ CyJohnPhos, $60{ }^{\circ} \mathrm{C}, 48 \mathrm{~h}$. Product 24 was isolated as a colorless oil (run 1: $84.4 \mathrm{mg}, 58 \%$; run 2: $96.2 \mathrm{mg}, 66 \%$ ). The enantiomeric excess was determined to be $91 \%$ (run 1: $91 \%$ ee; run $2: 91 \%$ ee) by chiral HPLC analysis (CHIRALPAK IB, $0.6 \mathrm{~mL} / \mathrm{min}, 0.1 \%$ hexane, $\lambda=254 \mathrm{~nm}$ ); $\mathrm{t}_{\mathrm{R}}$ (major) $=18.913 \mathrm{~min}, \mathrm{t}_{\mathrm{R}}($ minor $)=18.288 \mathrm{~min} .[\alpha]_{\mathrm{D}}{ }^{24}=+31.5^{\circ}\left(\mathrm{c} 1.68, \mathrm{CHCl}_{3}\right):{ }^{1} \mathrm{H} \mathrm{NMR}(600 \mathrm{MHz}$, $\left.\mathrm{CDCl}_{3}\right) \delta 7.60(\mathrm{dd}, J=8.1,1.4 \mathrm{~Hz}, 2 \mathrm{H}), 7.54-7.48(\mathrm{~m}, 2 \mathrm{H}), 7.43(\mathrm{t}, J=7.7 \mathrm{~Hz}, 2 \mathrm{H}), 7.35-$ $7.31(\mathrm{~m}, 1 \mathrm{H}), 7.29(\mathrm{dd}, J=8.4,6.9 \mathrm{~Hz}, 2 \mathrm{H}), 7.22(\mathrm{td}, J=7.6,4.0 \mathrm{~Hz}, 2 \mathrm{H}), 7.20-7.14(\mathrm{~m}, 4 \mathrm{H})$, $6.80-6.73(\mathrm{~m}, 2 \mathrm{H}), 6.71(\mathrm{t}, J=2.1 \mathrm{~Hz}, 1 \mathrm{H}), 3.74(\mathrm{~s}, 3 \mathrm{H}), 2.22(\mathrm{~s}, 3 \mathrm{H}) ;{ }^{13} \mathrm{C} \mathrm{NMR}(151 \mathrm{MHz}$, $\left.\mathrm{CDCl}_{3}\right) \delta 159.4,150.8,149.0,148.2,140.9,138.8,129.3,128.90,128.86,128.85,128.0,127.3$, 127.1, 126.6, 126.2, 121.6, 115.6, 110.8, 55.3, 52.5, 30.6; FTIR (NaCl/thin film) 3055, 3028, $2979,2833,1598,1486,1290,1254,1040,845,735,699 \mathrm{~cm}^{-1}$; HRMS $(\mathrm{CI}+)[\mathrm{M}+\mathrm{H}]^{+}$calculated for $\mathrm{C}_{27} \mathrm{H}_{25} \mathrm{O}: 365.1905$, found: 365.1907 . 


\title{
Preparation of Enantioenriched Tertiary Benzyl Acetates
}

\author{
General Procedure B: Preparation of (S)-2-(Naphthalen-2-yl)butan-2-yl Acetate (1a).

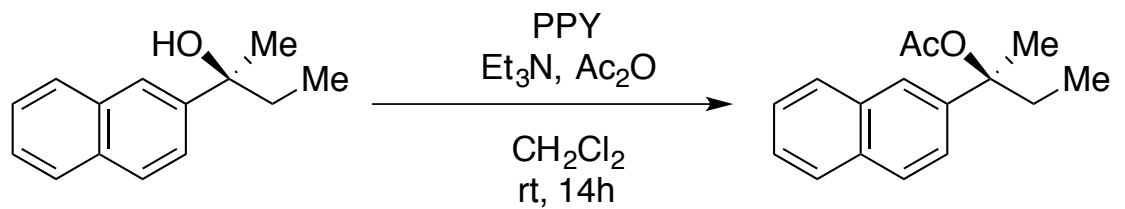

In an oven-dried 100-mL round-bottomed flask, was placed 2-(naphthalen-2-yl)butan-2ol (S-1a, prepared in 96\% ee, $1.5 \mathrm{~g}, 7.5 \mathrm{mmol}, 1.0$ equiv), 4-pyrrolidinopyridine (PPY, $168 \mathrm{mg}$, 1.13 mmol, 0.150 equiv), and $\mathrm{CH}_{2} \mathrm{Cl}_{2}(25 \mathrm{~mL}, 0.3 \mathrm{M})$. Then flask was then placed in an ice/water bath. $\mathrm{Et}_{3} \mathrm{~N}$ (3.1 mL, $23 \mathrm{mmol}, 3.0$ equiv) was added, followed by acetic anhydride (1.4 $\mathrm{mL}, 15 \mathrm{mmol}, 2.0$ equiv). The solution was then stirred at room temperature for $14 \mathrm{~h}$. Sat. $\mathrm{NaHCO}_{3}(100 \mathrm{~mL})$ was added, and the product was extracted with $\mathrm{CH}_{2} \mathrm{Cl}_{2}(3 \times 25 \mathrm{~mL})$. The combined organic layers were washed with sat. $\mathrm{NaCl}$, dried $\left(\mathrm{Na}_{2} \mathrm{SO}_{4}\right)$, filtered and concentrated. The crude mixture was purified by silica gel chromatography (0-20\% EtOAc/hexanes) to give $1 \mathrm{a}$ as a viscous oil (1.45 g, 80\%). The enantiomeric excess was determined to be $96 \%$ by chiral HPLC analysis (CHIRALPAK IB, $1 \mathrm{~mL} / \mathrm{min}, 1 \% i-\mathrm{PrOH} /$ hexane, $\lambda=254 \mathrm{~nm}$ ); $\mathrm{t}_{\mathrm{R}}$ (major) $=8.234$ $\min , \mathrm{t}_{\mathrm{R}}$ (minor) $=6.313 \mathrm{~min} .[\alpha]_{\mathrm{D}}{ }^{24}=+5.0^{\circ}\left(\mathrm{c} 3.59, \mathrm{CHCl}_{3}\right):{ }^{1} \mathrm{H}$ NMR $\left(600 \mathrm{MHz}, \mathrm{CDCl}_{3}\right) \delta 7.86$ $-7.78(\mathrm{~m}, 3 \mathrm{H}), 7.77-7.74(\mathrm{~m}, 1 \mathrm{H}), 7.51-7.39(\mathrm{~m}, 3 \mathrm{H}), 2.18-2.08(\mathrm{~m}, 5 \mathrm{H}), 1.92(\mathrm{~s}, 3 \mathrm{H}), 0.81$ $(\mathrm{t}, J=7.4 \mathrm{~Hz}, 3 \mathrm{H}) ;{ }^{13} \mathrm{C} \mathrm{NMR}\left(151 \mathrm{MHz}, \mathrm{CDCl}_{3}\right) \delta 169.8,142.4,133.2,132.5,128.3,128.1$, 127.6, 126.2, 125.9, 123.6, 123.2, 84.6, 35.1, 24.5, 22.4, 8.3; FTIR (NaCl/thin film) 3057, 2977, 2938, 2880, 1734, 1458, 1366, 1246, 1128, 1017, 817, 747, $477 \mathrm{~cm}^{-1}$; HRMS (EI+) [M]+ calculated for $\mathrm{C}_{16} \mathrm{H}_{18} \mathrm{O}_{2}: 242.1307$, found: 242.1309 .

This type of compound decomposed to olefins quickly in pure form at room temperature, but is relatively stable in cold solution. Our suggestion is to immediately dissolve in anhydrous 2-Me-THF and store in fridge under $\mathrm{N}_{2}$.

A crystal suitable for X-ray diffraction analysis was obtained upon cooling the viscous oil isolated above neat at $-35^{\circ} \mathrm{C}$. The crystal structure demonstrates that the absolute configuration is $S$ (Figure $\mathrm{S} 2$ ).

Figure S2. Molecular diagram of $(S)$-1a with ellipsoids at 50\% probability, H-atoms omitted for clarity. (CCDC 1502353)

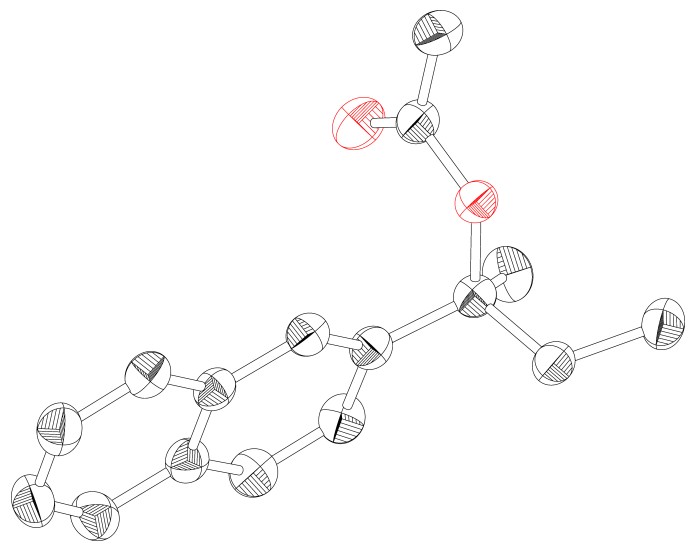




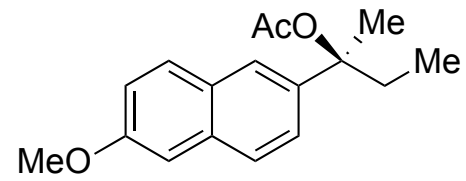

(S)-2-(6-Methoxynaphthalen-2-yl)butan-2-yl acetate ((S)-1b). Prepared via General Procedure B using S-1b (prepared as $92 \%$ ee) as a colorless oil (75\%). The enantiomeric excess was assumed to be $92 \%$ based on the starting material $(\mathbf{S}-\mathbf{1 b}) \cdot[\alpha]_{\mathrm{D}}{ }^{24}=+42^{\circ}\left(\mathrm{c} 1.5, \mathrm{CHCl}_{3}\right):{ }^{1} \mathrm{H} \mathrm{NMR}$ $\left(600 \mathrm{MHz}, \mathrm{CDCl}_{3}\right) \delta 7.74-7.66(\mathrm{~m}, 3 \mathrm{H}), 7.40(\mathrm{dd}, J=8.6,1.9 \mathrm{~Hz}, 1 \mathrm{H}), 7.14(\mathrm{dd}, J=8.8,2.5$ $\mathrm{Hz}, 1 \mathrm{H}), 7.11(\mathrm{~d}, J=2.5 \mathrm{~Hz}, 1 \mathrm{H}), 3.91(\mathrm{~s}, 3 \mathrm{H}), 2.16-2.06(\mathrm{~m}, 5 \mathrm{H}), 1.90(\mathrm{~s}, 3 \mathrm{H}), 0.80(\mathrm{t}, J=7.4$ $\mathrm{Hz}, 3 \mathrm{H}) ;{ }^{13} \mathrm{C}$ NMR $\left(151 \mathrm{MHz}, \mathrm{CDCl}_{3}\right) \delta 169.9,157.8,140.1,133.6,129.8,128.7,126.9,123.8$, 123.5, 119.0, 105.7, 84.7, 55.5, 35.1, 24.5, 22.4, 8.3; FTIR (NaCl/thin film) 2975, 2937, 1734, $1608,1367,1247,1204,1164,1031,850 \mathrm{~cm}^{-1}$; HRMS $(\mathrm{CI}+)[\mathrm{M}]+\mathrm{H}$ calculated for $\mathrm{C}_{17} \mathrm{H}_{21} \mathrm{O}_{3}$ : 273.1491, found: 273.1501 .<smiles>CCC(C)(OC)c1cccc2ccccc12</smiles>

(S)-2-(Naphthalen-1-yl)butan-2-yl acetate ((S)-1c). Prepared via General Procedure B using S1c $(90 \%$ ee) as a colorless oil $(58 \%)$. The enantiomeric excess was determined to be $90 \%$ by chiral HPLC analysis (CHIRALPAK IA, $0.8 \mathrm{~mL} / \mathrm{min}, 1.0 \% i$-PrOH/hexanes, $\lambda=254 \mathrm{~nm}$ ); $\mathrm{t}_{\mathrm{R}}$ (major) $=9.214 \mathrm{~min}, \mathrm{t}_{\mathrm{R}}($ minor $)=8.322 \mathrm{~min} .[\alpha]_{\mathrm{D}}{ }^{24}=+10.2^{\circ}\left(\mathrm{c} 0.88, \mathrm{CHCl}_{3}\right):{ }^{1} \mathrm{H}$ NMR $(600$ $\left.\mathrm{MHz} \mathrm{CDCl}_{3}\right) \delta 8.55(\mathrm{dd}, J=8.5,1.5 \mathrm{~Hz}, 1 \mathrm{H}), 7.87(\mathrm{dd}, J=7.7,1.9 \mathrm{~Hz}, 1 \mathrm{H}), 7.78(\mathrm{~d}, J=8.1 \mathrm{~Hz}$, $1 \mathrm{H}), 7.52(\mathrm{dd}, J=7.3,1.3 \mathrm{~Hz}, 1 \mathrm{H}), 7.50-7.41(\mathrm{~m}, 3 \mathrm{H}), 2.48(\mathrm{dq}, J=14.7,7.5 \mathrm{~Hz}, 1 \mathrm{H}), 2.25$ $(\mathrm{dq}, J=14.6,7.5 \mathrm{~Hz}, 1 \mathrm{H}), 2.04(\mathrm{~s}, 3 \mathrm{H}), 2.02(\mathrm{~s}, 3 \mathrm{H}), 0.87(\mathrm{t}, J=7.5 \mathrm{~Hz}, 3 \mathrm{H}) ;{ }^{13} \mathrm{C}$ NMR $(151$ $\left.\mathrm{MHz} \mathrm{CDCl}_{3}\right) \delta 169.5,139.9,134.9,130.4,129.7,128.7,125.6,125.5,125.1,125.0,124.3,85.6$, 34.0, 24.6, 21.8, 8.7; FTIR (NaCl/thin film) 2979, 2940, 1734, 1653, 1558, 1507, 1364, 1242, 1107, 1015, 804, $776 \mathrm{~cm}^{-1}$; HRMS (EI+) [M]+ calculated for $\mathrm{C}_{16} \mathrm{H}_{18} \mathrm{O}_{2}$ : 242.1307, found: 242.1316.<smiles>CCC(C)(OC)c1ccc2nc(C)ccc2c1</smiles>

(S)-2-(2-Methylquinolin-6-yl)butan-2-yl acetate ((S)-1d). Prepared via General Procedure B using S-1d (99\% ee) as a yellow oil (87\%). The enantiomeric excess was determined to be $99 \%$ by chiral HPLC analysis (CHIRLPAK IB, $1.0 \mathrm{~mL} / \mathrm{min}, 6.0 \% i$-PrOH/hexanes, $\lambda=254 \mathrm{~nm}$ ); $\mathrm{t}_{\mathrm{R}}($ major $)=13.331 \mathrm{~min}, \mathrm{t}_{\mathrm{R}}($ minor $)=9.787 \mathrm{~min} .[\alpha]_{\mathrm{D}}{ }^{24}=+7.8^{\circ}\left(\mathrm{c} 1.51, \mathrm{CHCl}_{3}\right):{ }^{1} \mathrm{H} \mathrm{NMR}(600$ $\left.\mathrm{MHz}, \mathrm{CDCl}_{3}\right) \delta 8.01(\mathrm{~d}, J=8.4 \mathrm{~Hz}, 1 \mathrm{H}), 7.97(\mathrm{~d}, J=8.8 \mathrm{~Hz}, 1 \mathrm{H}), 7.67(\mathrm{~d}, J=2.1 \mathrm{~Hz}, 1 \mathrm{H}), 7.63$ $(\mathrm{dd}, J=8.8,2.2 \mathrm{~Hz}, 1 \mathrm{H}), 7.27(\mathrm{~s}, 1 \mathrm{H}), 2.72(\mathrm{~s}, 3 \mathrm{H}), 2.10(\mathrm{~s}, 5 \mathrm{H}), 1.90(\mathrm{~s}, 3 \mathrm{H}), 0.79(\mathrm{t}, J=7.4 \mathrm{~Hz}$, $3 \mathrm{H}) ;{ }^{13} \mathrm{C}$ NMR $\left(151 \mathrm{MHz}, \mathrm{CDCl}_{3}\right) \delta 169.8,159.0,147.1,142.2,136.5,128.7,126.7,126.1$, 123.2, 122.3, 84.3, 35.1, 25.4, 24.4, 22.3, 8.3; FTIR (NaCl/thin film) 2977, 2938, 1739, 1601, 1368, 1247, 1136, 1078, $834 \mathrm{~cm}^{-1}$; HRMS (CI+) [M]+H calculated for $\mathrm{C}_{16} \mathrm{H}_{20} \mathrm{NO}_{2}$ : 258.1494, found: 258.1488 . 


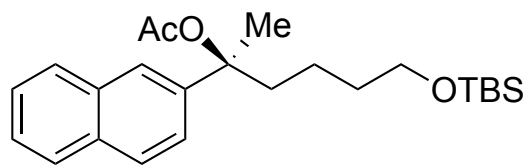

(S)-6-((tert-Butyldimethylsilyl)oxy)-2-(naphthalen-2-yl)hexan-2-yl acetate $((S)-1 e)$. Prepared via General Procedure B using S-1e (99\% ee) as a colorless oil $(75 \%)$. The enantiomeric excess was determined to be $99 \%$ by chiral HPLC analysis (CHIRLCEL OD-H, $1.0 \mathrm{~mL} / \mathrm{min}, 0.5 \% i$ $\mathrm{PrOH} /$ hexanes, $\lambda=254 \mathrm{~nm}) ; \mathrm{t}_{\mathrm{R}}($ major $)=21.535 \mathrm{~min}, \mathrm{t}_{\mathrm{R}}($ minor $)=14.469 \mathrm{~min} .[\alpha]_{\mathrm{D}}{ }^{24}=+15.4^{\circ}(\mathrm{c}$ 4.73, $\left.\mathrm{CHCl}_{3}\right):{ }^{1} \mathrm{H}$ NMR $\left(600 \mathrm{MHz}, \mathrm{CDCl}_{3}\right) \delta 7.81(\mathrm{dd}, J=11.1,8.5 \mathrm{~Hz}, 3 \mathrm{H}), 7.76-7.71(\mathrm{~m}$, $1 \mathrm{H}), 7.48-7.41(\mathrm{~m}, 3 \mathrm{H}), 3.53(\mathrm{t}, J=6.4 \mathrm{~Hz}, 2 \mathrm{H}), 2.14-2.03(\mathrm{~m}, 5 \mathrm{H}), 1.93(\mathrm{~s}, 3 \mathrm{H}), 1.46(\mathrm{p}, J=$ $7.0 \mathrm{~Hz}, 2 \mathrm{H}), 1.31-1.22(\mathrm{~m}, 2 \mathrm{H}), 0.83(\mathrm{~s}, 9 \mathrm{H}),-0.01(\mathrm{~s}, 6 \mathrm{H}) ;{ }^{13} \mathrm{C}$ NMR $\left(101 \mathrm{MHz}, \mathrm{CDCl}_{3}\right) \delta$ $169.9,142.5,133.2,132.5,128.3,128.1,127.6,126.1,125.9,123.5,123.1,84.2,63.0,42.4,33.0$, 26.0, 24.8, 22.4, 20.3, 18.4, -5.2; FTIR (NaCl/thin film) 3058, 2952, 2929, 2857, 1739, 1366, 1248, 1101, 836, $775 \mathrm{~cm}^{-1}$; HRMS (LIFDI) [M]+ calculated for $\mathrm{C}_{24} \mathrm{H}_{36} \mathrm{O}_{3} \mathrm{Si}: 400.2434$, found: 400.2435 .

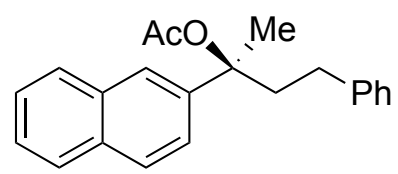

(S)-2-(Naphthalen-2-yl)-4-phenylbutan-2-yl acetate ((S)-1f). Prepared via General Procedure $\mathrm{B}$ as a colorless oil $(96 \%)$. The enantiomeric excess was determine to be $94 \%$ by chiral HPLC analysis (CHIRALPAK IA, $1.0 \mathrm{~mL} / \mathrm{min}, 0.5 \% i$-PrOH/hexanes, $\lambda=254 \mathrm{~nm}$ ); $\mathrm{t}_{\mathrm{R}}$ (major) $=11.227$ $\min , \mathrm{t}_{\mathrm{R}}($ minor $)=12.577 \mathrm{~min} . \quad[\alpha]_{\mathrm{D}}{ }^{24}=-74.8^{\circ}\left(\mathrm{c} 1.1, \mathrm{CHCl}_{3}\right):{ }^{1} \mathrm{H}$ NMR $\left(400 \mathrm{MHz}, \mathrm{CDCl}_{3}\right) \delta$ $7.89-7.77(\mathrm{~m}, 4 \mathrm{H}), 7.52-7.43(\mathrm{~m}, 3 \mathrm{H}), 7.26-7.21(\mathrm{~m}, 2 \mathrm{H}), 7.16(\mathrm{~d}, J=7.3 \mathrm{~Hz}, 1 \mathrm{H}), 7.13-$ $7.07(\mathrm{~m}, 2 \mathrm{H}), 2.58-2.34(\mathrm{~m}, 4 \mathrm{H}), 2.12(\mathrm{~s}, 3 \mathrm{H}), 2.01(\mathrm{~s}, 3 \mathrm{H}) ;{ }^{13} \mathrm{C} \mathrm{NMR}\left(101 \mathrm{MHz}, \mathrm{CDCl}_{3}\right) \delta$ $169.9,142.2,141.8,133.2,132.5,128.49,128.46,128.4,128.3,127.6,126.3,126.01,126.00$, 123.6, 123.0, 84.0, 44.1, 30.4, 25.3, 22.4; FTIR (NaCl/thin film) 3059,3 025, 2937, 1734, 1717 , 1652, 1558, 1506, 1244, $747 \mathrm{~cm}^{-1}$; HRMS (LIFDI) [M]+ calculated for $\mathrm{C}_{22} \mathrm{H}_{22} \mathrm{O}_{2}: 318.1620$, found: 318.1648 .

Please note: The absolute configuration of $\mathbf{1 f}$ is tentatively assigned. The absolute configuration resulting from the method used in the preparation of its alcohol precursor S-1f has not been reported in the literature. Please see the experimental for $\mathbf{S - 1 f}$ below.<smiles>CCC(CC)(OC(C)=O)c1ccc2ccccc2c1</smiles>

(S)-1-(3-Methoxyphenyl)-3-(naphthalen-2-yl)pentan-3-yl acetate $((S)-1 h)$. Prepared via General Procedure B using S-1h (89\% ee) as a colorless oil (80\%). The enantiomeric excess was assumed to be $89 \%$ based on the starting material. $[\alpha]_{\mathrm{D}}{ }^{24}=+30.5^{\circ}\left(\mathrm{c} 1.50, \mathrm{CHCl}_{3}\right)$ : ${ }^{1} \mathrm{H}$ NMR $\left(600 \mathrm{MHz}, \mathrm{CDCl}_{3}\right) \delta 7.91-7.84(\mathrm{~m}, 4 \mathrm{H}), 7.55-7.46(\mathrm{~m}, 3 \mathrm{H}), 7.16(\mathrm{t}, J=7.9 \mathrm{~Hz}, 1 \mathrm{H}), 6.70$ $(\mathrm{ddd}, J=13.7,7.8,2.0 \mathrm{~Hz}, 2 \mathrm{H}), 6.65-6.61(\mathrm{~m}, 1 \mathrm{H}), 3.76(\mathrm{~s}, 3 \mathrm{H}), 2.97-2.85(\mathrm{~m}, 1 \mathrm{H}), 2.62(\mathrm{dq}$, $J=14.6,7.3 \mathrm{~Hz}, 1 \mathrm{H}), 2.51-2.41(\mathrm{~m}, 2 \mathrm{H}), 2.39-2.29(\mathrm{~m}, 1 \mathrm{H}), 2.27-2.16(\mathrm{~m}, 4 \mathrm{H}), 0.74(\mathrm{t}, J=$ $7.4 \mathrm{~Hz}, 3 \mathrm{H}) ;{ }^{13} \mathrm{C}$ NMR $\left(151 \mathrm{MHz}, \mathrm{CDCl}_{3}\right) \delta 169.7,159.7,143.6,140.6,133.2,132.5,129.4$, $128.4,128.2$, 127.6, 126.3, 126.0, 124.5, 123.2, 120.9, 114.2, 111.3, 87.8, 55.2, 39.4, 30.9, 30.1, 22.2, 7.8; FTIR (NaCl/thin film) 3056, 2970, 2937, 1733, 1600, 1489, 1455, 1366, 1242, 1046, $1021,819,748 \mathrm{~cm}^{-1}$; HRMS (LIFDI) [M]+ calculated for $\mathrm{C}_{24} \mathrm{H}_{26} \mathrm{O}_{3}: 362.1882$, found: 362.1906 . 
Please note: The absolute configuration of $\mathbf{1 h}$ is tentatively assigned. The absolute configuration resulting from the method used in the preparation of its alcohol precursor $\mathbf{S}-\mathbf{1 h}$ has not been reported in the literature. Please see the experimental for $\mathbf{S}-\mathbf{1 h}$ below.<smiles>CC(=O)OC(C)(c1ccccc1)c1ccc2ccccc2c1</smiles>

(R)-1-(Naphthalen-2-yl)-1-phenylethyl acetate $((\boldsymbol{R})-1 \mathrm{i})$. Prepared via General Procedure B using S-1i (96\% ee) as a colorless sticky oil (54\%). The enantiomeric excess was determined to be $96 \%$ by chiral HPLC analysis (CHIRLPAK IB, $1.0 \mathrm{~mL} / \mathrm{min}, 1.0 \% i$-PrOH/hexanes, $\lambda=254$ $\mathrm{nm}) ; \mathrm{t}_{\mathrm{R}}($ major $)=8.923 \mathrm{~min}, \mathrm{t}_{\mathrm{R}}($ minor $)=7.511 \mathrm{~min} .[\alpha]_{\mathrm{D}}{ }^{24}=+15.3^{\circ}\left(\mathrm{c} 4.1, \mathrm{CHCl}_{3}\right):{ }^{1} \mathrm{H} \mathrm{NMR}$ $\left(600 \mathrm{MHz}, \mathrm{CDCl}_{3}\right) \delta 7.89-7.87(\mathrm{~m}, 1 \mathrm{H}), 7.84(\mathrm{dd}, J=7.4,1.8 \mathrm{~Hz}, 1 \mathrm{H}), 7.79(\mathrm{dd}, J=7.4,1.7$ $\mathrm{Hz}, 1 \mathrm{H}), 7.75(\mathrm{~d}, J=8.7 \mathrm{~Hz}, 1 \mathrm{H}), 7.50-7.44(\mathrm{~m}, 2 \mathrm{H}), 7.38-7.29(\mathrm{~m}, 5 \mathrm{H}), 7.26-7.23(\mathrm{~m}, 1 \mathrm{H})$, $2.30(\mathrm{~s}, 3 \mathrm{H}), 2.16(\mathrm{~s}, 3 \mathrm{H}) ;{ }^{13} \mathrm{C} \mathrm{NMR}\left(151 \mathrm{MHz}, \mathrm{CDCl}_{3}\right) \delta 169.4,145.6,142.9,133.0,132.6$, $128.5,128.3,128.1,127.7,127.3,126.3,126.2,126.1,124.6,124.5,84.8,27.0,22.6$; FTIR ( $\mathrm{NaCl} /$ thin film) 3056, 3024, 2981, 1739, 1368, 1241, 1188, 749, $699 \mathrm{~cm}^{-1}$; HRMS (LIFDI) $[\mathrm{M}]+$ calculated for $\mathrm{C}_{20} \mathrm{H}_{18} \mathrm{O}_{2}: 290.1307$, found: 290.1328 .

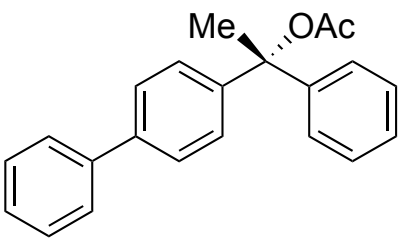

(R)-1-([1,1'-Biphenyl]-4-yl)-1-phenylethyl acetate $((R)-1 j)$. Prepared via General Procedure B using $\mathbf{S - 1 \mathbf { j }}(91 \%$ ee) as a colorless oil $(61 \%)$. The enantiomeric excess was assumed to be $91 \%$ based on the starting material. $[\alpha]_{\mathrm{D}}{ }^{24}=-17.8^{\circ}\left(\mathrm{c} 0.84, \mathrm{CHCl}_{3}\right):{ }^{1} \mathrm{H} \mathrm{NMR}\left(600 \mathrm{MHz}, \mathrm{CDCl}_{3}\right) \delta$ $7.61-7.57(\mathrm{~m}, 2 \mathrm{H}), 7.57-7.53(\mathrm{~m}, 2 \mathrm{H}), 7.44(\mathrm{t}, J=7.7 \mathrm{~Hz}, 2 \mathrm{H}), 7.42-7.32(\mathrm{~m}, 7 \mathrm{H}), 7.29-$ $7.26(\mathrm{~m}, 1 \mathrm{H}), 2.25(\mathrm{~s}, 3 \mathrm{H}), 2.16(\mathrm{~s}, 3 \mathrm{H}) ;{ }^{13} \mathrm{C} \mathrm{NMR}\left(151 \mathrm{MHz}, \mathrm{CDCl}_{3}\right) \delta 169.4,145.7,144.8$, $140.8,140.1,128.9,128.3,127.4,127.3,127.2,127.0,126.5,126.0,84.6,27.0,22.6$; FTIR ( NaCl/thin film) 3057, 3029, 2939, 1739, 1600, 1582, 1487, 1446, 1368, 1238, 1057, 875, 761 $\mathrm{cm}^{-1}$; HRMS (LIFDI) [M]+ calculated for $\mathrm{C}_{22} \mathrm{H}_{20} \mathrm{O}_{2}: 316.1463$, found: 316.1485 . 


\section{Preparation of $(S, E)-2-($ Naphthalen-2-yl)-5-(o-tolyl)pent-4-en-2-yl acetate ((S)-1g).}

$(R)$-BINOL $30 \mathrm{~mol} \%$
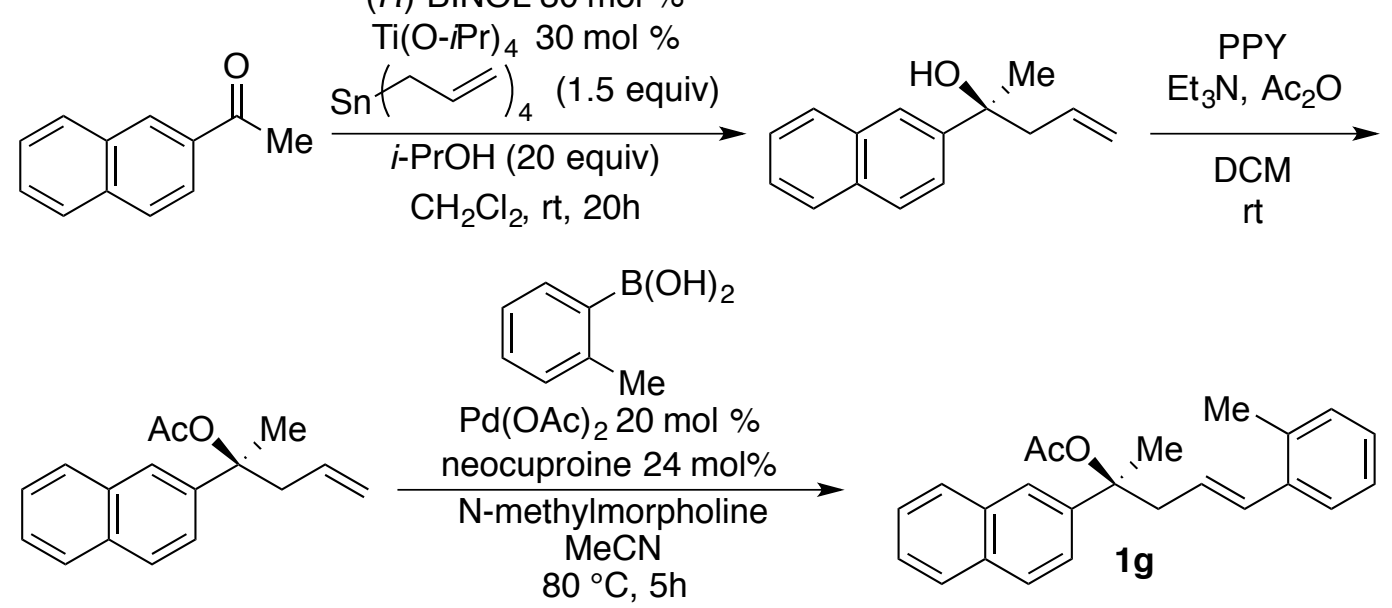

2-(Naphthalen-2-yl)pent-4-en-2-ol. This procedure was adapted from that reported in the literature. ${ }^{4}$ In an oven-dried, $50-\mathrm{mL}$, round-bottomed flask was placed $(R)$-BINOL (312 mg, 1.09 mmol, 0.300 equiv) in $\mathrm{CH}_{2} \mathrm{Cl}_{2}(9.0 \mathrm{~mL})$. $\mathrm{Ti}(\mathrm{O}-i \mathrm{Pr})_{4}(0.33 \mathrm{~mL}, 1.1 \mathrm{mmol}, 0.30$ equiv $)$ was added at room temperature. The mixture was stirred for $10 \mathrm{~min}$. Then $i \mathrm{PrOH}(5.6 \mathrm{~mL}, 73 \mathrm{mmol}$, 20 equiv) was added, followed by a solution of 2-acetonaphthone $(618 \mathrm{mg}, 3.63 \mathrm{mmol}, 1.00$ equiv) and $\mathrm{CH}_{2} \mathrm{Cl}_{2}(3.0 \mathrm{~mL})$, and then tetraallyltin $(0.96 \mathrm{~mL}, 4.0 \mathrm{mmol}, 1.1$ equiv). The orange solution was stirred at room temperature for $22 \mathrm{~h}$ and quenched with sat. $\mathrm{NaHCO}_{3}(40 \mathrm{~mL})$. To remove solids, the mixture was filtered through Celite ${ }^{\circledR}$, which was then washed with $\mathrm{CH}_{2} \mathrm{Cl}_{2}$. The layers were separated, and the organic layer was washed with sat. $\mathrm{NaCl}$, dried $\left(\mathrm{Na}_{2} \mathrm{SO}_{4}\right)$, filtered, and concentrated. The crude mixture was purified on silica gel chromatography $(0-20 \%$ EtOAc/hexanes) to give a 2-(naphthalen-2-yl)pent-4-en-2-ol as a clear oil (634.2 mg, 82\%). The enantiomeric excess was determined to be $96 \%$ by chiral HPLC analysis (CHIRALPAK IC, 1 $\mathrm{mL} / \mathrm{min}, 3 \% \mathrm{i}-\mathrm{PrOH} /$ hexane, $\lambda=254 \mathrm{~nm}) ; \mathrm{t}_{\mathrm{R}}($ major $)=12.164 \mathrm{~min}, \mathrm{t}_{\mathrm{R}}($ minor $)=10.175 \mathrm{~min}$. The spectral data of this compound matches of that reported in the literature. ${ }^{5}$

Please note: The absolute configuration of 2-(naphthalene-2-yl)pent-4-en-2-ol is tentatively assigned. The absolute configuration resulting from this allylation procedure has not been reported in the literature.[REF: Walsh ACIE 2002]

2-(Naphthalen-2-yl)pent-4-en-2-yl acetate. Using General Procedure B, 2-(naphthalen2-yl)pent-4-en-2-yl acetate was obtained as a colorless oil $(632.5 \mathrm{mg}, 86 \%)$ from 2-(naphthalen2-yl)pent-4-en-2-ol (611 mg, $2.88 \mathrm{mmol}, 96 \%$ ee): ${ }^{1} \mathrm{H}$ NMR $\left(600 \mathrm{MHz}, \mathrm{CDCl}_{3}\right) \delta 7.90-7.80$ $(\mathrm{m}, 3 \mathrm{H}), 7.79-7.72(\mathrm{~m}, 1 \mathrm{H}), 7.46(\mathrm{dtd}, J=9.2,6.9,5.4 \mathrm{~Hz}, 3 \mathrm{H}), 5.63(\mathrm{ddt}, J=17.3,10.2,7.2$ $\mathrm{Hz}, 1 \mathrm{H}), 5.11-4.95(\mathrm{~m}, 2 \mathrm{H}), 2.94(\mathrm{dd}, J=14.0,7.1 \mathrm{~Hz}, 1 \mathrm{H}), 2.85$ (dd, $J=14.0,7.3 \mathrm{~Hz}, 1 \mathrm{H})$, $2.09(\mathrm{~s}, 3 \mathrm{H}), 1.91(\mathrm{~s}, 3 \mathrm{H}) ;{ }^{13} \mathrm{C} \mathrm{NMR}\left(151 \mathrm{MHz}, \mathrm{CDCl}_{3}\right) \delta 169.8,142.2,133.2,133.0,132.6$, 128.4, 128.2, 127.6, 126.2, 126.0, 123.6, 123.1, 118.8, 83.4, 46.4, 25.1, 22.4.

$(S, E)-2-(N a p h t h a l e n-2-y l)-5-(o-t o l y l) p e n t-4-e n-2-y l$ acetate $((S)-1 g)$. The procedure was adapted from reported literature. ${ }^{6}$ In $25-\mathrm{mL}$, round-bottomed flask was placed 2(naphthalen-2-yl)pent-4-en-2-yl acetate (632 mg, $2.49 \mathrm{mmol}, 1.00$ equiv), 2-methylphenyl boronic acid (677 mg, $5.00 \mathrm{mmol}, 2.00$ equiv), $N$-methylmorpholine $(0.55 \mathrm{~mL}, 5.0 \mathrm{mmol}, 2.0$ equiv), and $\mathrm{MeCN}$ (10 mL). The flask was exposed to open air. $\mathrm{Pd}(\mathrm{OAc})_{2}$ (335 mg, $0.498 \mathrm{mmol}$, $20 \mathrm{~mol} \mathrm{\%}$ ) and neocuproine (125 $\mathrm{mg}, 0.598 \mathrm{mmol}, 24 \mathrm{~mol} \%$ ) were added. The mixture was heated at $80{ }^{\circ} \mathrm{C}$ for $22 \mathrm{~h}$. The mixture was cooled to room temperature and diluted with $\mathrm{CH}_{2} \mathrm{Cl}_{2}$ $(30 \mathrm{~mL})$. The solid was removed by filtration through a pad of Celite ${ }^{\circledR}$, and the organic layer was 
concentrated. The crude mixture was purified via silica gel chromatography $(0-15 \%$ EtOAc/hexanes) to give $1 \mathrm{~g}$ as a colorless oil (351 $\mathrm{mg}, 41 \%)$. The enantiomeric excess was determined to be $96 \%$ by chiral HPLC analysis (CHIRALPAK IA, $0.8 \mathrm{~mL} / \mathrm{min}, 1 \% i$ $\mathrm{PrOH} /$ hexane, $\lambda=254 \mathrm{~nm}) ; \mathrm{t}_{\mathrm{R}}($ major $)=15.924 \mathrm{~min}, \mathrm{t}_{\mathrm{R}}($ minor $)=21.866 \mathrm{~min} .[\alpha]_{\mathrm{D}}{ }^{24}=+10.7^{\circ}(\mathrm{c}$ 1.12, $\left.\mathrm{CHCl}_{3}\right):{ }^{1} \mathrm{H}$ NMR $\left(600 \mathrm{MHz}, \mathrm{CDCl}_{3}\right) \delta 7.85-7.78(\mathrm{~m}, 4 \mathrm{H}), 7.52-7.44(\mathrm{~m}, 3 \mathrm{H}), 7.28(\mathrm{dd}$, $J=7.4,2.2 \mathrm{~Hz}, 1 \mathrm{H}), 7.14-7.07(\mathrm{~m}, 3 \mathrm{H}), 6.62-6.56(\mathrm{~m}, 1 \mathrm{H}), 5.87(\mathrm{dt}, J=15.3,7.4 \mathrm{~Hz}, 1 \mathrm{H})$, $3.12-2.99(\mathrm{~m}, 2 \mathrm{H}), 2.22(\mathrm{~s}, 3 \mathrm{H}), 2.10(\mathrm{~s}, 3 \mathrm{H}), 1.97(\mathrm{~s}, 3 \mathrm{H}) ;{ }^{13} \mathrm{C} \mathrm{NMR}\left(151 \mathrm{MHz}, \mathrm{CDCl}_{3}\right) \delta$ $169.8,142.2$, 136.8, 135.3, 133.2, 132.6, 132.1, 130.2, 128.4, 128.2, 127.6, 127.3, 126.3, 126.1, 126.05, 126.03, 125.9, 123.6, 123.2, 83.8, 45.9, 25.3, 22.4, 19.8; FTIR (NaCl/thin film) 2955, 2921, 2850, 1713, 1464, 1364, 1232, 1076, $748 \mathrm{~cm}^{-1}$; HRMS (LIFDI) [M]+ calculated for $\mathrm{C}_{24} \mathrm{H}_{24} \mathrm{O}_{2}: 344.1776$, found: 344.1769 .

Please note: The absolute configuration of $\mathbf{1 g}$ is tentatively assigned. The absolute configuration resulting from the method used in the preparation of its alcohol precursor has not been reported in the literature, as discussed above.

\section{Preparation of Tertiary Benzyl Alcohols (S-1a - S-1j).}

In addition to the reactions described below, a number of other methods are available for the preparation of highly enantioenriched tertiary benzylic alcohols. Notable examples are described in the following references:

1) Jeon, S.-J., Li, H. \& Walsh, P. J. A Green Chemistry Approach to a More Efficient Asymmetric Catalyst: Solvent-Free and Highly Concentrated Alkyl Additions to Ketones. J. Am. Chem. Soc. 127, 16416-16425 (2005).

2) García, C., LaRochelle, L. K. \& Walsh, P. J. A Practical Catalytic Asymmetric Addition of Alkyl Groups to Ketones. J. Am. Chem. Soc. 124, 10970-10971 (2002).

3) Li, H. \& Walsh, P. J. Catalytic Asymmetric Vinylation of Ketones. J. Am. Chem. Soc. 126, 6538-6539 (2004).

4) García, C. \& Walsh, P. J. Highly Enantioselective Catalytic Phenylation of Ketones with a Constrained Geometry Titanium Catalyst. Org. Lett. 5, 3641-3644 (2003).

5) Waltz, K. M., Gavenonis, J. \& Walsh, P. J. A Simple, Reliable, Catalytic Asymmetric Allylation of Ketones. Angew. Chem., Int. Ed. 41, 3697-3699 (2002).

6) Watson, C. G. \& Aggarwal, V. K. Asymmetric Synthesis of 1-Heteroaryl-1-arylalkyl Tertiary Alcohols and 1-Pyridyl-1-arylethanes by Lithiation-Borylation Methodology. Org. Lett. 15, 1346-1349 (2013).

7) Cozzi, P. G. Enantioselective Alkynylation of Ketones Catalyzed by Zn(Salen) Complexes. Angew. Chem., Int. Ed. 42, 2895-2898 (2003).

8) Zhou, Y., Wang, R., Xu, Z., Yan, W., Liu, L., Kang, Y. \& Han, Z. Highly Enantioselective Phenylacetylene Additions to Ketones Catalyzed by (S)-BINOL-Ti Complex. Org. Lett. 6, 4147-4149 (2004). 
Preparation of S-1a - S-1d.
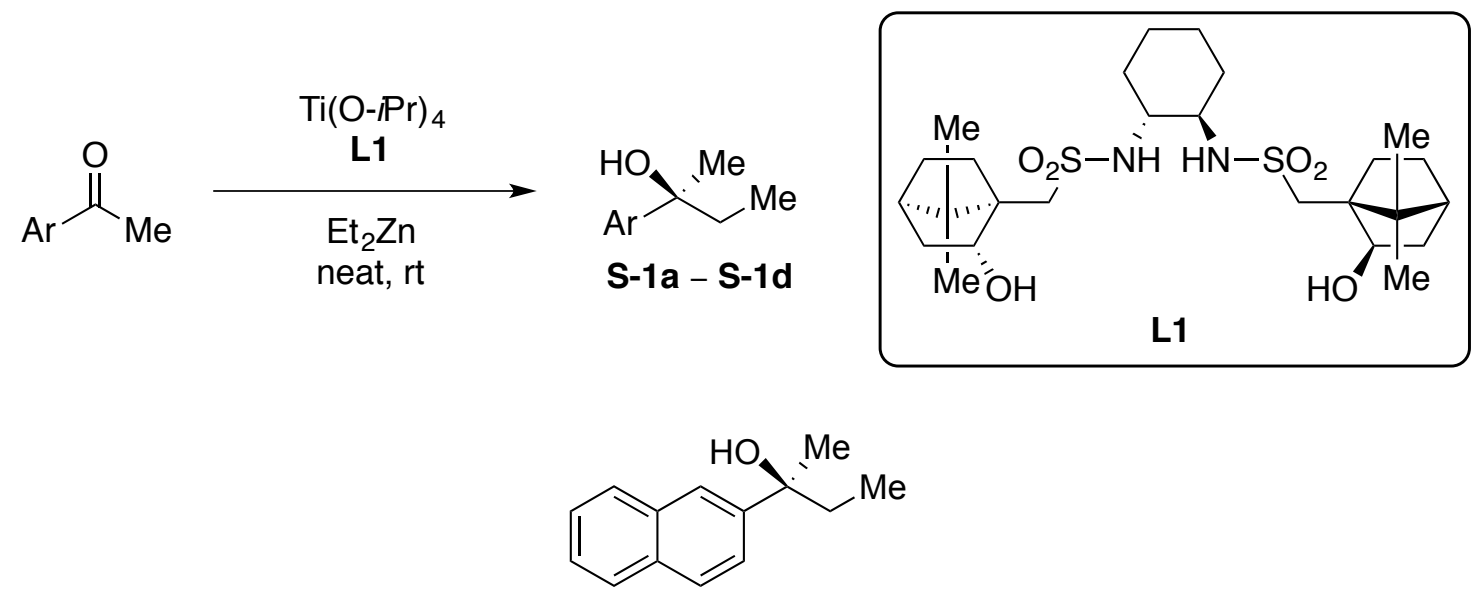

(S)-2-(Naphthalen-2-yl)butan-2-ol ((S)-S-1a). This procedure was adapted from that reported in the literature. ${ }^{7 \mathrm{a}}$ In an oven-dried, $100-\mathrm{mL}$, round-bottomed flask was placed $\mathbf{L 1}$ (33 mg, 0.060 mmol, 0.010 equiv) and $\mathrm{Et}_{2} \mathrm{Zn}(0.73 \mathrm{~mL}, 7.2 \mathrm{mmol}, 1.2 \text { equiv). Ti(O-iPr })_{4}(2.1 \mathrm{~mL}, 7.2 \mathrm{mmol}$, 1.2 equiv) was added. The resulting greenish solution was stirred at room temperature for $5 \mathrm{~min}$. 2-Acetonaphthalone ( $1.02 \mathrm{~g}, 6.00 \mathrm{mmol}, 1.00$ equiv) was added into the flask in one portion. The mixture was stirred at room temperature for $17 \mathrm{~h}$. The resulting brown sticky oil was diluted with EtOAc $(50 \mathrm{~mL})$ and quenched with $\mathrm{HCl}(1 \mathrm{~N})$. The product was extracted from the aqueous layer with EtOAc $(25 \mathrm{~mL} \times 2)$. The combined organic layers were washed with sat. $\mathrm{NaCl}$, dried $\left(\mathrm{MgSO}_{4}\right)$, filtered, and concentrated. The residue was purified via silica gel chromatography $\left(5-10 \% \mathrm{Et}_{2} \mathrm{O} /\right.$ hexanes$)$ to give $(\boldsymbol{S})-\mathrm{S}-1 \mathbf{a}(470 \mathrm{mg}, 39 \%)$ as a colorless oil. The enantiomeric excess was determined to be $97 \%$ by chiral HPLC analysis (CHIRLPAK IB, $1.0 \mathrm{~mL} / \mathrm{min}, 3.0 \%$ $i$-PrOH$/$ hexanes, $\lambda=254 \mathrm{~nm}$ ); $\mathrm{t}_{\mathrm{R}}$ (major) $=10.269 \mathrm{~min}, \mathrm{t}_{\mathrm{R}}$ (minor) $=11.370 \mathrm{~min}$. Based on the optical rotation, $[\alpha]_{\mathrm{D}}{ }^{24}=-9.5^{\circ}(\mathrm{c} 1.0, \mathrm{MeOH})\left(\right.$ Literature data: $[\alpha]_{\mathrm{D}}{ }^{24}=+16.3^{\circ}(\mathrm{c} 1.0, \mathrm{MeOH})$ for $R$ configuration), ${ }^{7 \mathrm{~b}}$ the absolute configuration of $\mathbf{S - 1 a}$ was assigned as $S$. The spectral data of this compound matched that reported in the literature. ${ }^{7}$<smiles>CCC(C)(O)c1ccc2cc(OC)ccc2c1</smiles>

(S)-2-(6-Methoxynaphthalen-2-yl)butan-2-ol ((S)-S-1b). Prepared via the procedure described above for preparation of $(\boldsymbol{S})-\mathbf{S}-\mathbf{1 a}$ as a colorless oil (32\%). The enantiomeric excess was determined to be $92 \%$ by chiral HPLC analysis (CHIRLPAK IC, $1.0 \mathrm{~mL} / \mathrm{min}, 3.0 \% i$ $\mathrm{PrOH} / \mathrm{h}$ exanes, $\lambda=254 \mathrm{~nm}) ; \mathrm{t}_{\mathrm{R}}$ (major) $=21.219 \mathrm{~min}, \mathrm{t}_{\mathrm{R}}($ minor $)=16.516 \mathrm{~min} .[\alpha]_{\mathrm{D}}{ }^{24}=+5.1^{\circ}(\mathrm{c}$ 3.02, $\mathrm{CHCl}_{3}$ ): ${ }^{1} \mathrm{H}$ NMR $\left(600 \mathrm{MHz}, \mathrm{CDCl}_{3}\right) \delta 7.83(\mathrm{~d}, J=1.9 \mathrm{~Hz}, 1 \mathrm{H}), 7.73(\mathrm{dd}, J=12.2,8.7 \mathrm{~Hz}$, 2H), $7.50(\mathrm{dd}, J=8.6,1.9 \mathrm{~Hz}, 1 \mathrm{H}), 7.16-7.11(\mathrm{~m}, 2 \mathrm{H}), 3.92(\mathrm{~s}, 3 \mathrm{H}), 1.99-1.85(\mathrm{~m}, 2 \mathrm{H}), 1.79$ $(\mathrm{d}, J=3.6 \mathrm{~Hz}, 1 \mathrm{H}), 1.63(\mathrm{~s}, 3 \mathrm{H}), 0.82(\mathrm{td}, J=7.4,1.0 \mathrm{~Hz}, 3 \mathrm{H}) ;{ }^{13} \mathrm{C} \mathrm{NMR}\left(151 \mathrm{MHz}, \mathrm{CDCl}_{3}\right) \delta$ 157.7, 143.0, 133.4, 129.7, 128.8, 126.8, 124.4, 123.3, 118.9, 105.7, 75.2, 55.5, 36.7, 29.9, 8.5; FTIR (NaCl/thin film) 3447 (br s), 3059, 2969, 2935, 1634, 4606, 1504, 1485, 1462, 1388, 1265 , $1199,1033,852,810 \mathrm{~cm}^{-1}$; HRMS (CI+) [M]+H calculated for $\mathrm{C}_{15} \mathrm{H}_{19} \mathrm{O}_{2}: 231.1385$, found: 231.1400 . 


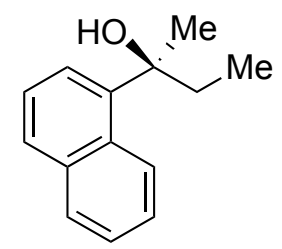

(S)-2-(Naphthalen-1-yl)butan-2-ol ((S)-S-1c). Prepared via the procedure described above for preparation of $(\boldsymbol{S})$-S-1a as a colorless oil (9\%). The enantiomeric excess was determined as $90 \%$ by chiral HPLC analysis (CHIRLPAK IB, $0.7 \mathrm{~mL} / \mathrm{min}, 2.0 \% i$-PrOH/hexanes, $\lambda=254 \mathrm{~nm}$ ); $\mathrm{t}_{\mathrm{R}}($ major $)=15.085 \mathrm{~min}, \mathrm{t}_{\mathrm{R}}($ minor $)=17.287 \mathrm{~min} .[\alpha]_{\mathrm{D}}{ }^{24}=+33.7^{\circ}\left(\mathrm{c} 1.91, \mathrm{CHCl}_{3}\right):{ }^{1} \mathrm{H} \mathrm{NMR}(600$ $\left.\mathrm{MHz}, \mathrm{CDCl}_{3}\right) \delta 8.79-8.75(\mathrm{~m}, 1 \mathrm{H}), 7.87(\mathrm{dd}, J=7.8,1.9 \mathrm{~Hz}, 1 \mathrm{H}), 7.77(\mathrm{~d}, J=8.2 \mathrm{~Hz}, 1 \mathrm{H})$, $7.58(\mathrm{dd}, J=7.3,1.3 \mathrm{~Hz}, 1 \mathrm{H}), 7.48(\mathrm{pd}, J=6.8,1.6 \mathrm{~Hz}, 2 \mathrm{H}), 7.42(\mathrm{t}, J=7.7 \mathrm{~Hz}, 1 \mathrm{H}), 2.30-2.17$ $(\mathrm{m}, 2 \mathrm{H}), 2.02(\mathrm{~s}, 1 \mathrm{H}), 1.82(\mathrm{~s}, 3 \mathrm{H}), 0.83(\mathrm{t}, J=7.5 \mathrm{~Hz}, 3 \mathrm{H}) ;{ }^{13} \mathrm{C}$ NMR $\left(151 \mathrm{MHz}, \mathrm{CDCl}_{3}\right) \delta$ 142.4, 135.0, 131.1, 129.3, 128.6, 127.1, 125.3, 125.2, 124.9, 124.0, 76.9, 35.4, 29.5, 9.0; FTIR ( NaCl/thin film) 3420 (brs), 3048, 2971, 2936, 2877, 1653, 1508, 1456, 1374, 1117, 804, 777 $\mathrm{cm}^{-1}$; HRMS (EI+) [M]+H calculated for $\mathrm{C}_{14} \mathrm{H}_{16} \mathrm{O}: 200.1201$, found: 200.1205 .<smiles>CCC(C)(O)c1ccc2nc(C)ccc2c1</smiles>

(S)-2-(2-Methylquinolin-6-yl)butan-2-ol ( $(S)-S-1 d)$. Prepared via the procedure described above for preparation of $(\boldsymbol{S})-\mathbf{S}-\mathbf{1 a}$ as a pale yellow solid (mp $\left.86-89^{\circ}, 41 \%\right)$. The enantiomeric excess was determined as $99 \%$ by chiral HPLC analysis (CHIRLPAK IC, $1 \mathrm{~mL} / \mathrm{min}, 8.0 \% i$ $\mathrm{PrOH} /$ hexanes, $\lambda=230 \mathrm{~nm}) ; \mathrm{t}_{\mathrm{R}}$ (major) $=23.228 \mathrm{~min}, \mathrm{t}_{\mathrm{R}}($ minor $)=19.313 \mathrm{~min}$. $[\alpha]_{\mathrm{D}}{ }^{24}=+33^{\circ}(\mathrm{c}$ 1.03, $\left.\mathrm{CHCl}_{3}\right):{ }^{1} \mathrm{H} \mathrm{NMR}\left(600 \mathrm{MHz}, \mathrm{CDCl}_{3}\right) \delta 8.04(\mathrm{~d}, J=8.4 \mathrm{~Hz}, 1 \mathrm{H}), 7.98(\mathrm{~d}, J=8.8 \mathrm{~Hz}, 1 \mathrm{H})$, $7.85(\mathrm{~d}, J=2.1 \mathrm{~Hz}, 1 \mathrm{H}), 7.71(\mathrm{dd}, J=8.9,2.1 \mathrm{~Hz}, 1 \mathrm{H}), 7.28(\mathrm{~d}, J=8.4 \mathrm{~Hz}, 1 \mathrm{H}), 2.74(\mathrm{~s}, 3 \mathrm{H})$, $1.93(\mathrm{ddt}, J=27.2,14.1,7.1 \mathrm{~Hz}, 2 \mathrm{H}), 1.64(\mathrm{~s}, 3 \mathrm{H}), 0.81(\mathrm{t}, J=7.5 \mathrm{~Hz}, 3 \mathrm{H}) ;{ }^{13} \mathrm{C} \mathrm{NMR}(151 \mathrm{MHz}$, $\left.\mathrm{CDCl}_{3}\right) \delta 158.9,147.0,145.1,136.5,128.6,127.4,126.2,123.1,122.3,75.1,36.7,30.0,25.5$, 8.4; FTIR (NaCl/thin film) 3355 (brs), 2969, 2933, 2878, 1601, 1497, 1457, 1374, 1165, 1126, 837, $755 \mathrm{~cm}^{-1}$; HRMS (CI+) [M]+H calculated for $\mathrm{C}_{14} \mathrm{H}_{18} \mathrm{NO}: 216.1388$, found: 216.1398 .

A crystal suitable for X-ray diffraction analysis was obtained via diffusion of hexanes into a solution of $\mathbf{S - 1 d}$ in EtOAc at $-18{ }^{\circ} \mathrm{C}$. The crystal structure demonstrates that the absolute configuration is $S$ (Figure S3).

Figure S3. Molecular diagram of $(S)$-S-1d with ellipsoids at 50\% probability, all non-oxygen bound H-atoms omitted for clarity. (CCDC 1424634)

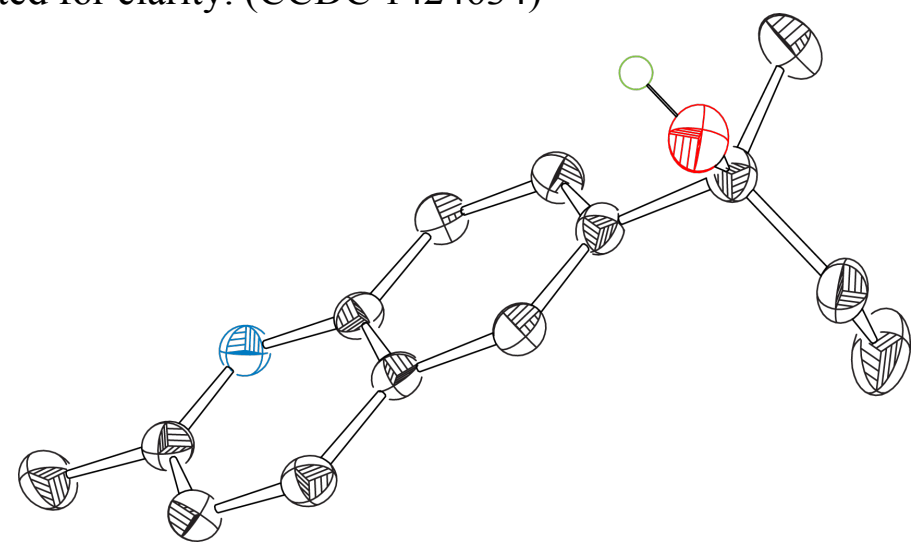


Preparation of S-1e.

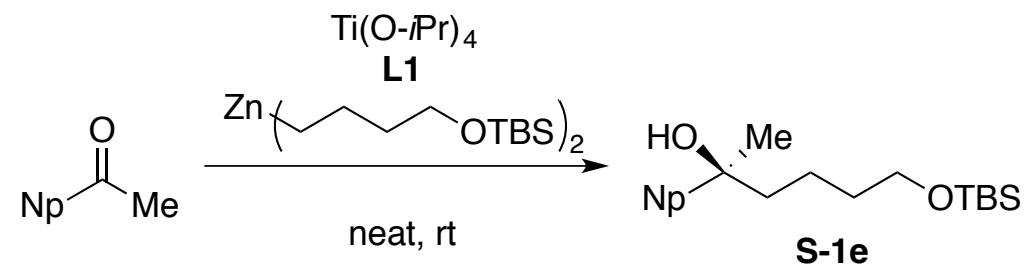

(S)-6-((tert-Butyldimethylsilyl)oxy)-2-(4-nitrophenyl)hexan-2-ol ((S)-S-1e). Prepared via the procedure described above for preparation of $(\boldsymbol{S})$-S-1a, except that bis(4-(tertbutyldimethylsilyl)oxy)butyl)zinc was used instead of $\mathrm{Et}_{2} \mathrm{Zn}$, as a colorless oil (30\%). The enantiomeric excess was determined to be $99 \%$ by chiral HPLC analysis (CHIRLPAK IA, 0.6 $\mathrm{mL} / \mathrm{min}, 3.0 \% i-\mathrm{PrOH} /$ hexanes, $\lambda=254 \mathrm{~nm}) ; \mathrm{t}_{\mathrm{R}}($ major $)=14.468 \mathrm{~min}, \mathrm{t}_{\mathrm{R}}($ minor $)=13.695 \mathrm{~min}$. $[\alpha]_{\mathrm{D}}{ }^{24}=+10.2^{\circ}\left(\mathrm{c} 2.63, \mathrm{CHCl}_{3}\right):{ }^{1} \mathrm{H}$ NMR $\left(600 \mathrm{MHz}, \mathrm{CDCl}_{3}\right) \delta 7.93-7.89(\mathrm{~m}, 1 \mathrm{H}), 7.86-7.79$ $(\mathrm{m}, 3 \mathrm{H}), 7.52(\mathrm{dd}, J=8.6,1.9 \mathrm{~Hz}, 1 \mathrm{H}), 7.50-7.42(\mathrm{~m}, 2 \mathrm{H}), 3.54(\mathrm{t}, J=6.5 \mathrm{~Hz}, 2 \mathrm{H}), 2.01-1.84$ $(\mathrm{m}, 3 \mathrm{H}), 1.65(\mathrm{~s}, 3 \mathrm{H}), 1.53-1.45(\mathrm{~m}, 2 \mathrm{H}), 1.40-1.28(\mathrm{~m}, 1 \mathrm{H}), 1.27-1.17(\mathrm{~m}, 1 \mathrm{H}), 0.83(\mathrm{~s}$, 9H), $-0.01(\mathrm{~d}, J=2.3 \mathrm{~Hz}, 6 \mathrm{H}) ;{ }^{13} \mathrm{C}$ NMR $\left(151 \mathrm{MHz}, \mathrm{CDCl}_{3}\right) \delta 145.5,133.3,132.4,128.3,128.0$, 127.6, 126.1, 125.8, 123.8, 123.3, 75.1, 63.1, 43.8, 33.1, 30.4, 26.1, 20.6, 18.4, -5.16, -5.17; FTIR (NaCl/thin film) 3432 (brs), 3056, 2952, 2929, 2857, 1471, 1254, 1101, 836, 775, $476 \mathrm{~cm}^{-}$ ${ }^{1}$; HRMS (LIFDI) [M]+ calculated for $\mathrm{C}_{24} \mathrm{H}_{26} \mathrm{O}_{3}: 358.2328$, found: 358.2343 .

Preparation of S-1f and S-1h.

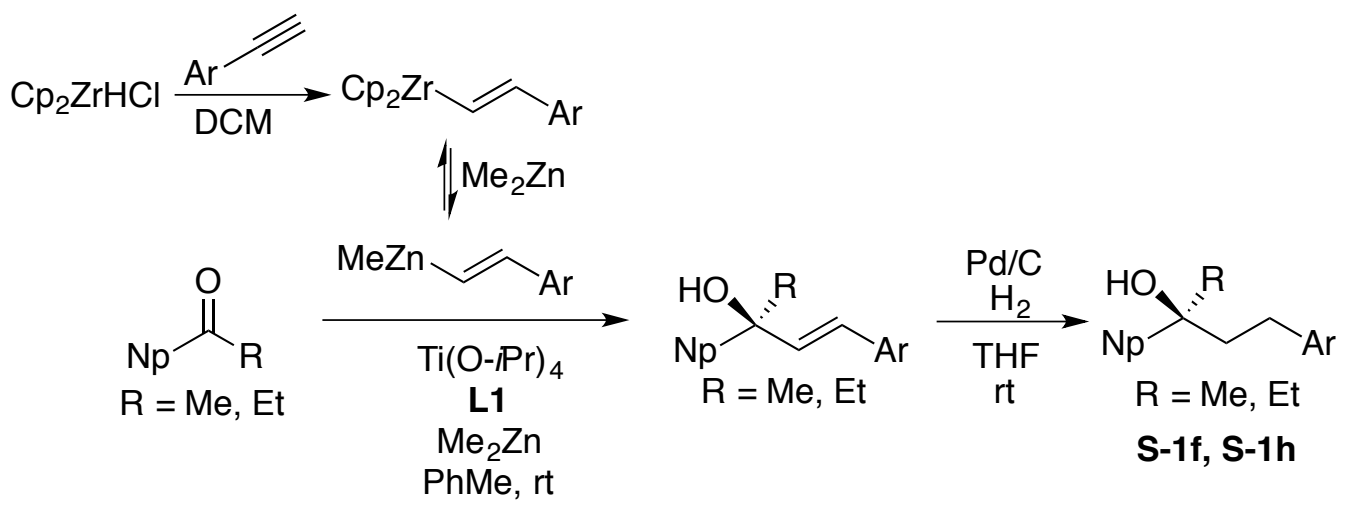<smiles>CC(O)(CCc1ccccc1)c1ccc2ccccc2c1</smiles>

$(S)$-2-(naphthalen-2-yl)-4-phenylbutan-2-ol $((S)-S-1 f)$. The procedure for formation of the allylic alcohol was adapted from a reported procedure. ${ }^{8}$ For preparation the vinylzinc reagent, in an oven-dried, round-bottomed flask was placed $\mathrm{Cp}_{2} \mathrm{ZrHCl}$ (346 $\mathrm{mg}, 1.20 \mathrm{mmol}, 1.20$ equiv) and $\mathrm{CH}_{2} \mathrm{Cl}_{2}(4.0 \mathrm{~mL})$. At room temperature, phenylacetylene $(0.13 \mathrm{~mL}, 1.2 \mathrm{mmol}, 1.2$ equiv) was added into the flask and stirred for $10 \mathrm{~min}$. The solvent was removed, and the orange solid was dissolved in $\mathrm{PhMe}(4.0 \mathrm{~mL})$. The solution was cooled to $-78{ }^{\circ} \mathrm{C}$, before $\mathrm{Me}_{2} \mathrm{Zn}(1.0 \mathrm{~mL}, 1.2$ mmol, 1.2 equiv, $1.2 \mathrm{M}$ in PhMe) was added. The mixture was stirred at $-78^{\circ} \mathrm{C}$ for $10 \mathrm{~min}$. The resulting solution was assumed to be the vinylzinc in PhMe solution. In a separate flask was placed $\mathbf{L 1}\left(54.5 \mathrm{mg}, 0.100 \mathrm{mmol}, 0.100\right.$ equiv), $\mathrm{Me}_{2} \mathrm{Zn}(0.33 \mathrm{~mL}, 0.40 \mathrm{mmol}, 0.40$ equiv, $1.2 \mathrm{M}$ in $\mathrm{PhMe})$, and $\mathrm{PhMe}(2.0 \mathrm{~mL})$. To this mixture was added $\mathrm{Ti}(\mathrm{O}-i \mathrm{Pr})_{4}(0.36 \mathrm{~mL}, 1.2 \mathrm{mmol}, 1.2$ 
equiv) at room temperature. The mixture was stirred at room temperature for $15 \mathrm{~min}$, then the solution was added into the pre-formed vinylzinc solution at $-78{ }^{\circ} \mathrm{C}$ via cannula. The combined solution was warmed to $0^{\circ} \mathrm{C}$, and treated with a solution of 2-acetylnaphthalone $(170 \mathrm{mg}, 1.0$ mmol, 1.0 equiv) and PhMe $(1.0 \mathrm{~mL})$. The resulting reddish solution was stirred at room temperature for $16 \mathrm{~h}$ and then quenched with sat. $\mathrm{NaHCO}_{3}$ aq. $(20 \mathrm{~mL})$. The solid was removed via filtration through a pad of Celite ${ }^{\circledR}$. The product was extracted with EtOAc. The combined organic layers were washed with sat. $\mathrm{NaCl}$, dried $\left(\mathrm{Na}_{2} \mathrm{SO}_{4}\right)$, filtered, and concentrated. The residue was purified via silica gel chromatography (0-10\% EtOAc/hexanes) to give $(E)-2$ (naphthalen-2-yl)-4-phenylbut-3-en-2-ol as a colorless oil (202 mg, 77\%), which was used directly in next step.

Please note: We assume the same absolute configuration using these homemade vinylzinc reagents as when $\mathrm{Et}_{2} \mathrm{Zn}$ is used. However, the absolute configuration obtained for this procedure has not been reported in the literature. ${ }^{8}$

(E)-2-(Naphthalen-2-yl)-4-phenylbut-3-en-2-ol (202 $\mathrm{mg}, 0.736 \mathrm{mmol})$ was dissolved in THF ( $7 \mathrm{~mL})$ at room temperature. $\mathrm{Pd} / \mathrm{C}(39 \mathrm{mg}, 0.037 \mathrm{mmol}, 10 \% \mathrm{w})$ was added. The headspace of the flask was evacuated and refilled with $\mathrm{H}_{2}$ three times. The mixture was then stirred at room temperature for $12 \mathrm{~h}$ under $\mathrm{H}_{2}(1 \mathrm{~atm})$. The solid was removed via filtration through a tightpacked pad of Celite ${ }^{\circledR}$. The filtrate was concentrated and purified via silica gel chromatography $\left(0-10 \%\right.$ EtOAc/hexanes) to give $(\boldsymbol{S})-\mathbf{S}-1 \mathbf{f}(189 \mathrm{mg}, 93 \%)$ as a white solid $\left(\mathrm{mp} 76-79^{\circ}\right) .[\alpha]_{\mathrm{D}}{ }^{24}=$ $+43.6^{\circ}\left(\mathrm{c} 2.2, \mathrm{CHCl}_{3}\right):{ }^{1} \mathrm{H} \mathrm{NMR}\left(600 \mathrm{MHz}, \mathrm{CDCl}_{3}\right) \delta 8.02-7.98(\mathrm{~m}, 1 \mathrm{H}), 7.92-7.86(\mathrm{~m}, 3 \mathrm{H})$, $7.60(\mathrm{dd}, J=8.6,1.9 \mathrm{~Hz}, 1 \mathrm{H}), 7.52(\mathrm{pd}, J=6.8,1.5 \mathrm{~Hz}, 2 \mathrm{H}), 7.29-7.21(\mathrm{~m}, 2 \mathrm{H}), 7.21-7.16$ $(\mathrm{m}, 1 \mathrm{H}), 7.16-7.13(\mathrm{~m}, 2 \mathrm{H}), 2.69(\mathrm{ddd}, J=13.7,11.8,5.6 \mathrm{~Hz}, 1 \mathrm{H}), 2.48(\mathrm{ddd}, J=13.6,11.8$, $4.9 \mathrm{~Hz}, 1 \mathrm{H}), 2.33-2.20(\mathrm{~m}, 2 \mathrm{H}), 1.93(\mathrm{~s}, 1 \mathrm{H}), 1.73(\mathrm{~s}, 3 \mathrm{H}) ;{ }^{13} \mathrm{C}$ NMR $\left(151 \mathrm{MHz}, \mathrm{CDCl}_{3}\right) \delta$ $145.0,142.3,133.4,132.4,128.51,128.46,128.3,128.2,127.6,126.3,125.90,125.89,123.7$, 123.4, 75.1, 45.9, 30.8, 30.7; FTIR (NaCl/thin film) 3446 (brs), 3057, 3024, 2972, 2932, 1601, $1496,1455,819,747,700,487 \mathrm{~cm}^{-1}$; HRMS (EI+) [M]+ calculated for $\mathrm{C}_{20} \mathrm{H}_{20} \mathrm{O}: 276.1514$, found: 276.1514 .

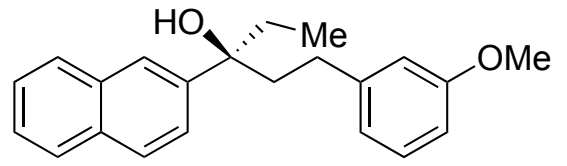

(S)-1-(3-methoxyphenyl)-3-(naphthalen-2-yl)pentan-3-ol ((S)-S-1h). Following a similar procedure as for the preparation of $(\boldsymbol{S})-\mathbf{S}-1 \mathbf{f}$ above, $(\boldsymbol{S})-\mathbf{S}-\mathbf{1 h}$ was prepared as a colorless oil $(40 \%$ overall yield from 1-(naphthalene-2-yl)propan-1-one). The enantiomeric excess was determined to be $89 \%$ ee by chiral HPLC analysis (CHIRLPAK IC, $1.0 \mathrm{~mL} / \mathrm{min}, 5.0 \% i$-PrOH/hexanes, $\lambda=254 \mathrm{~nm}) ; \mathrm{t}_{\mathrm{R}}($ major $)=9.989 \mathrm{~min}, \mathrm{t}_{\mathrm{R}}($ minor $)=10.700 \mathrm{~min} .[\alpha]_{\mathrm{D}}{ }^{24}=+41.3^{\circ}\left(\mathrm{c} 0.92, \mathrm{CHCl}_{3}\right):{ }^{1} \mathrm{H}$ NMR $\left(600 \mathrm{MHz}, \mathrm{CDCl}_{3}\right) \delta 7.98-7.93(\mathrm{~m}, 1 \mathrm{H}), 7.90-7.84(\mathrm{~m}, 3 \mathrm{H}), 7.50(\mathrm{dtd}, J=9.4,7.0,5.3$ $\mathrm{Hz}, 3 \mathrm{H}), 7.17(\mathrm{t}, J=7.9 \mathrm{~Hz}, 1 \mathrm{H}), 6.71(\mathrm{dd}, J=8.0,2.1 \mathrm{~Hz}, 2 \mathrm{H}), 6.66(\mathrm{t}, J=2.0 \mathrm{~Hz}, 1 \mathrm{H}), 3.76(\mathrm{~s}$, $3 \mathrm{H}), 2.66(\mathrm{ddd}, J=13.6,11.7,5.5 \mathrm{~Hz}, 1 \mathrm{H}), 2.35(\mathrm{ddd}, J=13.6,11.8,4.7 \mathrm{~Hz}, 1 \mathrm{H}), 2.32-2.17$ $(\mathrm{m}, 2 \mathrm{H}), 2.03(\mathrm{dq}, J=14.9,7.4 \mathrm{~Hz}, 1 \mathrm{H}), 1.95(\mathrm{dq}, J=14.5,7.4 \mathrm{~Hz}, 1 \mathrm{H}), 1.88(\mathrm{~s}, 1 \mathrm{H}), 0.81(\mathrm{t}, J=$ $7.4 \mathrm{~Hz}, 3 \mathrm{H}) ;{ }^{13} \mathrm{C} \mathrm{NMR}\left(151 \mathrm{MHz}, \mathrm{CDCl}_{3}\right) \delta 159.8,144.2,143.0,133.3,132.3,129.5,128.3$, 128.1, 127.6, 126.2, 125.8, 124.4, 123.9, 120.8, 114.2, 111.2, 77.6, 55.2, 44.5, 35.9, 30.3, 7.9; FTIR (NaCl/thin film) 3486 (brs), 3054, 2964, 2936, 1680, 1489, 1455, 1258, 1152, 1048, 819, $748,698,477 \mathrm{~cm}^{-1}$; HRMS (EI+) [M]+ calculated for $\mathrm{C}_{22} \mathrm{H}_{24} \mathrm{O}_{2}: 320.1776$, found: 320.1753 .

Please note: We assume the same absolute configuration using these homemade vinylzinc reagents as when $\mathrm{Et}_{2} \mathrm{Zn}$ is used. However, the absolute configuration obtained for this procedure has not been reported in the literature. ${ }^{8}$ 
Preparation of S-1i, S-1j.

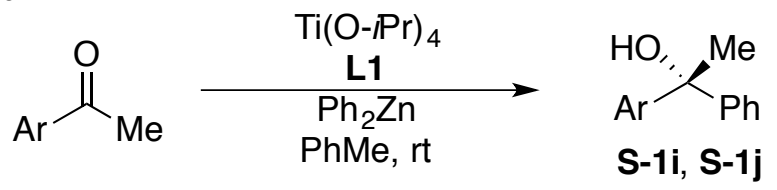<smiles>C[C@](O)(c1ccccc1)c1ccc2ccccc2c1</smiles>

$(R)$-1-(naphthalen-2-yl)-1-phenylethan-1-ol $((\boldsymbol{R})$-S-1i). The procedure was adapted from that reported in the literature. ${ }^{9}$ In an oven-dried, $50-\mathrm{mL}$, round-bottomed flask was placed $\mathbf{L 1}$ (54.5 $\mathrm{mg}, 0.100 \mathrm{mmol}, 0.100$ equiv), $\mathrm{Ph}_{2} \mathrm{Zn}(351 \mathrm{~mL}, 1.60 \mathrm{mmol}, 1.60$ equiv), and $\mathrm{PhMe}(10 \mathrm{~mL})$ at room temperature. $\mathrm{Ti}(\mathrm{O}-i \mathrm{Pr})_{4}(0.18 \mathrm{~mL}, 0.60 \mathrm{mmol}, 0.60$ equiv) was added. The mixture was stirred at room temperature for $15 \mathrm{~min}$. A solution of 2-acetonaphthalone $(170 \mathrm{mg}, 1.0 \mathrm{mmol}, 1.0$ equiv) and $\mathrm{PhMe}(5 \mathrm{~mL})$ was added into the flask. The mixture was stirred at room temperature for $17 \mathrm{~h}$. The reaction was then quenched with sat. $\mathrm{NH}_{4} \mathrm{Cl}$ aq. $(20 \mathrm{~mL})$. The solids were removed via filtration through a pad of Celite ${ }^{\mathbb{R}}$. The mixture was extracted with EtOAc $(25 \mathrm{~mL} \times 2)$. The combined organic layers were washed with sat. $\mathrm{NaCl}$, dried $\left(\mathrm{NaSO}_{4}\right)$, filtered, and concentrated. The residue was purified via silica gel chromatography $\left(5-15 \% \mathrm{Et}_{2} \mathrm{O} /\right.$ hexanes $)$ to give $(\boldsymbol{R})-\mathbf{S}-\mathbf{1 i}$ (203.7 $\mathrm{mg}, 82 \%$ ) as a colorless oil. The enantiomeric excess was determined to be $95 \%$ by chiral HPLC analysis (CHIRLPAK IB, $1.0 \mathrm{~mL} / \mathrm{min}, 4.0 \% i$-PrOH/hexanes, $\lambda=254 \mathrm{~nm}) ; \mathrm{t}_{\mathrm{R}}$ (major) $=$ 13.183 min, $\mathrm{t}_{\mathrm{R}}($ minor $)=14.119$ min. $[\alpha]_{\mathrm{D}}{ }^{24}=+10.7^{\circ}\left(\mathrm{c} 0.82, \mathrm{CHCl}_{3}\right):{ }^{1} \mathrm{H}$ NMR $(600 \mathrm{MHz}$, $\left.\mathrm{CDCl}_{3}\right) \delta 8.00-7.95(\mathrm{~m}, 1 \mathrm{H}), 7.87-7.83(\mathrm{~m}, 1 \mathrm{H}), 7.81(\mathrm{dd}, J=7.5,1.8 \mathrm{~Hz}, 1 \mathrm{H}), 7.77(\mathrm{~d}, J=$ $8.6 \mathrm{~Hz}, 1 \mathrm{H}), 7.52-7.44(\mathrm{~m}, 4 \mathrm{H}), 7.42(\mathrm{dd}, J=8.6,1.9 \mathrm{~Hz}, 1 \mathrm{H}), 7.33(\mathrm{dd}, J=8.5,7.0 \mathrm{~Hz}, 2 \mathrm{H})$, $7.27(\mathrm{~d}, J=6.6 \mathrm{~Hz}, 1 \mathrm{H}), 2.29(\mathrm{~s}, 1 \mathrm{H}), 2.06(\mathrm{~s}, 3 \mathrm{H}),{ }^{13} \mathrm{C} \mathrm{NMR}\left(151 \mathrm{MHz}, \mathrm{CDCl}_{3}\right) \delta$ 147.9, 145.4, $133.1,132.5,128.40,128.38,128.1,127.6,127.2,126.3,126.10,126.09,125.1,123.9,76.5$, 30.9; FTIR (NaCl/thin film) 3560 (brs), 3056, 2978, 2931, 1599, 1505, 1493, 14461, 1372, 1126, $1065,909,858 \mathrm{~cm}^{-1}$; HRMS (EI+) [M]+ calculated for $\mathrm{C}_{18} \mathrm{H}_{16} \mathrm{BO}$ : 248.1201, found: 248.1193 .<smiles>C[C@](O)(c1ccccc1)c1ccc(-c2ccccc2)cc1</smiles>

$(R)$-1-([1,1'-biphenyl]-4-yl)-1-phenylethan-1-ol $((R)-S-1 j)$. Following a similar procedure as described for $(\boldsymbol{R})$-S-1i above, compound $(\boldsymbol{R})$-S-1j was prepared as a white solid (mp 108-111 ${ }^{\circ} \mathrm{C}, 72 \%$ ). The enantiomeric excess was determined to be $91 \%$ by chiral HPLC analysis (CHIRLPAK IB, $1.0 \mathrm{~mL} / \mathrm{min}, 2.0 \% i-\mathrm{PrOH} /$ hexanes, $\lambda=254 \mathrm{~nm}$ ); $\mathrm{t}_{\mathrm{R}}$ (major) $=34.792 \mathrm{~min}$, $\mathrm{t}_{\mathrm{R}}($ minor $)=18.929 \mathrm{~min} .[\alpha]_{\mathrm{D}}{ }^{24}=+9.0^{\circ}\left(\mathrm{c} 1.0, \mathrm{CHCl}_{3}\right):{ }^{1} \mathrm{H} \mathrm{NMR}\left(600 \mathrm{MHz}, \mathrm{CDCl}_{3}\right) \delta 7.61-7.57$ $(\mathrm{m}, 2 \mathrm{H}), 7.57-7.54(\mathrm{~m}, 2 \mathrm{H}), 7.51-7.46(\mathrm{~m}, 4 \mathrm{H}), 7.43(\mathrm{dd}, J=8.4,7.0 \mathrm{~Hz}, 2 \mathrm{H}), 7.38-7.32(\mathrm{~m}$, $3 \mathrm{H}), 7.29-7.26(\mathrm{~m}, 1 \mathrm{H}), 2.22(\mathrm{~s}, 1 \mathrm{H}), 2.00(\mathrm{~s}, 3 \mathrm{H}) ;{ }^{13} \mathrm{C} \mathrm{NMR}\left(151 \mathrm{MHz}, \mathrm{CDCl}_{3}\right) \delta 148.0$, 147.2, 140.9, 140.0, 128.9, 128.4, 127.4, 127.21, 127.17, 127.1, 126.4, 126.0, 76.3, 31.0; FTIR ( NaCl/thin film) 3458 (brs), 3056, 3028, 2978, 1599, 1486, 1449, 1401, 1266, 1171, 1068, 907, $845 \mathrm{~cm}^{-1}$; HRMS (CI+) [M]+H calculated for $\mathrm{C}_{20} \mathrm{H}_{19} \mathrm{O}: 275.1436$, found: 275.1444 . 


\section{Evidence for Stereoretention}

As discussed above, the absolute configurations of $\mathbf{S - 1 d},(S)-\mathbf{1 a}$, and $(R)-\mathbf{1 0}$ were determined by X-ray crystallography. The arylation of $(S)$-1a produced $(R)$-10, demonstrating that this arylation proceeds with overall retention of absolute stereochemistry.
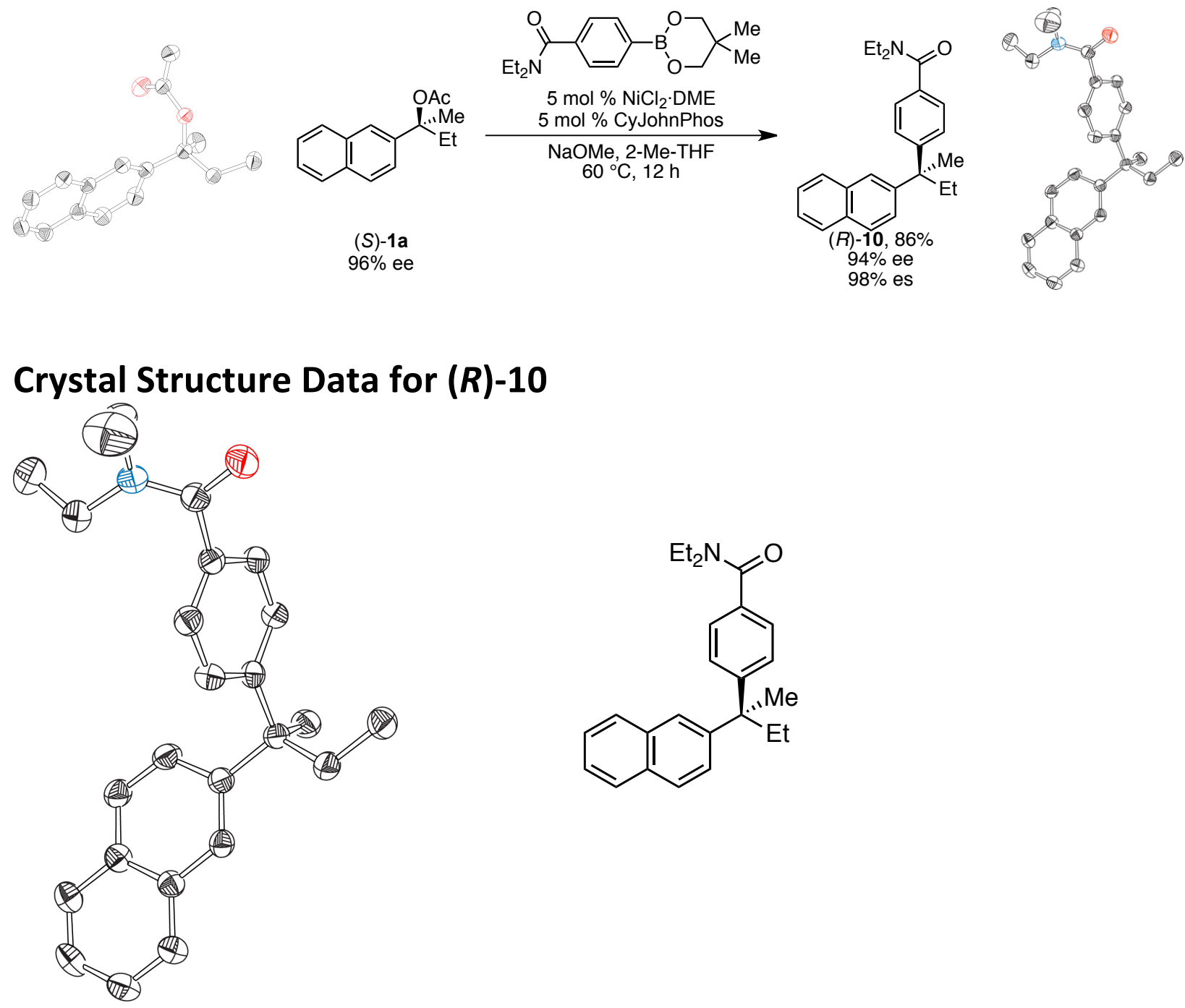

Table S1. Sample and crystal data for $(R)-\mathbf{1 0}$.

Identification code

Chemical formula

Formula weight

Temperature

Wavelength

Crystal size

Crystal system

Space group mary029

$\mathrm{C}_{25} \mathrm{H}_{29} \mathrm{NO}$

359.49

200(2) K

$1.54178 \AA$

$0.248 \times 0.378 \times 0.487 \mathrm{~mm}$

orthorhombic

P 212121 


\begin{tabular}{lll} 
Unit cell dimensions & $\mathrm{a}=8.1727(3) \AA$ & $\alpha=90^{\circ}$ \\
& $\mathrm{b}=12.9877(5) \AA$ & $\beta=90^{\circ}$ \\
& $\mathrm{c}=19.1341(7) \AA$ & $\gamma=90^{\circ}$ \\
Volume & $2030.98(13) \AA^{3}$ & \\
$\mathbf{Z}$ & 4 & \\
Density (calculated) & $1.176 \mathrm{~g} / \mathrm{cm}^{3}$ & \\
Absorption coefficient & $0.540 \mathrm{~mm}^{-1}$ & \\
F(000) & 776 & \\
\hline
\end{tabular}

Table S2. Data collection and structure refinement for $(R)-\mathbf{1 0}$.

Theta range for data collection

Index ranges

Reflections collected

Independent reflections

Coverage of independent reflections

Absorption correction

Max. and min. transmission

Refinement method

Refinement program

Function minimized

Data / restraints / parameters

Goodness-of-fit on $\mathbf{F}^{2}$

Final $\mathbf{R}$ indices

Weighting scheme

Absolute structure parameter

Extinction coefficient

Largest diff. peak and hole

R.M.S. deviation from mean

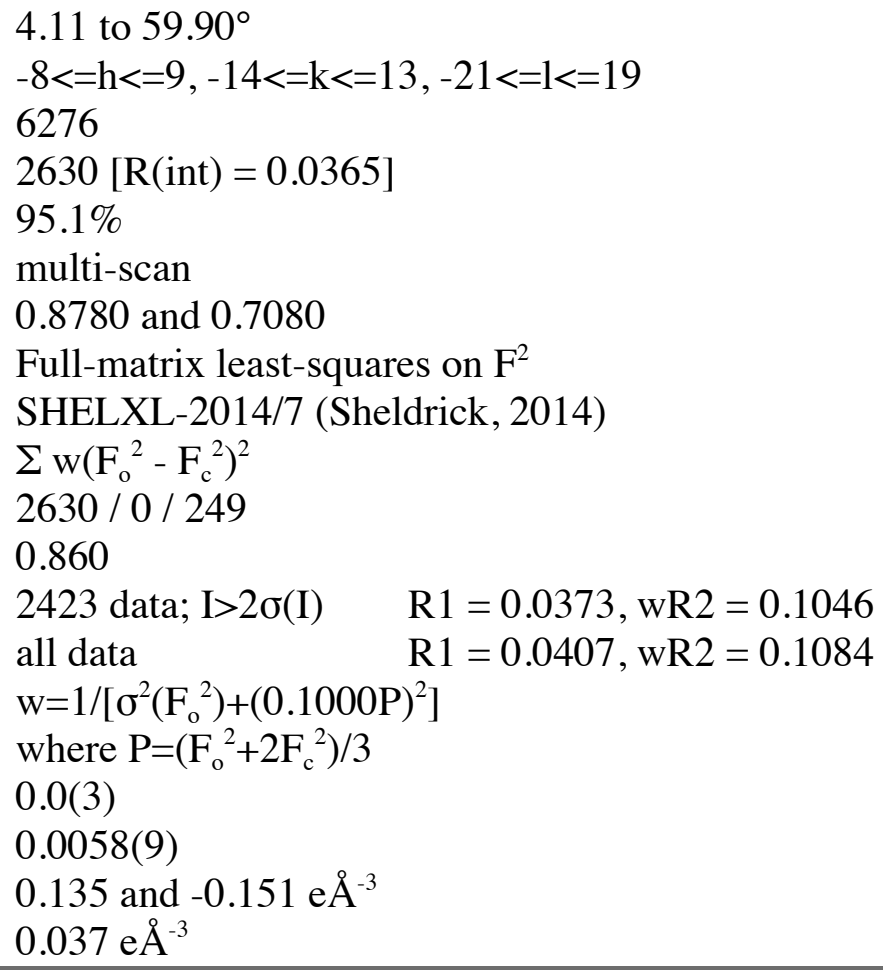

Table S3. Atomic coordinates and equivalent isotropic atomic displacement parameters $\left(\AA^{2}\right)$ for $(R)$ 10.

$\mathrm{U}(\mathrm{eq})$ is defined as one third of the trace of the orthogonalized $\mathrm{U}_{\mathrm{ij}}$ tensor.

\begin{tabular}{lllll}
\hline & $\mathbf{x} / \mathbf{a}$ & $\mathbf{y} / \mathbf{b}$ & $\mathbf{z} / \mathbf{c}$ & $\mathbf{U}(\mathbf{e q})$ \\
$\mathrm{O} 1$ & $0.4981(3)$ & $0.50887(17)$ & $0.01225(10)$ & $0.0528(6)$ \\
$\mathrm{N} 1$ & $0.5202(3)$ & $0.39117(18)$ & $0.09868(13)$ & $0.0412(6)$ \\
$\mathrm{C} 1$ & $0.6431(3)$ & $0.61242(19)$ & $0.24991(13)$ & $0.0288(6)$ \\
$\mathrm{C} 2$ & $0.5755(3)$ & $0.5641(2)$ & $0.30982(14)$ & $0.0311(7)$
\end{tabular}




\begin{tabular}{lllll} 
C3 & $0.4047(4)$ & $0.5453(2)$ & $0.31633(15)$ & $0.0364(7)$ \\
C4 & $0.3434(4)$ & $0.4997(2)$ & $0.37492(16)$ & $0.0445(8)$ \\
C5 & $0.4486(4)$ & $0.4689(2)$ & $0.42913(16)$ & $0.0455(8)$ \\
C6 & $0.6129(4)$ & $0.4850(2)$ & $0.42429(15)$ & $0.0403(7)$ \\
C7 & $0.6800(3)$ & $0.5343(2)$ & $0.36502(14)$ & $0.0334(7)$ \\
C8 & $0.8496(3)$ & $0.5562(2)$ & $0.35893(14)$ & $0.0377(7)$ \\
C9 & $0.9094(3)$ & $0.6044(2)$ & $0.30127(14)$ & $0.0376(8)$ \\
C10 & $0.8070(3)$ & $0.6340(2)$ & $0.24435(13)$ & $0.0305(6)$ \\
C11 & $0.8828(3)$ & $0.6918(2)$ & $0.18274(13)$ & $0.0298(6)$ \\
C12 & $0.9236(4)$ & $0.8013(2)$ & $0.20884(16)$ & $0.0396(7)$ \\
C13 & $0.7628(3)$ & $0.6988(2)$ & $0.12036(13)$ & $0.0354(7)$ \\
C14 & $0.8307(4)$ & $0.7444(2)$ & $0.05339(14)$ & $0.0435(8)$ \\
C15 & $0.0316(3)$ & $0.5282(2)$ & $0.14805(14)$ & $0.0342(7)$ \\
C16 & $0.1636(3)$ & $0.4742(2)$ & $0.12206(14)$ & $0.0355(7)$ \\
C17 & $0.3068(3)$ & $0.5257(2)$ & $0.10319(12)$ & $0.0295(6)$ \\
C18 & $0.3106(3)$ & $0.6315(2)$ & $0.11035(13)$ & $0.0305(6)$ \\
C19 & $0.1794(3)$ & $0.6850(2)$ & $0.13789(13)$ & $0.0303(6)$ \\
C20 & $0.0364(3)$ & $0.6345(2)$ & $0.15721(13)$ & $0.0290(6)$ \\
C21 & $0.4492(4)$ & $0.4735(2)$ & $0.06862(14)$ & $0.0354(7)$ \\
C22 & $0.6428(4)$ & $0.3346(3)$ & $0.05765(18)$ & $0.0536(9)$ \\
C23 & $0.5677(6)$ & $0.2525(3)$ & $0.0140(3)$ & $0.0894(15)$ \\
C24 & $0.4761(4)$ & $0.3501(2)$ & $0.16769(16)$ & $0.0459(8)$ \\
C25 & $0.6199(4)$ & $0.3331(3)$ & $0.21548(18)$ & $0.0530(9)$ \\
\hline
\end{tabular}

Table S4. Bond lengths (A) for $(R)$-10.

$\begin{array}{llll}\mathrm{O} 1-\mathrm{C} 21 & 1.239(3) & \mathrm{N} 1-\mathrm{C} 21 & 1.345(4) \\ \mathrm{N} 1-\mathrm{C} 24 & 1.469(4) & \mathrm{N} 1-\mathrm{C} 22 & 1.470(4) \\ \mathrm{C} 1-\mathrm{C} 10 & 1.373(4) & \mathrm{C} 1-\mathrm{C} 2 & 1.419(4) \\ \mathrm{C} 1-\mathrm{H} 1 & 0.95 & \mathrm{C} 2-\mathrm{C} 7 & 1.413(4) \\ \mathrm{C} 2-\mathrm{C} 3 & 1.422(4) & \mathrm{C} 3-\mathrm{C} 4 & 1.363(4) \\ \mathrm{C} 3-\mathrm{H} 3 & 0.95 & \mathrm{C} 4-\mathrm{C} 5 & 1.406(4) \\ \mathrm{C} 4-\mathrm{H} 4 & 0.95 & \mathrm{C} 5-\mathrm{C} 6 & 1.361(5) \\ \mathrm{C} 5-\mathrm{H} 5 & \mathrm{C} 6-\mathrm{C} 7 & 1.414(4) \\ \mathrm{C} 6-\mathrm{H} 6 & 0.95 & \mathrm{C} 7-\mathrm{C} 8 & 1.419(4) \\ \mathrm{C} 8-\mathrm{C} 9 & \mathrm{C} 8-\mathrm{H} 8 & 0.95 \\ \mathrm{C} 9-\mathrm{C} 10 & 1.359(4) & \mathrm{C} 9-\mathrm{H} 9 & 0.95 \\ \mathrm{C} 10-\mathrm{C} 11 & \mathrm{C} 11-\mathrm{C} 20 & 1.539(4) \\ \mathrm{C} 11-\mathrm{C} 12 & 1.426(4) & \mathrm{C} 11-\mathrm{C} 13 & 1.548(4) \\ \mathrm{C} 12-\mathrm{H} 12 \mathrm{~A} & 1.529(4) & \mathrm{C} 12-\mathrm{H} 12 \mathrm{~B} & 0.98 \\ \mathrm{C} 12-\mathrm{H} 12 \mathrm{C} & 1.543(4) & \mathrm{C} 13-\mathrm{C} 14 & 1.517(4) \\ \mathrm{C} 13-\mathrm{H} 13 \mathrm{~A} & 0.98 & \mathrm{C} 13-\mathrm{H} 13 \mathrm{~B} & 0.99 \\ \mathrm{C} 14-\mathrm{H} 14 \mathrm{~A} & 0.98 & \mathrm{C} 14-\mathrm{H} 14 \mathrm{~B} & 0.98 \\ \mathrm{C} 14-\mathrm{H} 14 \mathrm{C} & 0.99 & \mathrm{C} 15-\mathrm{C} 16 & 1.379(4) \\ \mathrm{C} 15-\mathrm{C} 20 & 0.98 & \mathrm{C} 15-\mathrm{H} 15 & 0.95 \\ \mathrm{C} 16-\mathrm{C} 17 & 0.98 & \mathrm{C} 16-\mathrm{H} 16 & 0.95\end{array}$




\begin{tabular}{llll}
$\mathrm{C} 17-\mathrm{C} 18$ & $1.381(4)$ & $\mathrm{C} 17-\mathrm{C} 21$ & $1.501(4)$ \\
$\mathrm{C} 18-\mathrm{C} 19$ & $1.382(4)$ & $\mathrm{C} 18-\mathrm{H} 18$ & 0.95 \\
$\mathrm{C} 19-\mathrm{C} 20$ & $1.390(4)$ & $\mathrm{C} 19-\mathrm{H} 19$ & 0.95 \\
$\mathrm{C} 22-\mathrm{C} 23$ & $1.487(5)$ & $\mathrm{C} 22-\mathrm{H} 22 \mathrm{~A}$ & 0.99 \\
$\mathrm{C} 22-\mathrm{H} 22 \mathrm{~B}$ & 0.99 & $\mathrm{C} 23-\mathrm{H} 23 \mathrm{~A}$ & 0.98 \\
$\mathrm{C} 23-\mathrm{H} 23 \mathrm{~B}$ & 0.98 & $\mathrm{C} 23-\mathrm{H} 23 \mathrm{C}$ & 0.98 \\
$\mathrm{C} 24-\mathrm{C} 25$ & $1.506(5)$ & $\mathrm{C} 24-\mathrm{H} 24 \mathrm{~A}$ & 0.99 \\
$\mathrm{C} 24-\mathrm{H} 24 \mathrm{~B}$ & 0.99 & $\mathrm{C} 25-\mathrm{H} 25 \mathrm{~A}$ & 0.98 \\
$\mathrm{C} 25-\mathrm{H} 25 \mathrm{~B}$ & 0.98 & $\mathrm{C} 25-\mathrm{H} 25 \mathrm{C}$ & 0.98 \\
\hline
\end{tabular}

Table S5. Bond angles $\left(^{\circ}\right)$ for $(R)-\mathbf{1 0}$.

\begin{tabular}{|c|c|c|c|}
\hline C21-N1-C24 & $124.5(2)$ & C21-N1-C22 & $117.6(2)$ \\
\hline C24-N1-C22 & $117.8(2)$ & C10-C1-C2 & $122.2(2)$ \\
\hline C10-C1-H1 & 118.9 & $\mathrm{C} 2-\mathrm{C} 1-\mathrm{H} 1$ & 118.9 \\
\hline $\mathrm{C} 7-\mathrm{C} 2-\mathrm{C} 1$ & $119.3(2)$ & $\mathrm{C} 7-\mathrm{C} 2-\mathrm{C} 3$ & $118.8(3)$ \\
\hline $\mathrm{C} 1-\mathrm{C} 2-\mathrm{C} 3$ & $121.9(3)$ & $\mathrm{C} 4-\mathrm{C} 3-\mathrm{C} 2$ & $120.5(3)$ \\
\hline C4-C3-H3 & 119.8 & $\mathrm{C} 2-\mathrm{C} 3-\mathrm{H} 3$ & 119.8 \\
\hline $\mathrm{C} 3-\mathrm{C} 4-\mathrm{C} 5$ & $120.4(3)$ & $\mathrm{C} 3-\mathrm{C} 4-\mathrm{H} 4$ & 119.8 \\
\hline C5-C4-H4 & 119.8 & $\mathrm{C} 6-\mathrm{C} 5-\mathrm{C} 4$ & $120.6(3)$ \\
\hline C6-C5-H5 & 119.7 & C4-C5-H5 & 119.7 \\
\hline C5-C6-C7 & $120.5(3)$ & C5-C6-H6 & 119.8 \\
\hline C7-C6-H6 & 119.8 & C2-C7-C6 & $119.3(3)$ \\
\hline $\mathrm{C} 2-\mathrm{C} 7-\mathrm{C} 8$ & $118.3(2)$ & C6-C7-C8 & $122.4(3)$ \\
\hline C9-C8-C7 & $120.7(3)$ & C9-C8-H8 & 119.7 \\
\hline C7-C8-H8 & 119.7 & C8-C9-C10 & $122.2(3)$ \\
\hline C8-C9-H9 & 118.9 & C10-C9-H9 & 118.9 \\
\hline C1-C10-C9 & $117.3(2)$ & C1-C10-C11 & $123.7(2)$ \\
\hline C9-C10-C11 & $118.9(2)$ & C10-C11-C20 & $109.7(2)$ \\
\hline C10-C11-C12 & $106.9(2)$ & C20-C11-C12 & $111.8(2)$ \\
\hline C10-C11-C13 & $111.5(2)$ & C20-C11-C13 & $107.5(2)$ \\
\hline C12-C11-C13 & $109.4(2)$ & C11-C12-H12A & 109.5 \\
\hline C11-C12-H12B & 109.5 & $\mathrm{H} 12 \mathrm{~A}-\mathrm{C} 12-\mathrm{H} 12 \mathrm{~B}$ & 109.5 \\
\hline C11-C12-H12C & 109.5 & $\mathrm{H} 12 \mathrm{~A}-\mathrm{C} 12-\mathrm{H} 12 \mathrm{C}$ & 109.5 \\
\hline $\mathrm{H} 12 \mathrm{~B}-\mathrm{C} 12-\mathrm{H} 12 \mathrm{C}$ & 109.5 & C14-C13-C11 & $116.3(2)$ \\
\hline C14-C13-H13A & 108.2 & C11-C13-H13A & 108.2 \\
\hline C14-C13-H13B & 108.2 & C11-C13-H13B & 108.2 \\
\hline H13A-C13-H13B & 107.4 & C13-C14-H14A & 109.5 \\
\hline C13-C14-H14B & 109.5 & H14A-C14-H14B & 109.5 \\
\hline C13-C14-H14C & 109.5 & H14A-C14-H14C & 109.5 \\
\hline H14B-C14-H14C & 109.5 & C16-C15-C20 & $121.8(3)$ \\
\hline C16-C15-H15 & 119.1 & C20-C15-H15 & 119.1 \\
\hline C15-C16-C17 & $120.4(3)$ & C15-C16-H16 & 119.8 \\
\hline C17-C16-H16 & 119.8 & C18-C17-C16 & $118.1(2)$ \\
\hline C18-C17-C21 & $118.4(2)$ & C16-C17-C21 & $123.2(2)$ \\
\hline C17-C18-C19 & $121.4(3)$ & C17-C18-H18 & 119.3 \\
\hline
\end{tabular}




\begin{tabular}{llll}
$\mathrm{C} 19-\mathrm{C} 18-\mathrm{H} 18$ & 119.3 & $\mathrm{C} 18-\mathrm{C} 19-\mathrm{C} 20$ & $121.1(2)$ \\
$\mathrm{C} 18-\mathrm{C} 19-\mathrm{H} 19$ & 119.5 & $\mathrm{C} 20-\mathrm{C} 19-\mathrm{H} 19$ & 119.5 \\
$\mathrm{C} 19-\mathrm{C} 20-\mathrm{C} 15$ & $117.3(2)$ & $\mathrm{C} 19-\mathrm{C} 20-\mathrm{C} 11$ & $122.8(2)$ \\
$\mathrm{C} 15-\mathrm{C} 20-\mathrm{C} 11$ & $119.8(2)$ & $\mathrm{O} 1-\mathrm{C} 21-\mathrm{N} 1$ & $121.9(3)$ \\
$\mathrm{O} 1-\mathrm{C} 21-\mathrm{C} 17$ & $117.8(3)$ & $\mathrm{N} 1-\mathrm{C} 21-\mathrm{C} 17$ & $120.4(2)$ \\
$\mathrm{N} 1-\mathrm{C} 22-\mathrm{C} 23$ & $112.1(3)$ & $\mathrm{N} 1-\mathrm{C} 22-\mathrm{H} 22 \mathrm{~A}$ & 109.2 \\
$\mathrm{C} 23-\mathrm{C} 22-\mathrm{H} 22 \mathrm{~A}$ & 109.2 & $\mathrm{~N} 1-\mathrm{C} 22-\mathrm{H} 22 \mathrm{~B}$ & 109.2 \\
$\mathrm{C} 23-\mathrm{C} 22-\mathrm{H} 22 \mathrm{~B}$ & 109.2 & $\mathrm{H} 22 \mathrm{~A}-\mathrm{C} 22-\mathrm{H} 22 \mathrm{~B}$ & 107.9 \\
$\mathrm{C} 22-\mathrm{C} 23-\mathrm{H} 23 \mathrm{~A}$ & 109.5 & $\mathrm{C} 22-\mathrm{C} 23-\mathrm{H} 23 \mathrm{~B}$ & 109.5 \\
$\mathrm{H} 23 \mathrm{~A}-\mathrm{C} 23-\mathrm{H} 23 \mathrm{~B}$ & 109.5 & $\mathrm{C} 22-\mathrm{C} 23-\mathrm{H} 23 \mathrm{C}$ & 109.5 \\
$\mathrm{H} 23 \mathrm{~A}-\mathrm{C} 23-\mathrm{H} 23 \mathrm{C}$ & 109.5 & $\mathrm{H} 23 \mathrm{~B}-\mathrm{C} 23-\mathrm{H} 23 \mathrm{C}$ & 109.5 \\
$\mathrm{~N} 1-\mathrm{C} 24-\mathrm{C} 25$ & $114.1(3)$ & $\mathrm{N} 1-\mathrm{C} 24-\mathrm{H} 24 \mathrm{~A}$ & 108.7 \\
$\mathrm{C} 25-\mathrm{C} 24-\mathrm{H} 24 \mathrm{~A}$ & 108.7 & $\mathrm{~N} 1-\mathrm{C} 24-\mathrm{H} 24 \mathrm{~B}$ & 108.7 \\
$\mathrm{C} 25-\mathrm{C} 24-\mathrm{H} 24 \mathrm{~B}$ & 108.7 & $\mathrm{H} 24 \mathrm{~A}-\mathrm{C} 24-\mathrm{H} 24 \mathrm{~B}$ & 107.6 \\
$\mathrm{C} 24-\mathrm{C} 25-\mathrm{H} 25 \mathrm{~A}$ & 109.5 & $\mathrm{C} 24-\mathrm{C} 25-\mathrm{H} 25 \mathrm{~B}$ & 109.5 \\
$\mathrm{H} 25 \mathrm{~A}-\mathrm{C} 25-\mathrm{H} 25 \mathrm{~B}$ & 109.5 & $\mathrm{C} 24-\mathrm{C} 25-\mathrm{H} 25 \mathrm{C}$ & 109.5 \\
$\mathrm{H} 25 \mathrm{~A}-\mathrm{C} 25-\mathrm{H} 25 \mathrm{C}$ & 109.5 & $\mathrm{H} 25 \mathrm{~B}-\mathrm{C} 25-\mathrm{H} 25 \mathrm{C}$ & 109.5 \\
\hline
\end{tabular}

Table S6. Torsion angles $\left({ }^{\circ}\right)$ for $(R)-\mathbf{1 0}$.

$\begin{array}{llll}\text { C10-C1-C2-C7 } & -1.8(4) & \text { C10-C1-C2-C3 } & 177.7(3) \\ \text { C7-C2-C3-C4 } & 0.3(4) & \text { C1-C2-C3-C4 } & -179.2(3) \\ \text { C2-C3-C4-C5 } & -1.3(4) & \text { C3-C4-C5-C6 } & 0.7(5) \\ \text { C4-C5-C6-C7 } & 0.9(5) & \text { C1-C2-C7-C6 } & -179.2(2) \\ \text { C3-C2-C7-C6 } & 1.3(4) & \text { C1-C2-C7-C8 } & 1.4(4) \\ \text { C3-C2-C7-C8 } & -178.2(3) & \text { C5-C6-C7-C2 } & -1.9(4) \\ \text { C5-C6-C7-C8 } & 177.5(3) & \text { C2-C7-C8-C9 } & -0.2(4) \\ \text { C6-C7-C8-C9 } & -179.6(3) & \text { C7-C8-C9-C10 } & -0.7(5) \\ \text { C2-C1-C10-C9 } & 1.0(4) & \text { C2-C1-C10-C11 } & -176.1(2) \\ \text { C8-C9-C10-C1 } & 0.3(4) & \text { C8-C9-C10-C11 } & 177.5(3) \\ \text { C1-C10-C11-C20 } & -133.0(3) & \text { C9-C10-C11-C20 } & 50.0(3) \\ \text { C1-C10-C11-C12 } & 105.5(3) & \text { C9-C10-C11-C12 } & -71.5(3) \\ \text { C1-C10-C11-C13 } & -14.1(4) & \text { C9-C10-C11-C13 } & 168.9(2) \\ \text { C10-C11-C13-C14 } & -173.7(2) & \text { C20-C11-C13-C14 } & -53.4(3) \\ \text { C12-C11-C13-C14 } & 68.3(3) & \text { C20-C15-C16-C17 } & -0.8(4) \\ \text { C15-C16-C17-C18 } & -1.0(4) & \text { C15-C16-C17-C21 } & -174.3(2) \\ \text { C16-C17-C18-C19 } & 2.5(4) & \text { C21-C17-C18-C19 } & 176.1(2) \\ \text { C17-C18-C19-C20 } & -2.2(4) & \text { C18-C19-C20-C15 } & 0.4(4) \\ \text { C18-C19-C20-C11 } & -175.2(2) & \text { C16-C15-C20-C19 } & 1.1(4) \\ \text { C16-C15-C20-C11 } & 176.8(2) & \text { C10-C11-C20-C19 } & -138.7(3) \\ \text { C12-C11-C20-C19 } & -20.3(4) & \text { C13-C11-C20-C19 } & 99.9(3) \\ \text { C10-C11-C20-C15 } & 45.9(3) & \text { C12-C11-C20-C15 } & 164.3(2) \\ \text { C13-C11-C20-C15 } & -75.6(3) & \text { C24-N1-C21-O1 } & 175.3(3) \\ \text { C22-N1-C21-O1 } & -8.8(4) & \text { C24-N1-C21-C17 } & -4.5(4) \\ \text { C22-N1-C21-C17 } & 171.3(3) & \text { C18-C17-C21-O1 } & -48.9(4) \\ \end{array}$




\begin{tabular}{llll}
$\mathrm{C} 16-\mathrm{C} 17-\mathrm{C} 21-\mathrm{O} 1$ & $124.4(3)$ & $\mathrm{C} 18-\mathrm{C} 17-\mathrm{C} 21-\mathrm{N} 1$ & $131.0(3)$ \\
$\mathrm{C} 16-\mathrm{C} 17-\mathrm{C} 21-\mathrm{N} 1$ & $-55.7(4)$ & $\mathrm{C} 21-\mathrm{N} 1-\mathrm{C} 22-\mathrm{C} 23$ & $-88.4(4)$ \\
$\mathrm{C} 24-\mathrm{N} 1-\mathrm{C} 22-\mathrm{C} 23$ & $87.7(4)$ & $\mathrm{C} 21-\mathrm{N} 1-\mathrm{C} 24-\mathrm{C} 25$ & $-129.8(3)$ \\
$\mathrm{C} 22-\mathrm{N} 1-\mathrm{C} 24-\mathrm{C} 25$ & $54.3(4)$ & & \\
\hline
\end{tabular}

Table S7. Anisotropic atomic displacement parameters $\left(\AA^{2}\right)$ for $(R)-\mathbf{1 0}$.

The anisotropic atomic displacement factor exponent takes the form: $-2 \pi^{2}\left[\mathrm{~h}^{2} \mathrm{a}^{* 2} \mathrm{U}_{11}+\ldots+2 \mathrm{~h} \mathrm{k} \mathrm{a}^{*} \mathrm{~b}^{*}\right.$ $\left.\mathrm{U}_{12}\right]$

\begin{tabular}{lllllll} 
& $\mathbf{U}_{11}$ & $\mathbf{U}_{22}$ & $\mathbf{U}_{33}$ & $\mathbf{U}_{23}$ & $\mathbf{U}_{13}$ & $\mathbf{U}_{12}$ \\
$\mathrm{O} 1$ & $0.0573(14)$ & $0.0621(14)$ & $0.0391(12)$ & $0.0079(11)$ & $0.0201(12)$ & $0.0140(12)$ \\
$\mathrm{N} 1$ & $0.0358(14)$ & $0.0424(14)$ & $0.0453(14)$ & $0.0030(12)$ & $0.0100(12)$ & $0.0097(12)$ \\
$\mathrm{C} 1$ & $0.0289(14)$ & $0.0312(14)$ & $0.0262(14)$ & $0.0003(12)$ & $-0.0029(12)$ & $0.0033(11)$ \\
$\mathrm{C} 2$ & $0.0331(15)$ & $0.0278(15)$ & $0.0323(16)$ & $-0.0029(12)$ & $0.0014(13)$ & $-0.0010(12)$ \\
$\mathrm{C} 3$ & $0.0313(15)$ & $0.0395(17)$ & $0.0384(17)$ & $0.0019(14)$ & $0.0009(14)$ & $-0.0025(13)$ \\
$\mathrm{C} 4$ & $0.0386(17)$ & $0.0449(18)$ & $0.0499(19)$ & $0.0010(16)$ & $0.0045(15)$ & $-0.0121(14)$ \\
$\mathrm{C} 5$ & $0.0537(19)$ & $0.0443(18)$ & $0.0385(18)$ & $0.0054(14)$ & $0.0095(15)$ & $-0.0116(15)$ \\
$\mathrm{C} 6$ & $0.0464(18)$ & $0.0413(17)$ & $0.0332(16)$ & $0.0040(13)$ & $0.0004(15)$ & $-0.0025(14)$ \\
$\mathrm{C} 7$ & $0.0356(15)$ & $0.0325(15)$ & $0.0319(15)$ & $-0.0034(13)$ & $-0.0005(13)$ & $0.0001(12)$ \\
$\mathrm{C} 8$ & $0.0315(15)$ & $0.0515(18)$ & $0.0301(15)$ & $0.0065(14)$ & $-0.0023(14)$ & $0.0056(13)$ \\
$\mathrm{C} 9$ & $0.0252(15)$ & $0.0516(19)$ & $0.0360(17)$ & $0.0004(14)$ & $-0.0015(13)$ & $0.0014(13)$ \\
$\mathrm{C} 10$ & $0.0276(13)$ & $0.0337(15)$ & $0.0302(14)$ & $0.0024(12)$ & $0.0000(13)$ & $0.0047(12)$ \\
$\mathrm{C} 11$ & $0.0230(13)$ & $0.0367(15)$ & $0.0295(14)$ & $0.0017(12)$ & $0.0004(12)$ & $0.0030(11)$ \\
$\mathrm{C} 12$ & $0.0370(16)$ & $0.0366(16)$ & $0.0453(17)$ & $-0.0011(14)$ & $0.0076(14)$ & $0.0029(13)$ \\
$\mathrm{C} 13$ & $0.0297(15)$ & $0.0412(16)$ & $0.0353(16)$ & $0.0038(13)$ & $0.0005(12)$ & $0.0052(13)$ \\
$\mathrm{C} 14$ & $0.0436(19)$ & $0.0510(17)$ & $0.0359(15)$ & $0.0077(15)$ & $0.0006(15)$ & $0.0077(15)$ \\
$\mathrm{C} 15$ & $0.0274(14)$ & $0.0372(17)$ & $0.0379(15)$ & $0.0044(13)$ & $0.0035(13)$ & $-0.0020(12)$ \\
$\mathrm{C} 16$ & $0.0367(16)$ & $0.0305(15)$ & $0.0392(16)$ & $-0.0013(13)$ & $0.0041(13)$ & $0.0014(13)$ \\
$\mathrm{C} 17$ & $0.0300(14)$ & $0.0378(16)$ & $0.0207(13)$ & $0.0026(12)$ & $0.0005(12)$ & $0.0046(13)$ \\
$\mathrm{C} 18$ & $0.0255(13)$ & $0.0392(16)$ & $0.0269(13)$ & $0.0054(12)$ & $-0.0017(12)$ & $-0.0016(12)$ \\
$\mathrm{C} 19$ & $0.0290(15)$ & $0.0312(14)$ & $0.0309(14)$ & $0.0011(12)$ & $0.0013(13)$ & $0.0009(12)$ \\
$\mathrm{C} 20$ & $0.0278(14)$ & $0.0329(16)$ & $0.0262(14)$ & $0.0039(12)$ & $-0.0015(12)$ & $0.0009(11)$ \\
$\mathrm{C} 21$ & $0.0330(15)$ & $0.0413(17)$ & $0.0319(16)$ & $-0.0020(13)$ & $0.0045(13)$ & $0.0039(13)$ \\
$\mathrm{C} 22$ & $0.0482(19)$ & $0.0486(19)$ & $0.064(2)$ & $-0.0055(18)$ & $0.0122(18)$ & $0.0142(16)$ \\
$\mathrm{C} 23$ & $0.092(3)$ & $0.063(2)$ & $0.113(4)$ & $-0.035(3)$ & $0.027(3)$ & $-0.003(2)$ \\
$\mathrm{C} 24$ & $0.0411(17)$ & $0.0452(18)$ & $0.0513(18)$ & $0.0145(15)$ & $0.0062(16)$ & $0.0087(14)$ \\
$\mathrm{C} 25$ & $0.051(2)$ & $0.0470(19)$ & $0.061(2)$ & $0.0045(16)$ & $-0.0059(18)$ & $0.0073(16)$ \\
\hline & & & & & &
\end{tabular}

Table S8. Hydrogen atomic coordinates and isotropic atomic displacement parameters $\left(\AA^{2}\right)$ for $(R)$ 10.

$\begin{array}{lllll} & \mathbf{x} / \mathbf{a} & \mathbf{y} / \mathbf{b} & \mathbf{z} / \mathbf{c} & \mathbf{U}(\mathbf{e q}) \\ \mathrm{H} 1 & -0.4274 & 0.6304 & 0.2124 & 0.035 \\ \mathrm{H} 3 & -0.6672 & 0.5646 & 0.2796 & 0.044\end{array}$




\begin{tabular}{lllll} 
H4 & -0.7711 & 0.4887 & 0.3792 & 0.053 \\
H5 & -0.5952 & 0.4366 & 0.4695 & 0.055 \\
H6 & -0.3172 & 0.4629 & 0.4610 & 0.048 \\
H8 & -0.0780 & 0.5370 & 0.3955 & 0.045 \\
H9 & 0.0232 & 0.6188 & 0.2988 & 0.045 \\
H12A & -0.1767 & 0.8350 & 0.2251 & 0.059 \\
H12B & -0.0286 & 0.8413 & 0.1705 & 0.059 \\
H12C & 0.0021 & 0.7970 & 0.2475 & 0.059 \\
H13A & -0.2777 & 0.6285 & 0.1100 & 0.042 \\
H13B & -0.3326 & 0.7405 & 0.1350 & 0.042 \\
H14A & -0.1280 & 0.8139 & 0.0626 & 0.065 \\
H14B & -0.2562 & 0.7477 & 0.0182 & 0.065 \\
H14C & -0.0799 & 0.7010 & 0.0361 & 0.065 \\
H15 & -0.0653 & 0.4918 & 0.1600 & 0.041 \\
H16 & 0.1570 & 0.4016 & 0.1170 & 0.043 \\
H18 & 0.4054 & 0.6682 & 0.0960 & 0.037 \\
H19 & 0.1871 & 0.7575 & 0.1437 & 0.036 \\
H22A & 0.7236 & 0.3032 & 0.0898 & 0.064 \\
H22B & 0.7019 & 0.3834 & 0.0270 & 0.064 \\
H23A & 0.5062 & 0.2051 & 0.0440 & 0.134 \\
H23B & 0.6538 & 0.2145 & -0.0105 & 0.134 \\
H23C & 0.4934 & 0.2837 & -0.0201 & 0.134 \\
H24A & 0.4182 & 0.2838 & 0.1613 & 0.055 \\
H24B & 0.3992 & 0.3985 & 0.1905 & 0.055 \\
H25A & 0.6849 & 0.2750 & 0.1984 & 0.079 \\
H25B & 0.5805 & 0.3181 & 0.2628 & 0.079 \\
H25C & 0.6879 & 0.3953 & 0.2164 & \\
\hline
\end{tabular}




\section{Crystal Structure Data for 1a}

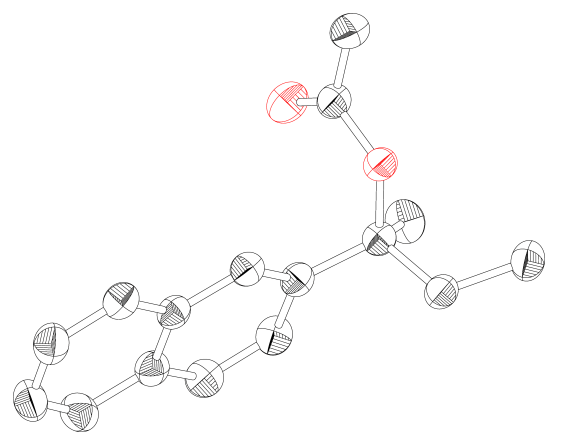<smiles>CCC(C)(OC(C)=O)c1ccc2ccccc2c1</smiles>

Table S9. Sample and crystal data for $(S)-1$ a.

Identification

code

mary035

Chemical

formula

$\mathrm{C}_{16} \mathrm{H}_{18} \mathrm{O}_{2}$

Formula weight

$242.30 \mathrm{~g} / \mathrm{mol}$

Temperature

200(2) K

Wavelength

$1.54178 \AA$

Crystal size

$0.144 \times 0.196 \times 0.269 \mathrm{~mm}$

Crystal system

monoclinic

Space group

C 121

Unit cell

dimensions

$\mathrm{a}=21.7695(7) \AA$

$\alpha=90^{\circ}$

$\mathrm{b}=5.8807(2) \AA$

$\mathrm{c}=10.4637(3) \AA$

Volume

$1328.76(7) \AA^{3}$

Z

4

Density

(calculated)

$1.211 \mathrm{~g} / \mathrm{cm}^{3}$

Absorption

coefficient

$0.620 \mathrm{~mm}^{-1}$

$\mathbf{F}(000)$

520

Table S10. Data collection and structure refinement for $(S)-1$ a.

Theta range for data collection

4.09 to $75.07^{\circ}$

Index ranges

Reflections collected

$-27<=\mathrm{h}<=26,-7<=\mathrm{k}<=7,-13<=\mathrm{l}<=12$

Independent reflections

13728

Coverage of independent reflections

$2708[\mathrm{R}(\mathrm{int})=0.0319]$

Absorption correction

$99.4 \%$

Max. and min. transmission

multi-scan

Structure solution technique

0.7539 and 0.6441

Structure solution program

direct methods

$\beta=97.282(2)^{\circ}$

$\gamma=90^{\circ}$

SHELXS-97 (Sheldrick 2008) 
Refinement method

Refinement program

Function minimized

Data / restraints / parameters

Goodness-of-fit on $\mathbf{F}^{2}$

Final $\mathbf{R}$ indices

Weighting scheme

Absolute structure parameter

Largest diff. peak and hole

R.M.S. deviation from mean
Full-matrix least-squares on $\mathrm{F}^{2}$

SHELXL-2014/7 (Sheldrick, 2014)

$\Sigma \mathrm{w}\left(\mathrm{F}_{\mathrm{o}}^{2}-\mathrm{F}_{\mathrm{c}}^{2}\right)^{2}$

$2708 / 1 / 166$

1.035

2626 data; $\mathrm{I}>2 \sigma(\mathrm{I}) \quad \mathrm{R} 1=0.0349, \mathrm{wR} 2=0.0937$

all data $\quad \mathrm{R} 1=0.0359, \mathrm{wR} 2=0.0948$

$\mathrm{W}=1 /\left[\sigma^{2}\left(\mathrm{~F}_{\mathrm{o}}^{2}\right)+(0.0638 \mathrm{P})^{2}+0.1971 \mathrm{P}\right]$

where $\mathrm{P}=\left(\mathrm{F}_{\mathrm{o}}^{2}+2 \mathrm{~F}_{\mathrm{c}}^{2}\right) / 3$

$-0.1(1)$

0.193 and $-0.164 \mathrm{e}^{-3}$

$0.034 \mathrm{e}^{-3}$

Table S11. Atomic coordinates and equivalent isotropic atomic displacement parameters $\left(\breve{\mathrm{A}}^{2}\right)$ for $(S)$-1a.

$\mathrm{U}(\mathrm{eq})$ is defined as one third of the trace of the orthogonalized $\mathrm{U}_{\mathrm{ij}}$ tensor.

\begin{tabular}{lllll} 
& $\mathbf{x} / \mathbf{a}$ & $\mathbf{y} / \mathbf{b}$ & $\mathbf{z} / \mathbf{c}$ & $\mathbf{U}(\mathbf{e q})$ \\
O1 & $0.40605(5)$ & $0.6779(2)$ & $0.16441(11)$ & $0.0352(3)$ \\
O2 & $0.32500(7)$ & $0.4448(3)$ & $0.10507(15)$ & $0.0560(4)$ \\
C1 & $0.44069(7)$ & $0.5057(3)$ & $0.24545(16)$ & $0.0324(4)$ \\
C2 & $0.45441(10)$ & $0.3005(3)$ & $0.1639(2)$ & $0.0452(4)$ \\
C3 & $0.50055(7)$ & $0.6315(3)$ & $0.29722(16)$ & $0.0353(4)$ \\
C4 & $0.54151(8)$ & $0.6990(4)$ & $0.19539(19)$ & $0.0444(4)$ \\
C5 & $0.35048(8)$ & $0.6252(4)$ & $0.09814(17)$ & $0.0407(4)$ \\
C6 & $0.32655(9)$ & $0.8226(5)$ & $0.0164(2)$ & $0.0528(5)$ \\
C7 & $0.40667(7)$ & $0.4437(3)$ & $0.35920(16)$ & $0.0324(4)$ \\
C8 & $0.42021(8)$ & $0.2325(3)$ & $0.42318(19)$ & $0.0378(4)$ \\
C9 & $0.39467(8)$ & $0.1768(3)$ & $0.53140(18)$ & $0.0398(4)$ \\
C10 & $0.35368(8)$ & $0.3262(3)$ & $0.58396(18)$ & $0.0358(4)$ \\
C11 & $0.32657(9)$ & $0.2747(4)$ & $0.69691(19)$ & $0.0445(4)$ \\
C12 & $0.28692(9)$ & $0.4252(4)$ & $0.74387(19)$ & $0.0481(5)$ \\
C13 & $0.27174(9)$ & $0.6322(4)$ & $0.67982(19)$ & $0.0453(5)$ \\
C14 & $0.29716(8)$ & $0.6866(3)$ & $0.57105(18)$ & $0.0381(4)$ \\
C15 & $0.33914(7)$ & $0.5370(3)$ & $0.52055(17)$ & $0.0325(4)$ \\
C16 & $0.36692(7)$ & $0.5906(3)$ & $0.40857(16)$ & $0.0327(3)$ \\
\hline
\end{tabular}

Table S12. Bond lengths (Å) for $(S)-1$.

$\begin{array}{llll}\text { O1-C5 } & 1.352(2) & \text { O1-C1 } & 1.466(2) \\ \text { O2-C5 } & 1.204(3) & \text { C1-C7 } & 1.524(2) \\ \text { C1-C2 } & 1.529(2) & \text { C1-C3 } & 1.536(2) \\ \text { C2-H2A } & 0.98 & \text { C2-H2B } & 0.98 \\ \text { C2-H2C } & 0.98 & \text { C3-C4 } & 1.526(2) \\ \text { C3-H3A } & 0.99 & \text { C3-H3B } & 0.99 \\ \text { C4-H4A } & 0.98 & \text { C4-H4B } & 0.98\end{array}$




\begin{tabular}{llll} 
C4-H4C & 0.98 & C5-C6 & $1.496(3)$ \\
C6-H6A & 0.98 & C6-H6B & 0.98 \\
C6-H6C & 0.98 & C7-C16 & $1.369(2)$ \\
C7-C8 & $1.424(2)$ & C8-C9 & $1.363(3)$ \\
C8-H8 & 0.95 & C9-C10 & $1.413(3)$ \\
C9-H9 & 0.95 & C10-C11 & $1.419(3)$ \\
C10-C15 & $1.423(2)$ & C11-C12 & $1.370(3)$ \\
C11-H11 & 0.95 & C12-C13 & $1.409(3)$ \\
C12-H12 & 0.95 & C13-C14 & $1.365(3)$ \\
C13-H13 & 0.95 & C14-C15 & $1.418(2)$ \\
C14-H14 & 0.95 & C15-C16 & $1.420(2)$ \\
C16-H16 & 0.95 & & \\
\hline
\end{tabular}

Table S13. Bond angles $\left({ }^{\circ}\right)$ for $(S)$-1a.

\begin{tabular}{|c|c|c|c|}
\hline $\mathrm{C} 5-\mathrm{O} 1-\mathrm{C} 1$ & $119.98(14)$ & $\mathrm{O} 1-\mathrm{C} 1-\mathrm{C} 7$ & $110.59(12)$ \\
\hline $\mathrm{O} 1-\mathrm{C} 1-\mathrm{C} 2$ & $110.15(14)$ & $\mathrm{C} 7-\mathrm{C} 1-\mathrm{C} 2$ & $113.31(15)$ \\
\hline $\mathrm{O} 1-\mathrm{C} 1-\mathrm{C} 3$ & $102.86(13)$ & $\mathrm{C} 7-\mathrm{C} 1-\mathrm{C} 3$ & $108.33(13)$ \\
\hline $\mathrm{C} 2-\mathrm{C} 1-\mathrm{C} 3$ & $111.09(14)$ & $\mathrm{C} 1-\mathrm{C} 2-\mathrm{H} 2 \mathrm{~A}$ & 109.5 \\
\hline $\mathrm{C} 1-\mathrm{C} 2-\mathrm{H} 2 \mathrm{~B}$ & 109.5 & $\mathrm{H} 2 \mathrm{~A}-\mathrm{C} 2-\mathrm{H} 2 \mathrm{~B}$ & 109.5 \\
\hline $\mathrm{C} 1-\mathrm{C} 2-\mathrm{H} 2 \mathrm{C}$ & 109.5 & $\mathrm{H} 2 \mathrm{~A}-\mathrm{C} 2-\mathrm{H} 2 \mathrm{C}$ & 109.5 \\
\hline $\mathrm{H} 2 \mathrm{~B}-\mathrm{C} 2-\mathrm{H} 2 \mathrm{C}$ & 109.5 & $\mathrm{C} 4-\mathrm{C} 3-\mathrm{C} 1$ & $115.06(14)$ \\
\hline C4-C3-H3A & 108.5 & C1-C3-H3A & 108.5 \\
\hline C4-C3-H3B & 108.5 & C1-C3-H3B & 108.5 \\
\hline $\mathrm{H} 3 \mathrm{~A}-\mathrm{C} 3-\mathrm{H} 3 \mathrm{~B}$ & 107.5 & $\mathrm{C} 3-\mathrm{C} 4-\mathrm{H} 4 \mathrm{~A}$ & 109.5 \\
\hline C3-C4-H4B & 109.5 & H4A-C4-H4B & 109.5 \\
\hline C3-C4-H4C & 109.5 & $\mathrm{H} 4 \mathrm{~A}-\mathrm{C} 4-\mathrm{H} 4 \mathrm{C}$ & 109.5 \\
\hline $\mathrm{H} 4 \mathrm{~B}-\mathrm{C} 4-\mathrm{H} 4 \mathrm{C}$ & 109.5 & $\mathrm{O} 2-\mathrm{C} 5-\mathrm{O} 1$ & $124.07(19)$ \\
\hline $\mathrm{O} 2-\mathrm{C} 5-\mathrm{C} 6$ & $126.06(18)$ & $\mathrm{O} 1-\mathrm{C} 5-\mathrm{C} 6$ & $109.87(17)$ \\
\hline C5-C6-H6A & 109.5 & C5-C6-H6B & 109.5 \\
\hline H6A-C6-H6B & 109.5 & C5-C6-H6C & 109.5 \\
\hline H6A-C6-H6C & 109.5 & H6B-C6-H6C & 109.5 \\
\hline C16-C7-C8 & $118.41(16)$ & C16-C7-C1 & $122.46(15)$ \\
\hline $\mathrm{C} 8-\mathrm{C} 7-\mathrm{C} 1$ & $118.95(15)$ & C9-C8-C7 & $121.23(17)$ \\
\hline C9-C8-H8 & 119.4 & C7-C8-H8 & 119.4 \\
\hline C8-C9-C10 & $121.20(17)$ & C8-C9-H9 & 119.4 \\
\hline C10-C9-H9 & 119.4 & C9-C10-C11 & $122.78(17)$ \\
\hline C9-C10-C15 & $118.34(16)$ & C11-C10-C15 & $118.88(16)$ \\
\hline C12-C11-C10 & $120.54(19)$ & C12-C11-H11 & 119.7 \\
\hline C10-C11-H11 & 119.7 & C11-C12-C13 & $120.54(18)$ \\
\hline C11-C12-H12 & 119.7 & C13-C12-H12 & 119.7 \\
\hline C14-C13-C12 & $120.26(19)$ & C14-C13-H13 & 119.9 \\
\hline C12-C13-H13 & 119.9 & C13-C14-C15 & $120.91(18)$ \\
\hline C13-C14-H14 & 119.5 & C15-C14-H14 & 119.5 \\
\hline C14-C15-C16 & $122.12(16)$ & C14-C15-C10 & $118.86(16)$ \\
\hline C16-C15-C10 & $119.02(15)$ & C7-C16-C15 & $121.79(16)$ \\
\hline
\end{tabular}


Table S14. Torsion angles $\left(^{\circ}\right)$ for $(S)-1$ a.

\begin{tabular}{llll} 
C5-O1-C1-C7 & $-66.62(18)$ & C5-O1-C1-C2 & $59.41(19)$ \\
C5-O1-C1-C3 & $177.89(14)$ & O1-C1-C3-C4 & $-64.92(17)$ \\
C7-C1-C3-C4 & $177.97(16)$ & C2-C1-C3-C4 & $52.9(2)$ \\
C1-O1-C5-O2 & $3.6(3)$ & C1-O1-C5-C6 & $-176.21(14)$ \\
O1-C1-C7-C16 & $-26.1(2)$ & C2-C1-C7-C16 & $-150.35(16)$ \\
C3-C1-C7-C16 & $85.91(18)$ & O1-C1-C7-C8 \\
C2-C1-C7-C8 & $34.7(2)$ & C3-C1-C7-C8 & $158.93(14)$ \\
C16-C7-C8-C9 & $-0.3(3)$ & C1-C7-C8-C9 & $-89.05(18)$ \\
C7-C8-C9-C10 & $0.2(3)$ & C8-C9-C10-C11 & $174.88(16)$ \\
C8-C9-C10-C15 & $0.5(3)$ & C9-C10-C11-C12 & $-179.53(18)$ \\
C15-C10-C11-C12 & $0.2(3)$ & C10-C11-C12-C13 & $-179.82(18)$ \\
C11-C12-C13-C14 & $-1.0(3)$ & C12-C13-C14-C15 & $0.9(3)$ \\
C13-C14-C15-C16 & $-179.14(16)$ & C13-C14-C15-C10 & $0.0(3)$ \\
C9-C10-C15-C14 & $178.85(16)$ & C11-C10-C15-C14 & $1.1(3)$ \\
C9-C10-C15-C16 & $-0.9(2)$ & C11-C10-C15-C16 & $-1.2(2)$ \\
C8-C7-C16-C15 & $-0.2(2)$ & C1-C7-C16-C15 & $179.06(16)$ \\
C14-C15-C16-C7 & $-178.95(15)$ & C10-C15-C16-C7 & $-175.20(14)$ \\
\hline
\end{tabular}

Table S15. Anisotropic atomic displacement parameters $\left(\AA^{2}\right)$ for $(S)-1$ a.

The anisotropic atomic displacement factor exponent takes the form: $-2 \pi^{2}\left[h^{2} a^{* 2} U_{11}+\ldots+2 h k a^{*} b^{*}\right.$ $\mathrm{U}_{12}$ ]

\begin{tabular}{lllllll} 
& $\mathbf{U}_{11}$ & $\mathbf{U}_{22}$ & $\mathbf{U}_{\mathbf{3 3}}$ & $\mathbf{U}_{\mathbf{2 3}}$ & $\mathbf{U}_{\mathbf{1 3}}$ & $\mathbf{U}_{\mathbf{1 2}}$ \\
$\mathrm{O} 1$ & $0.0306(6)$ & $0.0377(6)$ & $0.0366(6)$ & $0.0034(5)$ & $0.0016(4)$ & $-0.0007(5)$ \\
$\mathrm{O} 2$ & $0.0427(7)$ & $0.0672(10)$ & $0.0555(8)$ & $-0.0023(8)$ & $-0.0033(6)$ & $-0.0155(8)$ \\
$\mathrm{C} 1$ & $0.0318(7)$ & $0.0301(8)$ & $0.0353(8)$ & $-0.0008(7)$ & $0.0035(6)$ & $0.0007(6)$ \\
$\mathrm{C} 2$ & $0.0510(11)$ & $0.0386(10)$ & $0.0478(10)$ & $-0.0099(8)$ & $0.0134(8)$ & $-0.0023(8)$ \\
$\mathrm{C} 3$ & $0.0299(8)$ & $0.0387(9)$ & $0.0368(8)$ & $-0.0010(7)$ & $0.0029(6)$ & $-0.0005(7)$ \\
C4 & $0.0357(9)$ & $0.0512(11)$ & $0.0472(10)$ & $-0.0004(9)$ & $0.0085(7)$ & $-0.0061(8)$ \\
C5 & $0.0320(8)$ & $0.0560(12)$ & $0.0341(8)$ & $-0.0024(8)$ & $0.0037(6)$ & $-0.0019(8)$ \\
C6 & $0.0387(9)$ & $0.0745(15)$ & $0.0443(10)$ & $0.0088(10)$ & $0.0016(8)$ & $0.0103(10)$ \\
C7 & $0.0308(7)$ & $0.0296(8)$ & $0.0363(8)$ & $-0.0022(7)$ & $0.0025(6)$ & $-0.0018(6)$ \\
C8 & $0.0376(8)$ & $0.0283(8)$ & $0.0479(10)$ & $-0.0003(7)$ & $0.0071(7)$ & $0.0030(7)$ \\
C9 & $0.0420(9)$ & $0.0291(8)$ & $0.0478(10)$ & $0.0037(8)$ & $0.0036(7)$ & $0.0002(7)$ \\
C10 & $0.0334(8)$ & $0.0342(8)$ & $0.0391(8)$ & $0.0004(7)$ & $0.0025(6)$ & $-0.0048(7)$ \\
C11 & $0.0469(10)$ & $0.0434(10)$ & $0.0432(10)$ & $0.0055(8)$ & $0.0059(8)$ & $-0.0062(8)$ \\
C12 & $0.0482(10)$ & $0.0577(12)$ & $0.0407(9)$ & $-0.0002(9)$ & $0.0146(8)$ & $-0.0094(9)$ \\
C13 & $0.0395(9)$ & $0.0528(12)$ & $0.0448(10)$ & $-0.0086(8)$ & $0.0100(7)$ & $-0.0004(8)$ \\
C14 & $0.0337(8)$ & $0.0379(9)$ & $0.0425(9)$ & $-0.0033(8)$ & $0.0036(6)$ & $0.0010(7)$ \\
C15 & $0.0287(7)$ & $0.0307(8)$ & $0.0374(8)$ & $-0.0006(6)$ & $0.0013(6)$ & $-0.0029(6)$ \\
C16 & $0.0320(7)$ & $0.0282(8)$ & $0.0374(8)$ & $0.0006(6)$ & $0.0024(6)$ & $-0.0005(6)$ \\
\hline
\end{tabular}


Table S16. Hydrogen atomic coordinates and isotropic atomic displacement parameters $\left(\breve{\mathrm{A}}^{2}\right)$ for (S)-1a.

\begin{tabular}{lllll} 
& $\mathbf{x} / \mathbf{a}$ & $\mathbf{y} / \mathbf{b}$ & $\mathbf{z} / \mathbf{c}$ & $\mathbf{U}(\mathbf{e q})$ \\
H2A & 0.4184 & 0.1992 & 0.1532 & 0.068 \\
H2B & 0.4904 & 0.2184 & 0.2070 & 0.068 \\
H2C & 0.4633 & 0.3526 & 0.0791 & 0.068 \\
H3A & 0.4894 & 0.7708 & 0.3422 & 0.042 \\
H3B & 0.5249 & 0.5333 & 0.3617 & 0.042 \\
H4A & 0.5574 & 0.5617 & 0.1577 & 0.067 \\
H4B & 0.5763 & 0.7908 & 0.2354 & 0.067 \\
H4C & 0.5172 & 0.7877 & 0.1276 & 0.067 \\
H6A & 0.3521 & 0.8435 & -0.0533 & 0.079 \\
H6B & 0.3282 & 0.9605 & 0.0693 & 0.079 \\
H6C & 0.2836 & 0.7932 & -0.0204 & 0.079 \\
H8 & 0.4476 & 0.1286 & 0.3898 & 0.045 \\
H9 & 0.4046 & 0.0348 & 0.5722 & 0.048 \\
H11 & 0.3360 & 0.1347 & 0.7403 & 0.053 \\
H12 & 0.2695 & 0.3896 & 0.8202 & 0.058 \\
H13 & 0.2437 & 0.7343 & 0.7124 & 0.054 \\
H14 & 0.2866 & 0.8267 & 0.5286 & 0.046 \\
H16 & 0.3577 & 0.7322 & 0.3668 & 0.039 \\
\hline
\end{tabular}

\section{Crystal Structure Data for S-1d}

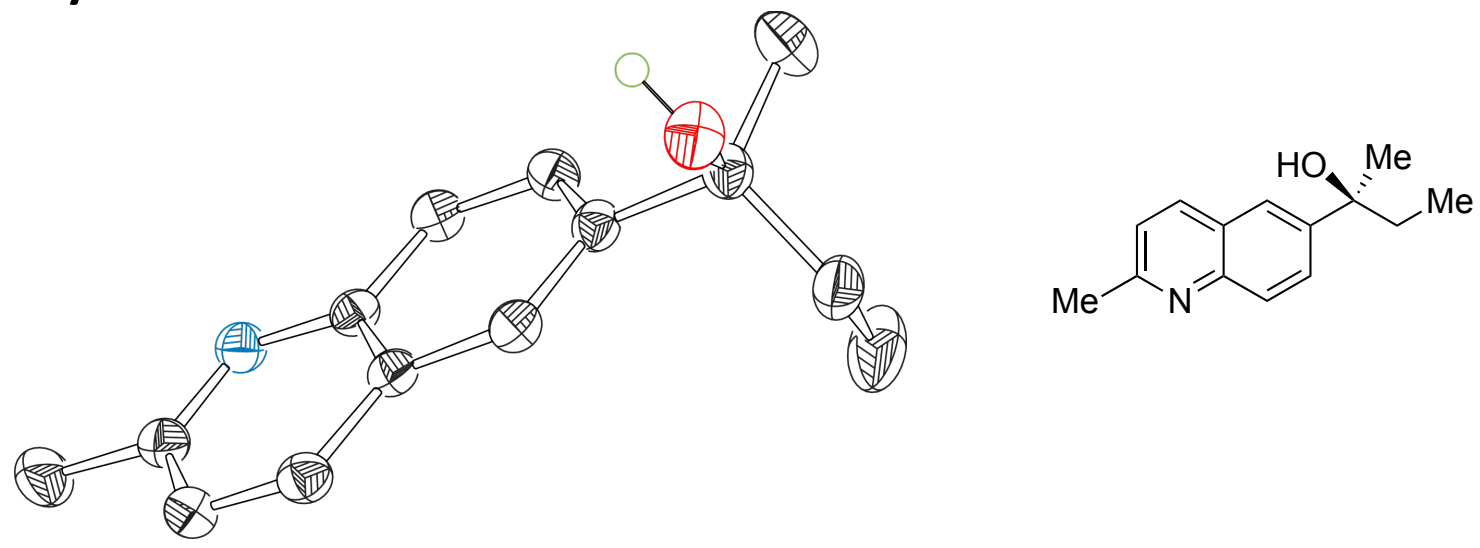

Table S17. Sample and crystal data for S-1d.

Identification code

Chemical formula

Formula weight

Temperature

Wavelength mary026

$\mathrm{C}_{14} \mathrm{H}_{17} \mathrm{NO}$

215.28

200(2) K

$1.54178 \AA$ 


\begin{tabular}{lll} 
Crystal size & $0.216 \times 0.425 \times 0.545 \mathrm{~mm}$ & \\
Crystal system & orthorhombic & \\
Space group & $\mathrm{P} 212121$ & \\
Unit cell dimensions & $\mathrm{a}=5.9000(2) \AA$ & $\alpha=90^{\circ}$ \\
& $\mathrm{b}=8.4404(3) \AA$ & $\beta=90^{\circ}$ \\
& $\mathrm{c}=23.7442(10) \AA$ & $\gamma=90^{\circ}$ \\
Volume & $1182.42(8) \AA^{3}$ & \\
$\mathbf{Z}$ & 4 & \\
Density (calculated) & $1.209 \mathrm{~g} / \mathrm{cm}^{3}$ & \\
Absorption coefficient & $0.591 \mathrm{~mm}^{-1}$ & \\
F(000) & 464 & \\
\hline
\end{tabular}

Table S18. Data collection and structure refinement for S-1d.

Theta range for data collection

Index ranges

Reflections collected

Independent reflections

Max. and min. transmission

Refinement method

Refinement program

Function minimized

Data / restraints / parameters

Goodness-of-fit on $\mathbf{F}^{2}$

$\Delta / \sigma_{\max }$

Final R indices

Weighting scheme

Absolute structure parameter

Extinction coefficient

Largest diff. peak and hole

R.M.S. deviation from mean
3.72 to $74.70^{\circ}$

$-7<=\mathrm{h}<=7,-10<=\mathrm{k}<=10,-29<=\mathrm{l}<=29$

19673

$2415[\mathrm{R}(\mathrm{int})=0.0388]$

0.8830 and 0.7390

Full-matrix least-squares on $\mathrm{F}^{2}$

SHELXL-2014/7 (Sheldrick, 2014)

$\sum \mathrm{w}\left(\mathrm{F}_{\mathrm{o}}^{2}-\mathrm{F}_{\mathrm{c}}^{2}\right)^{2}$

$2415 / 0 / 150$

1.020

0.001

2389 data; $\mathrm{I}>2 \sigma(\mathrm{I}) \quad \mathrm{R} 1=0.0333, \mathrm{wR} 2=0.0953$

all data

$\mathrm{R} 1=0.0337, \mathrm{wR} 2=0.0976$

$\mathrm{w}=1 /\left[\sigma^{2}\left(\mathrm{~F}_{\mathrm{o}}^{2}\right)+(0.0716 \mathrm{P})^{2}+0.1296 \mathrm{P}\right]$

where $\mathrm{P}=\left(\mathrm{F}_{\mathrm{o}}{ }^{2}+2 \mathrm{~F}_{\mathrm{c}}^{2}\right) / 3$

$-0.1(1)$

$0.0143(18)$

0.213 and $-0.198 \mathrm{e}^{-3}$

$0.044 \mathrm{e}^{-3}$

Table S19. Atomic coordinates and equivalent isotropic atomic displacement parameters $\left(\AA^{2}\right)$ for $\mathbf{S}$ 1d.

$\mathrm{U}(\mathrm{eq})$ is defined as one third of the trace of the orthogonalized $\mathrm{U}_{\mathrm{ij}}$ tensor.

\begin{tabular}{lllll}
\hline & $\mathbf{x} / \mathbf{a}$ & $\mathbf{y} / \mathbf{b}$ & $\mathbf{z} / \mathbf{c}$ & $\mathbf{U}(\mathbf{e q})$ \\
$\mathrm{N} 1$ & $0.7994(2)$ & $0.02782(15)$ & $0.06577(6)$ & $0.0283(3)$ \\
$\mathrm{O} 1$ & $0.37311(19)$ & $0.72139(14)$ & $0.84869(5)$ & $0.0341(3)$ \\
$\mathrm{C} 1$ & $0.7123(4)$ & $0.1740(2)$ & $0.15036(7)$ & $0.0426(4)$ \\
$\mathrm{C} 2$ & $0.6518(3)$ & $0.12030(18)$ & $0.09177(6)$ & $0.0297(3)$ \\
$\mathrm{C} 3$ & $0.4435(3)$ & $0.16671(18)$ & $0.06700(7)$ & $0.0309(4)$
\end{tabular}




\begin{tabular}{lllll} 
C4 & $0.3886(3)$ & $0.11487(18)$ & $0.01428(6)$ & $0.0275(3)$ \\
C5 & $0.5437(3)$ & $0.01707(16)$ & $0.98464(6)$ & $0.0244(3)$ \\
C6 & $0.5001(2)$ & $0.95546(17)$ & $0.93022(6)$ & $0.0256(3)$ \\
C7 & $0.6555(3)$ & $0.86111(17)$ & $0.90266(6)$ & $0.0254(3)$ \\
C8 & $0.8641(3)$ & $0.82844(17)$ & $0.92987(6)$ & $0.0275(3)$ \\
C9 & $0.9091(2)$ & $0.88311(18)$ & $0.98318(6)$ & $0.0275(3)$ \\
C10 & $0.7501(3)$ & $0.97764(16)$ & $0.01203(6)$ & $0.0243(3)$ \\
C11 & $0.5951(3)$ & $0.79050(17)$ & $0.84508(6)$ & $0.0273(3)$ \\
C12 & $0.7606(3)$ & $0.6636(2)$ & $0.82559(7)$ & $0.0408(4)$ \\
C13 & $0.5692(3)$ & $0.9218(2)$ & $0.80068(7)$ & $0.0376(4)$ \\
C14 & $0.7760(5)$ & $0.0252(3)$ & $0.79252(8)$ & $0.0610(7)$ \\
\hline
\end{tabular}

Table S20. Bond lengths (Å) for S-1d.

\begin{tabular}{llll}
$\mathrm{N} 1-\mathrm{C} 2$ & $1.322(2)$ & $\mathrm{N} 1-\mathrm{C} 10$ & $1.376(2)$ \\
$\mathrm{O} 1-\mathrm{C} 11$ & $1.4365(19)$ & $\mathrm{O} 1-\mathrm{H} 1$ & 0.84 \\
$\mathrm{C} 1-\mathrm{C} 2$ & $1.506(2)$ & $\mathrm{C} 1-\mathrm{H} 1 \mathrm{~A}$ & 0.98 \\
$\mathrm{C} 1-\mathrm{H} 1 \mathrm{~B}$ & 0.98 & $\mathrm{C} 1-\mathrm{H} 1 \mathrm{C}$ & 0.98 \\
$\mathrm{C} 2-\mathrm{C} 3$ & $1.418(2)$ & $\mathrm{C} 3-\mathrm{C} 4$ & $1.365(2)$ \\
$\mathrm{C} 3-\mathrm{H} 3$ & $\mathrm{C} 4-\mathrm{C} 5$ & $1.419(2)$ \\
$\mathrm{C} 4-\mathrm{H} 4$ & 0.95 & $\mathrm{C} 5-\mathrm{C} 6$ & $1.416(2)$ \\
$\mathrm{C} 5-\mathrm{C} 10$ & 0.95 & $\mathrm{C} 6-\mathrm{C} 7$ & $1.379(2)$ \\
$\mathrm{C} 6-\mathrm{H} 6$ & $1.420(2)$ & $\mathrm{C} 7-\mathrm{C} 8$ & $1.417(2)$ \\
$\mathrm{C} 7-\mathrm{C} 11$ & $\mathrm{C} 8-\mathrm{C} 9$ & $1.373(2)$ \\
$\mathrm{C} 8-\mathrm{H} 8$ & 0.95 & $\mathrm{C} 9-\mathrm{C} 10$ & $1.409(2)$ \\
$\mathrm{C} 9-\mathrm{H} 9$ & $1.5335(19)$ & $\mathrm{C} 11-\mathrm{C} 12$ & $1.522(2)$ \\
$\mathrm{C} 11-\mathrm{C} 13$ & 0.95 & $\mathrm{C} 12-\mathrm{H} 12 \mathrm{~A}$ & 0.98 \\
$\mathrm{C} 12-\mathrm{H} 12 \mathrm{~B}$ & 0.95 & $\mathrm{C} 12-\mathrm{H} 12 \mathrm{C}$ & 0.98 \\
$\mathrm{C} 13-\mathrm{C} 14$ & $1.537(2)$ & $\mathrm{C} 13-\mathrm{H} 13 \mathrm{~A}$ & 0.99 \\
$\mathrm{C} 13-\mathrm{H} 13 \mathrm{~B}$ & 0.98 & $\mathrm{C} 14-\mathrm{H} 14 \mathrm{~A}$ & 0.98 \\
$\mathrm{C} 14-\mathrm{H} 14 \mathrm{~B}$ & $1.512(3)$ & $\mathrm{C} 14-\mathrm{H} 14 \mathrm{C}$ & 0.98 \\
\hline
\end{tabular}

Table S21. Bond angles $\left(^{\circ}\right)$ for S-1d.

$\begin{array}{llll}\text { C2-N1-C10 } & 118.39(14) & \text { C11-O1-H1 } & 109.5 \\ \text { C2-C1-H1A } & 109.5 & \text { C2-C1-H1B } & 109.5 \\ \text { H1A-C1-H1B } & 109.5 & \text { C2-C1-H1C } & 109.5 \\ \text { H1A-C1-H1C } & 109.5 & \text { H1B-C1-H1C } & 109.5 \\ \text { N1-C2-C3 } & 122.71(14) & \text { N1-C2-C1 } & 116.91(15) \\ \text { C3-C2-C1 } & 120.37(15) & \text { C4-C3-C2 } & 119.84(14) \\ \text { C4-C3-H3 } & 120.1 & \text { C2-C3-H3 } & 120.1 \\ \text { C3-C4-C5 } & 119.22(14) & \text { C3-C4-H4 } & 120.4 \\ \text { C5-C4-H4 } & 120.4 & \text { C6-C5-C4 } & 123.29(14)\end{array}$




\begin{tabular}{llll} 
C6-C5-C10 & $119.17(13)$ & C4-C5-C10 & $117.53(13)$ \\
C7-C6-C5 & $121.61(13)$ & C7-C6-H6 & 119.2 \\
C5-C6-H6 & 119.2 & C6-C7-C8 & $118.24(13)$ \\
C6-C7-C11 & $119.53(13)$ & C8-C7-C11 & $122.19(13)$ \\
C9-C8-C7 & $121.53(14)$ & C9-C8-H8 & 119.2 \\
C7-C8-H8 & 119.2 & C8-C9-C10 & $120.62(14)$ \\
C8-C9-H9 & 119.7 & C10-C9-H9 & 119.7 \\
N1-C10-C9 & $118.97(14)$ & N1-C10-C5 & $122.28(14)$ \\
C9-C10-C5 & $118.75(13)$ & O1-C11-C12 & $108.50(13)$ \\
O1-C11-C7 & $108.45(11)$ & C12-C11-C7 & $113.30(13)$ \\
O1-C11-C13 & $104.08(12)$ & C12-C11-C13 & $111.28(14)$ \\
C7-C11-C13 & $110.75(12)$ & C11-C12-H12A & 109.5 \\
C11-C12-H12B & 109.5 & H12A-C12-H12B & 109.5 \\
C11-C12-H12C & 109.5 & H12A-C12-H12C & 109.5 \\
H12B-C12-H12C & 109.5 & C14-C13-C11 & $115.07(16)$ \\
C14-C13-H13A & 108.5 & C11-C13-H13A & 108.5 \\
C14-C13-H13B & 108.5 & C11-C13-H13B & 108.5 \\
H13A-C13-H13B & 107.5 & C13-C14-H14A & 109.5 \\
C13-C14-H14B & 109.5 & H14A-C14-H14B & 109.5 \\
C13-C14-H14C & 109.5 & H14A-C14-H14C & 109.5 \\
H14B-C14-H14C & 109.5 & & \\
\hline
\end{tabular}

Table S22. Torsion angles $\left(^{\circ}\right)$ for $\mathbf{S - 1 d}$.

\begin{tabular}{llll} 
C10-N1-C2-C3 & $1.4(2)$ & C10-N1-C2-C1 & $-179.53(14)$ \\
N1-C2-C3-C4 & $0.3(2)$ & C1-C2-C3-C4 & $-178.76(15)$ \\
C2-C3-C4-C5 & $-1.1(2)$ & C3-C4-C5-C6 & $178.91(13)$ \\
C3-C4-C5-C10 & $0.2(2)$ & C4-C5-C6-C7 & $179.80(14)$ \\
C10-C5-C6-C7 & $-1.5(2)$ & C5-C6-C7-C8 & $-0.9(2)$ \\
C5-C6-C7-C11 & $176.90(12)$ & C6-C7-C8-C9 & $2.6(2)$ \\
C11-C7-C8-C9 & $-175.20(13)$ & C7-C8-C9-C10 & $-1.7(2)$ \\
C2-N1-C10-C9 & $178.04(13)$ & C2-N1-C10-C5 & $-2.3(2)$ \\
C8-C9-C10-N1 & $178.83(13)$ & C8-C9-C10-C5 & $-0.8(2)$ \\
C6-C5-C10-N1 & $-177.24(13)$ & C4-C5-C10-N1 & $1.5(2)$ \\
C6-C5-C10-C9 & $2.4(2)$ & C4-C5-C10-C9 & $-178.84(13)$ \\
C6-C7-C11-O1 & $-47.66(18)$ & C8-C7-C11-O1 & $130.09(14)$ \\
C6-C7-C11-C12 & $-168.19(14)$ & C8-C7-C11-C12 & $9.6(2)$ \\
C6-C7-C11-C13 & $65.96(18)$ & C8-C7-C11-C13 & $-116.29(16)$ \\
O1-C11-C13-C14 & $174.47(15)$ & C12-C11-C13-C14 & $-68.9(2)$ \\
C7-C11-C13-C14 & $58.1(2)$ & & \\
\hline
\end{tabular}

Table S23. Anisotropic atomic displacement parameters $\left(\AA^{2}\right)$ for S-1d.

The anisotropic atomic displacement factor exponent takes the form: $-2 \pi^{2}\left[h^{2} a^{* 2} U_{11}+\ldots+2 h k a^{*} b^{*}\right.$ $\left.\mathrm{U}_{12}\right]$ 


\begin{tabular}{lllllll} 
& $\mathbf{U}_{11}$ & $\mathbf{U}_{22}$ & $\mathbf{U}_{33}$ & $\mathbf{U}_{23}$ & $\mathbf{U}_{13}$ & $\mathbf{U}_{12}$ \\
$\mathrm{~N} 1$ & $0.0306(7)$ & $0.0276(6)$ & $0.0268(6)$ & $-0.0002(5)$ & $-0.0030(5)$ & $0.0005(5)$ \\
$\mathrm{O} 1$ & $0.0311(6)$ & $0.0391(6)$ & $0.0321(6)$ & $-0.0004(4)$ & $-0.0023(4)$ & $-0.0084(5)$ \\
$\mathrm{C} 1$ & $0.0553(11)$ & $0.0413(9)$ & $0.0311(8)$ & $-0.0072(7)$ & $-0.0055(8)$ & $0.0079(8)$ \\
$\mathrm{C} 2$ & $0.0365(8)$ & $0.0247(6)$ & $0.0279(7)$ & $0.0000(6)$ & $-0.0006(6)$ & $-0.0004(6)$ \\
$\mathrm{C} 3$ & $0.0348(8)$ & $0.0276(7)$ & $0.0301(7)$ & $-0.0007(6)$ & $0.0041(6)$ & $0.0036(6)$ \\
C4 & $0.0253(7)$ & $0.0265(6)$ & $0.0308(7)$ & $0.0018(6)$ & $-0.0003(6)$ & $0.0023(6)$ \\
C5 & $0.0242(7)$ & $0.0225(6)$ & $0.0264(7)$ & $0.0033(5)$ & $0.0005(5)$ & $-0.0006(6)$ \\
C6 & $0.0230(7)$ & $0.0270(7)$ & $0.0268(7)$ & $0.0021(5)$ & $-0.0021(6)$ & $-0.0004(6)$ \\
C7 & $0.0262(7)$ & $0.0257(6)$ & $0.0242(6)$ & $0.0019(5)$ & $0.0011(5)$ & $-0.0031(6)$ \\
C8 & $0.0235(7)$ & $0.0290(7)$ & $0.0301(7)$ & $-0.0010(6)$ & $0.0016(6)$ & $0.0023(6)$ \\
C9 & $0.0226(7)$ & $0.0293(7)$ & $0.0305(7)$ & $0.0011(6)$ & $-0.0028(5)$ & $0.0006(6)$ \\
C10 & $0.0245(7)$ & $0.0222(6)$ & $0.0260(7)$ & $0.0021(5)$ & $-0.0016(5)$ & $-0.0022(6)$ \\
C11 & $0.0278(7)$ & $0.0293(7)$ & $0.0249(7)$ & $-0.0013(5)$ & $-0.0009(5)$ & $-0.0025(6)$ \\
C12 & $0.0406(9)$ & $0.0441(9)$ & $0.0377(8)$ & $-0.0141(7)$ & $-0.0021(7)$ & $0.0055(8)$ \\
C13 & $0.0509(10)$ & $0.0376(8)$ & $0.0242(7)$ & $0.0019(6)$ & $-0.0016(7)$ & $-0.0046(8)$ \\
C14 & $0.0884(17)$ & $0.0595(12)$ & $0.0351(9)$ & $0.0044(8)$ & $0.0028(11)$ & $-0.0373(13)$ \\
\hline
\end{tabular}

Table S24. Hydrogen atomic coordinates and isotropic atomic displacement parameters $\left(\AA^{2}\right)$ for $\mathbf{S}-\mathbf{1 d}$.

\begin{tabular}{lllll} 
& $\mathbf{x} / \mathbf{a}$ & $\mathbf{y} / \mathbf{b}$ & $\mathbf{z} / \mathbf{c}$ & $\mathbf{U}(\mathbf{e q})$ \\
H1 & 0.3743 & 0.6477 & 0.8725 & 0.051 \\
H1A & 0.6046 & 1.1287 & 1.1773 & 0.064 \\
H1B & 0.7057 & 1.2898 & 1.1523 & 0.064 \\
H1C & 0.8659 & 1.1382 & 1.1596 & 0.064 \\
H3 & 0.3423 & 1.2337 & 1.0870 & 0.037 \\
H4 & 0.2483 & 1.1439 & 0.9976 & 0.033 \\
H6 & 0.3605 & 0.9797 & 0.9123 & 0.031 \\
H8 & 0.9754 & 0.7673 & 0.9108 & 0.033 \\
H9 & 1.0488 & 0.8570 & 1.0008 & 0.033 \\
H12A & 0.7689 & 0.5794 & 0.8539 & 0.061 \\
H12B & 0.9111 & 0.7106 & 0.8205 & 0.061 \\
H12C & 0.7085 & 0.6189 & 0.7898 & 0.061 \\
H13A & 0.5308 & 0.8720 & 0.7642 & 0.045 \\
H13B & 0.4402 & 0.9903 & 0.8116 & 0.045 \\
H14A & 0.8085 & 1.0824 & 0.8275 & 0.091 \\
H14B & 0.7473 & 1.1013 & 0.7622 & 0.091 \\
H14C & 0.9062 & 0.9587 & 0.7825 & 0.091 \\
\hline
\end{tabular}




\section{References}

${ }^{1}$ Pangborn, A. B.; Giardello, M. A.; Grubbs, R. H.; Rosen, R. K.; Timmers, F. J. Organometallics 1996, 15, 1518.

2 Walsh, P.J. U.S. Patent 0191345 A1, October 9, 2003.

${ }^{3}$ Langer, F.; Schwink, L.; Devasagayaraj, A.; Chavant, P.-Y.; Knochel P. J. Org. Chem., 1996, 61, 8229.

${ }^{4}$ Waltz, K.M.; Gavenonis, J.; Walsh, P.J.; Angew. Chem. Int. Ed. 2002, 41, 3697.

${ }^{5}$ Williams, F.J.; Crote, R.E.; Jarvo, E.R.; Chem. Commun. 2012, 48, 1496.

${ }^{6}$ Enquist, P.-A.; Lindh, J.; Nilsson, P.; Larhed, M. Green Chem. 2006, 8, 338.

${ }^{7}$ (a) Jeon, S.-J.; Li, H.M.; Walsh, P.J. J. Am. Chem. Soc. 2005, 127, 16416. (b) Derwing, C.; Hoppe, D. Synthesis 1996, 149.

${ }^{8}$ Li, H.M.; Walsh, P.J. J. Am. Chem. Soc. 2004, 126, 6538.

${ }^{9}$ García, C.; Walsh, P.J. Org. Lett. 2003, 5, 3641. 
9เ9LO

$8 E L L^{\circ} 0$

098 ${ }^{\circ} O^{\circ}$

LL69' -

6LOZ乙

LOZZZ

เOEZ Z

ย乙ยて て

レて๖でて

6ยG乙 乙-

9992 乙

GSLZZ

8LLZ Z

9L8Z乙

LO०E 2

LZEL'9

9GEL'9

G8EL'9

866L'9-

ZZ08'9]

เ७08.9]

나8 9

LEL8.9-

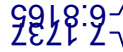

$\rightarrow 9 \angle L L$

$96 L t L$

$\angle L 8 L^{\circ} L$

$606 \mathrm{~L} L$

It6L L

हІОهО 009Z L

99Et $L$

98Et $L$

S6t大 $L$

ำ

L6St' $L$

$90 \angle D^{\circ} L$

$8 Z \angle T^{\circ} L$

OL89 L

†G69. $L$

ZELL'L

9GLLL

ZL8L $L$

LO6LL

G0Z8 $L$

8ZZ8 $L$

¿E\&8 $\angle-$

ZGE8 L

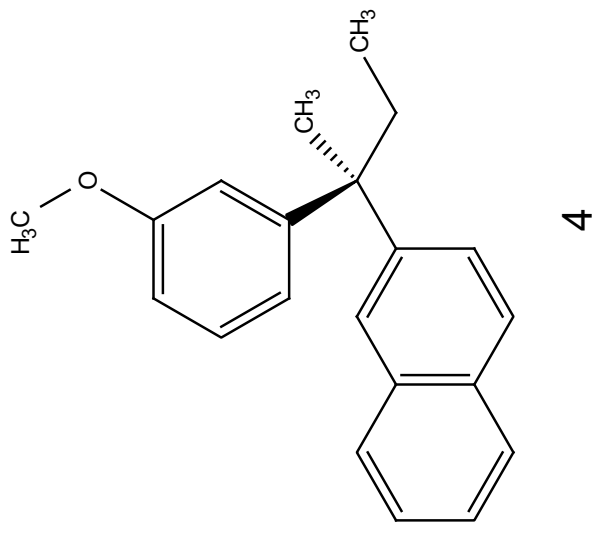


8998. 6 -

GL88.9Z-

$\angle \dashv \subseteq 8^{\circ} \varepsilon \varepsilon-$

ง8†8'9t-

8LGZ ९९-

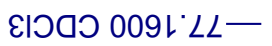

\&89Zㅇㄴ-

아란G6 $\angle 8^{\circ} \nabla Z \mathrm{~L}$

$\varepsilon \varepsilon \nabla G$ 'GZL]

96Z6'GZᄂ 는

$90 Z Z$ LZL

8tLGLZL-F

$\angle 819^{\circ} \angle Z L$

$\angle G Z L 8 Z 1]$

†७6 $8 Z$ -

LE88'LEL

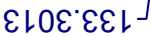

8เட89ヤレー

98LtレGL

L†ย๋ $6 \varsigma$ -
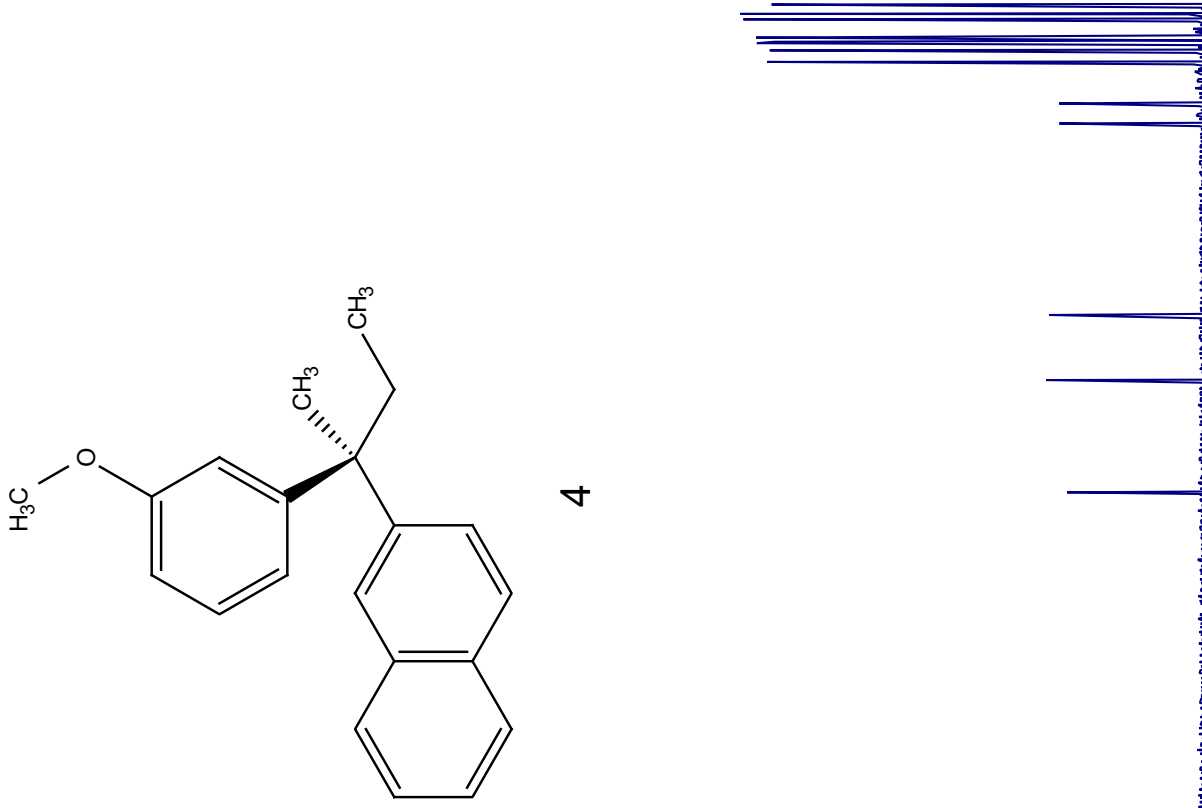
606L'L

L66L L

OtOZ L

E60Z L-

$99 \mathrm{~L} Z \mathrm{~L}$

OLZZ L

ISZZ L-

$06 \varepsilon Z^{\circ} L$

LEDZ $L-$

$\varepsilon \angle \nabla Z \angle-$

6EG乙 $L$

Z09Z L-

$9+9 Z^{\circ} L$

$6 \angle L Z^{\circ} \angle-$

$6 \varepsilon 8 Z^{\circ} L$

$\angle Z 6 Z$ L

Z962 L-

$066 Z^{\circ} L$

ZOLE L

E†レE L

ZLLE $L$

$\nabla \nabla \nabla \nabla^{\circ} L$

Z৪t७ L

6 เ $9 t^{\circ} L$

$\nabla G 9 t^{\circ} L$

$089 t^{\circ} L$

E08t L

E987 $L$

$\angle L 6 T^{\circ} L$

SOOS $L$

乙†0Gं

GLIGn

乙LZS익

GZOL先-

乙๖乙L

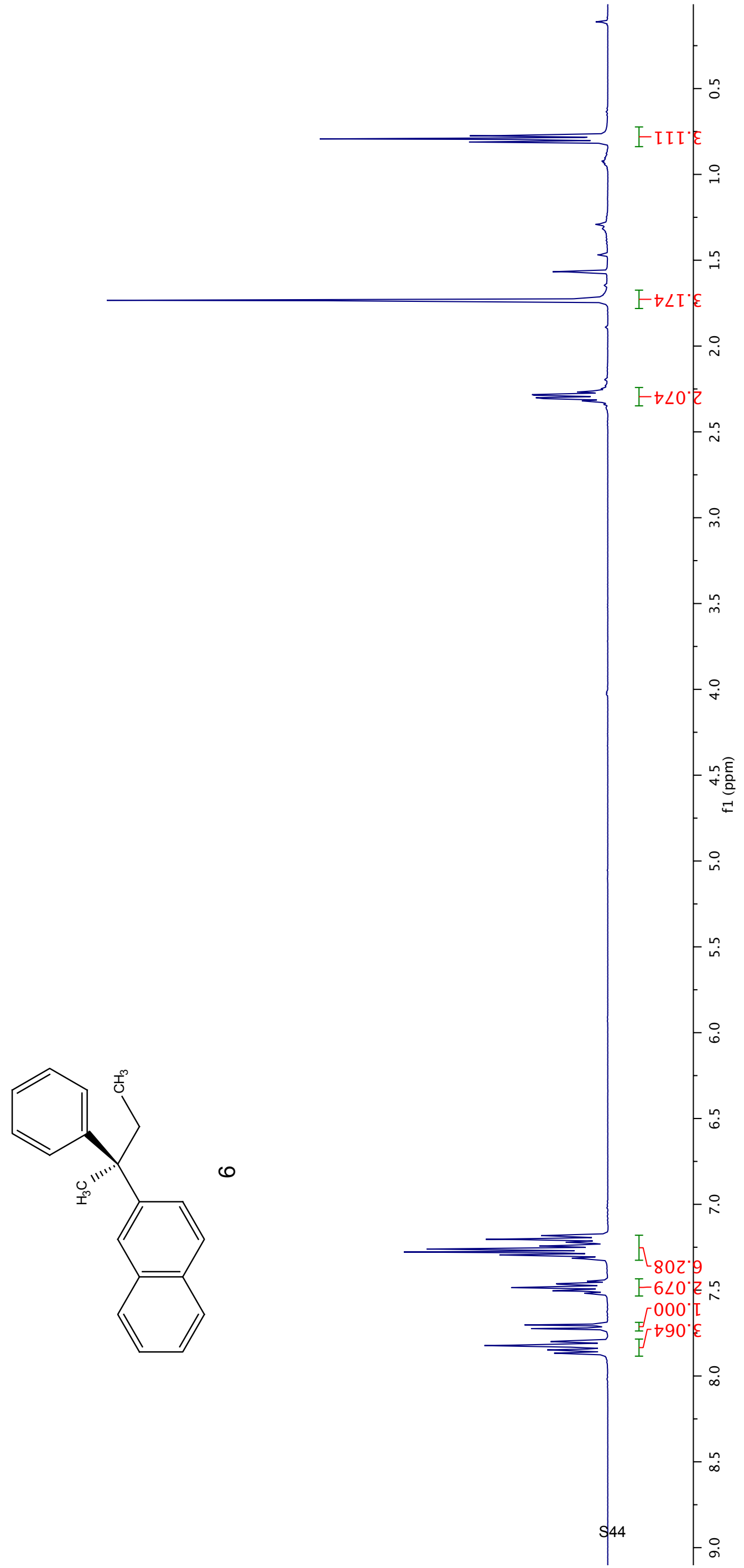

996L 중

6L08

क्षा8

E्टZयL

郚考路

sis

6458

to@ 

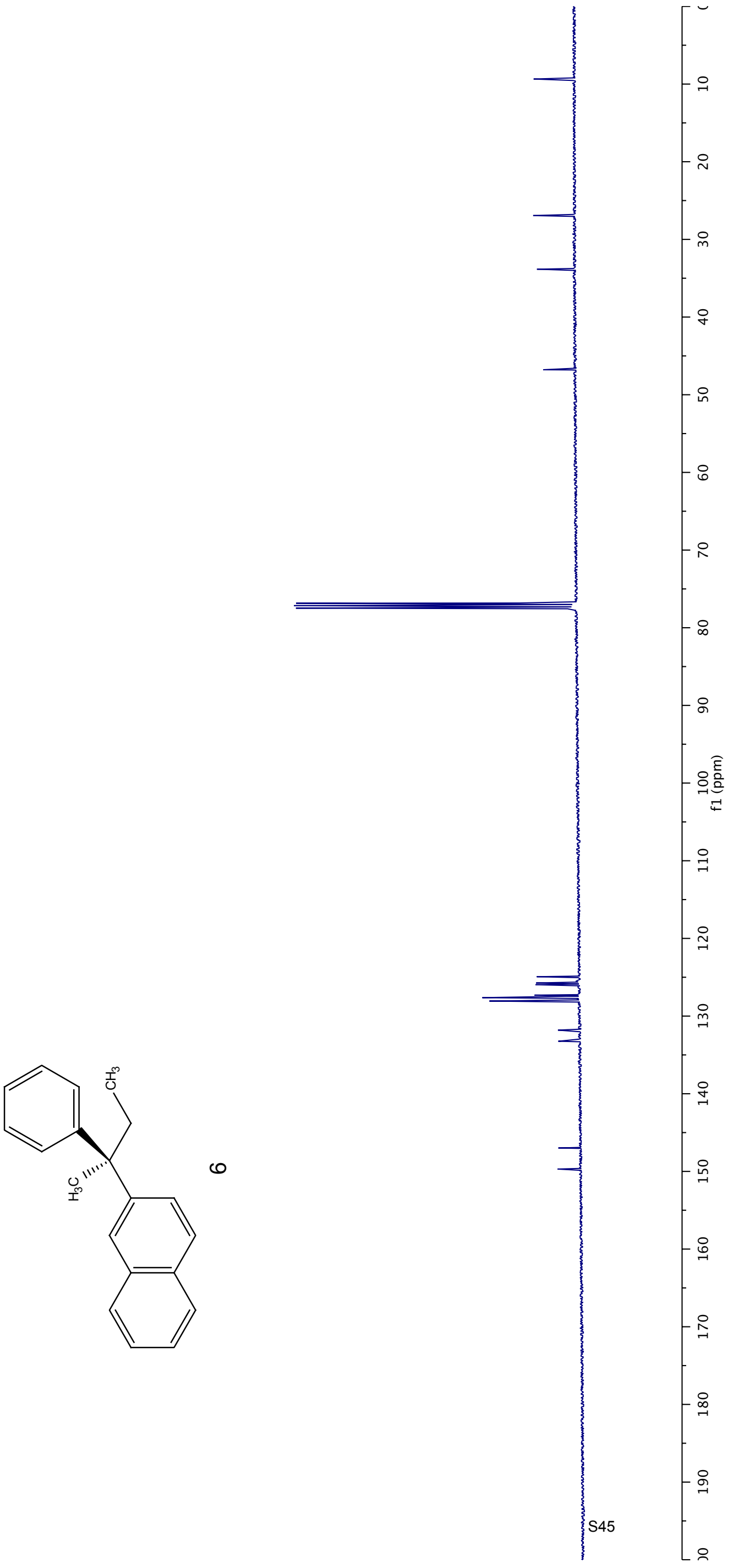
†6เL ${ }^{\circ} 0$

$919 L^{\circ} 0$

$8 \varepsilon L 0^{\circ}$

90<l'Z

8Z81'

626L'乙

IS6L'Z-

6†0Z乙

$\angle 91 Z$ Z

与8乙乙 乙

ง৪ะ乙 乙-

80tZ $Z$

LOSZ Z -

6Z9Z 乙

0ะเ6

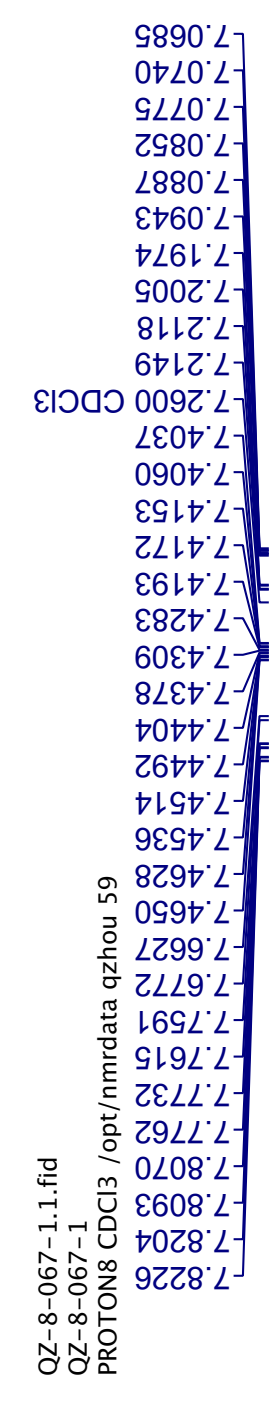

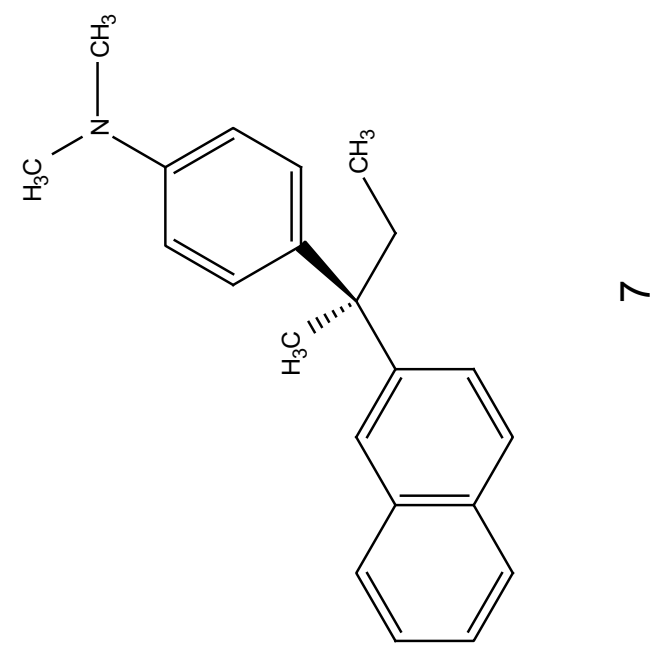

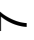


$96+0: 2 Z-$

$0 \angle L 0^{\circ} \downarrow \varepsilon-$

$0708.0 t-$

8688 St-

88tยてルーー

$0 Z Z 8^{\circ} \mathrm{ZL}$

88LE GZL

00Z8 GZL -

$\angle L \nabla T^{\circ} \angle Z L$

6เ9t LZL]

096t LZL I

IZOL'8ZL-

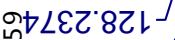

تZ96L LEL

올. $\varepsilon \varepsilon$

NELLL'LEL-

\section{.}

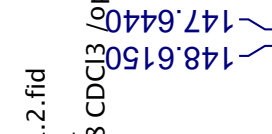

거

ํํㅇㅇㅛ

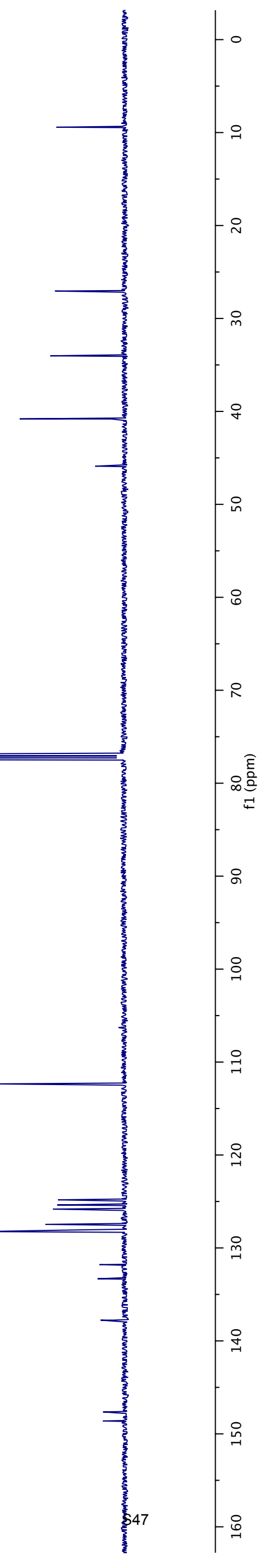



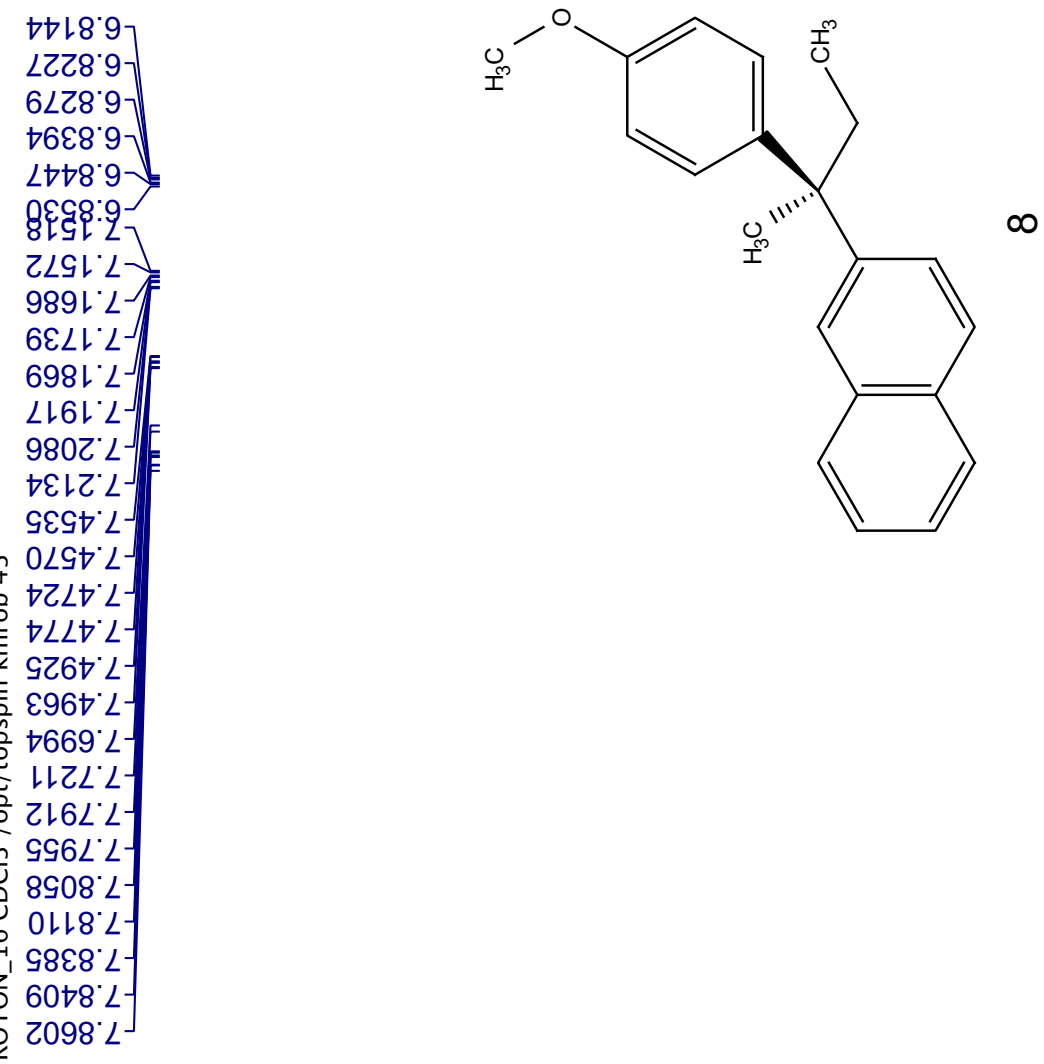

$\infty$

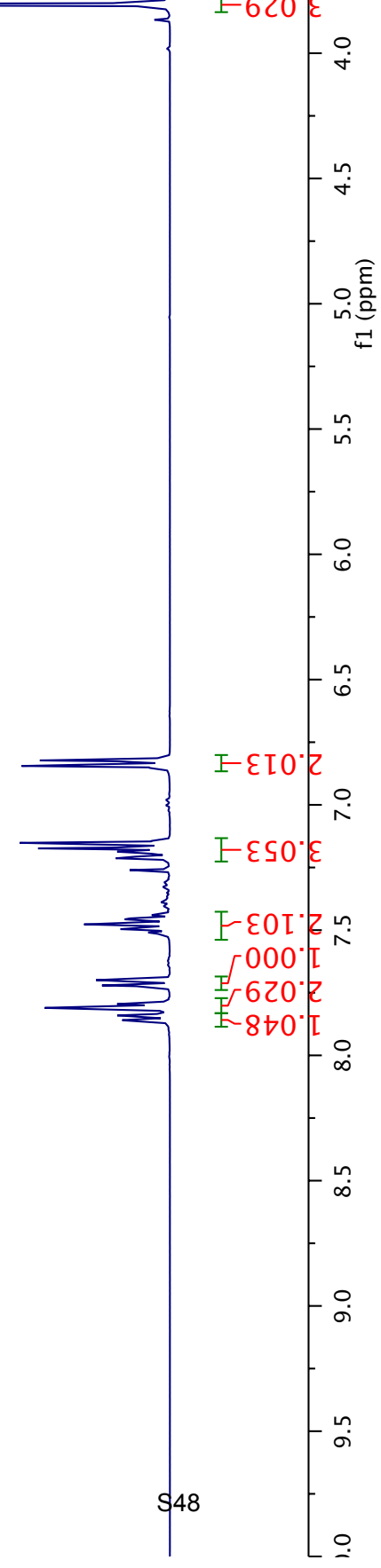


089ع 6 -

$0690^{\circ} \angle Z-$

เ6L6' $8 \varepsilon-$

$1601 \cdot 97-$

6เ8Z:99-
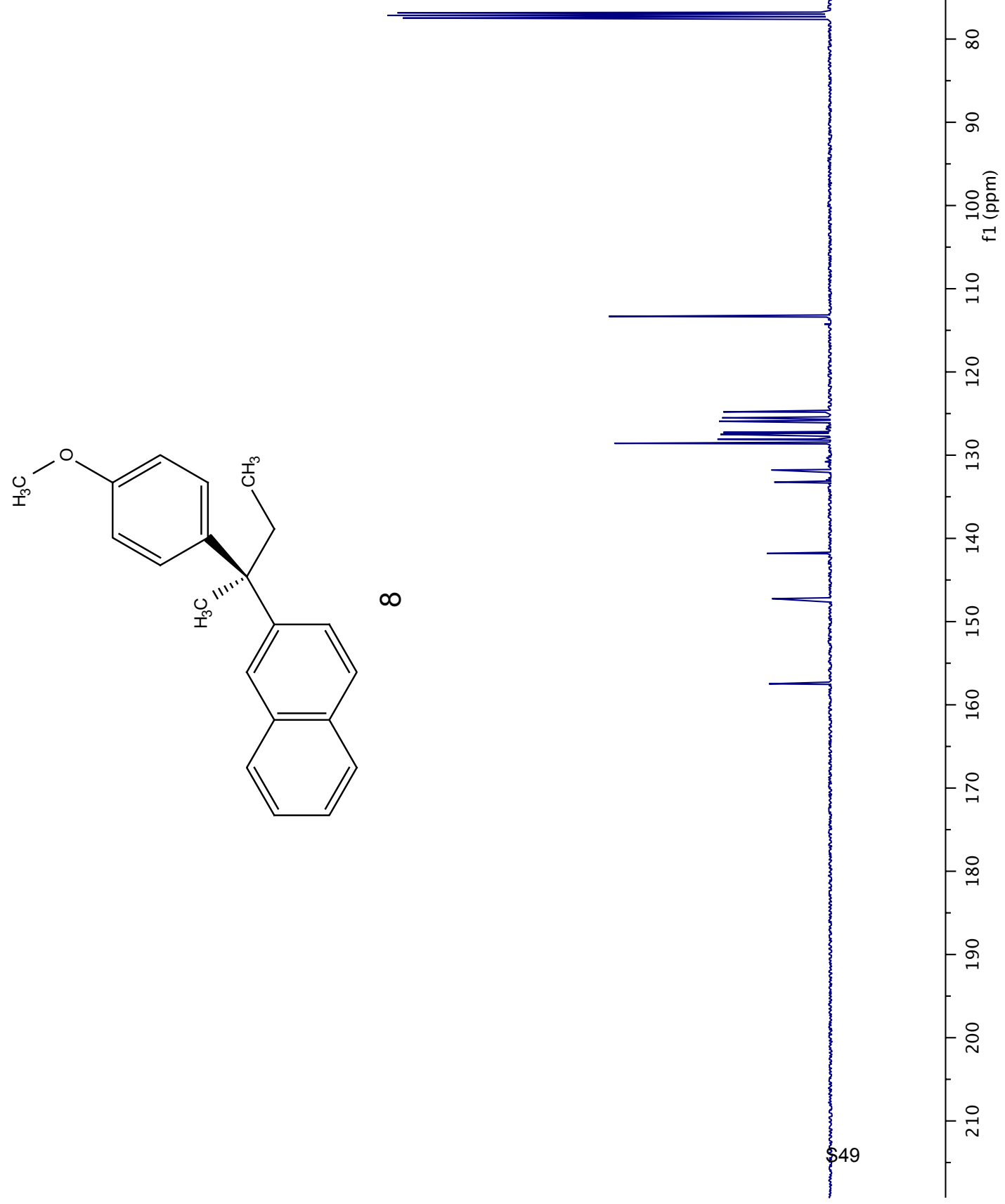
G88L 0

$\angle 008^{\circ} 0$

ocl8: 0

L8LLI-

L6LZZ

6เEZ

LレZZ

レナつてて

$\nabla \triangleright G Z$ ¿

0Z9Z

†99乙

$9 \triangleright L Z 乙$

68L乙 Z

$\angle 98 Z^{\circ} 乙-$

896乙 乙-

Z66乙 乙-

乙60ย乙-

†乙८६ ¿

เレIL

Et9l:L

ESLLL

L6LLL

8Z8L L

$006 L^{\circ} \mathrm{L}$

GE6L'L-

$786 \mathrm{~L}^{\circ} \mathrm{L}$

ยІวดว 009Z L

$\left.G \rightarrow \angle Z^{\circ} \angle\right]$

$818 C^{\circ} L$

†レL $L$

$\left.8 \varepsilon \angle \nabla^{\circ} L\right]$

乙E8๐ $L]$

998t $L$

096t $L$

$\angle 86 t^{\circ} \angle$

9ZOG $\angle$

$\triangle S 0 S^{\circ} L$

ESIGL

I8LG'L-

ZLZG'L-

96ZG $L$

$\angle G Z L L$

LOTL'L-

ZE08 $\angle$

$0908^{\circ} \mathrm{L}$

万

ก $9628 \mathrm{~L}$

की 6998 L-

N $1698^{\circ} \mathrm{L}$

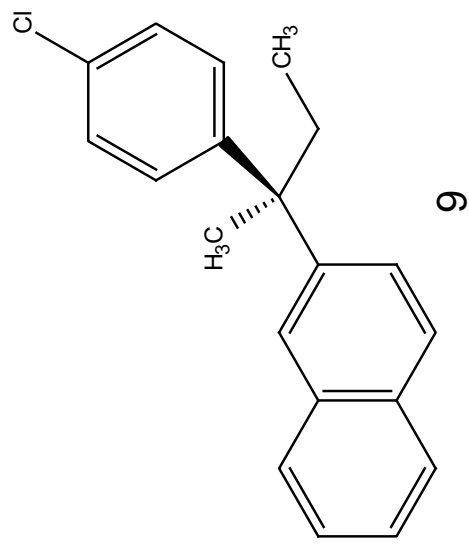

a

\section{$\widehat{\bar{\varepsilon}}$}

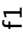

n?

$\stackrel{0}{\circ}$

in 
ع88ว 6-

عE\&6.92-

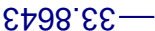

†999'9॰-

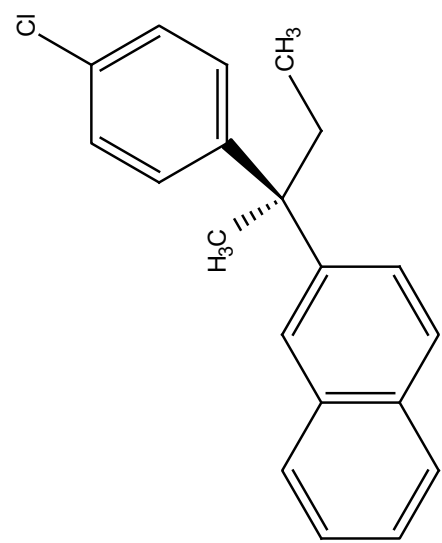

$\infty$

8986"大乙L

68ZL'GZL

8Z0L'9ZL]

$\left.\angle L 00^{\circ} \angle Z L\right]$

SStG' $\angle Z L]$

$\angle 86 L^{\circ} \angle Z L$ -

$860 L^{\circ} 8 Z 1-$

$8 \varepsilon 8 L^{\circ} 8 Z \mathrm{I}$

ᄂZ60'6Zᄂ

E9SG' $L E L$

90L6'LEL

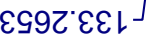

م́

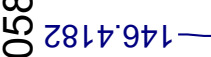

め'88928tレ-

N

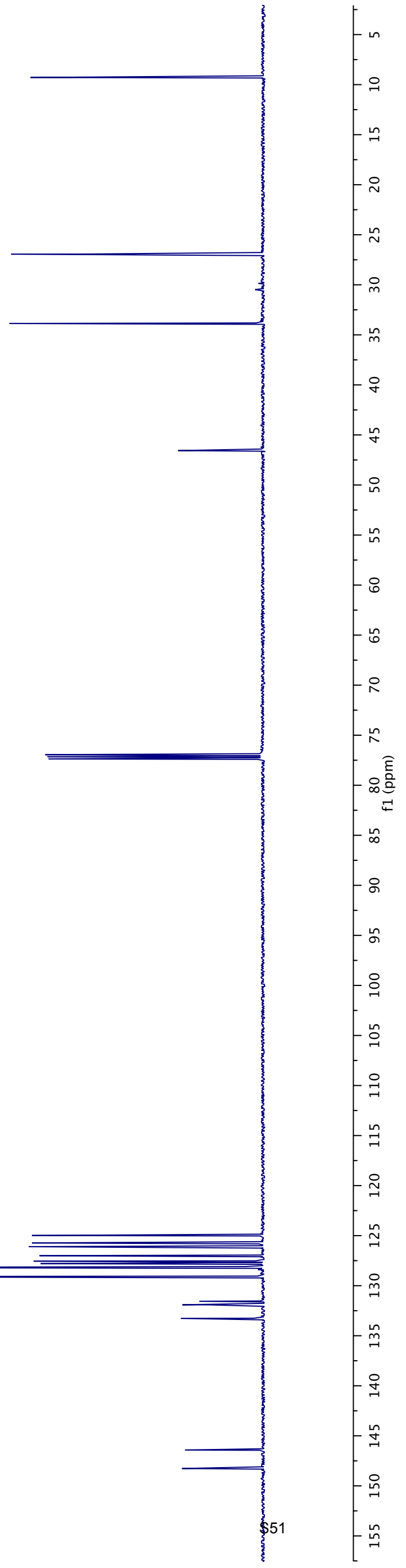




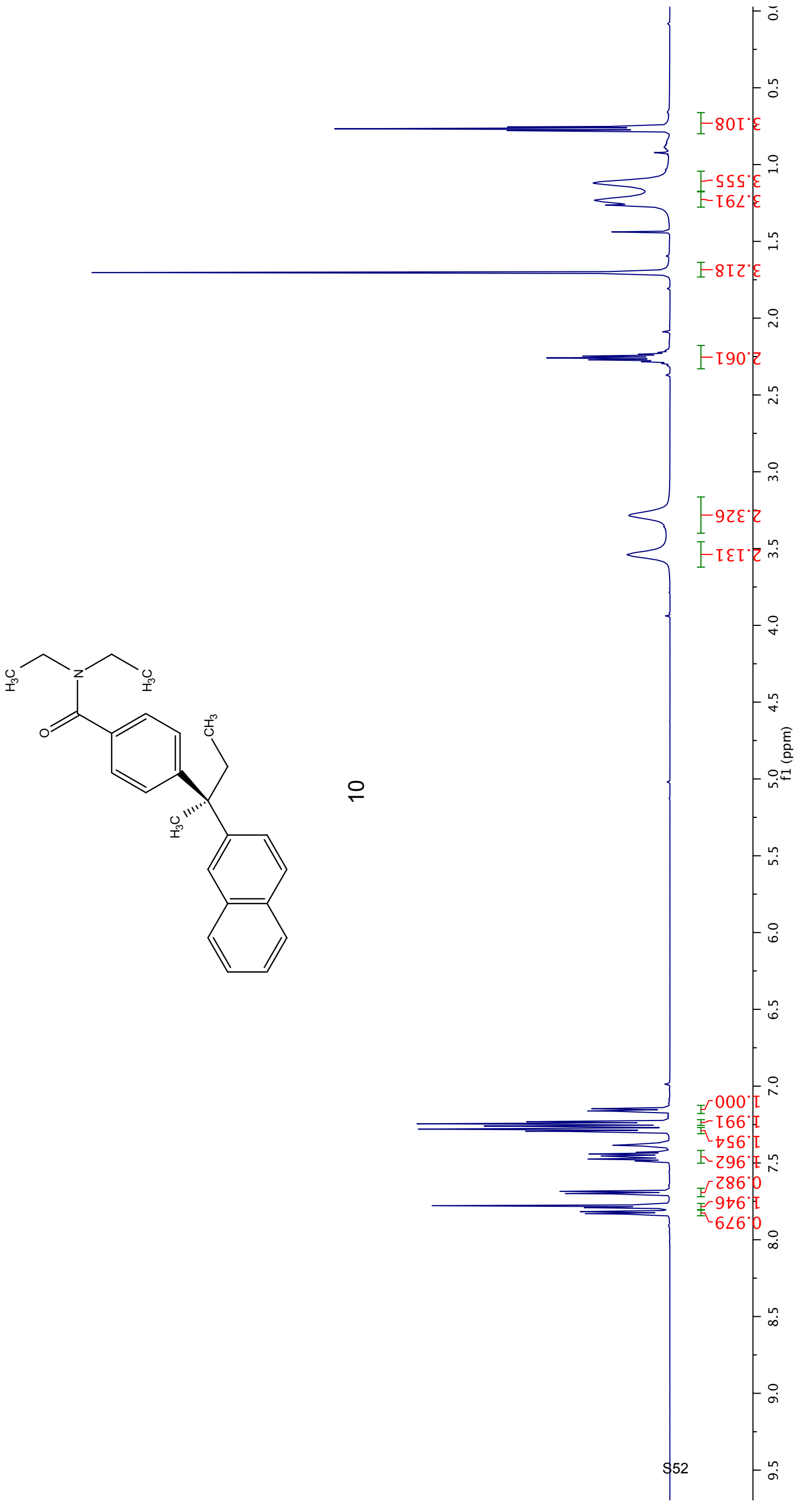

6Z0Lレー

カ乙レ乙

ง๖ZZて

५९E乙 乙

69七乙 乙一

6892 '

$60 \angle Z Z$

$8182 \mathrm{Z}$

$\downarrow \varepsilon 6 乙^{\prime} 乙$

990E'

เเ8乙 $\varepsilon$

$6882^{\circ} \varepsilon$

0G0 ' $\varepsilon$

$66 \varepsilon \Phi^{\circ} \varepsilon^{-}$

งเカレ:

$9 \angle t L \angle-$

06SIL-

OZ9L'L

ㄴEZ L

$\varepsilon \nabla \varepsilon Z^{\circ}\llcorner$

$9 レ \nabla Z L$

EStZ L

Z09Z' L

$008 Z^{\circ} \angle$

GE6 $\angle>$

Z8Zt L]

90Et $\angle]$

$\angle レ T V^{\circ} L$

8Z9t $L$

乙GS $L$

S09t $L$ -

089t L

6蚃 L

EG速 $L$

$9 \angle$ \&

LG\&9 L-

96ख9 $L$

69EL $L$

86敋' 2

QLEL $L-$

L政 L-

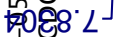

$5 \sum^{\infty}$

$\infty$

궝욤 
EZ90"GนL S†G9 GZL 98Z0.9ZL เ6Lて'9Zレ $\checkmark 0 \angle L \angle Z L$ UUG LZL$9809^{\circ} \angle Z L-$ $0089^{\circ}$ LZL 乙Z60.8ZL 66L8'レEL

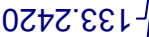
$\varepsilon 8 \angle G^{\circ} \nabla \varepsilon L$

99St・9ヤレ-

Z9L609L-
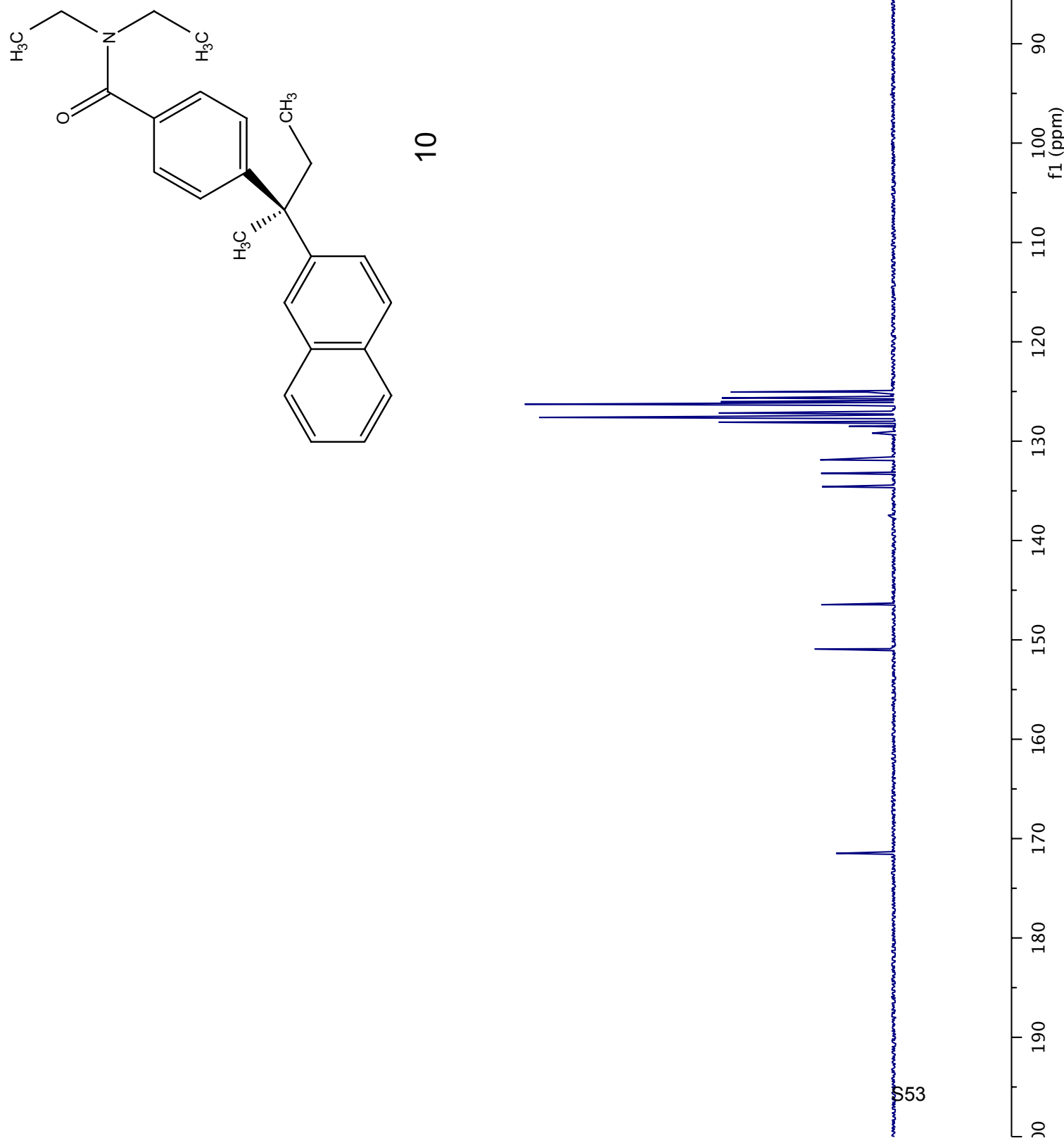
8GSL'0

$089 L^{\circ} 0$

E08L 0

EOZL'レ-

เ৪E乙

EOSZ 2$]$

0197乙

$0 \angle 9 Z 乙$

$\angle Z L Z$ Z

S6LZ

6†8Z 2

6เ6Zて

乙L6Z乙

6เOE'

乙๖०६て-

て†เと'て-

ง9ZE'

†868 ${ }^{\circ} \varepsilon-$

$980 \mathrm{~L}^{\circ} \mathrm{L}$

8ULL

OEZL $\angle$

192l'L-

हІว०ว 009Z L

0E6Z $\angle$

$\angle 96 Z^{\circ} \angle$

$666 Z^{\circ} L$

$8 \angle 0 E^{\circ} \angle$

8OLE $L$

$\angle \nabla L E$ L

$\nabla 6 \varepsilon \nabla^{\circ} L$

LレVT L

OLGT L

EESt $L$

6E9t $L$

$999 t^{\circ} L$

$\angle O L T^{\circ} \angle$

¿E $\angle \nabla^{\circ} \angle-$

6เ8t L-

0†8 ${ }^{\circ} L$

1987 L

†G6t L

$\angle L 6 D^{\circ} L$

$\stackrel{\infty}{\sim}$

$\varepsilon 989^{\circ} L$

$800 \angle L$

GLLL L

$608 L^{\circ} L$

$676 L$

OLZ8 L

SEE8 $\angle$

19E8 L-

$\angle 826^{\circ} \mathrm{L}$

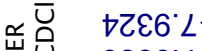

9GE6 $L$

GE七6 $2-$

S9t6 $\angle$

$\rightarrow 096^{\circ} L$
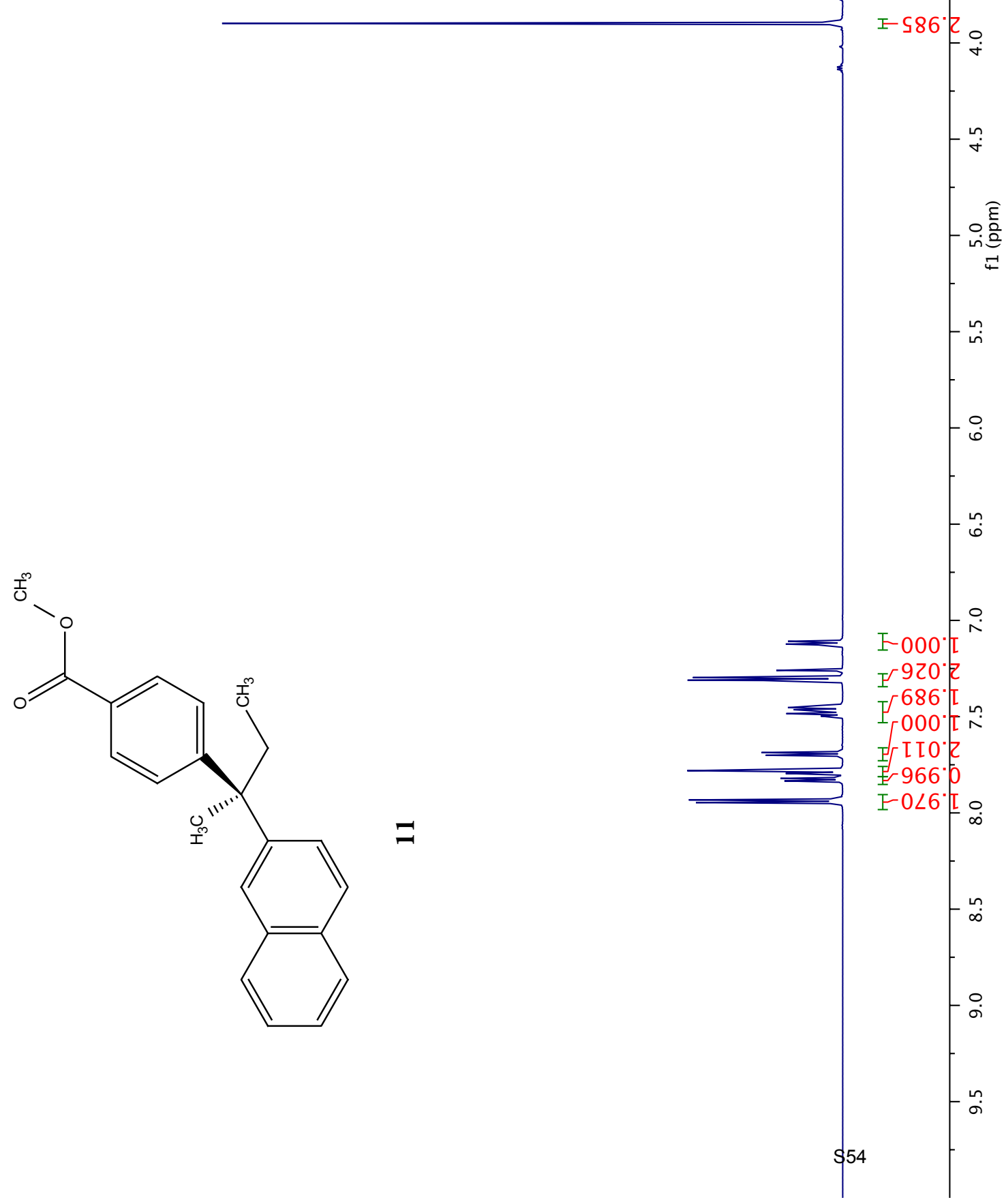
$98 Z \angle \angle Z L]$

6IGL'LZL

L乙७8 LZL-

ง6ฑレ`งレー

Z6乙Z $\angle 9 レ-$

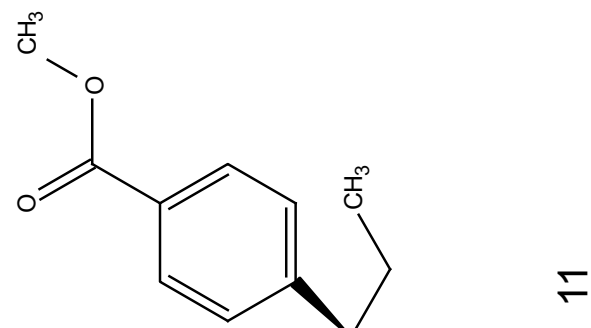

ن্ল 

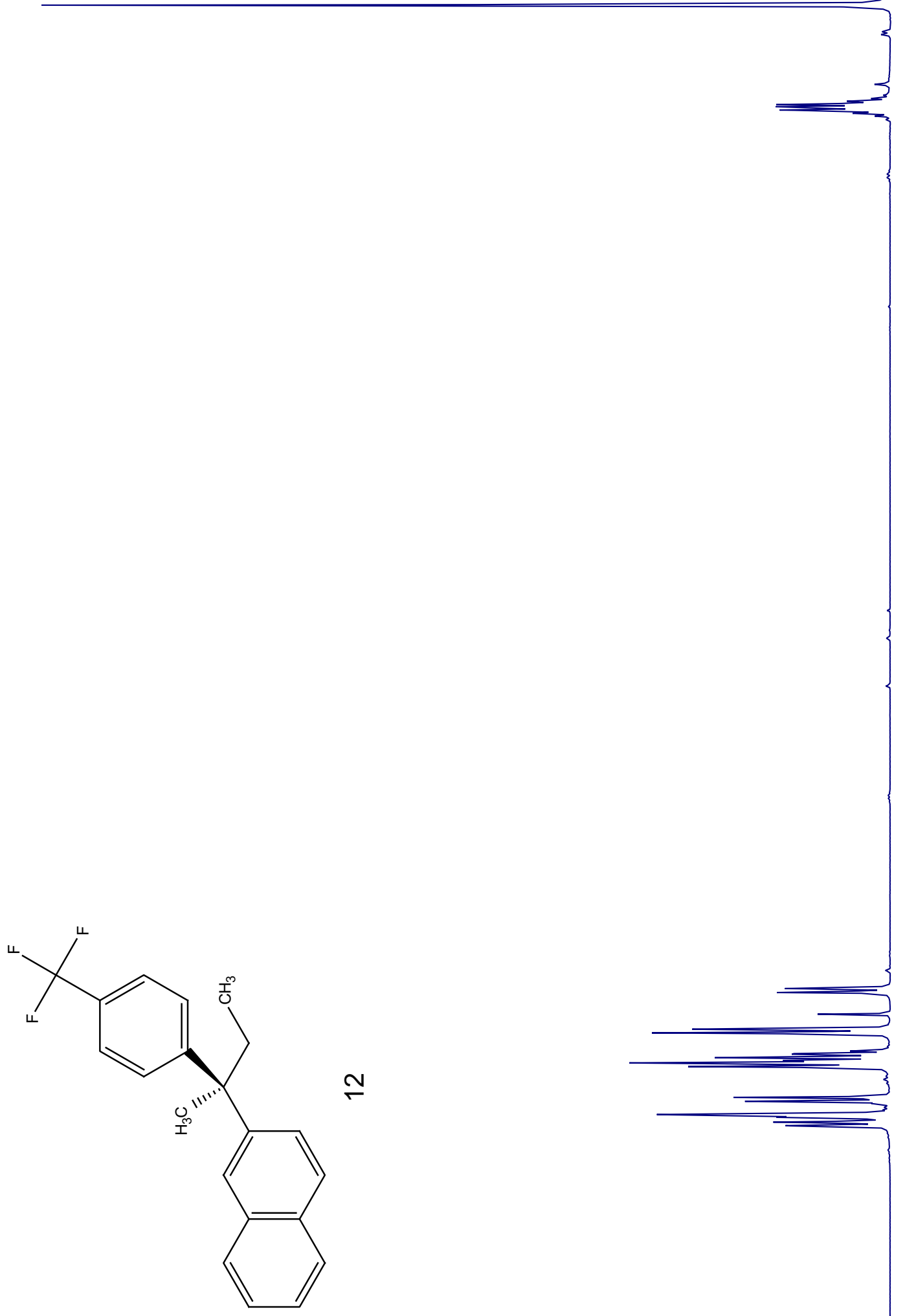


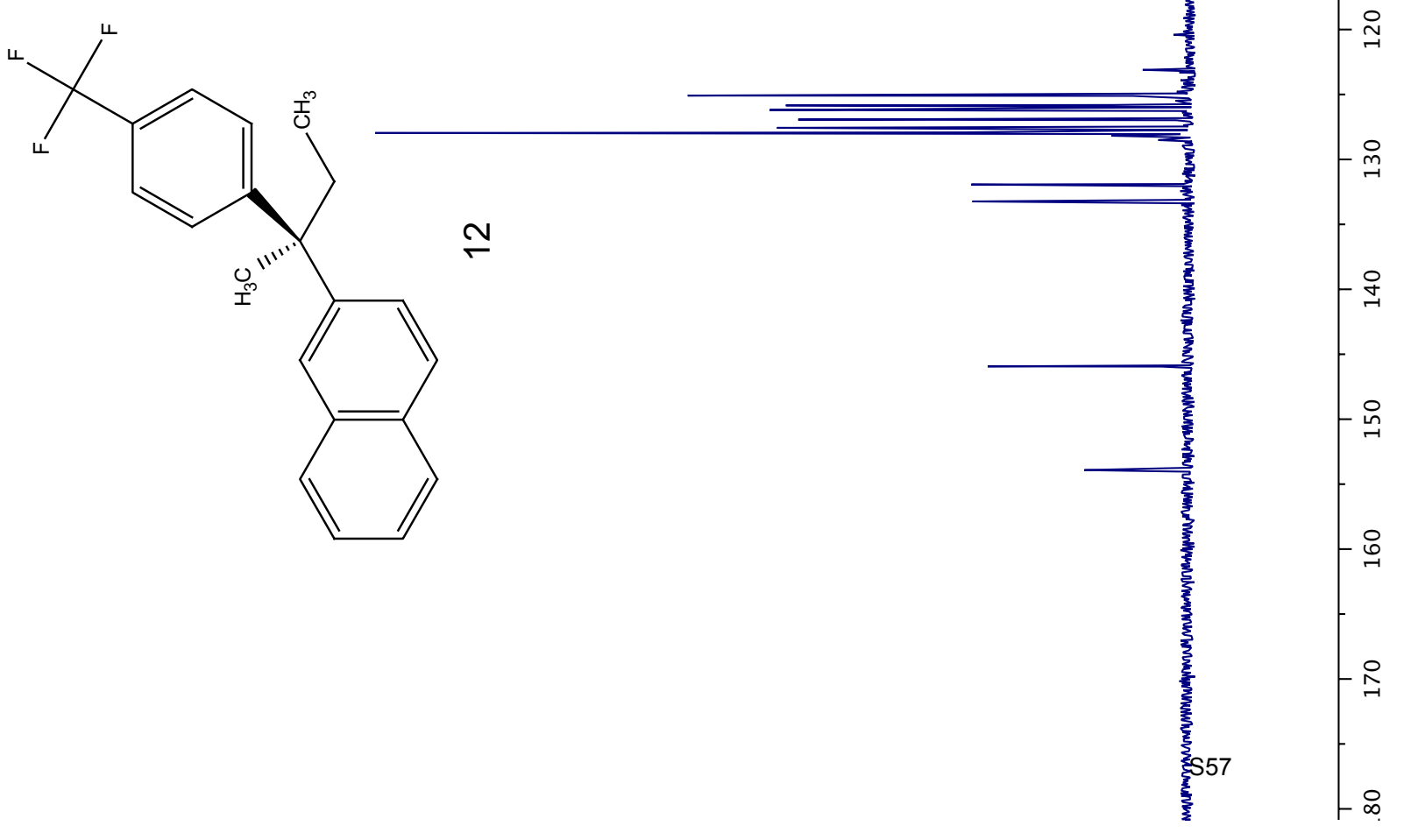




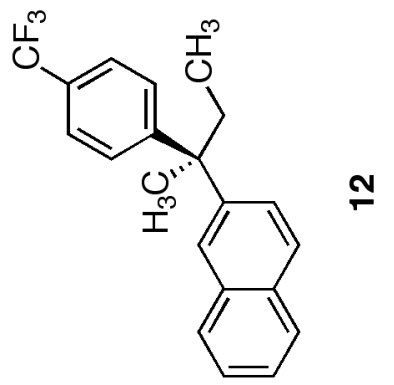


L6SLO

OZLLOO

Z† $8 L^{\circ} 0^{-}$

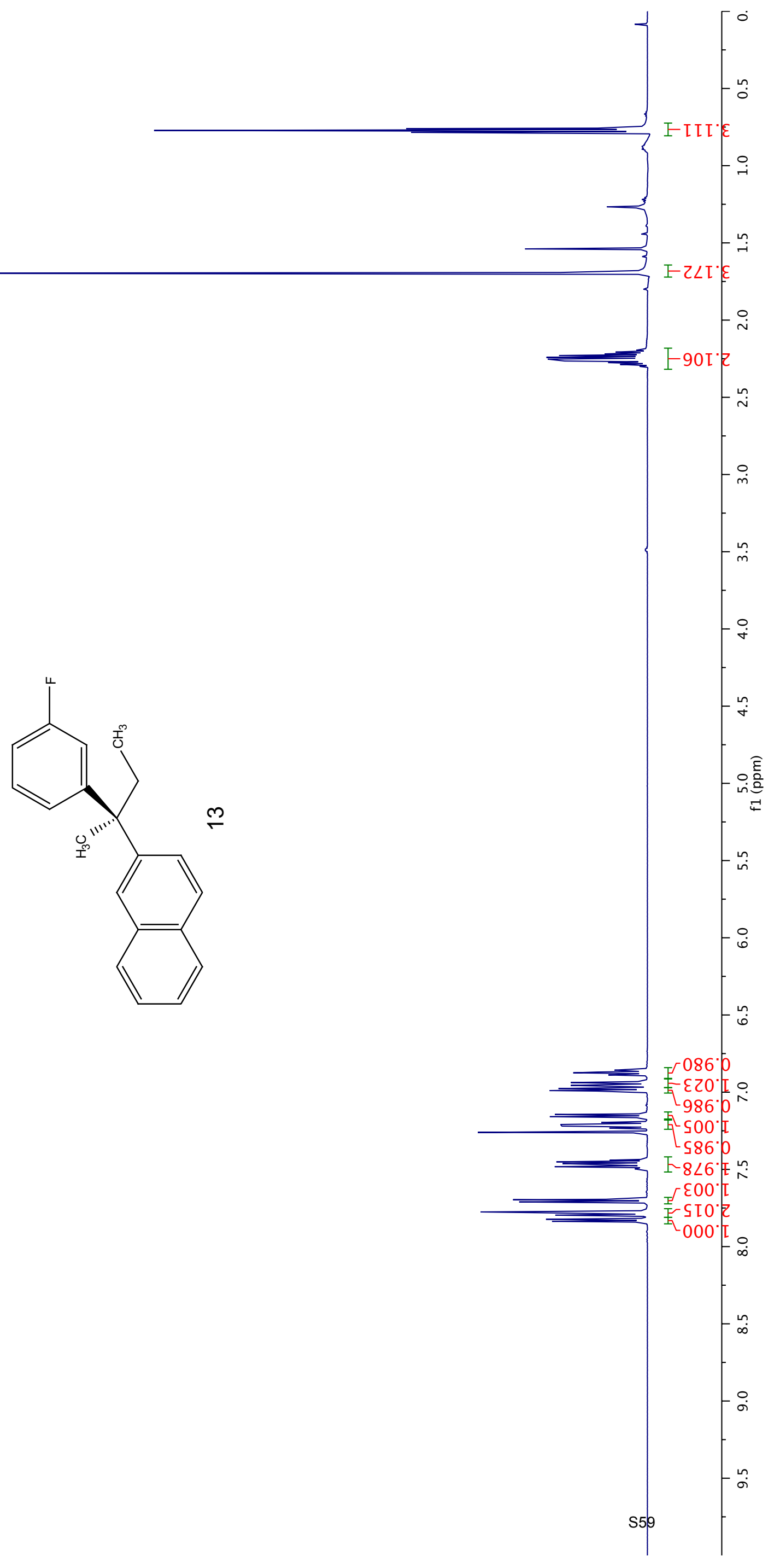


Lo\&8.92-

0ง๋8 $\varepsilon \varepsilon-$

9ᄂ $\angle 8^{\circ} 97$

$8188^{\circ} 97$

ELGS'ZルL ZL69'Zル ZGZ9"ナル เ69L・レレ $\left.9 G \angle \varepsilon^{\circ} \varepsilon Z L\right]$

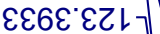
$6086^{\circ} \mathrm{DL}$ †LEL'GZL $\checkmark 680^{\circ} 9$ Z $]$ $\angle \varepsilon 86^{\circ} 9 Z \mathrm{~L}$ 8StS LZL†S08 LZL-

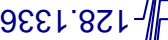
LGLE'6ZL86Zt6てい \&9E6 LEL

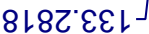
6เ8Z'9ヤレー

8889'ZSL ELE9'ZGL
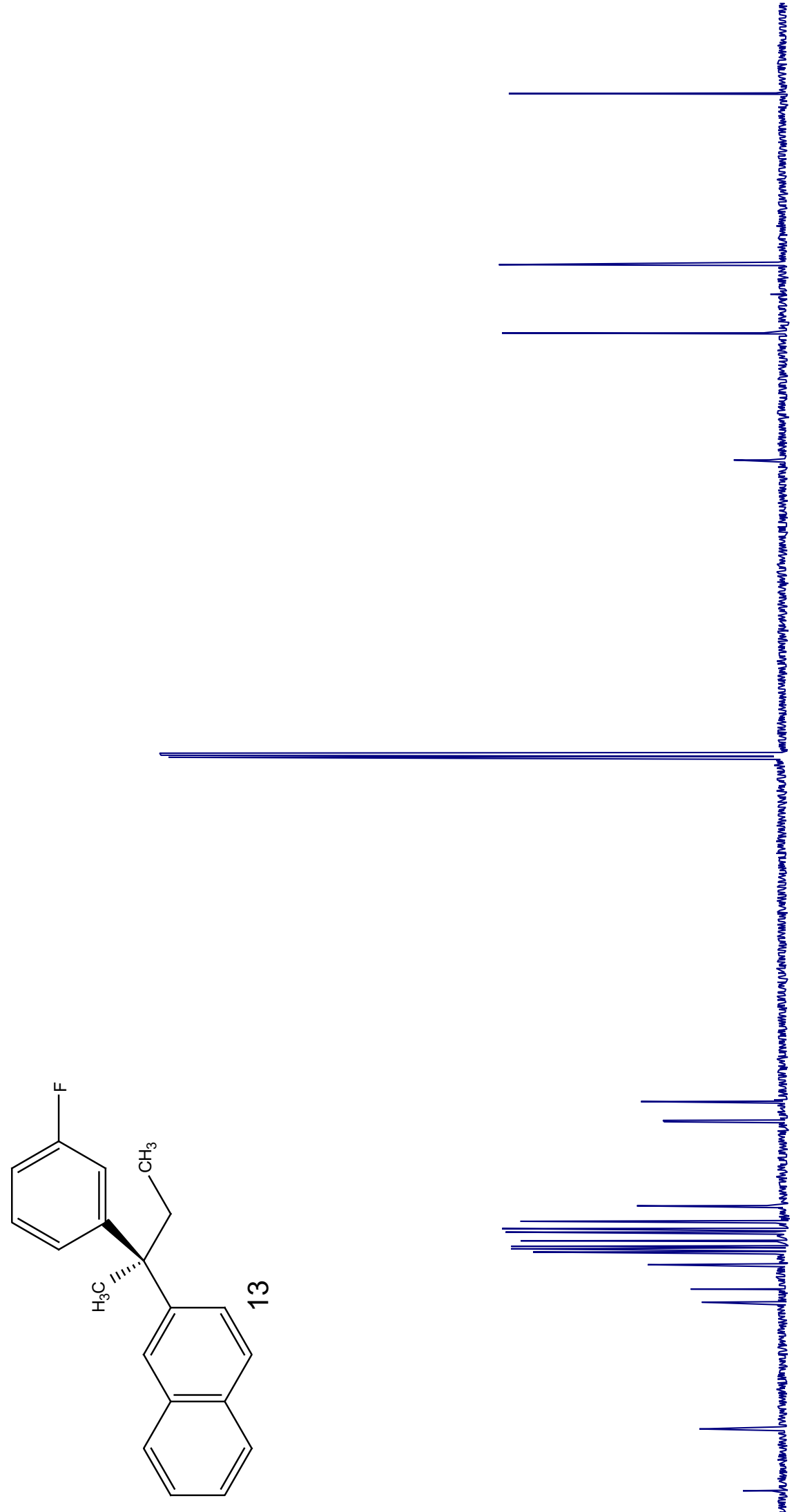


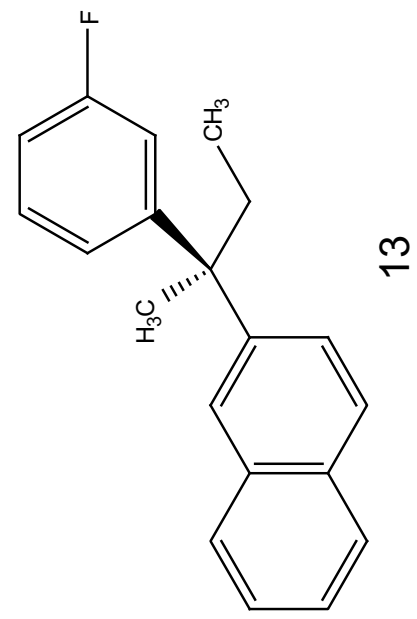


9ع9L 0

8SLL'O-

$088 L^{\circ} 0^{-}$

OE9l'Z

ZGLL'Z]

09812

$\checkmark \angle 8 L^{\circ} Z$

9L6L' 2

L60Z ¿

LSLZZ

0ZZZ Z

6L乙Z Z

$00 \downarrow 乙 乙-$

LOSZ 2

งZ9乙乙-

† 乙 乙

$\angle \forall \angle Z^{\circ}$
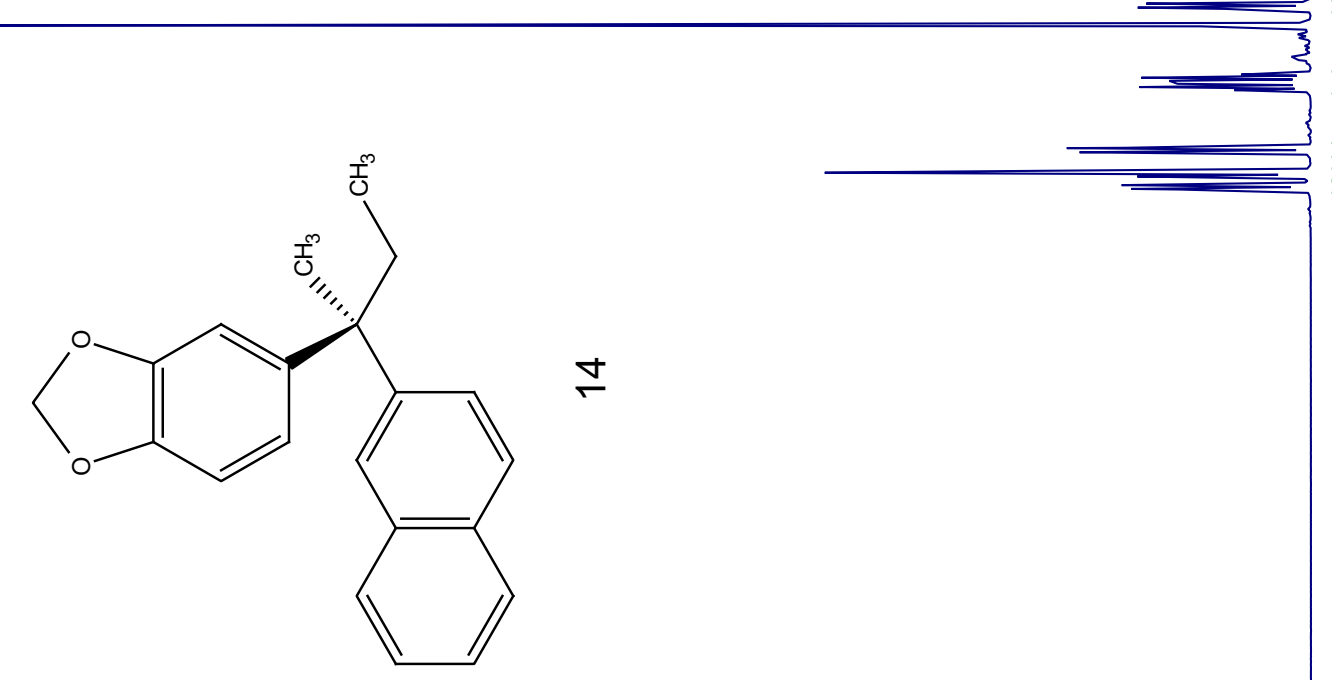
9L9ะ 6 -

DZOL:LZ-

$\varepsilon เ 90^{\circ} \downarrow \varepsilon-$

ᄂLE9.9t-

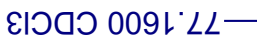

0LE6.00L-

$1609^{\circ} \mathrm{LOL}$

S09980L-

069ع'0ZL

GSGL'†ZL

$\checkmark \angle 89^{\circ} G Z$ ]

G७86.9ZL]

89tレLZL

IELGLZL-T

ᄂ $199^{\circ} \angle Z L-$

980เ'8ZL][

†998'レEL

068乙 $\varepsilon \varepsilon\llcorner$

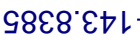

て8t7・Sカレ

$8760^{\circ}$ Lヤレ-

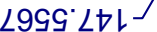

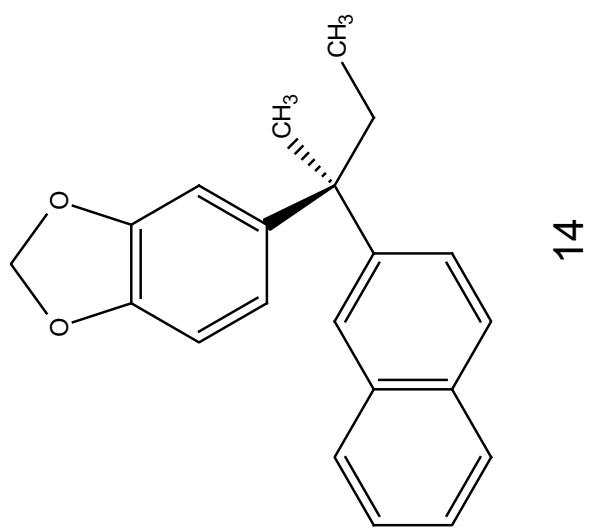


$26 \angle 9^{\circ}$

$\frac{m}{U}$

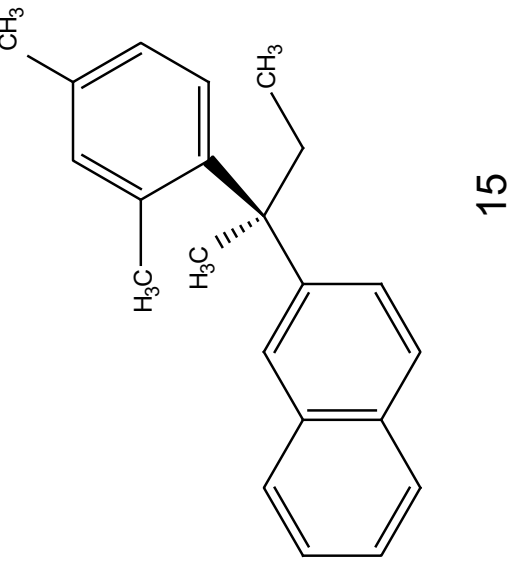

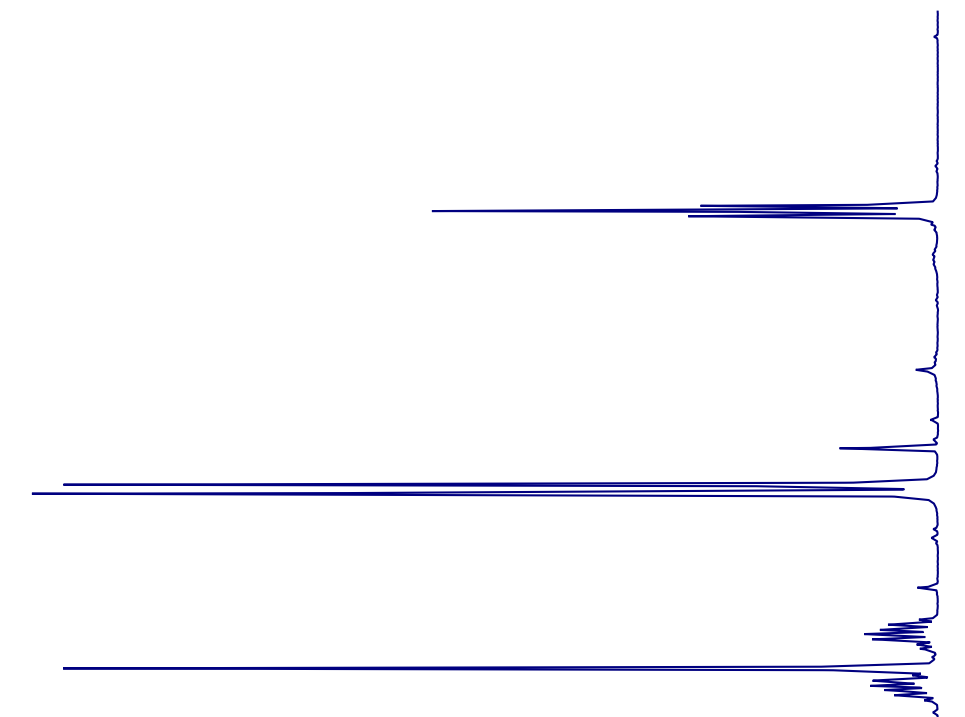


$92 \angle 8.02=$

sl88 l一

$19 \angle 6 \angle 2-$

8Zเ8'Zะ-

8ะง8.97-

GZ8L •ZL

GELZ GZL -

90\&8 GZL

\&960"9ZL

GZ8Z'9ZL

$886 t^{\circ}$ LZL

$\left.\angle O S G^{\circ} \angle Z L\right]$

S80L LZL]

9Z108ZL

$\varepsilon \varepsilon 0{ }^{\circ} L \varepsilon L$

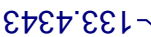

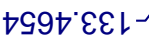

Z8tL $\angle E L$ -

$8 \varepsilon \angle L \angle E L$

เレタடてャレー

6เ8เ・8カレー
Iำ

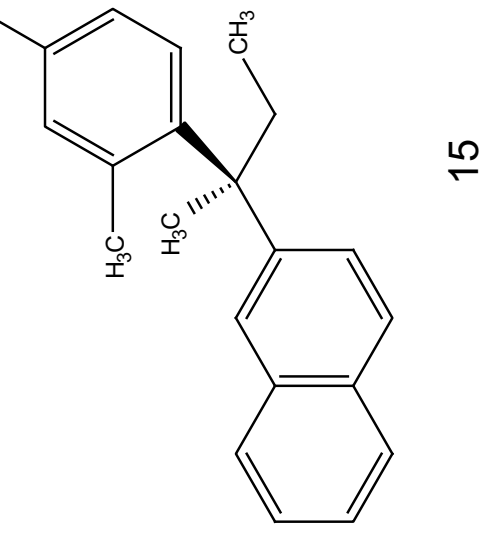

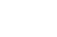

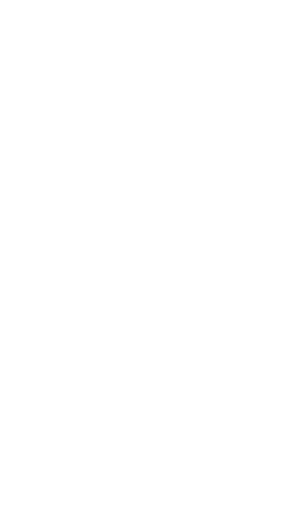

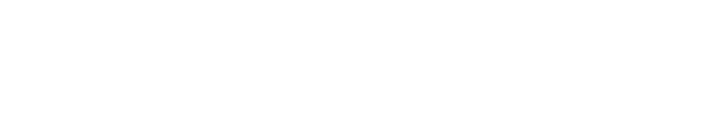
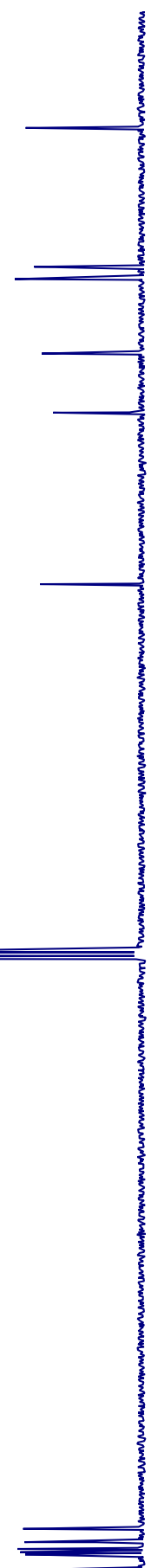
OLSL'O

E69L'0

SI8LO-

ع089レ-

$0 \angle 8 \mathrm{~L}^{\prime} \mathrm{Z}$

Z66l'Z

เOLZ]

乙Lレてて

งเZZZ

86Z乙 $て-$

LEEZ $冗$

$\varepsilon 乙 \triangleright 乙 \succ$

†๑佂

乙२९乙て-

†๑८ 乙-

9†9Z乙-

89L乙 乙

G8จL'E-

เยเ6 $\varepsilon-$

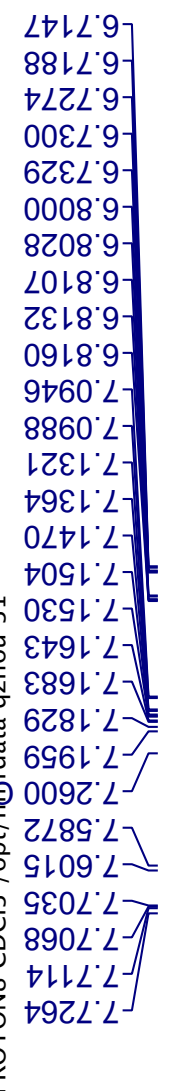

$\underline{\square}$
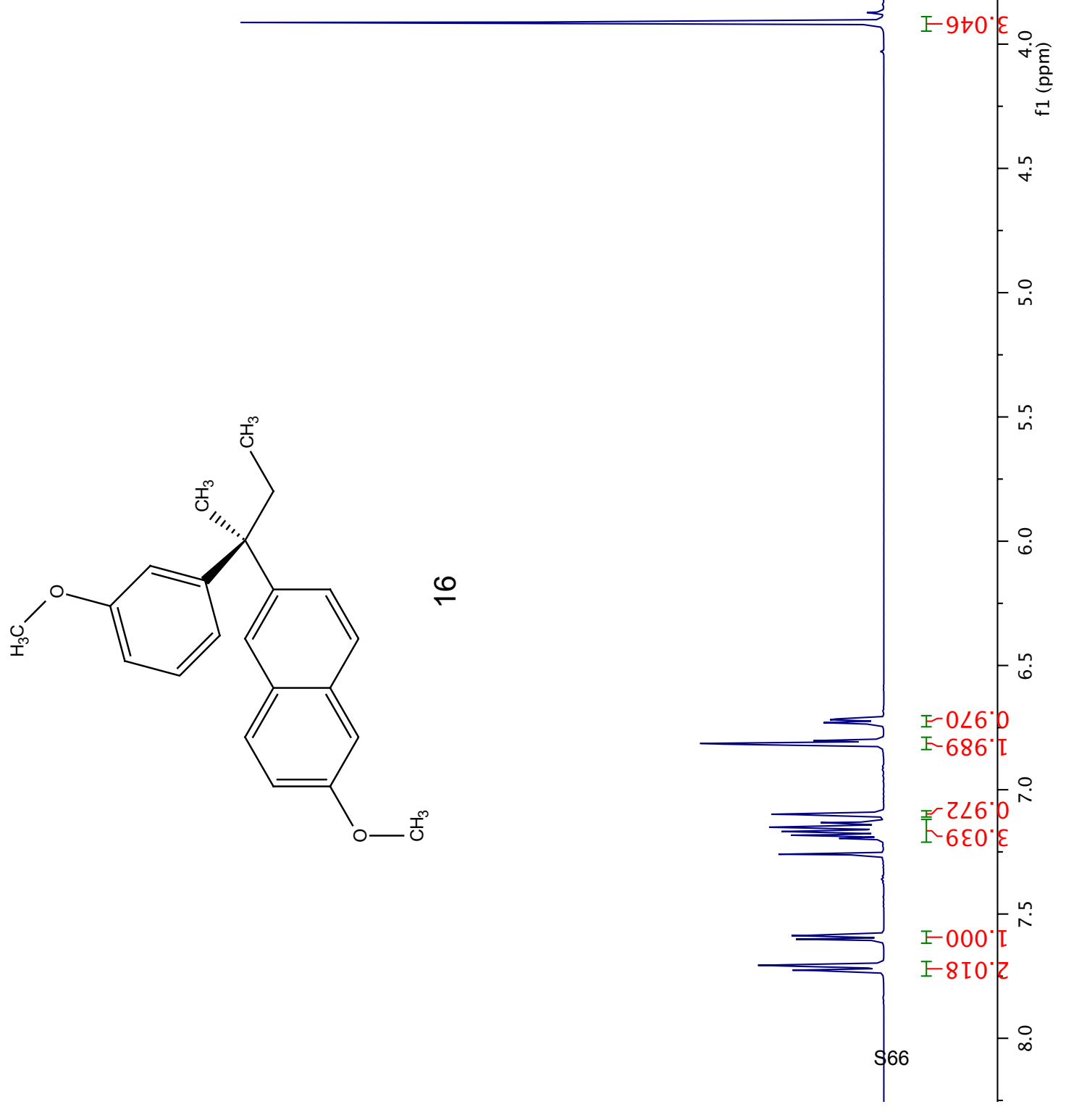
ฤ6เ゚ャレレー
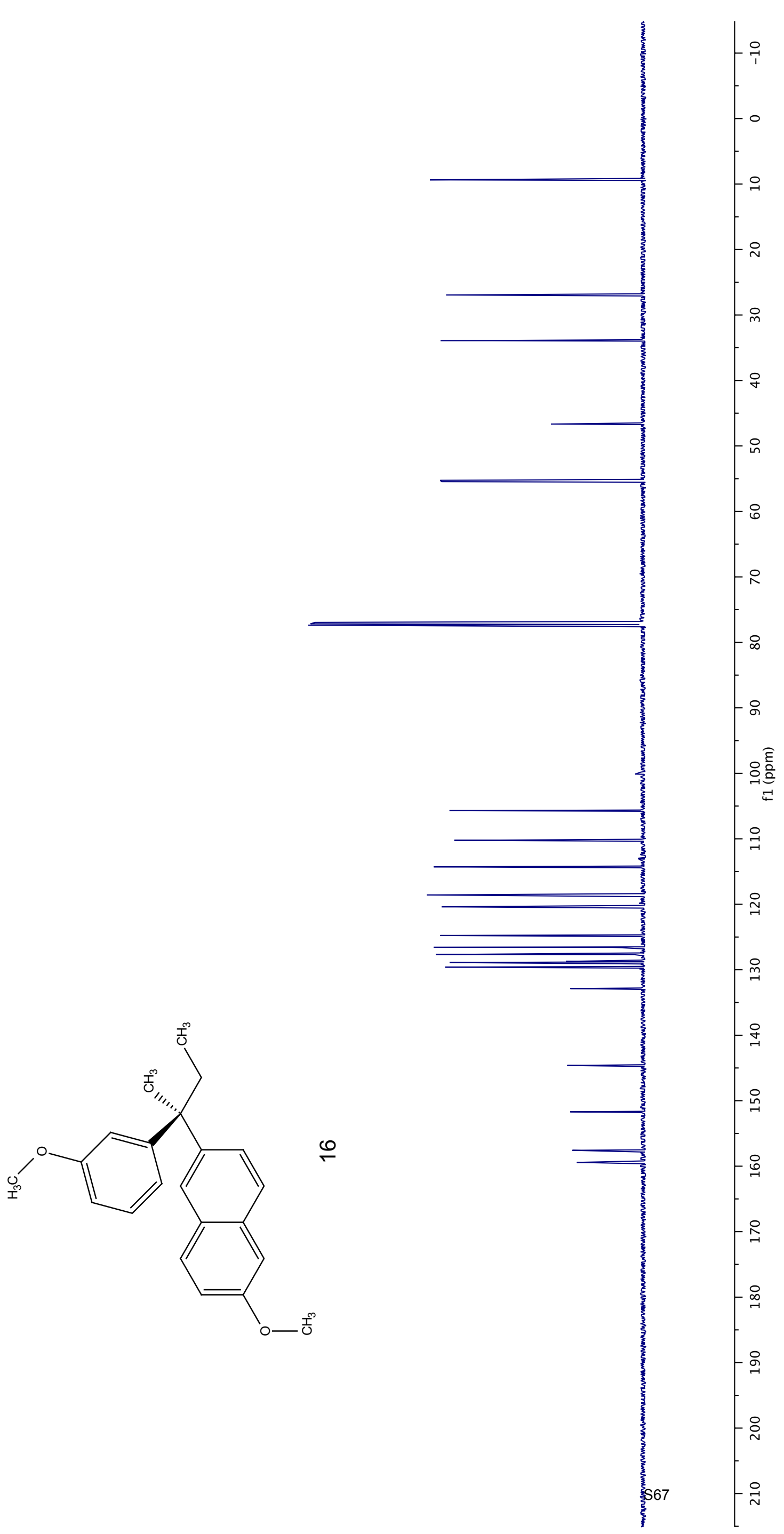

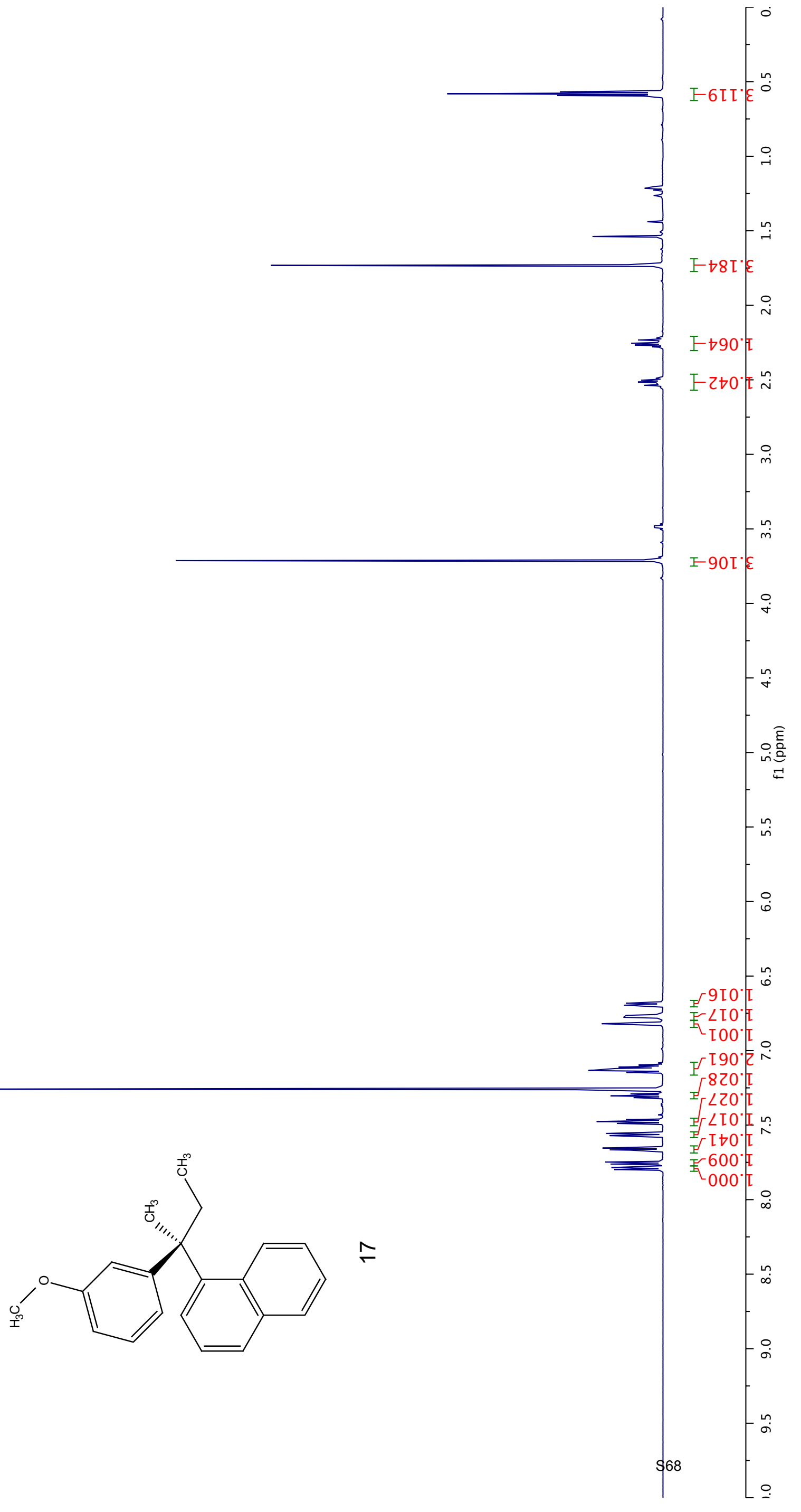
8†ยt 6 -

06عt $62-$

$\varepsilon 0 \angle \mathcal{G}^{\circ} \varepsilon \varepsilon-$

$0 \succ 9 t^{\circ} \angle t-$

OZLZGS-

EІ०هว 009l LL—

8L96 $60 L-$

Lยเレยレー

6988.6レー Z9L9' $\downarrow Z L$ เ69L'十ZL-

$\varepsilon \varepsilon 68^{\circ} \nabla Z L-F$

6†เย'งZL

$0+6 Z \angle Z L$

$9 Z \angle 8^{\circ} \angle Z L$

6EZ0 6ZL

99LZ'6ZL-

O†69' $L E L$

เ6ع0 ตEL

เ66乙๕もレ

9960๕ระー

9เ8969เ-
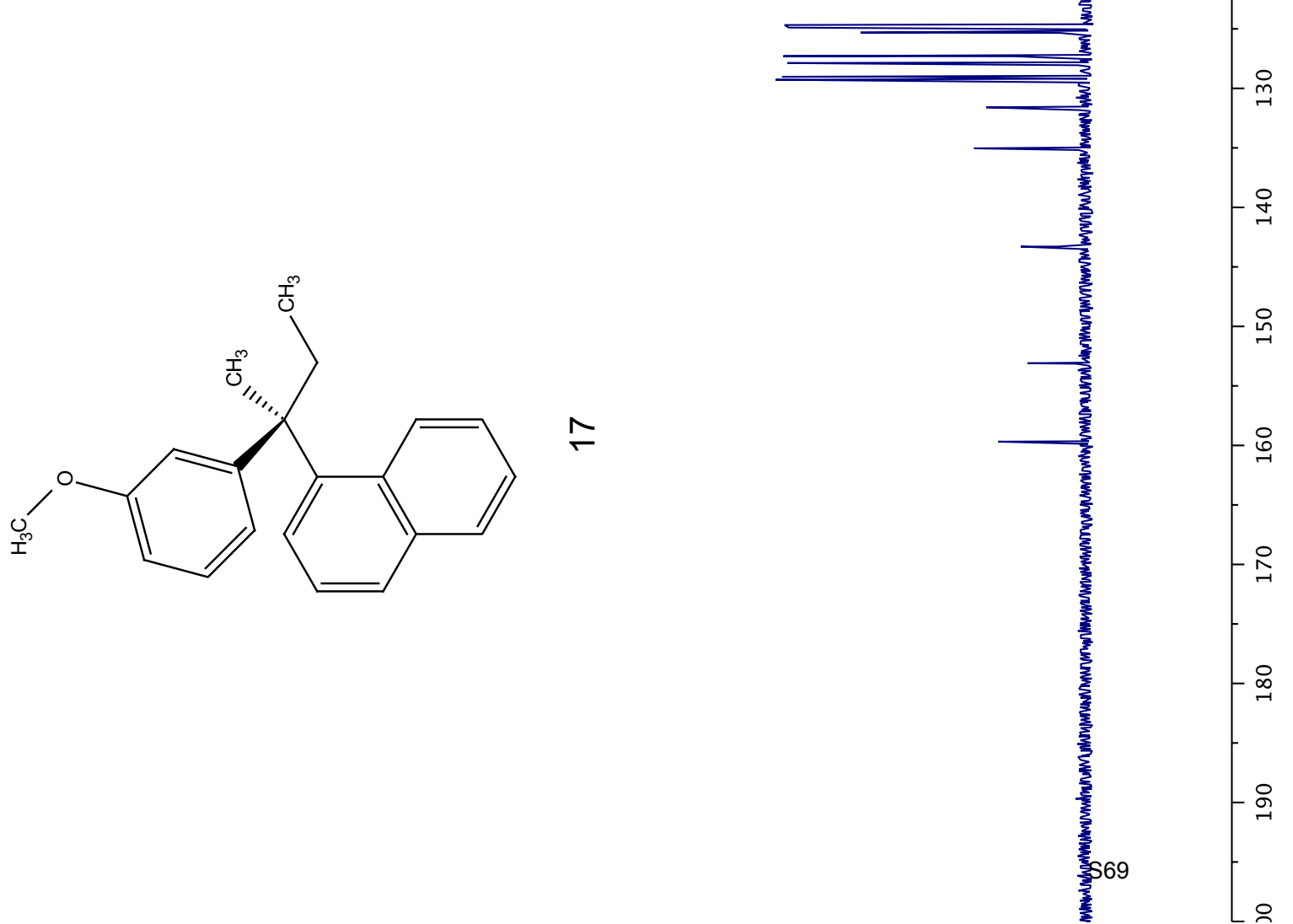
$\angle S L \angle 9$ SLLL'986L L'9$\angle L Z L 9$ 乙OEL 9 GE\&L'9乙GEL 9 ESLL'9 Z8LL'9] ᄂZ8L 9 6E6L 9 $\mathrm{G} 6 L^{\prime} 9$ 696L'9†6 L 9 $\angle Z \angle L^{\circ} \angle$ 6981'L $066 L^{\circ} \mathrm{L}$ EES $L \perp=$ †09Z L $\varepsilon \angle 9 Z^{\circ} \angle$ Z06E $L-$ $8 \varepsilon 6 \varepsilon^{\circ} L-$ 6†0七 LG8Rt $L$ $\angle E 29^{\circ} \angle-$ ZLE $9^{\circ} \angle$ $0 t 98$ $\angle 88^{\circ} \angle$ 6862 82 0 : 8

응

ำ

บิ

$\stackrel{\infty}{\rightarrow}$

เ่

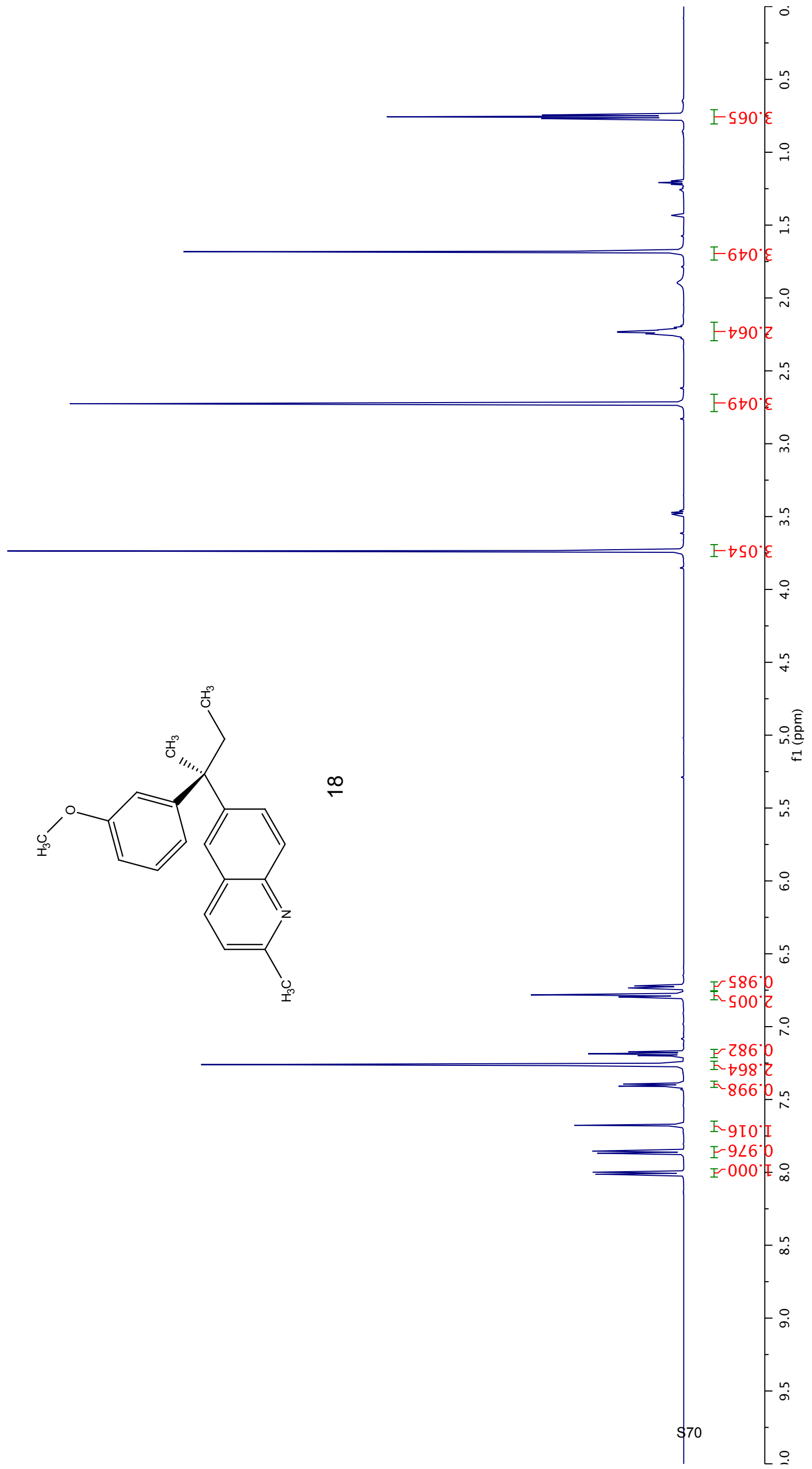


†รเE. 6 -

696E'G乙-

9868:9乙-

乙ะ06.ยะ-

8GSL $97-$

98\&ح' $99-$

ยเวดว 009 : LL_-

69Lยㅇㄴ-

ง86でャルー

て๖9て・0てレ

LOZ0ZZL

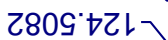

\&280.9ZL

28928Zレ

†8เ0 6乙レ

SLL9.0EL

m $0 \angle 9 \varepsilon \cdot 9 \varepsilon L-$
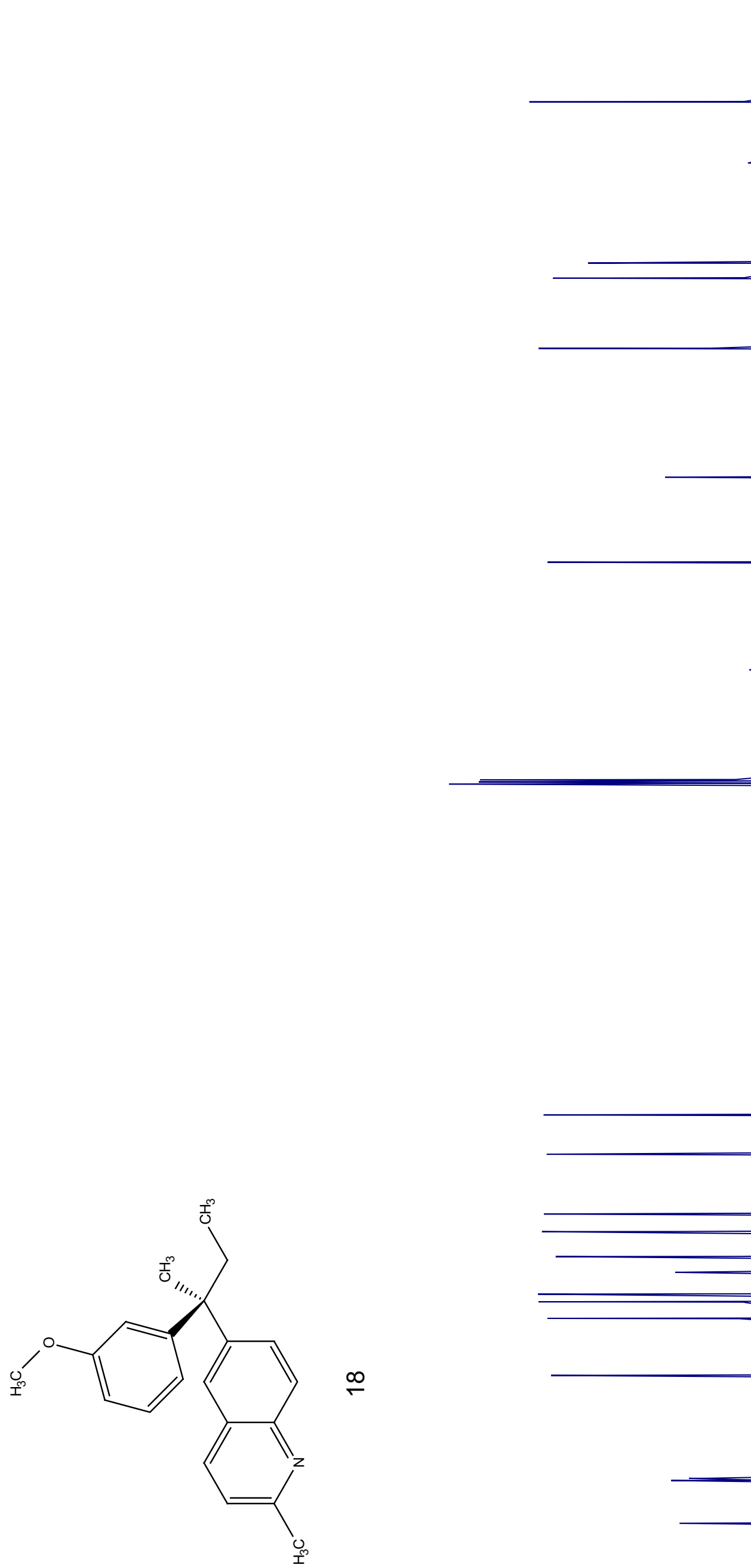

- 0

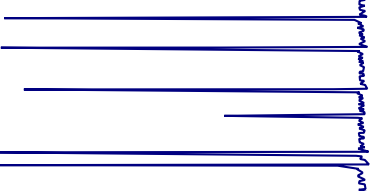




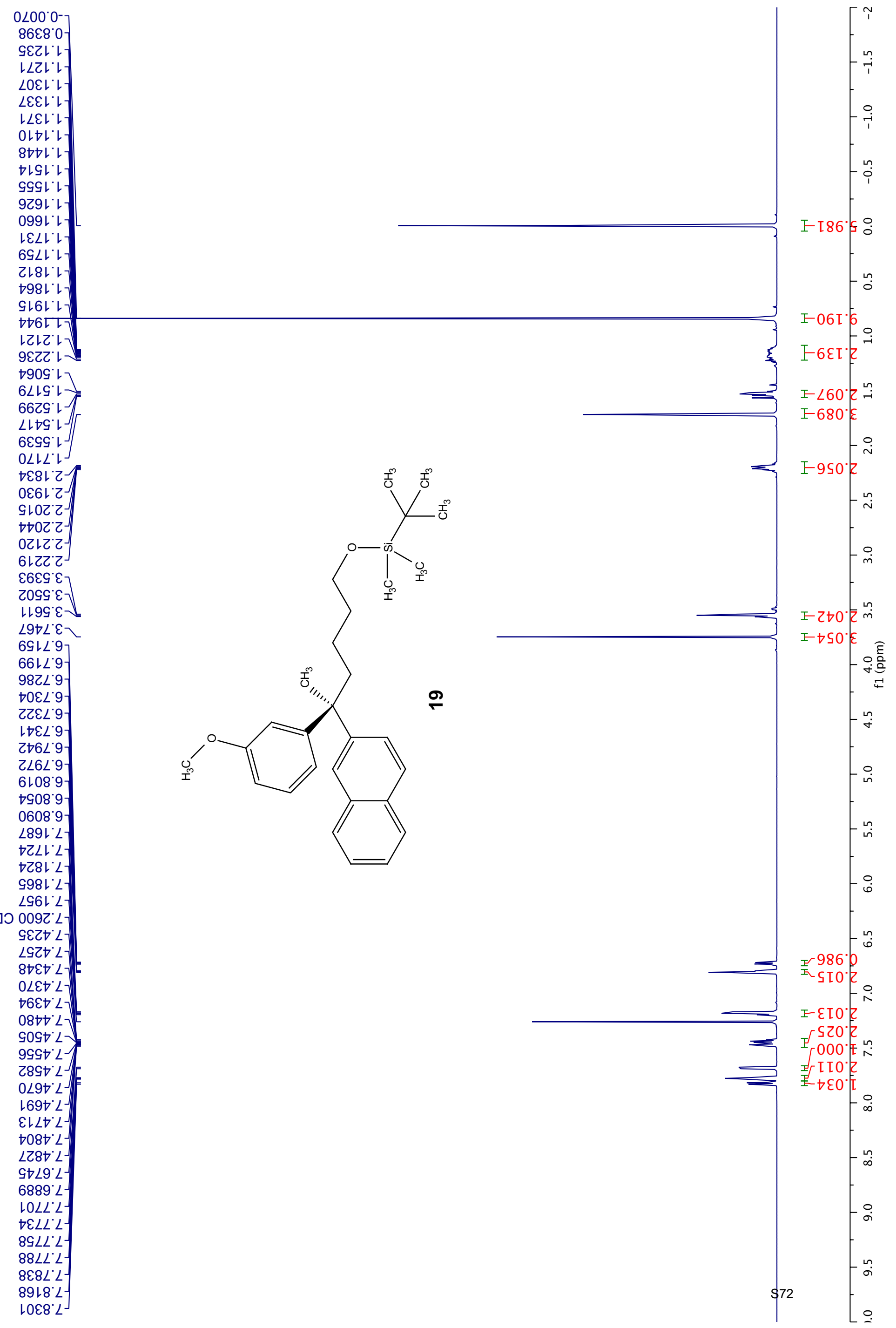


ட8เナー8レ-

1082レー L990.9Z

$\triangle \nabla \angle 9^{\circ} \angle Z=$

L乙69' $\varepsilon \varepsilon-$

乙๖6ย゙レーー

ง8เ9:9t-

6カฤでง -

$\angle 9 \angle L^{\circ} 89-$

98820レー

LEレでャレー

$\checkmark 88 Z^{\circ} 0 Z$ ᄂ

9レナLナてレ

L8EG'GZL]

乙LZ6.GZL

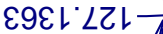

¿†09' LZL-f

0† $99^{\circ} \angle Z L-[$

6ZعL'8ZL-

†896'8Zレ -

$6968^{\circ} L \varepsilon L-$

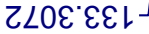

LOLO LヤI-

OE8G'เGLー

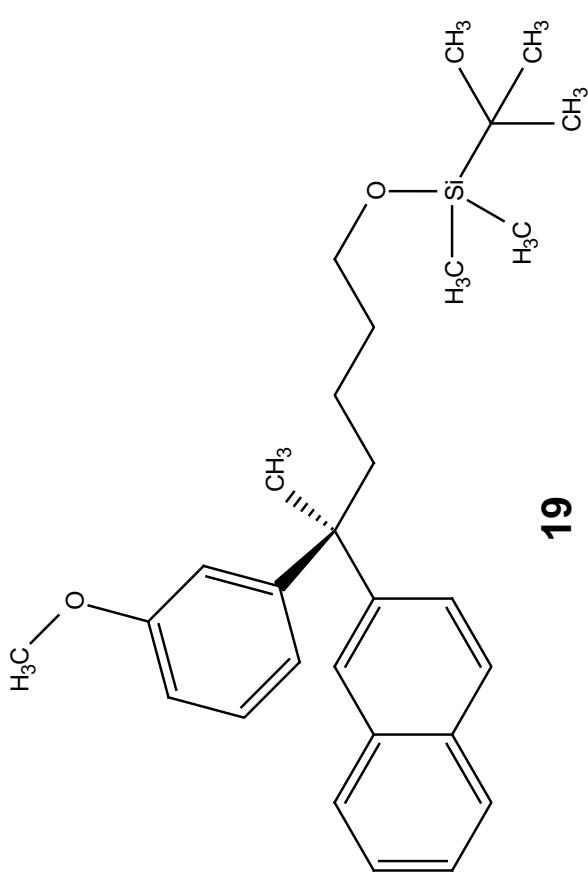

r 


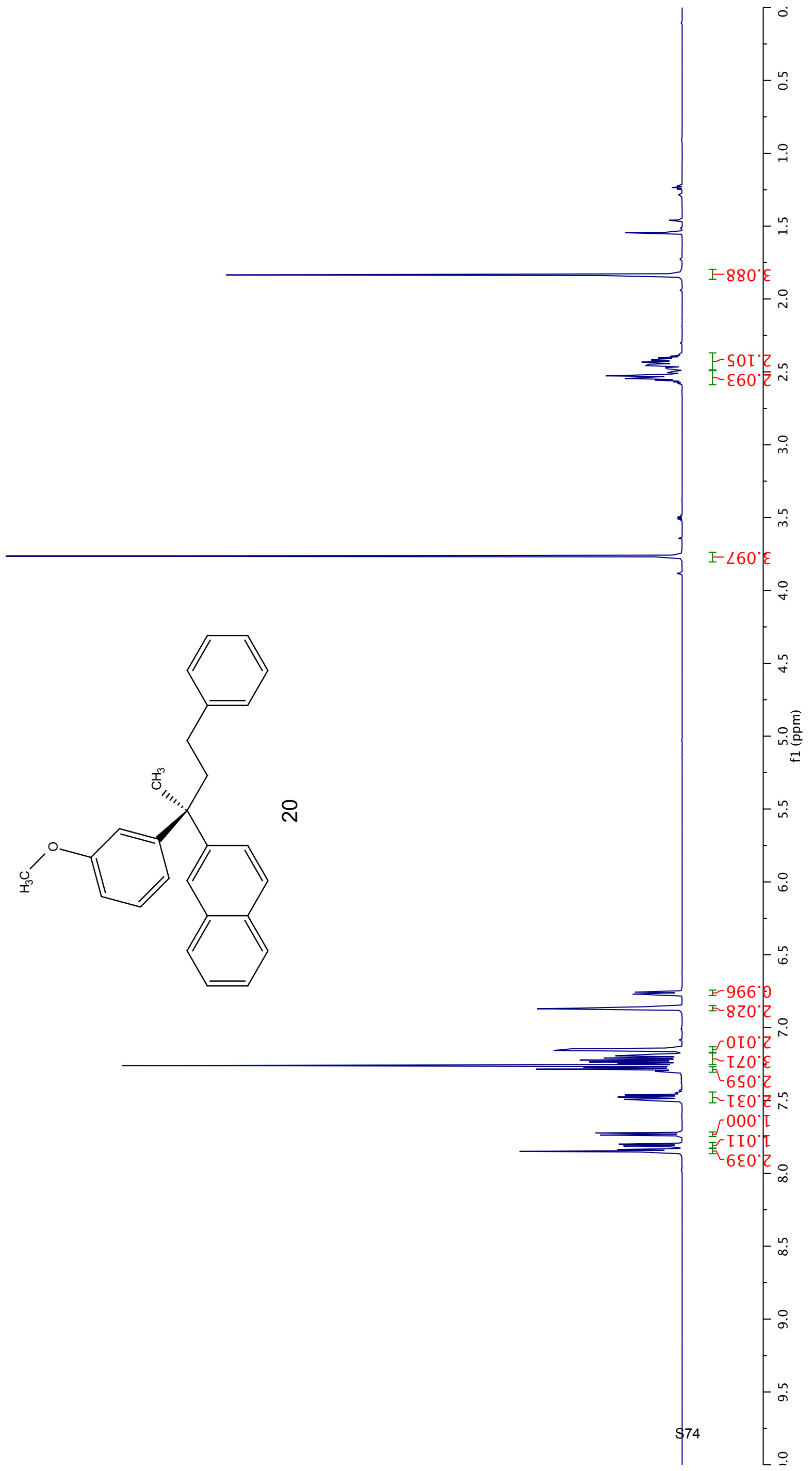




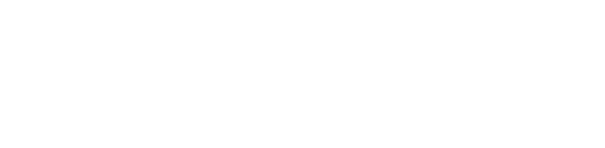




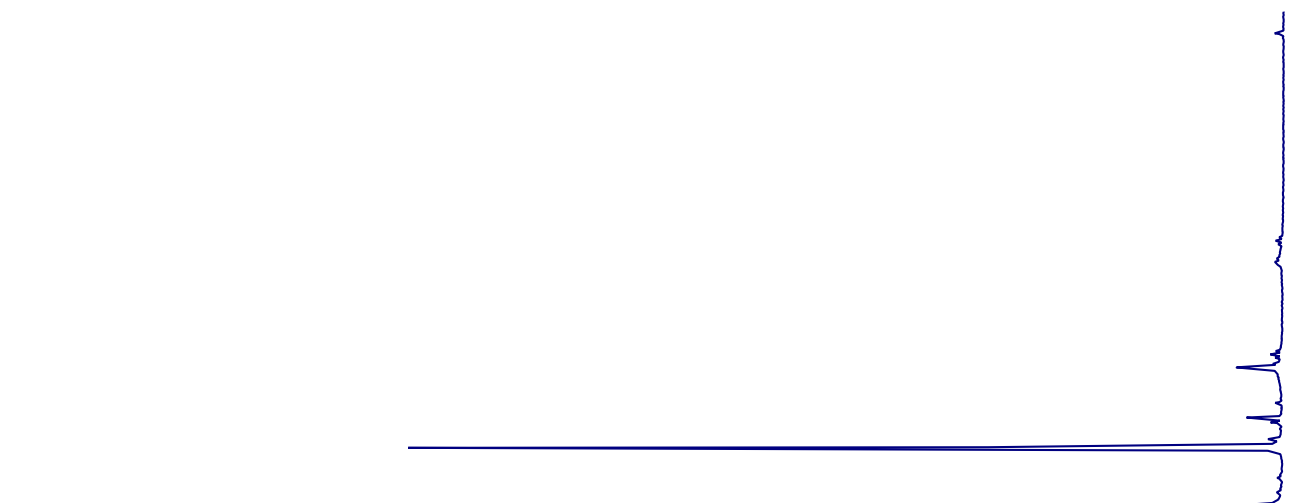

OtEt $L$

ยEtt $L$

$\angle S \nabla D^{\circ} \angle-$

$6 \angle \nabla t \angle$

6SGD L

†8St L

$019 t^{\circ} L$

$\stackrel{n}{\sim}$

G69t $L$

†レ $\angle \forall ' \angle$

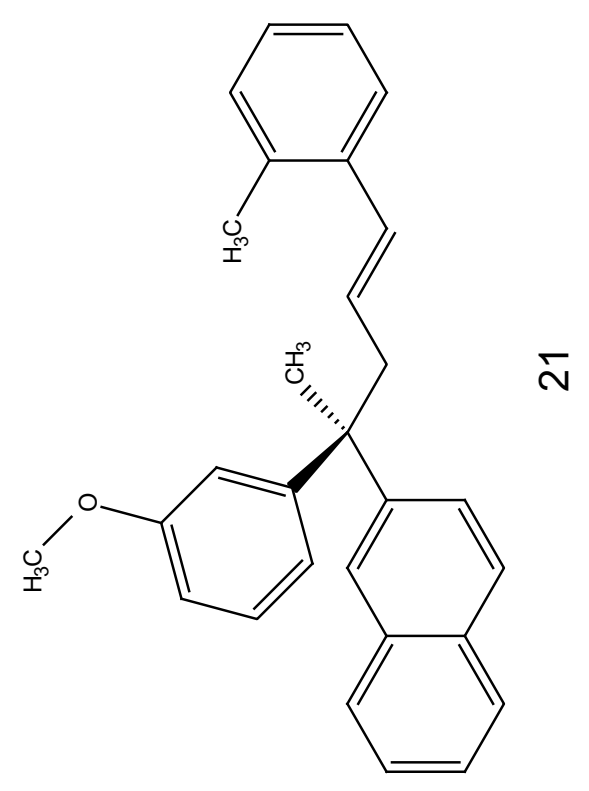

$\bar{N}$ 
งเE9ㅇㄴ

ย6乙でャル 809Z0ZL $\angle 9 Z 8^{\circ} \triangleright Z L$ $\angle 6 \angle 9^{\circ}$ GZL 9L06.9ZL 0†Z0.9ZL $9 \triangleright \nabla 0.9 Z L$ $\angle L \nabla 0^{\circ} \angle Z L$ GOOL LZL OLEG' $\angle Z L$ L08L LZL 6เ6レ8Zレ てヤ6ฑ 8てレ †9606乙L

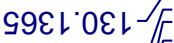
ย6เレเレL†686เยL

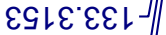
$\neg Z 0 L ' G E L$

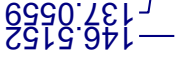
0896.09レLIEG 6G -

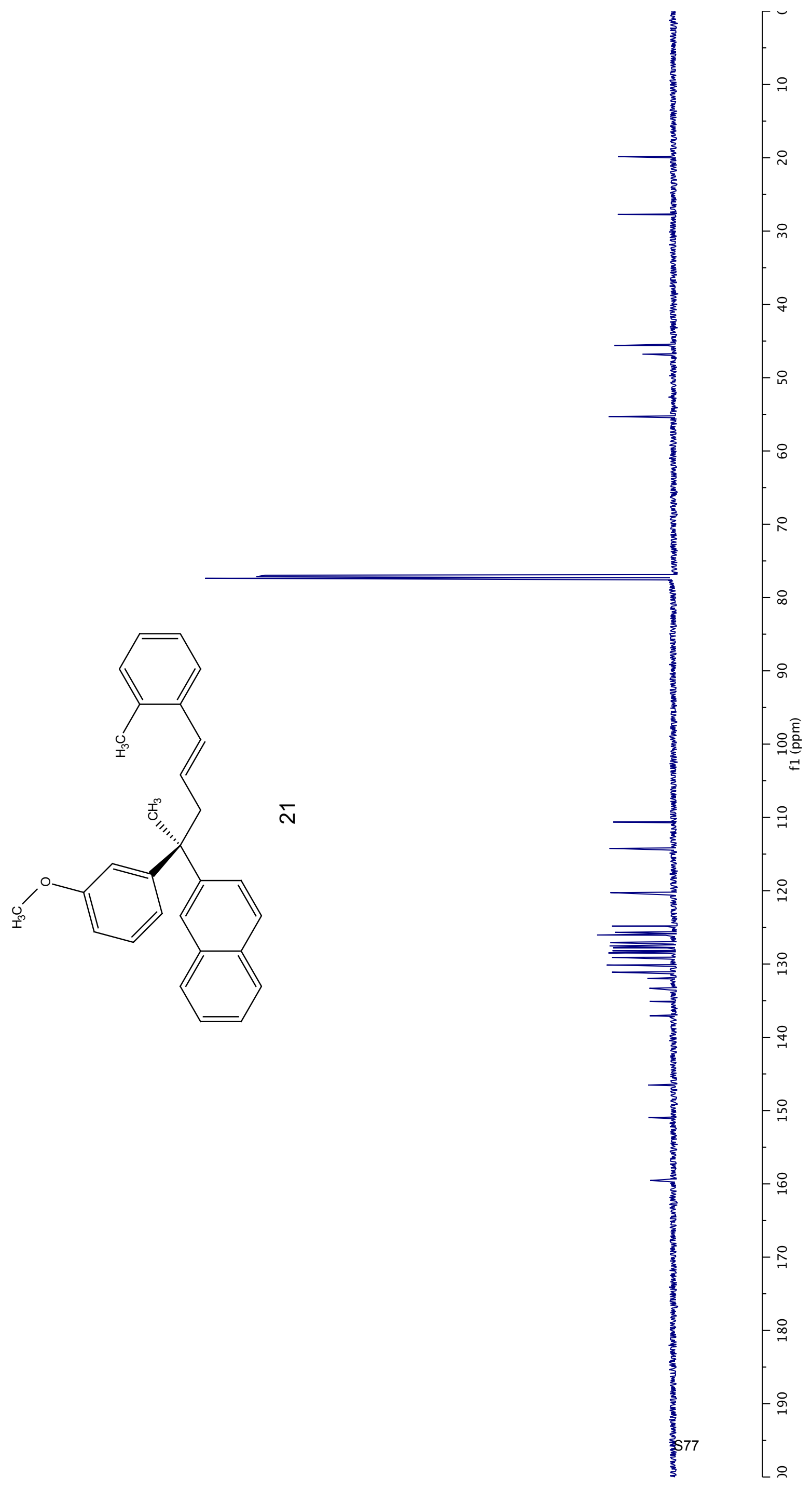




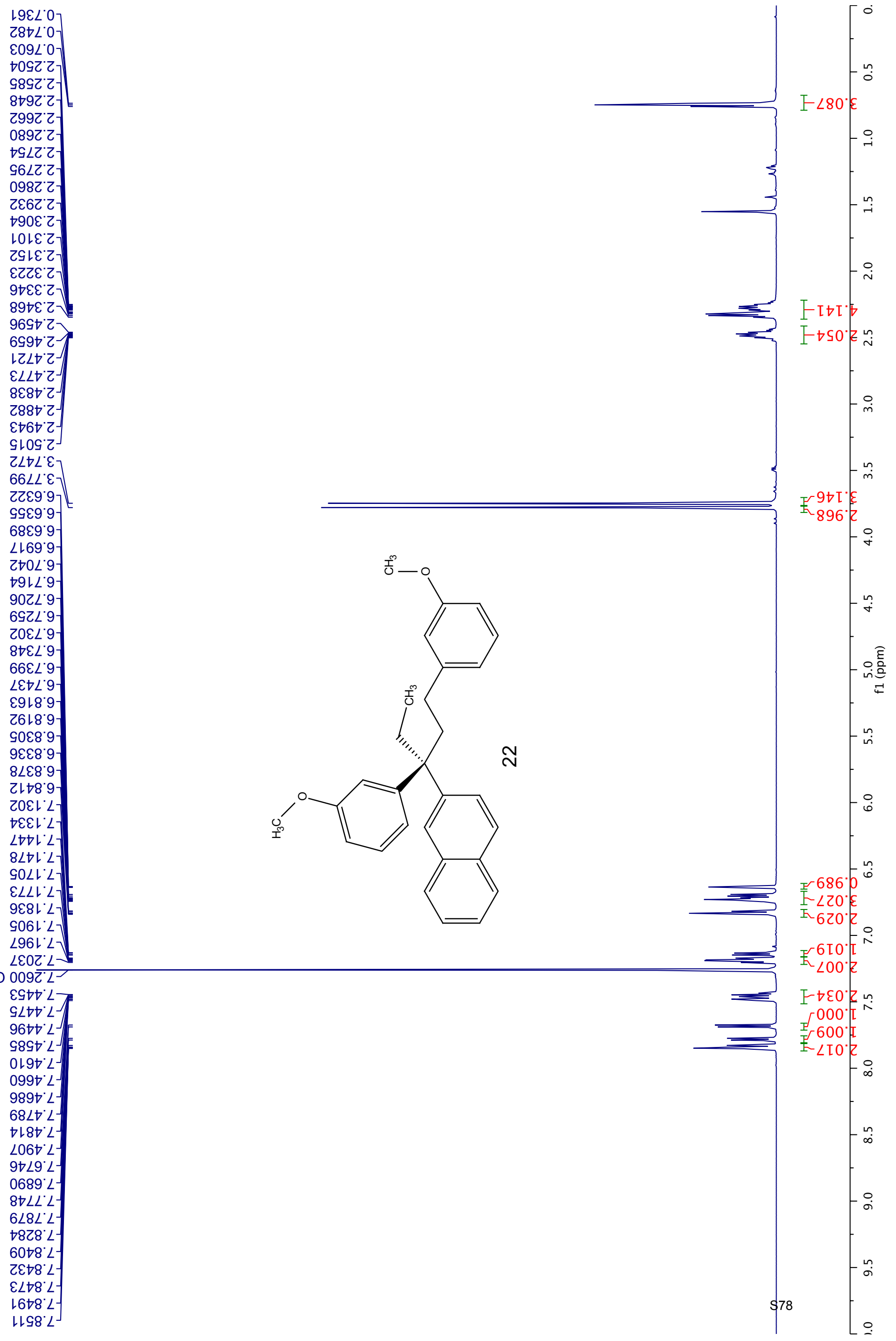


98Z9.6Z -

0เ88.0 -

0เE6.8\&-

ᄂ $\angle 866^{\circ} 67-$

628Z 99

ع982 $9 \mathcal{G}^{\prime}$

ยІวดว 009 : LL—

$6 \angle L \varepsilon^{\circ} 0$ เᄂ OL86.0

89レナナル

90L8ヤルレ

$88 Z 6^{\circ} 0$ L

LEE6 0Zレ]

9967 GZL

6LE9 G乙レ

†เ86.9ZL]

†৪ヤ LCL]

6ZIG'LZL

レナELடZレ-F

ยレLレ8Zレ

8L06.8ZL

EOSt 6ZL

80\&6. LEL -

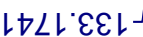

L68L'ท七レ-

L9ZLGヤL

IELO०OSᄂ -

0เt769レ

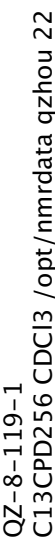
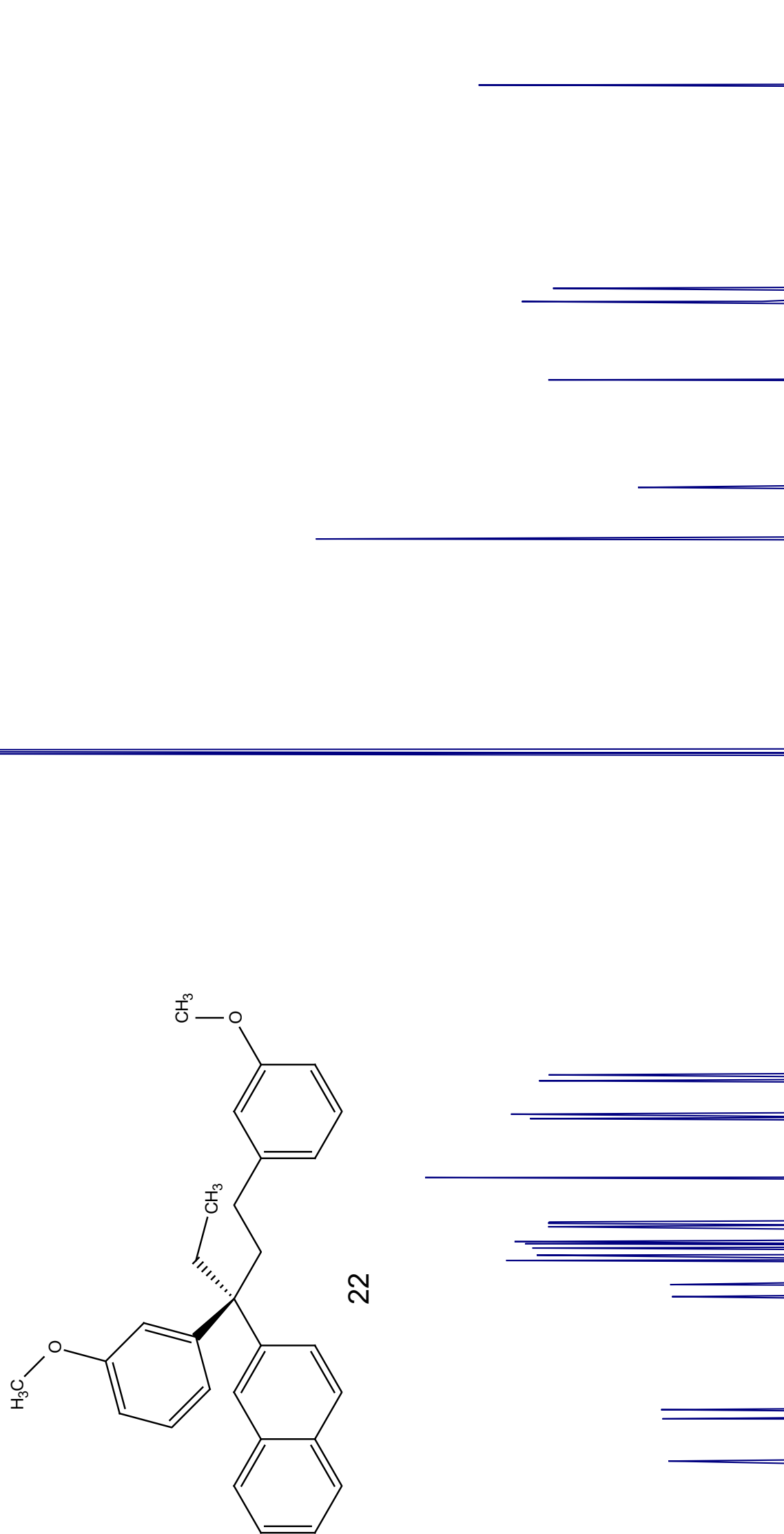

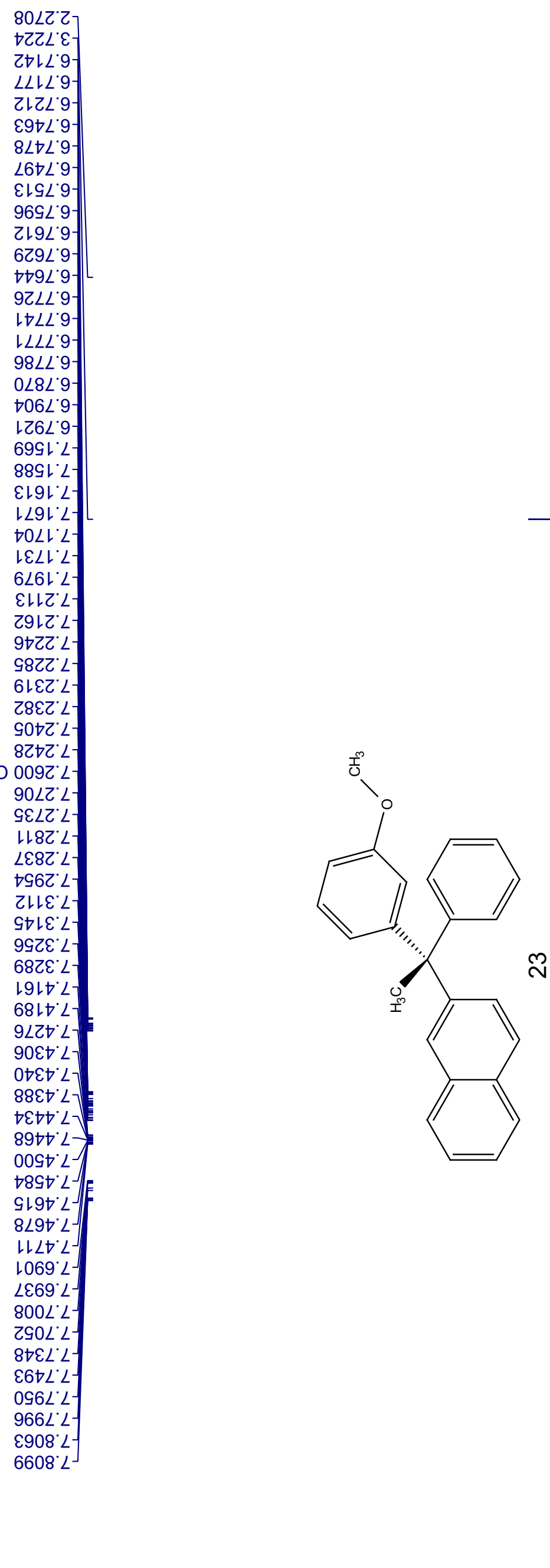

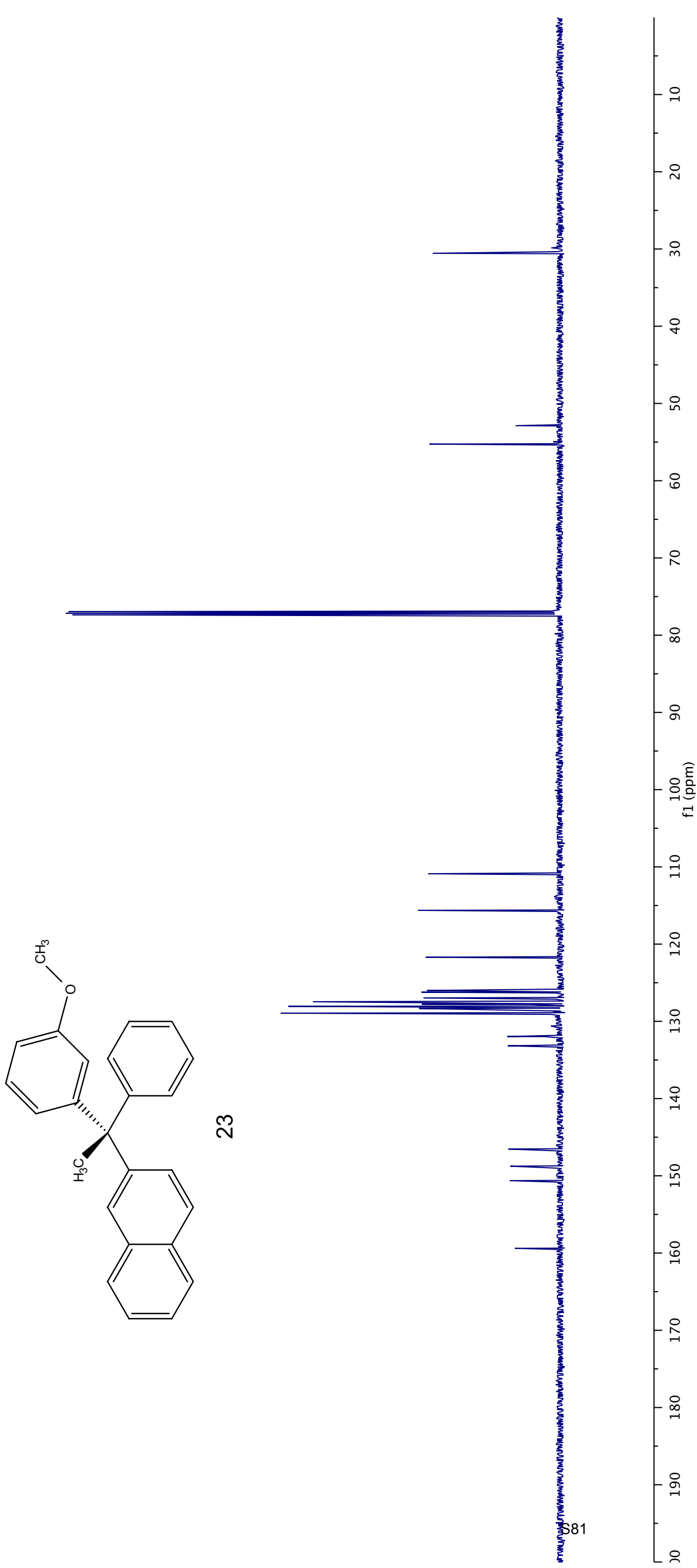
L608'0L LE89'GL GZ69เてレ] เ06レ9Zレ] †6ع9.9Zレ] [ GOEL LZL] $9 \nabla \angle Z \angle Z L] L$ GL70.8ZL 8L98.8ZL เ十98.8ZL 8L06 8ZL689Z6Z! †8\&8 $8 \varepsilon$ Z006.0七レ9ع6เ8tレ 9L66.8ㄴง6ฤ8.0งเ

วง98.69เ-
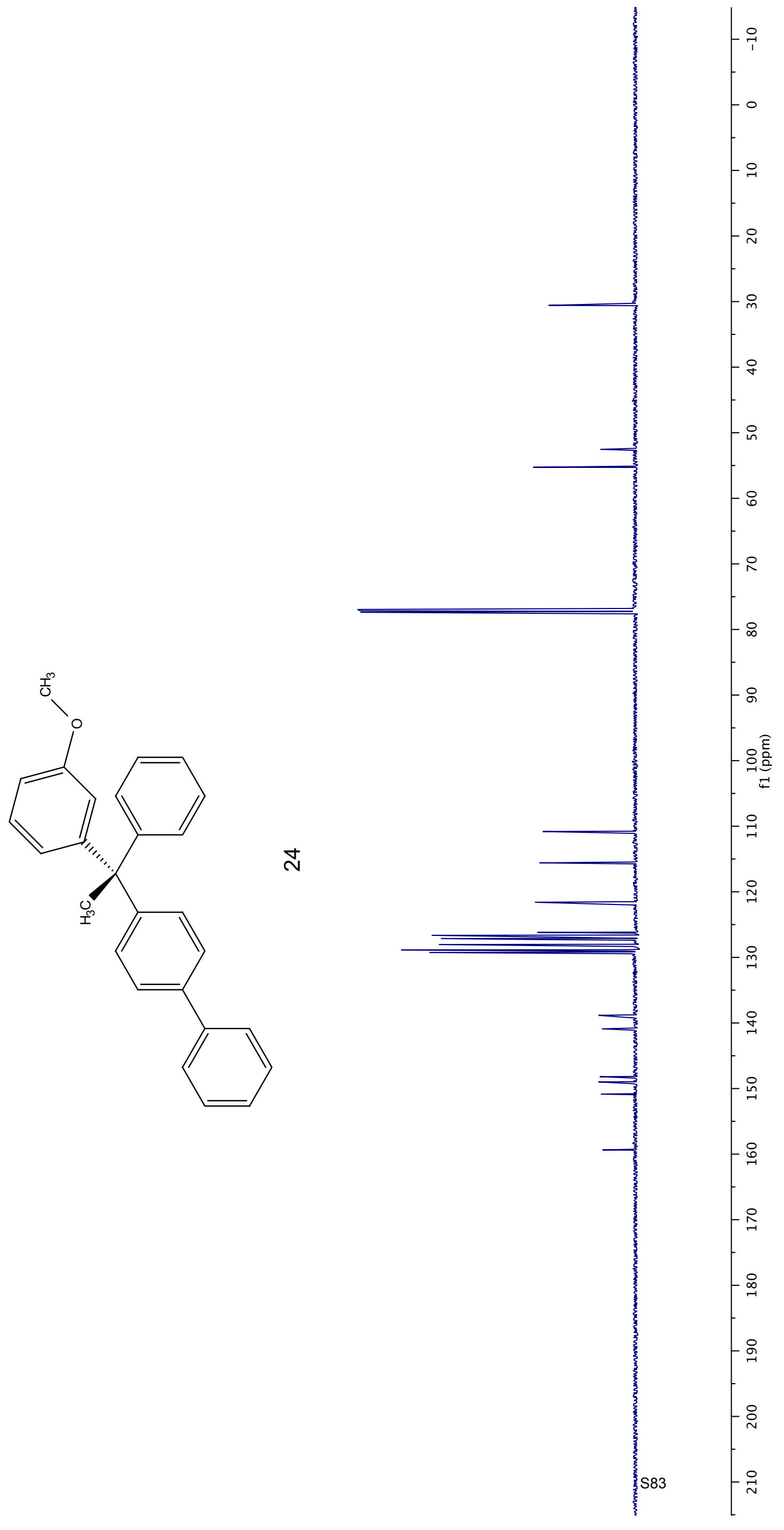
$1208^{\circ} 0$

S†L8:0

$6928^{\circ}$

9๑26.

乙Zルレ

8GL'Z]

88ル乙]

乙8乙レ乙

हLEL Z

†0เレ'乙-

9とヤレて

乙ESL乙

Z9Gl'
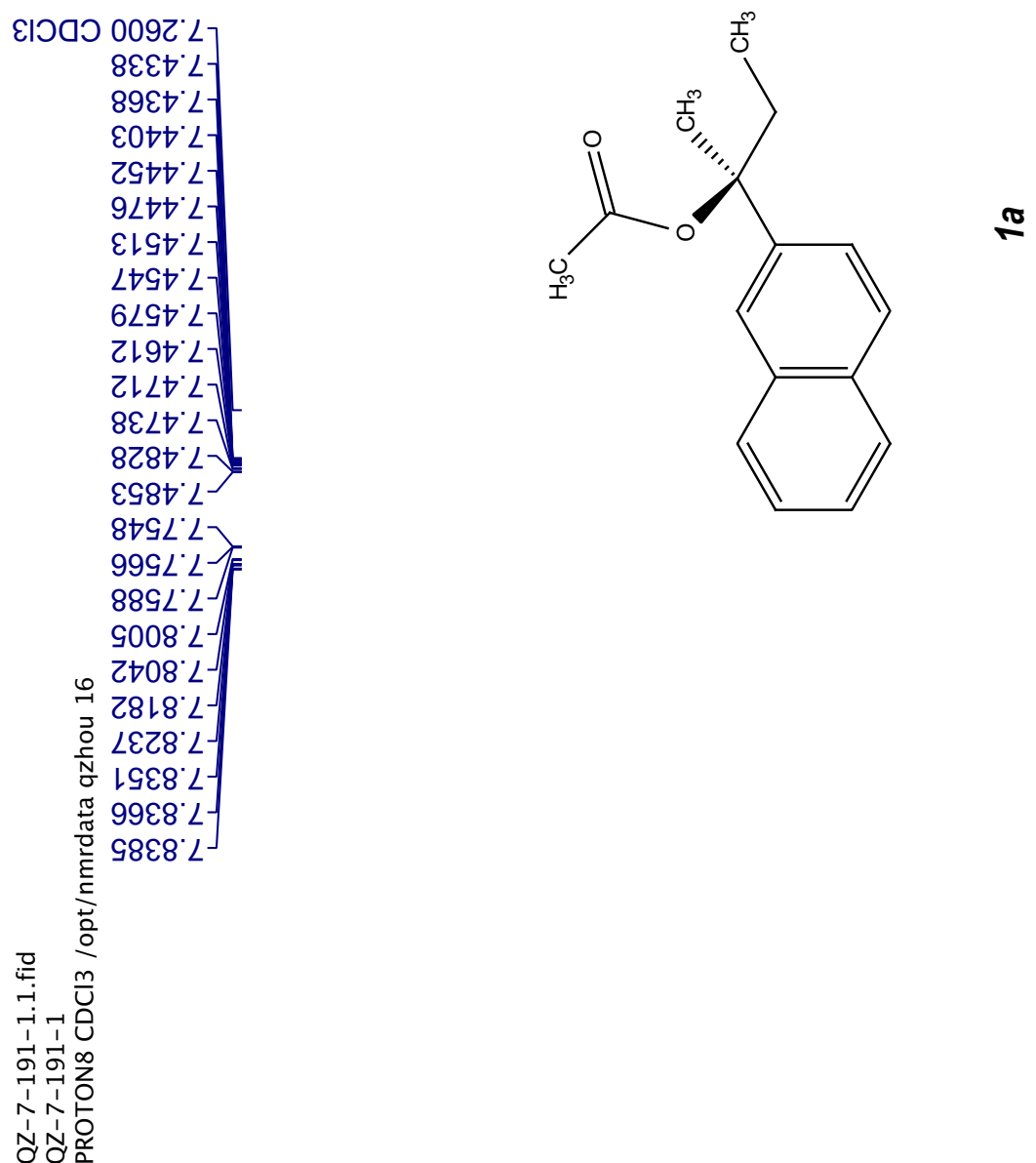

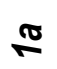

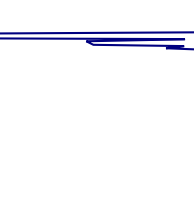

FTSO"દ o 
๑९乙๕'8-

86LE乙Z-

カZレG๋て-

ᄂ $60^{\circ} 9 \varepsilon-$
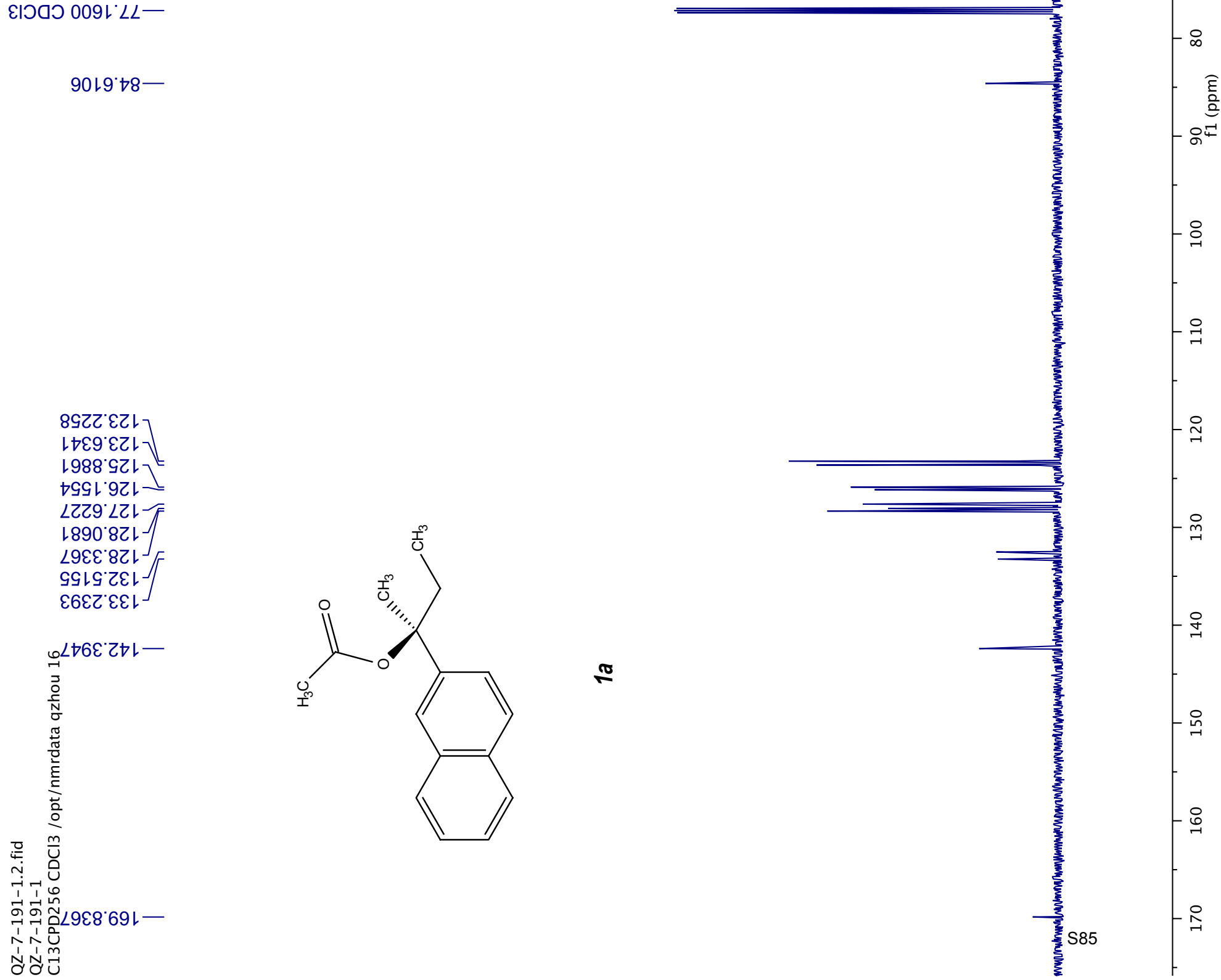
$188 L^{\circ} 0$

$7008^{\circ} 0$

$8218^{\circ}$

$6206^{\circ}$ เ

$\angle \varepsilon 80^{\circ} Z$

$0680^{\circ} Z$

$1960^{\circ} Z$

$\angle \supset 0 L ' Z]$

ててルてて

LLLZC

งฺてレて-

G6てL'乙

LLEL' $ح$

Lレナレ'て

0Zเ6'ย-

S90L'L

LOLLL

GLZL $L$

$\angle L E L \angle$

乙てヤレ:

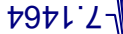

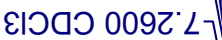

GS6E $\angle]$

$\left.886 \varepsilon^{\circ} L\right]$

$660 \nabla^{\circ} L$

IELTL

S9L9 $\angle$

$\angle 6 \angle 9^{\circ} \angle-$

$\angle Z 69^{\circ} \angle$

LOLLL

TOL L L

$\rightarrow G Z L L$

ㅁำ

吾

중

ह

\%

한 $\frac{m}{4}$

$1+1$
-1

ㄴํํㄴํํㅇํㅇ

1 1 क o

ㅊํㅇํํ음
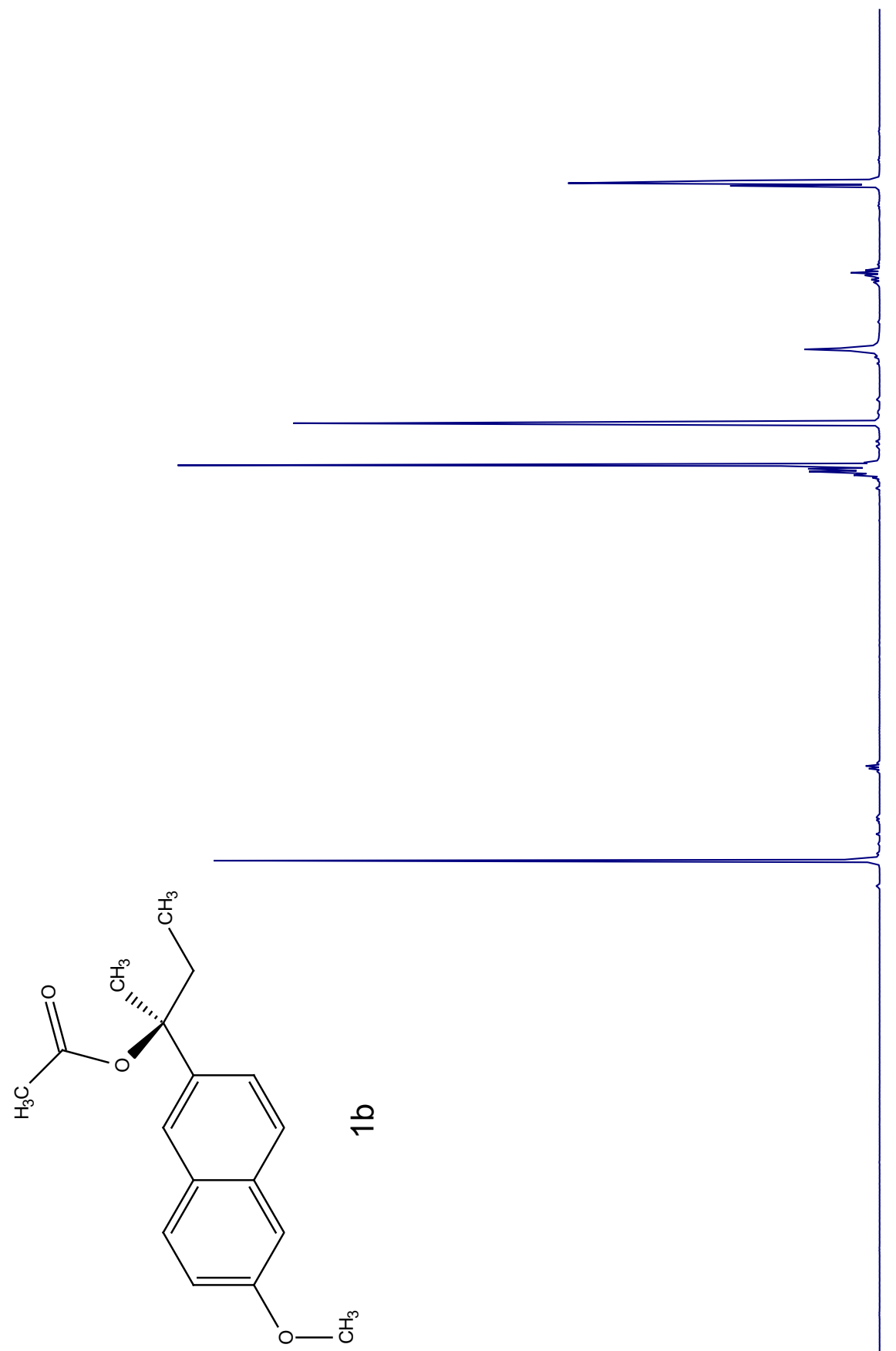

F8IT ह

I $280^{\circ} \varepsilon$

-62T. $\mathrm{s}$

$\stackrel{n}{\sim}$

$\circ$

in

I $8 \mathrm{SI}$

$\stackrel{+}{+}$

$\stackrel{\leftrightarrow}{\sim}$

हิ

(

in

$\circ$

in

6

$\stackrel{\circ}{\wedge}$

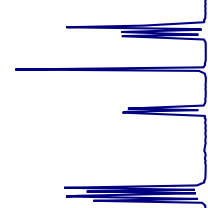

王6 $600^{\circ}$

工 000 "

두은

I $950^{\circ} \xi$

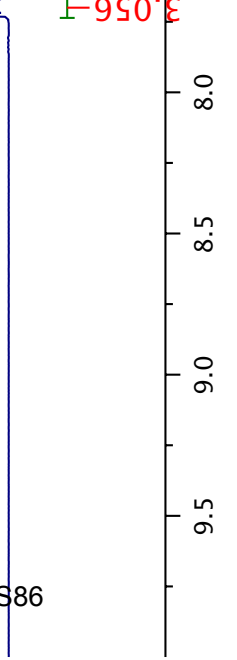


¿E98 0

$8998^{\circ} 0$

乙8L8 0

9レ乙0乙

$\angle 9 \varepsilon 0^{\circ} Z$

レLZZ

G6乙2 乙]

カレナ乙て

929ट⿱一

8†9Z乙

乙LLZ Z

09カ7て

৩LSt乙-

G69t $ح$

808t 乙

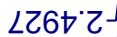

ZSOS'乙

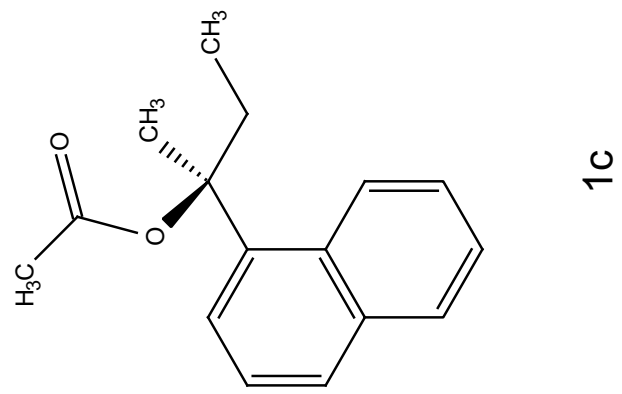




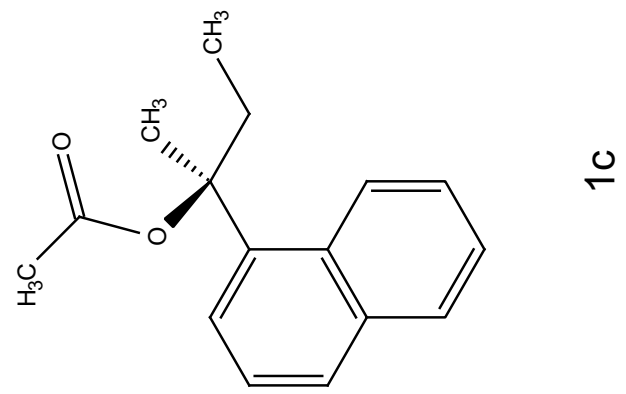




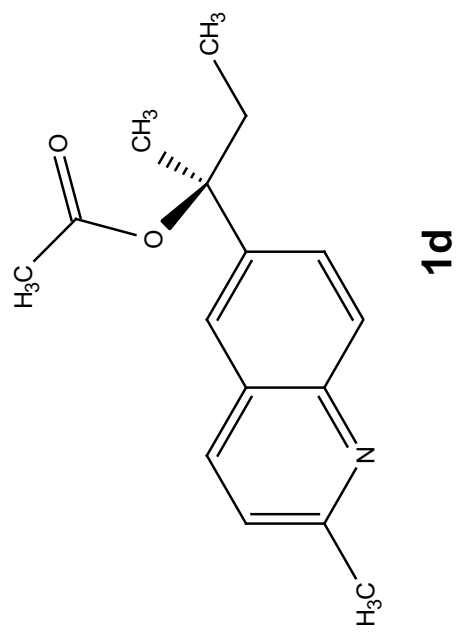




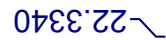

SLOt

90\&t G乙

レัOดะー

เ886.89レ-

E6L8.69!-
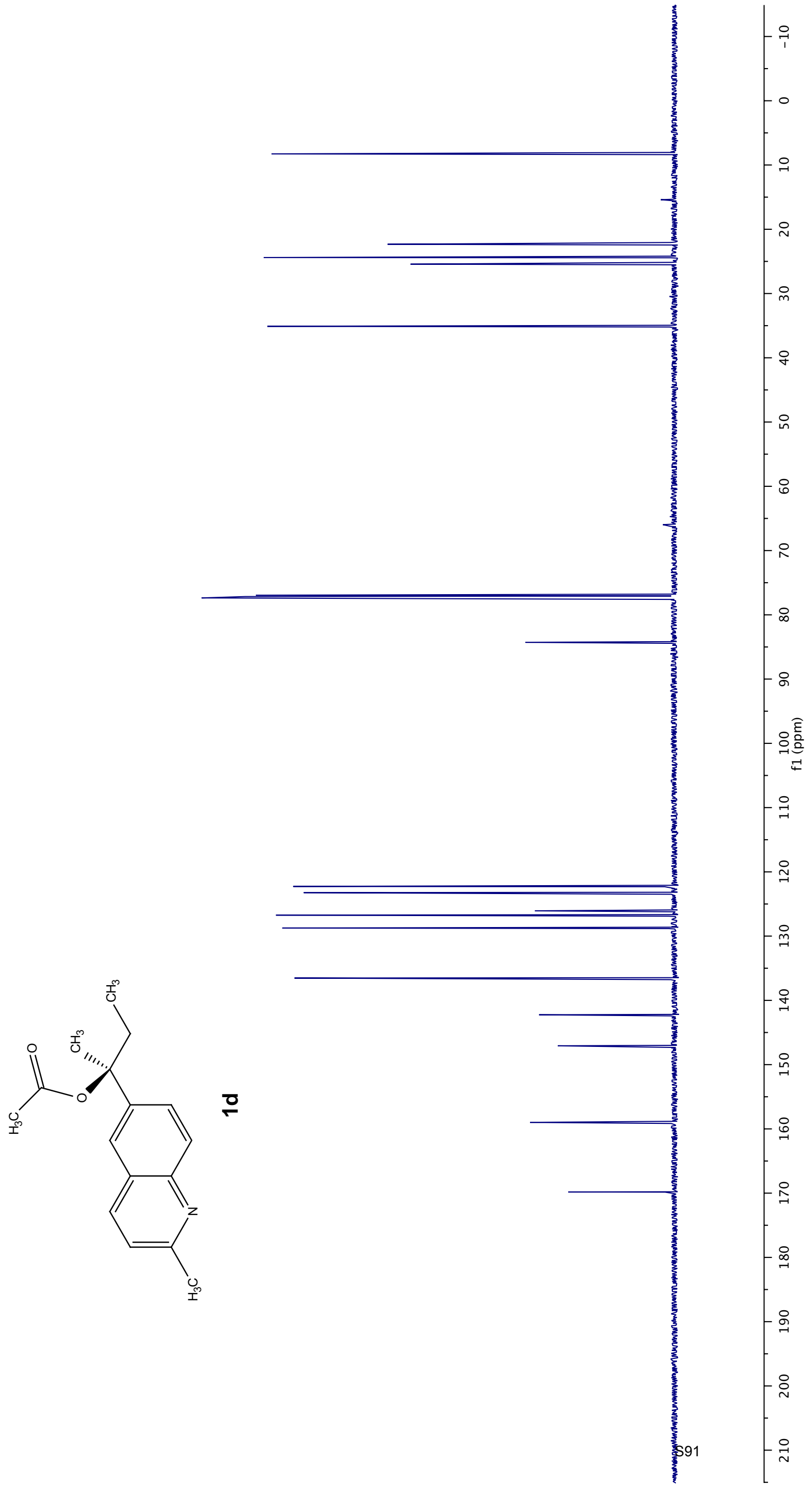


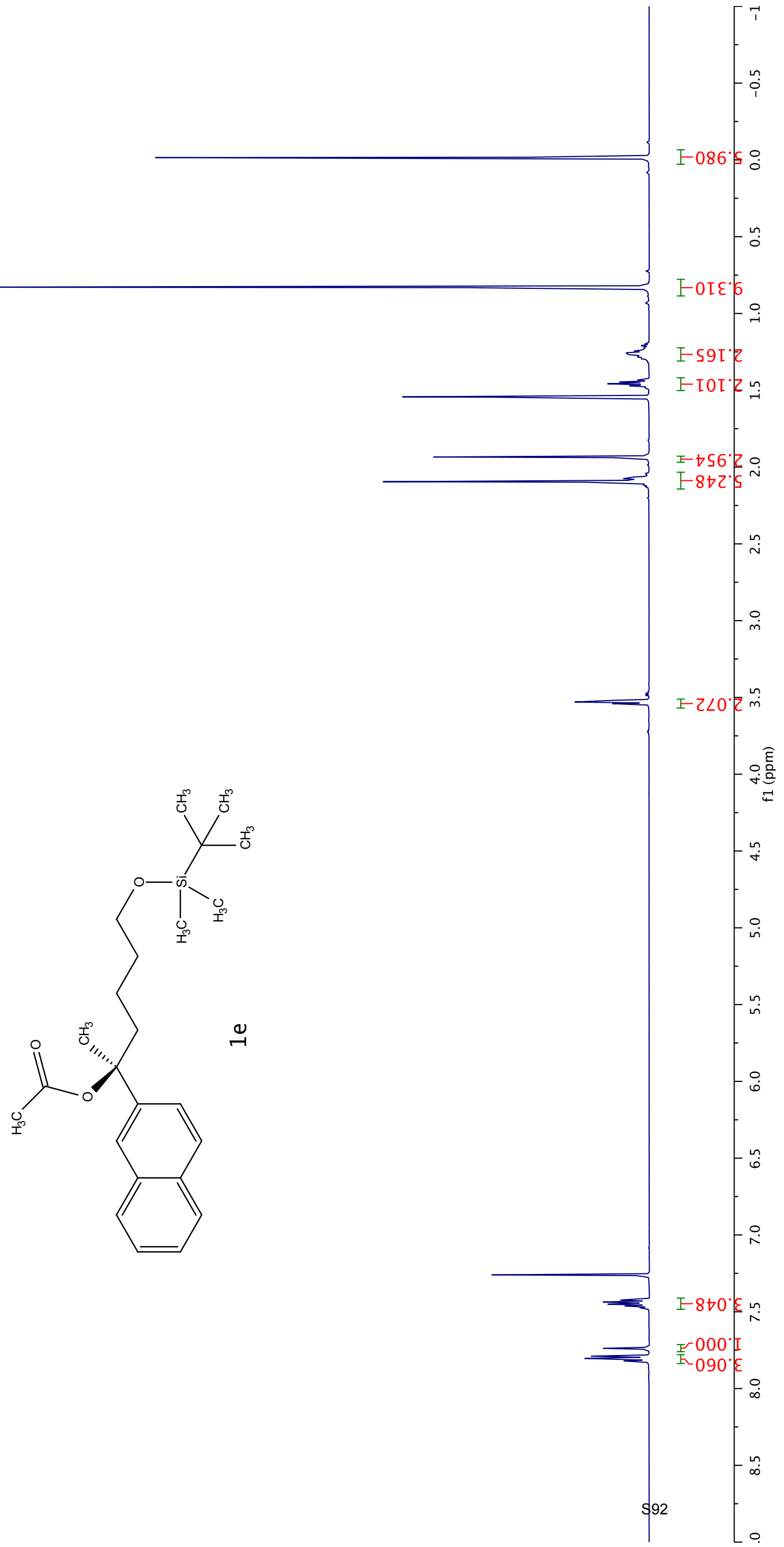


$6 \varepsilon 60^{\circ} \varepsilon 乙 レ$

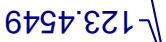
$\angle E \angle 8^{\circ} \mathrm{GZL}$ て8ヤレ・9Zレ_ EZ09 LZL $\bar{\Gamma}$ เ80L'8ZL9ZZع'8Zレ 0Z9†'ZEレ-

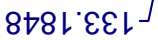
0乙て૬'てヤレ一

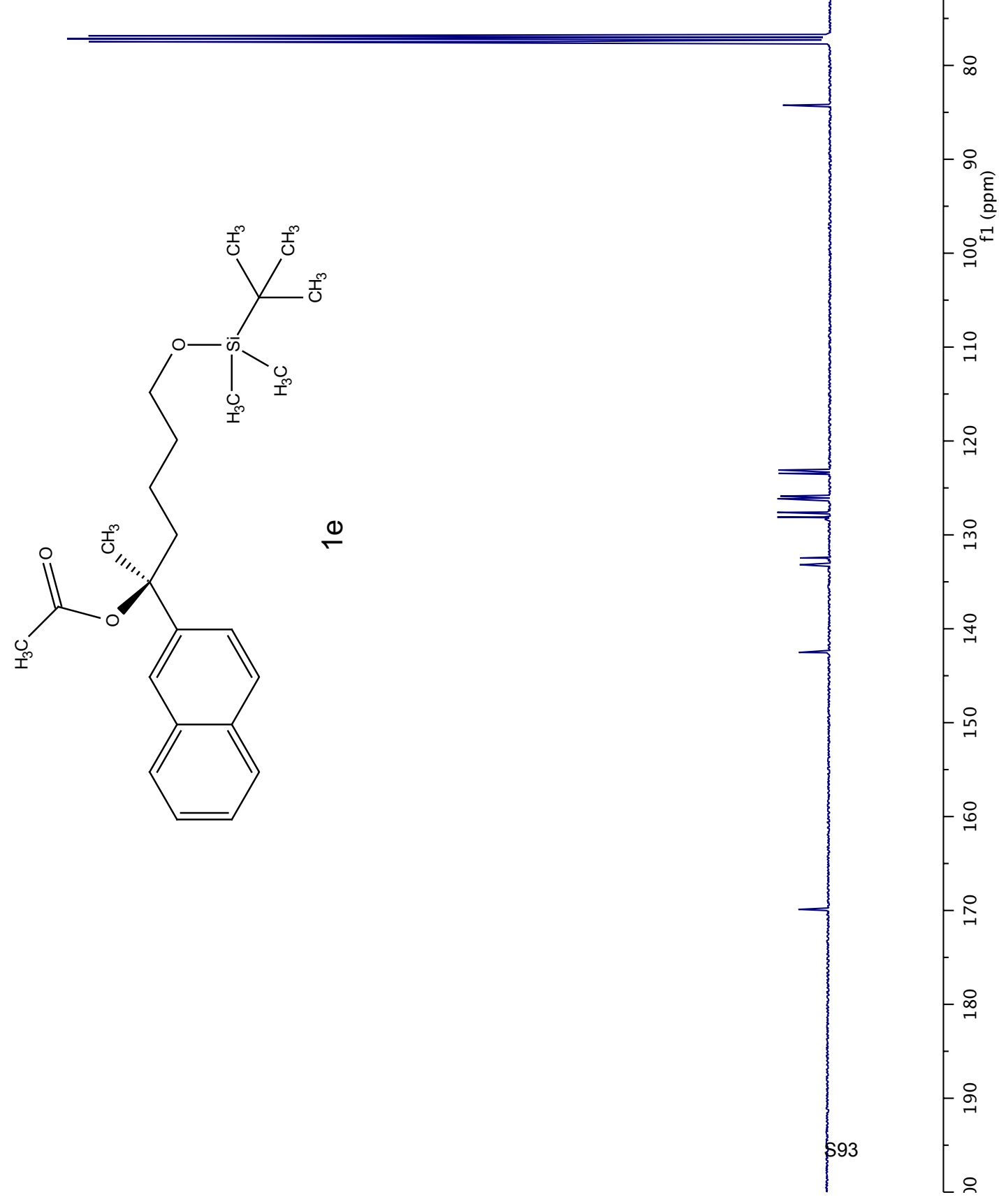




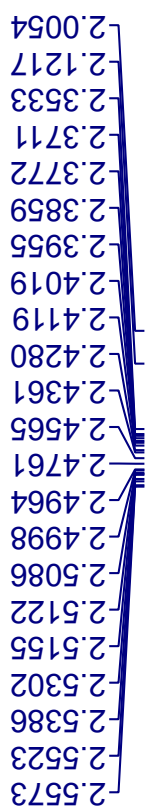

$6 \varepsilon 60^{\circ} \mathrm{L}$

$0 \angle 60 \angle$

$900 \mathrm{~L} L$

$980 \mathrm{~L} L$

8ELLL

LLIL $L-$

ZtGL:

GZLL L

EEZ乙 $L-$

ZLZZ L

L6EZ $L$

G乙大乙 $L$

$69 \nabla Z \angle-$

$009 Z^{\circ} L$

LZヤた L

ZLtV L

009t $L$

$\nabla t 9 t^{\circ} L$

$669 t^{\circ} L$

$19 \angle t^{\circ} L$

$\angle E 8 \nabla^{\circ} \angle$

$6 \angle 8 \nabla^{\circ} L$

GZ6t $\angle$

$S \angle 6 b^{\circ} \angle 7$

SGOG $L$

OOLS L

$2808^{\circ} \mathrm{L}$

†EL $L-$

$\angle I E 8^{\circ} \angle-$

S9ع8 $L$

L乙G

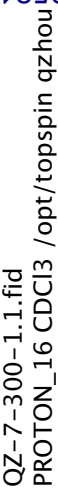
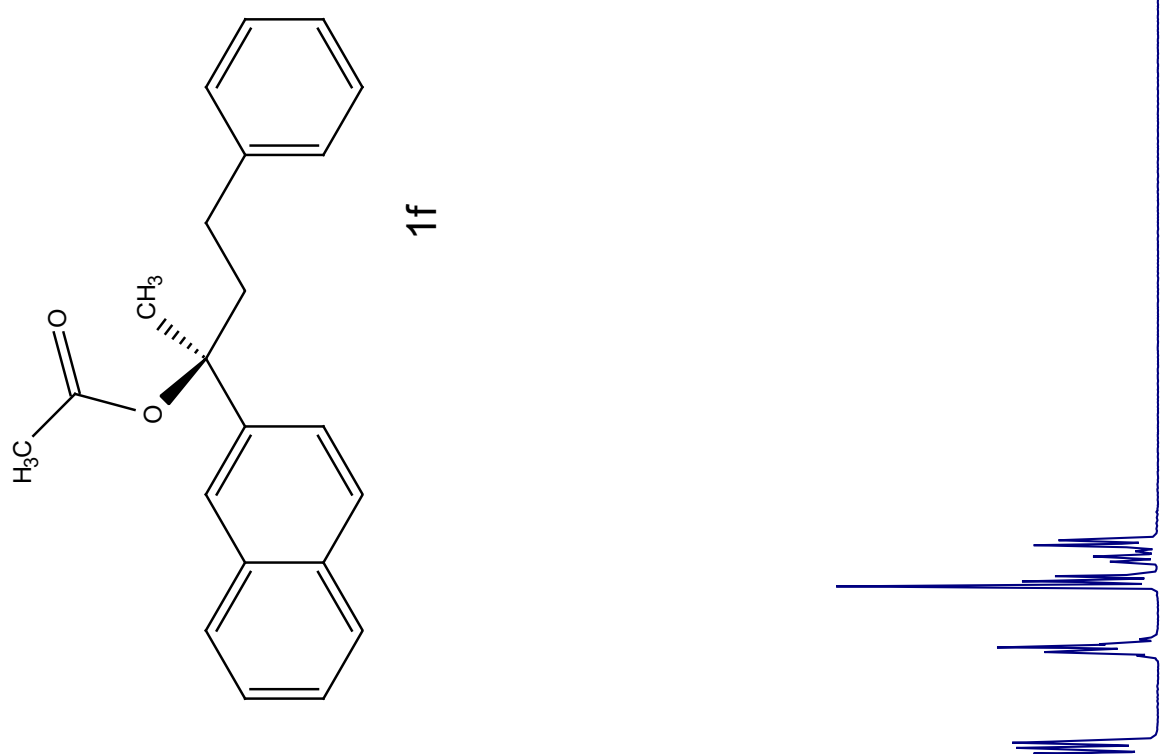

岳 0006 "

[802"

R $100^{\circ} \varepsilon \stackrel{\text { ? }}{\sim}$

F9t0.

$\stackrel{\infty}{\infty}$ 
9カてォててー

9レย'G乙-

ட6เจ゚0E-

86L0 ヤレー

เ૦ย0"จ8-

เ9Z0๕๕乙レ

ह8LG'EZL

乙896'G乙L

\&Gเ0.9ZL]

†ELZ'9ZL]

G $\triangle 9^{\circ} \angle Z L$

†88乙 8Zレ

6Z9E 8Zレ-

6Z97 8Zレ|F

906t 8ZL

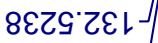

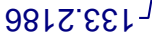

†とヤ8レレレ

ட90Zてヤレ
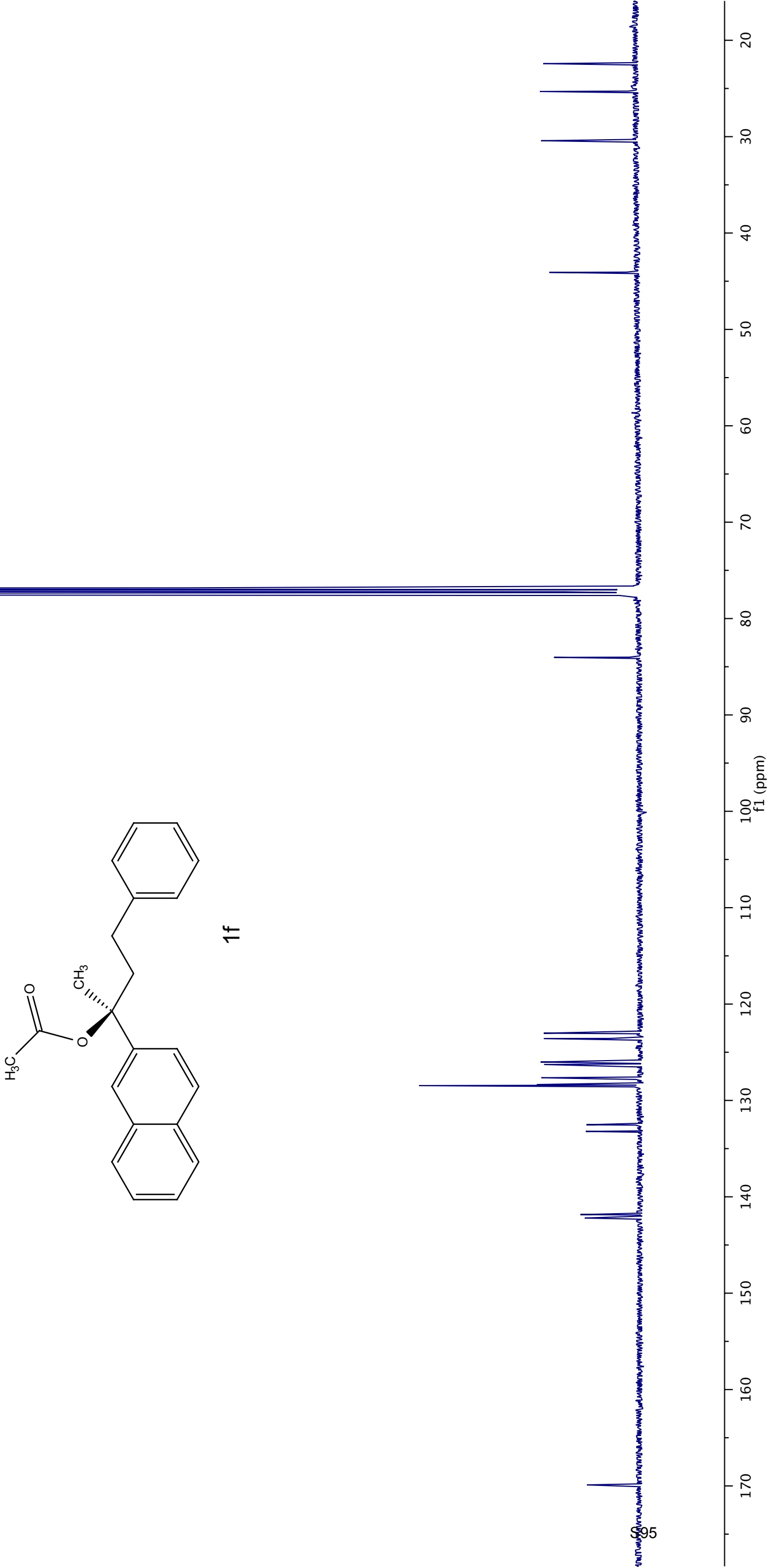
06เ8ㄴ -

0レてヤててー

ZLGZGZ-

ง99レ๋นレ

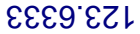

$\angle 9 \angle 8^{\circ}$ GZL

GSZ0.9ZL

$\varepsilon \angle \nabla 0$ 9ZL

G6ZL'9ZL

Z09乙'9ZL

เ8ZE' LZL

99t9 $\angle Z L$

GZZZ 8ZL

086ध'8ZL

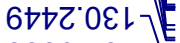

8890 ZहL]

G609'Zع -

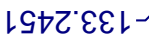

LOGZ GEL -

6†เ8.9ع -

เ96レてヤレー
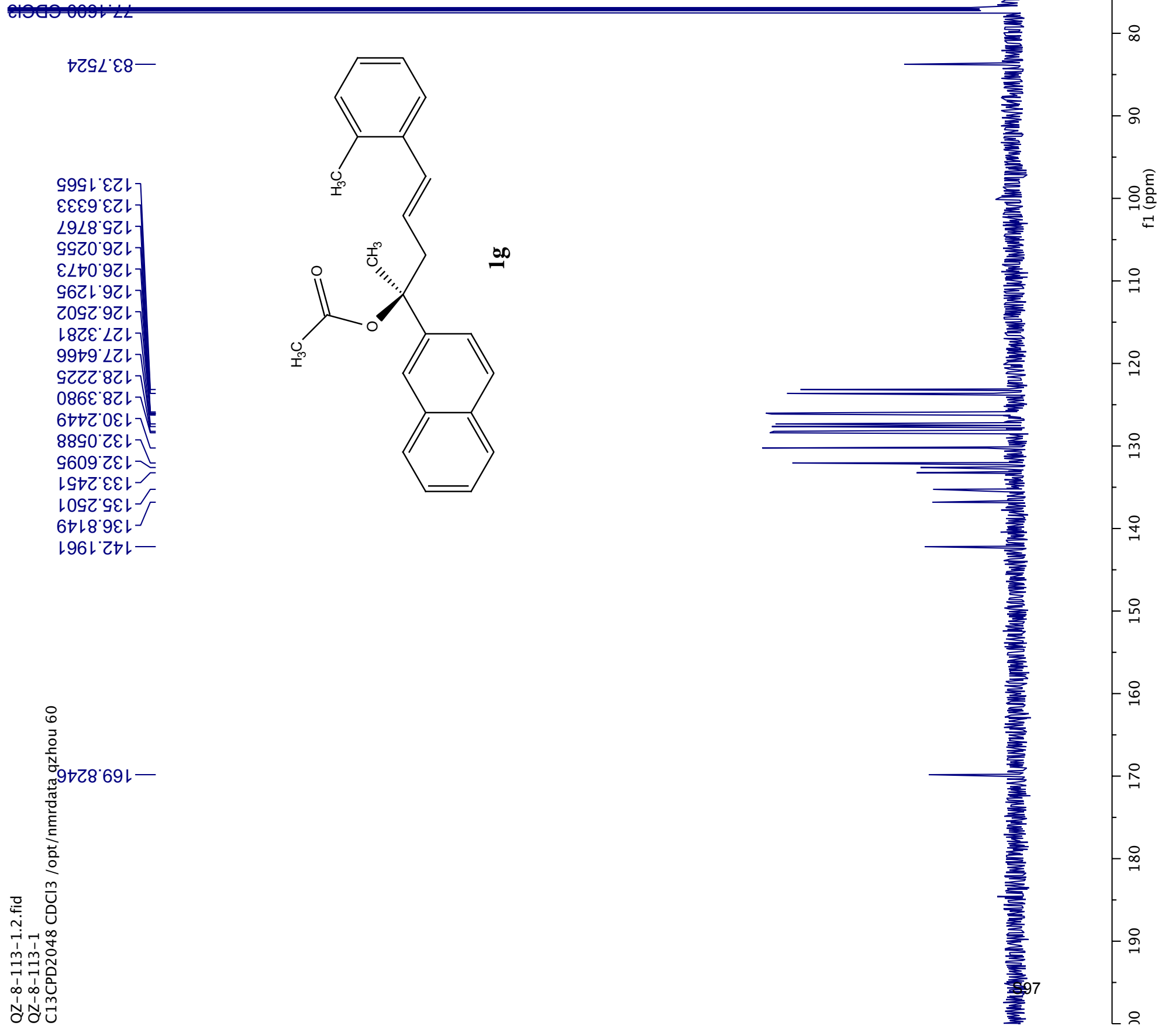


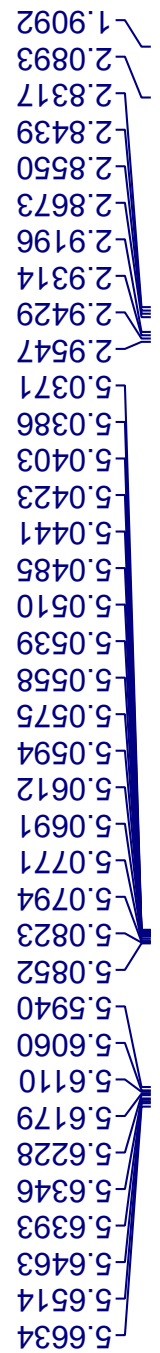

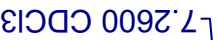

$\checkmark G E \nabla^{\circ} L$

8LET L

69t大 $L]$

†七七七 L

LESt $\angle \sqrt{ }$

ZLST $\angle V$

06St $L-$

0Z9t L-

089t L

乙E $\angle \nabla^{\circ} \angle$

ZZ8t L-

$\stackrel{m}{\rightarrow}$

$\angle \succ 8 \nabla^{\circ} \angle$

09GL'L-

6LSL $L-$

$009 L ' L$

$\checkmark 86 L^{\circ} L$

EL08 $L$

$\checkmark 808^{\circ} \mathrm{L}$

GEL $L$

6818' L-

हZZ8 L

$\angle 0 \varepsilon 8^{\circ} L$

¿ZE8 $\angle-$

†๑\&8 L

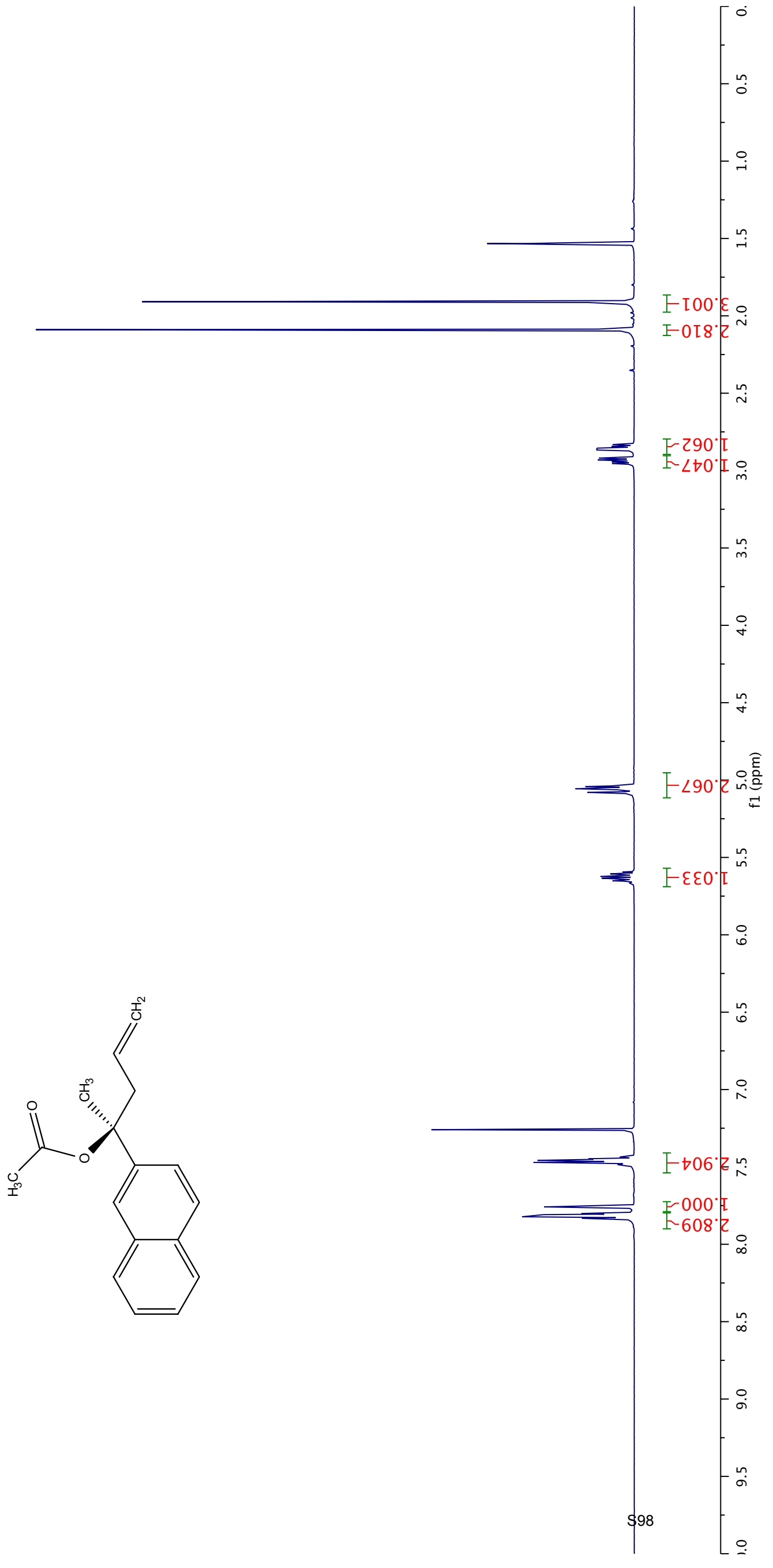


0†9६てて—

LEOLGZ-

920t $97-$

889ع'ع8-

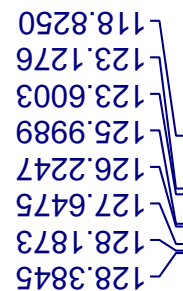

ᄂL89'ZEL-T 0Z96'ZहL-

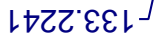

096レてヤレー
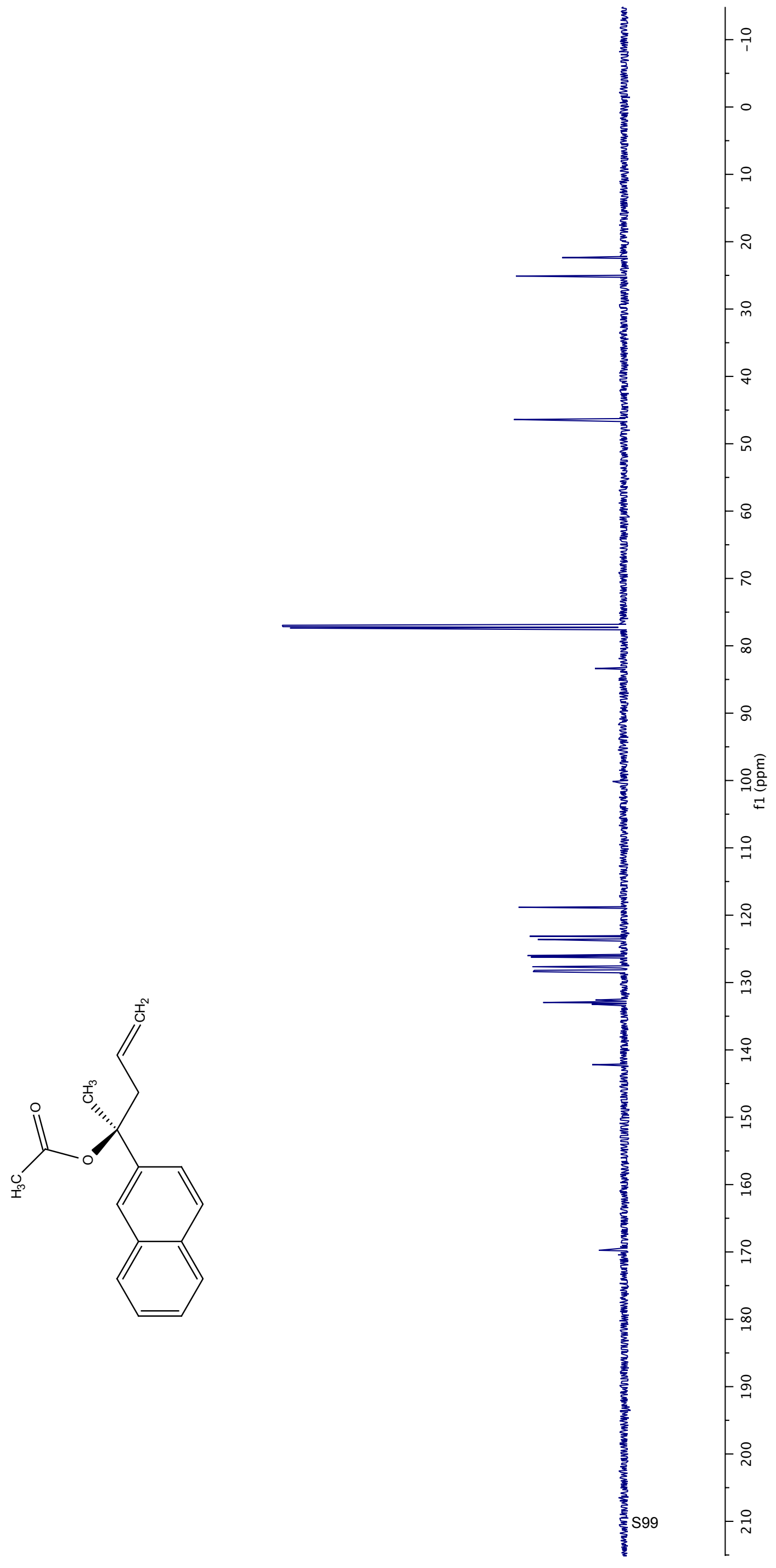

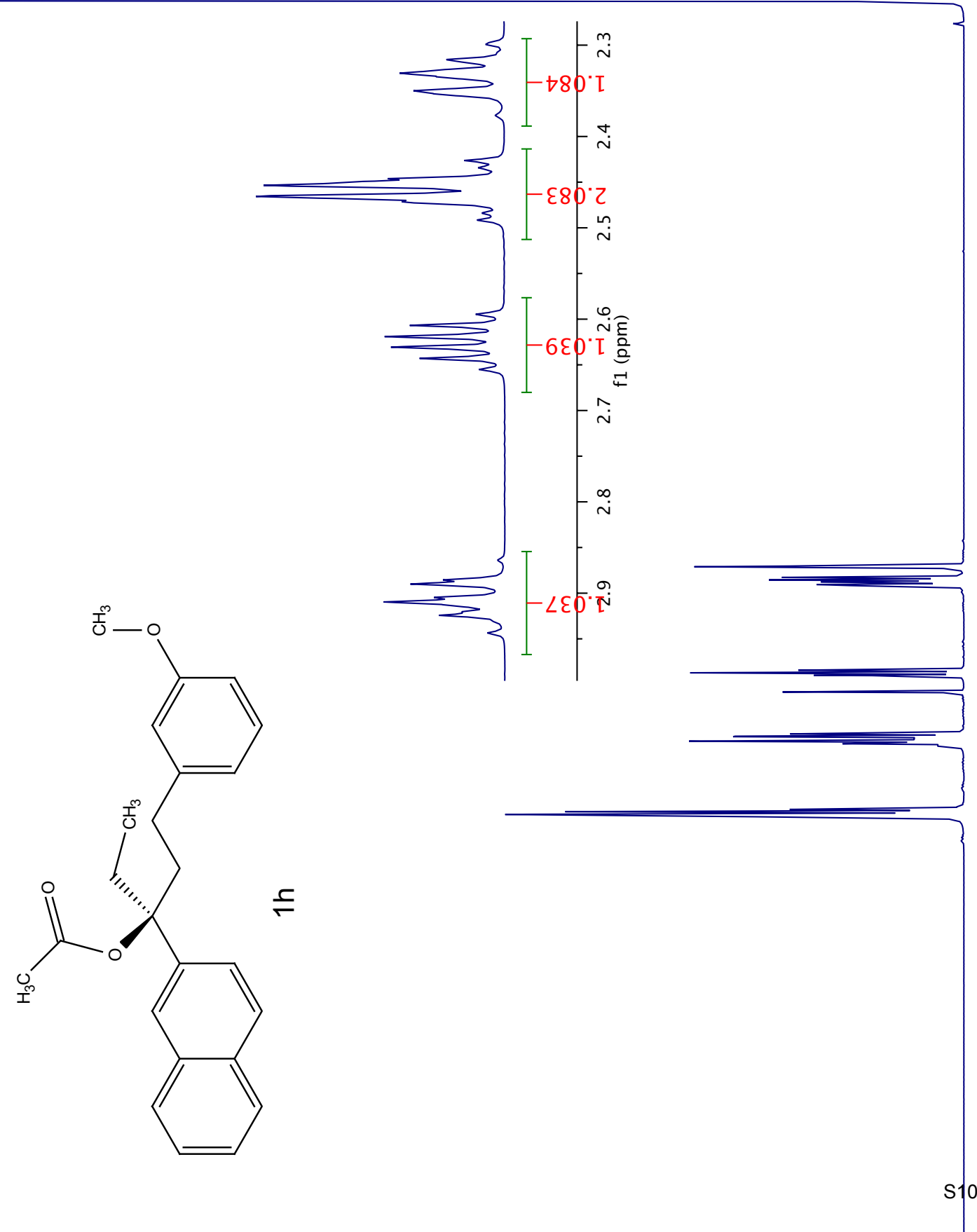
乙৪レててー

ZZZレ'0\&

89Z6.0

†90七 $6 \varepsilon-$

68จZ'SS-

ยІวดว 009L'LL-

St9L'L8-

9ع0عレレー

98レナレー

$\angle 6 \angle 8^{\circ} 0 Z$ L

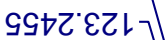

เ8ES $\bullet 乙 レ]$

GZL0.9ZL

レGZ9Zレ-

9LZ9 LZL-

0เ6L8ZL-

เELE'8ZL

8ยเナ゙ 6てレ

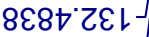

$\nabla L \angle L \varepsilon \varepsilon L$

GSเ9ำเ

6969'Eヤレ

8†ZL69ルー

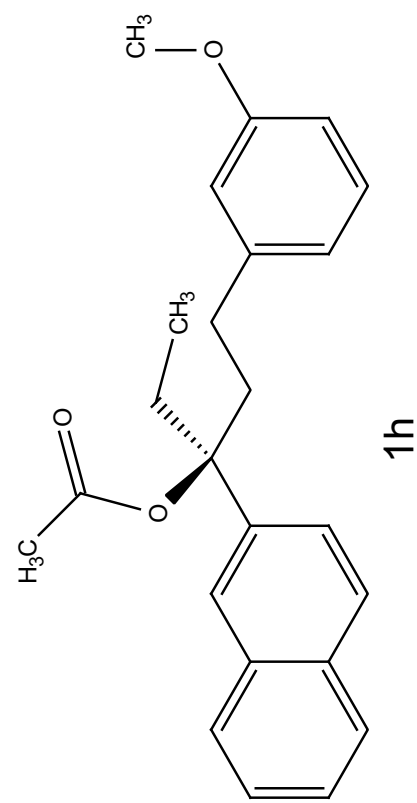


<smiles></smiles> 

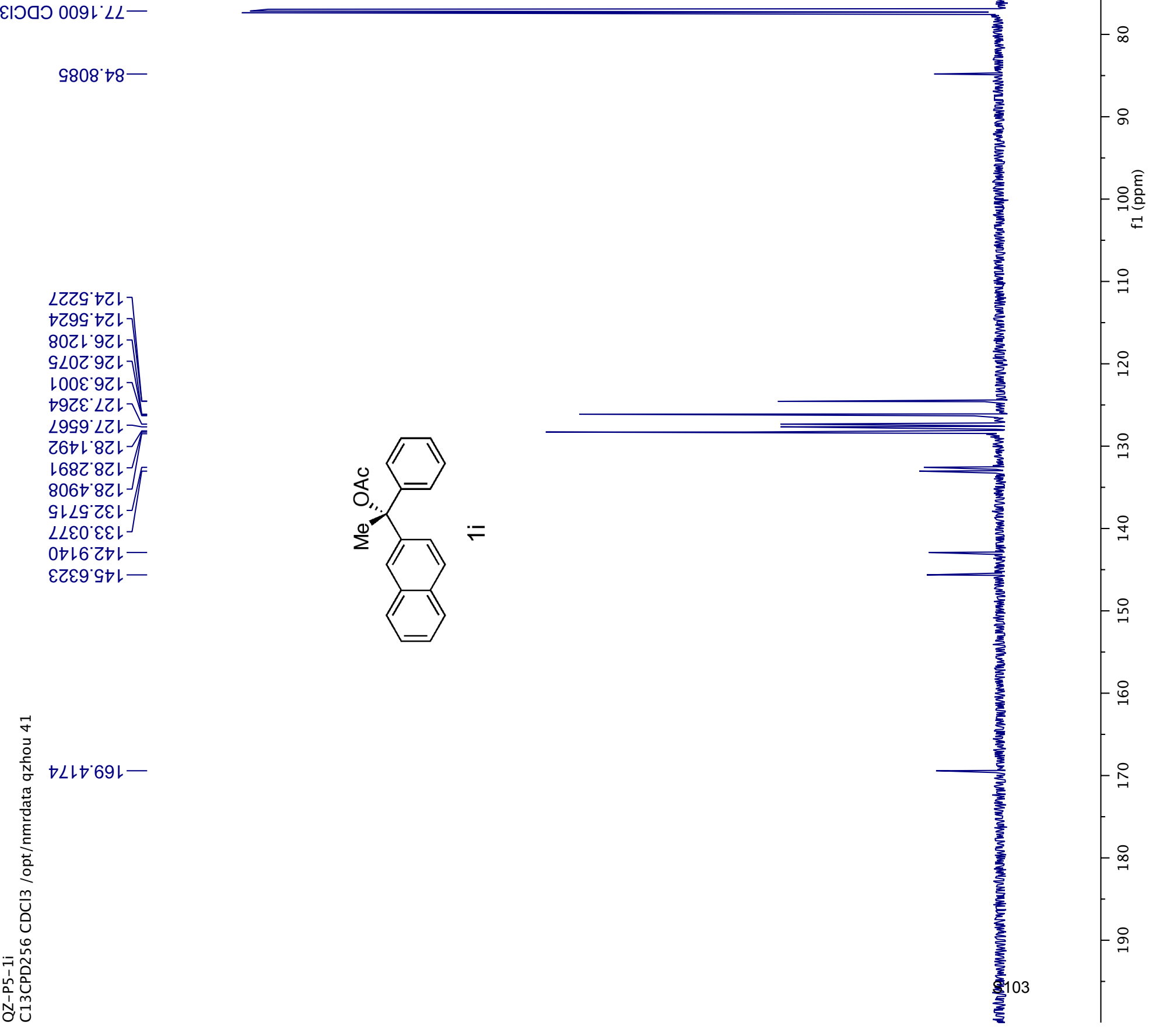


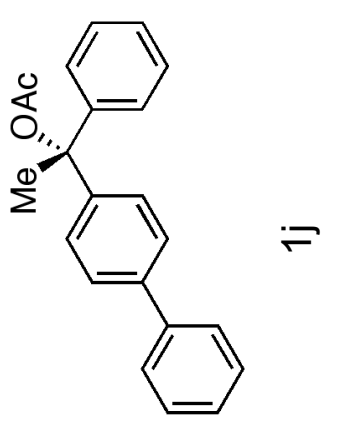




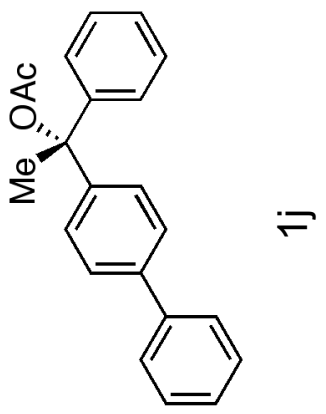




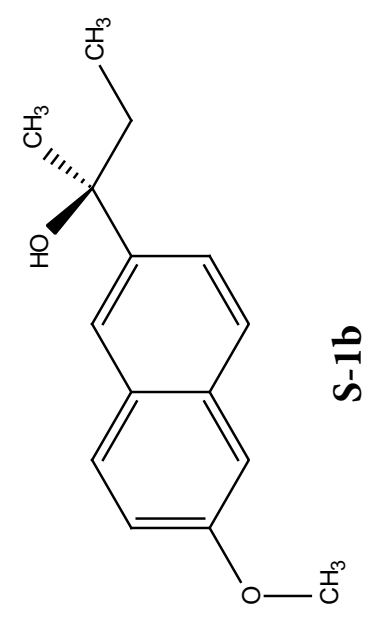

ต 
6LLLGL-

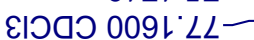

ELG9.9OL-

1626'8L 888Z'ยてレ

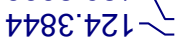

89Z8.9ZL 8L9L8ZL6ZEL'6ZL-

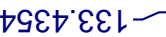

レE66てヤレー

9ZZL'LGL-

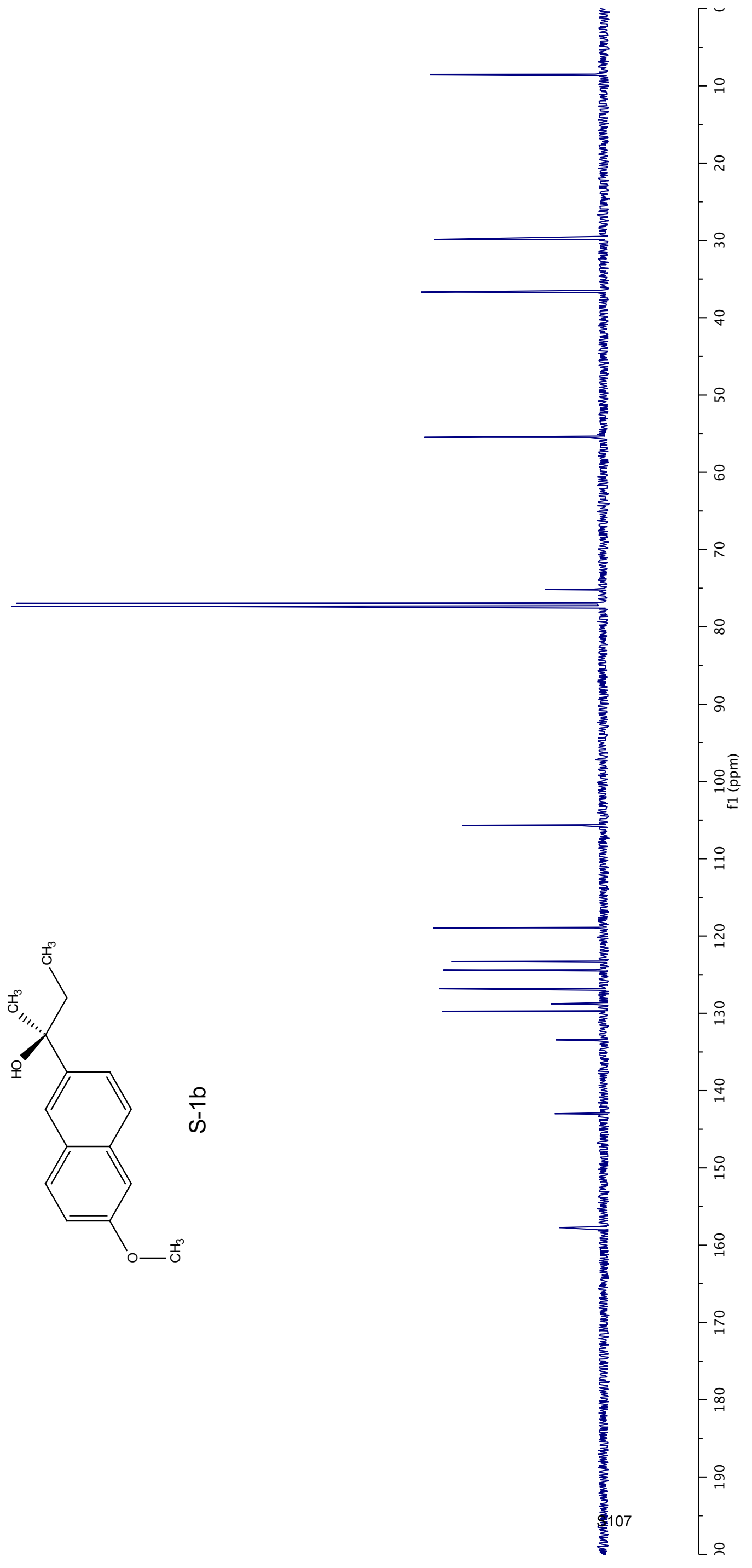


t大 180

$6928^{\circ}$

ع6ع8.

6ย乙8 เ

9L10 Z

七七8'乙

896l'Z

E80Z Z

レ0Zてて

$\left.\angle \nabla Z Z^{\prime} Z\right]$



乙LEZて

09七乙 $冗$

96カ乙 乙-

†เ9Z

$0 \varepsilon \angle Z^{\prime} 乙$

$\triangle 8 Z$ Z
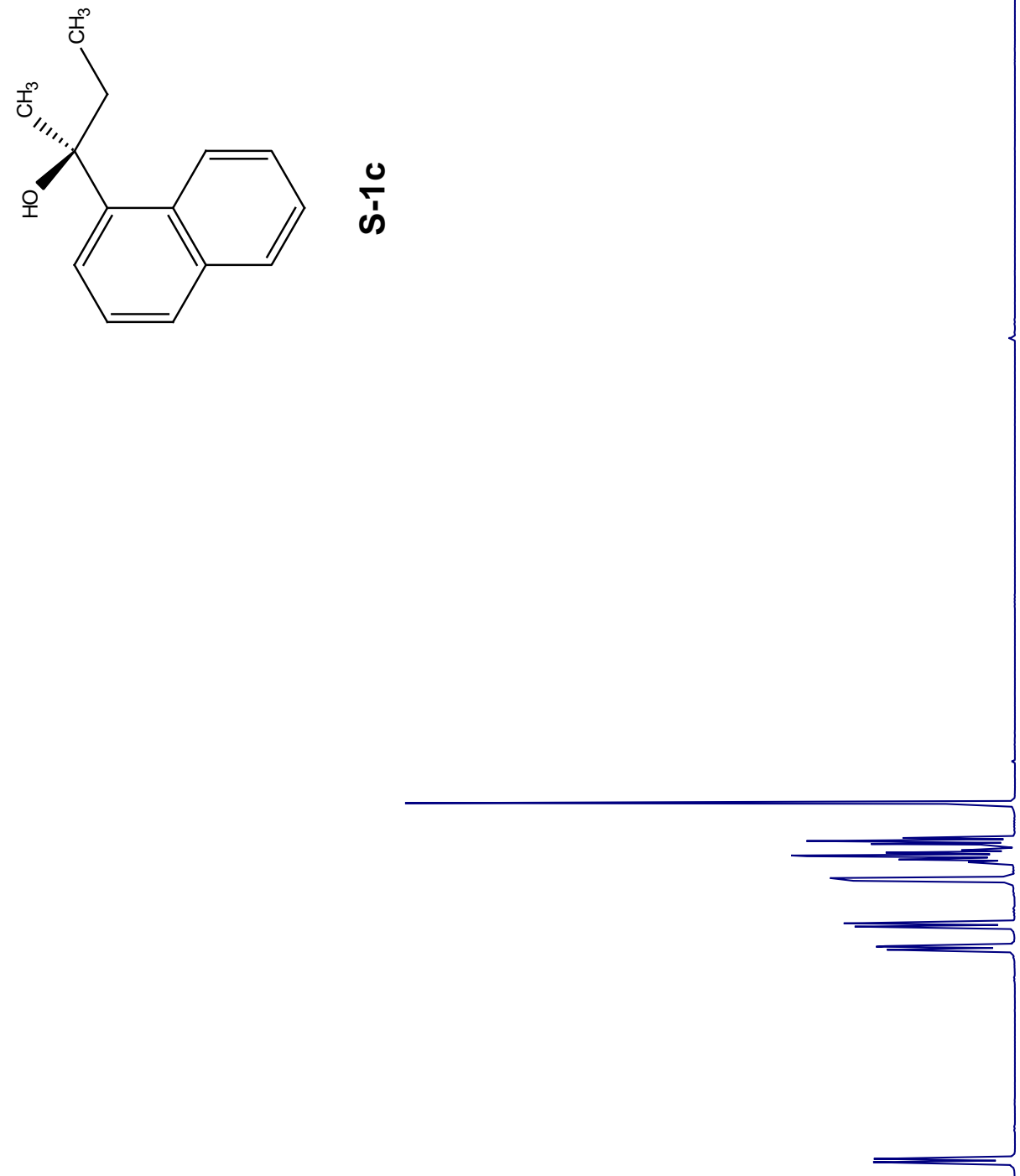

812.8 
†6เG'6Z-

06ฑฤ $9 \varepsilon$

GL88.9L

हाวดว 009! LL

08L0॰てL

\&698 †乙レ ELLて・GZL

6ยเย'G乙レ

$0880^{\circ} \mathrm{LZL} /$ F

0L69.8ZL-

0†9Z6Zᄂ

9960. $L\llcorner$

LZZ० GEL

ヤ9てヤ゙マー

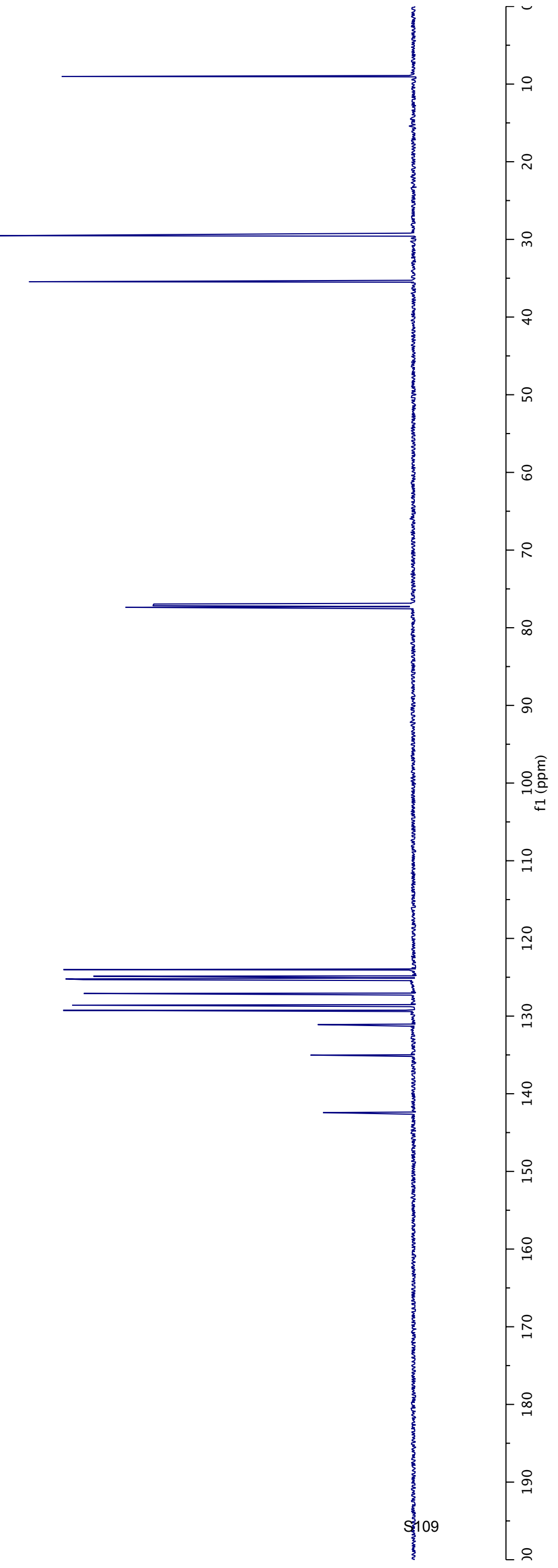


ZL6L'O

$9608^{\circ} 0$

$0 Z 28^{\circ}$

Eot9

OSL8 L

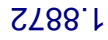

$6868^{\circ} \mathrm{L}$

$8016^{\circ} \mathrm{L}$

ОЕट6 $\mathrm{L}$

GZE6 $\mathrm{L}$

SGE6 L

$9+76$ เ

6996.

$9896^{\circ} \mathrm{L}$

9086' ᄂ

ZE66 ' -

8LEL' $冗$

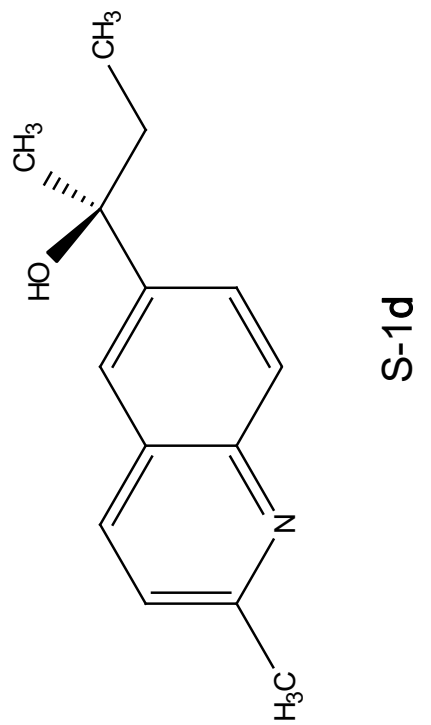

ì 
89Lt'GZ-

$\varepsilon \subseteq 00^{\circ} 0 \varepsilon-$

SLLL' $9 \varepsilon-$

乙ZGてててレ

0Lจレ๕とL

8ZLL9ZL

80レ゙டてレ

†レOS'9عᄂ-

9860'Gヤレ -

乙レナ0 LtL

ยเ68.8งเ-

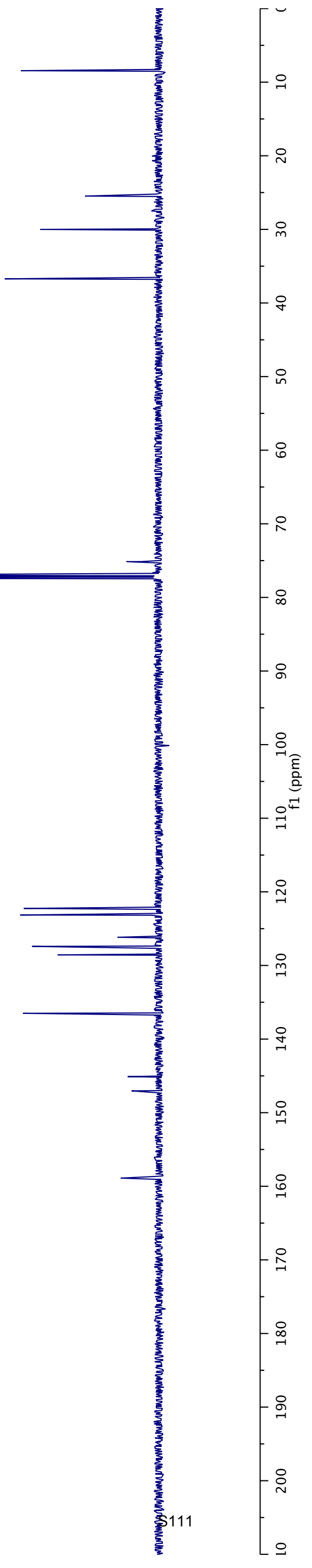

A

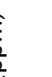




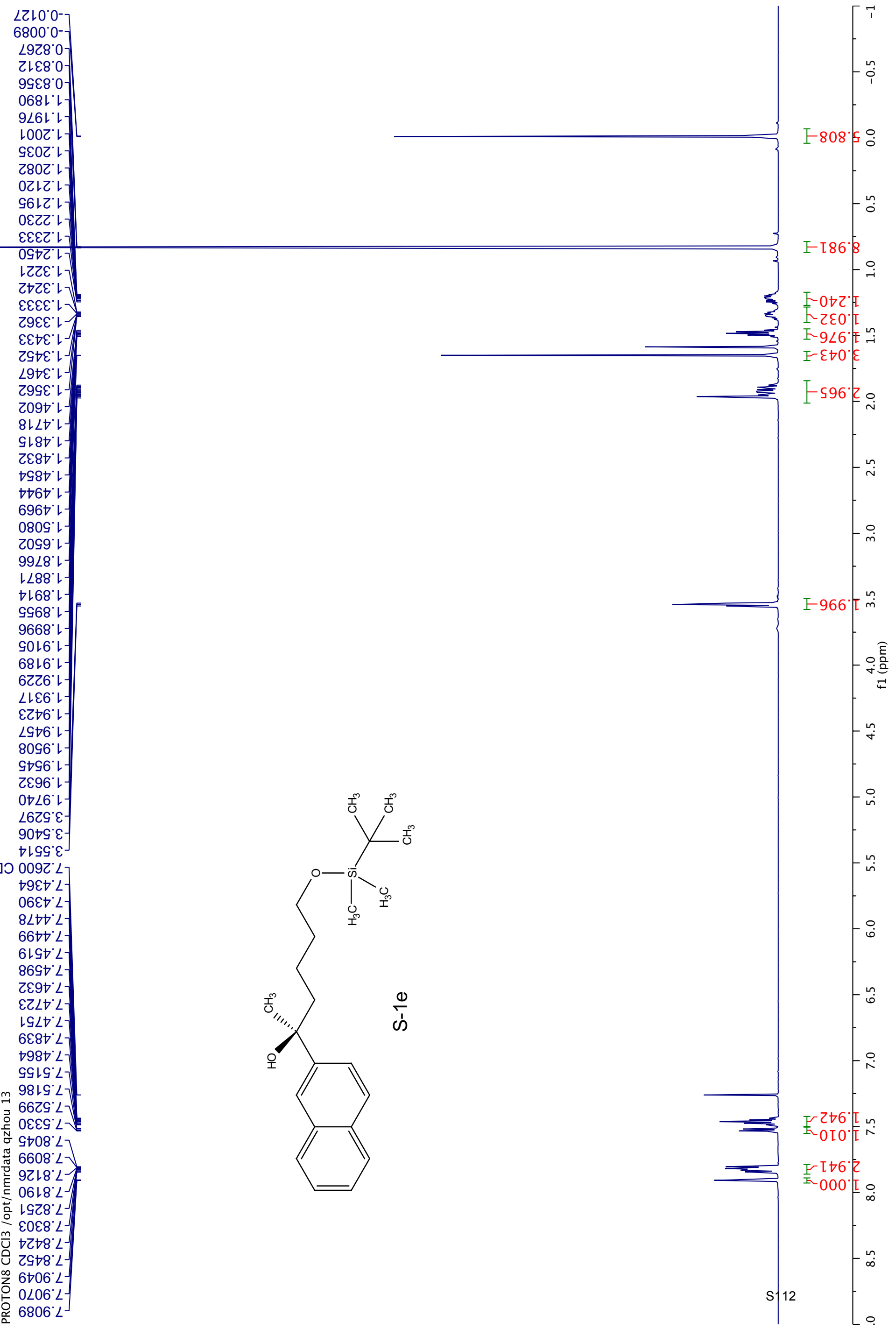


6L6で๕てレ 80Z8'と乙レ 9IGLGZL 66レ19ZL mG989' LZL$-8 \angle 96^{\circ} \angle Z L-$ 产レLZ゙8Zレ

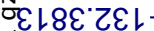

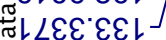

㟧山

鱼兴 。

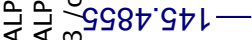

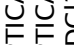

ㅇํㅇ

00
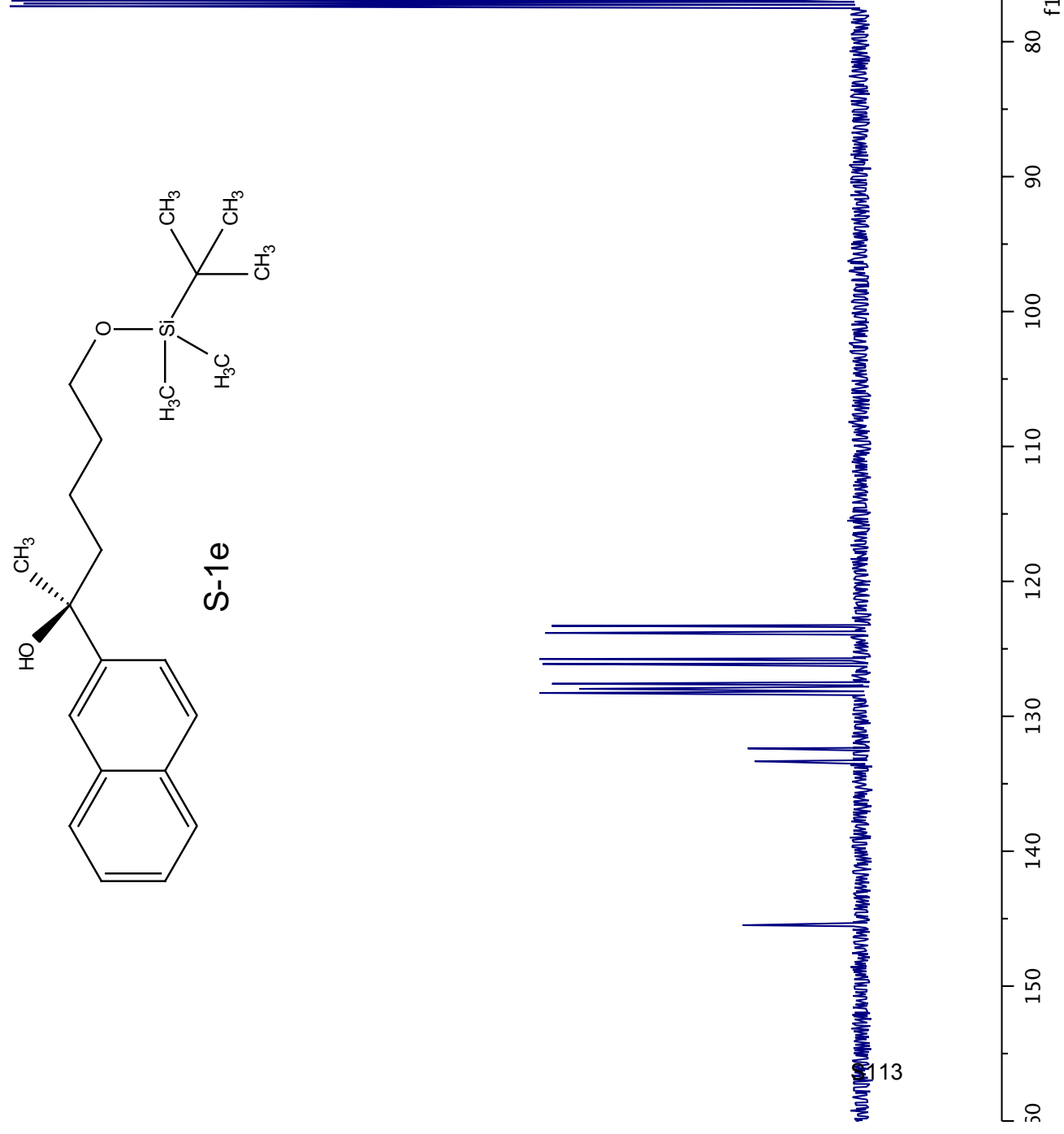


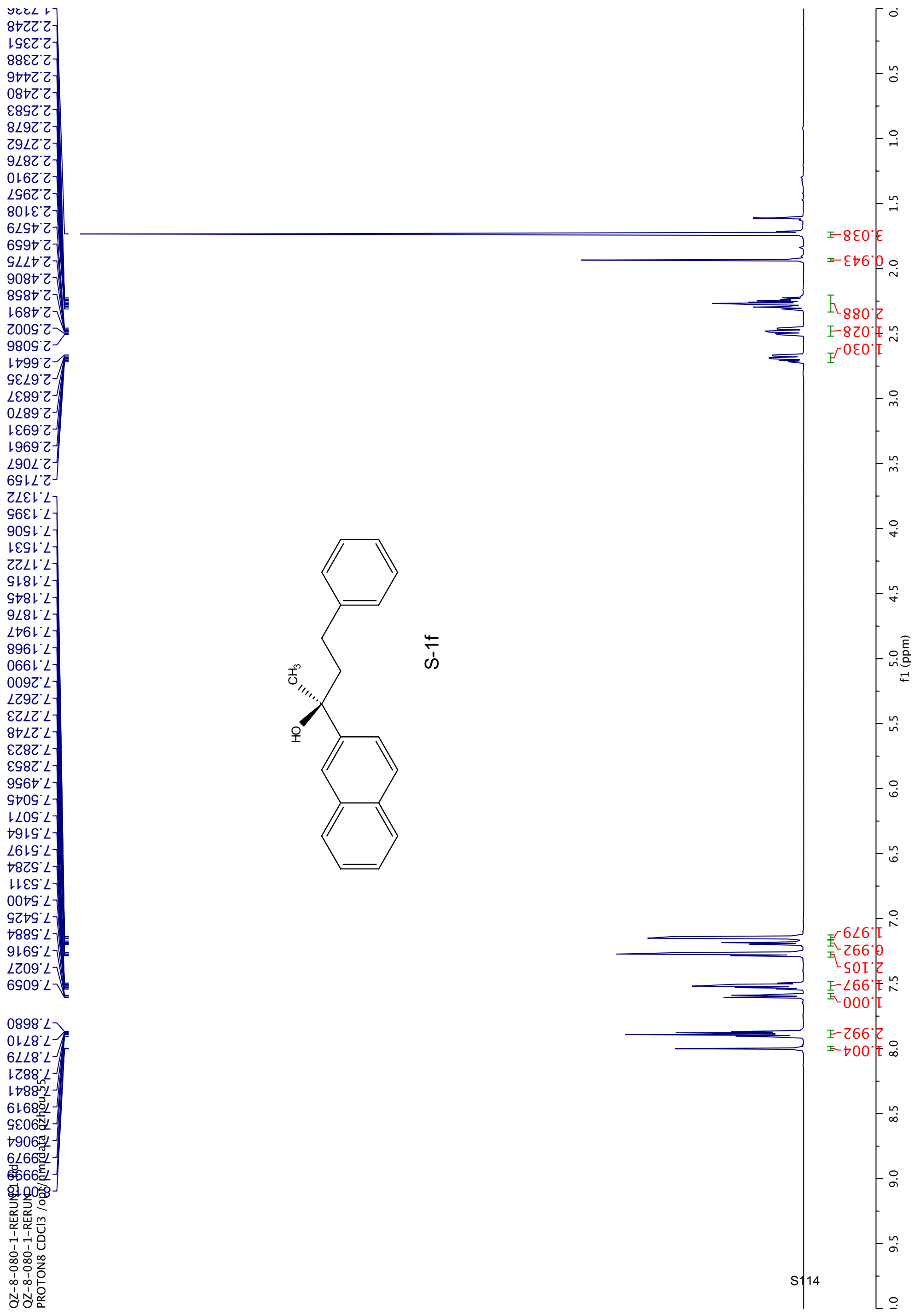


$0 \varepsilon 99^{\circ} 0 \varepsilon_{7}$

$\angle 98 L^{\circ} 0 \mathcal{E}^{-}$

$9668^{\circ}$ G -

0990 $9 L-$ ยІวดว 009l'LL-

$\angle \varepsilon 9 \varepsilon^{\circ} \varepsilon Z L$

†เ89.๕乙レ

乙688'G乙L

EL06.9ZL

عOSZ'9ZL

†0\&9 $\angle Z L]$

9GLL8ZL]=

†68Z'8Zレ

Z997.8ZL-

8ELG'8ZL

6ฤてヤ゙てદレ

LOGE'EEl

乙てォとててャレ一

6ル0งカー
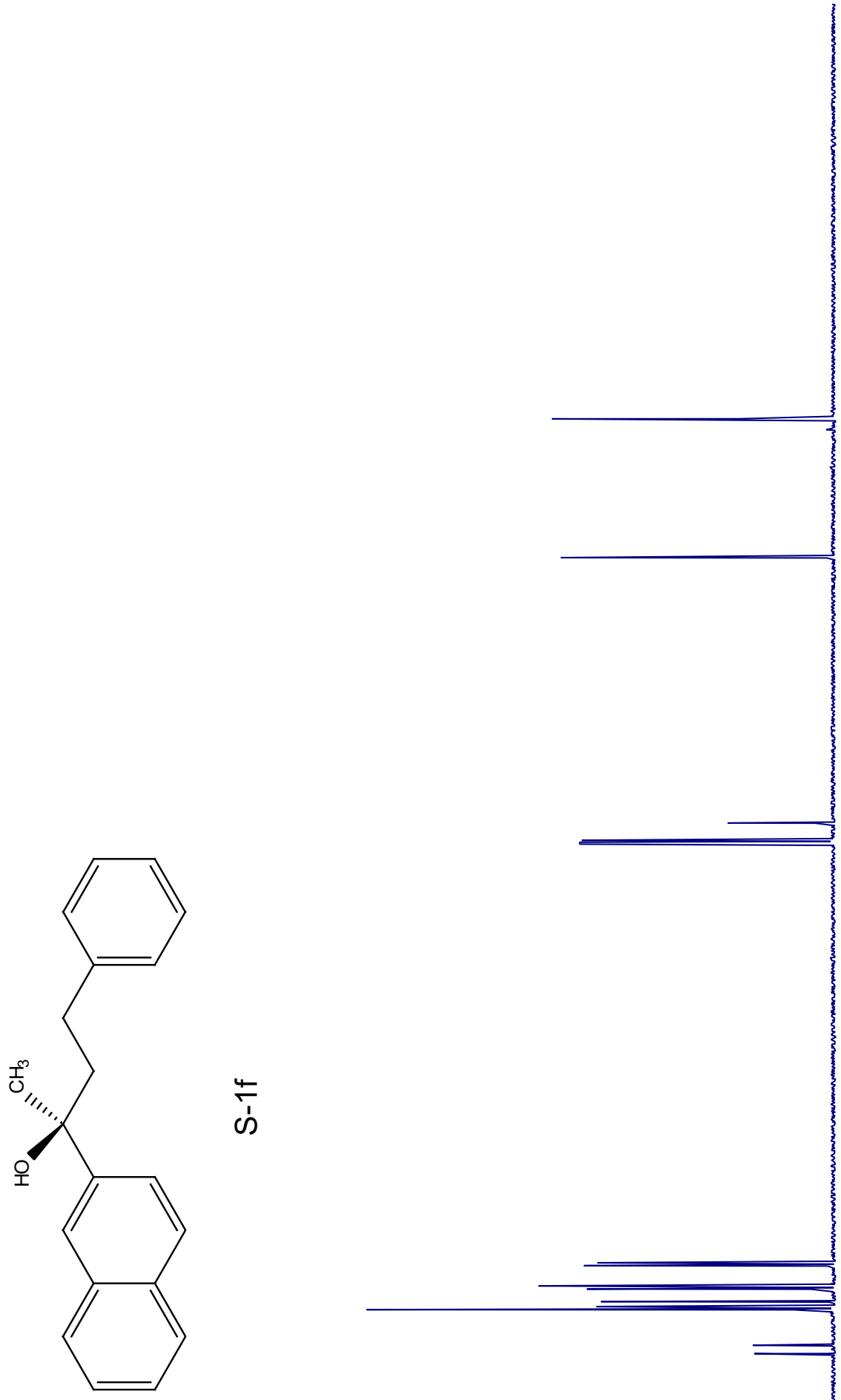


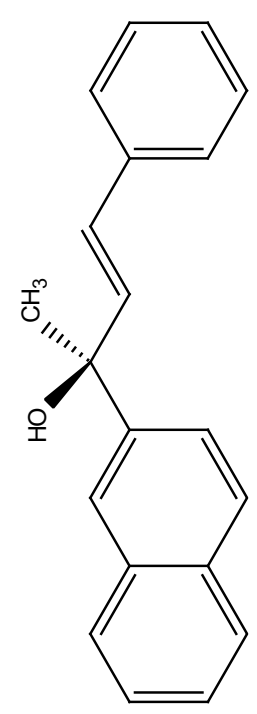


0GSG'E乙レ

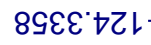
8L90.9ZL †0L乙'9ZL L6L L'9ZL

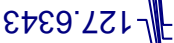
60Z8 LZレ 乙E9L:8ZL 0ᄂ乙Z 8ZLก&6Zع 8ZL ○9L乙L8ZL

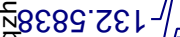

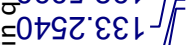

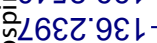

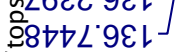

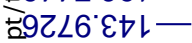

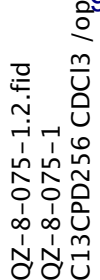
$\overline{\mathrm{m}}$
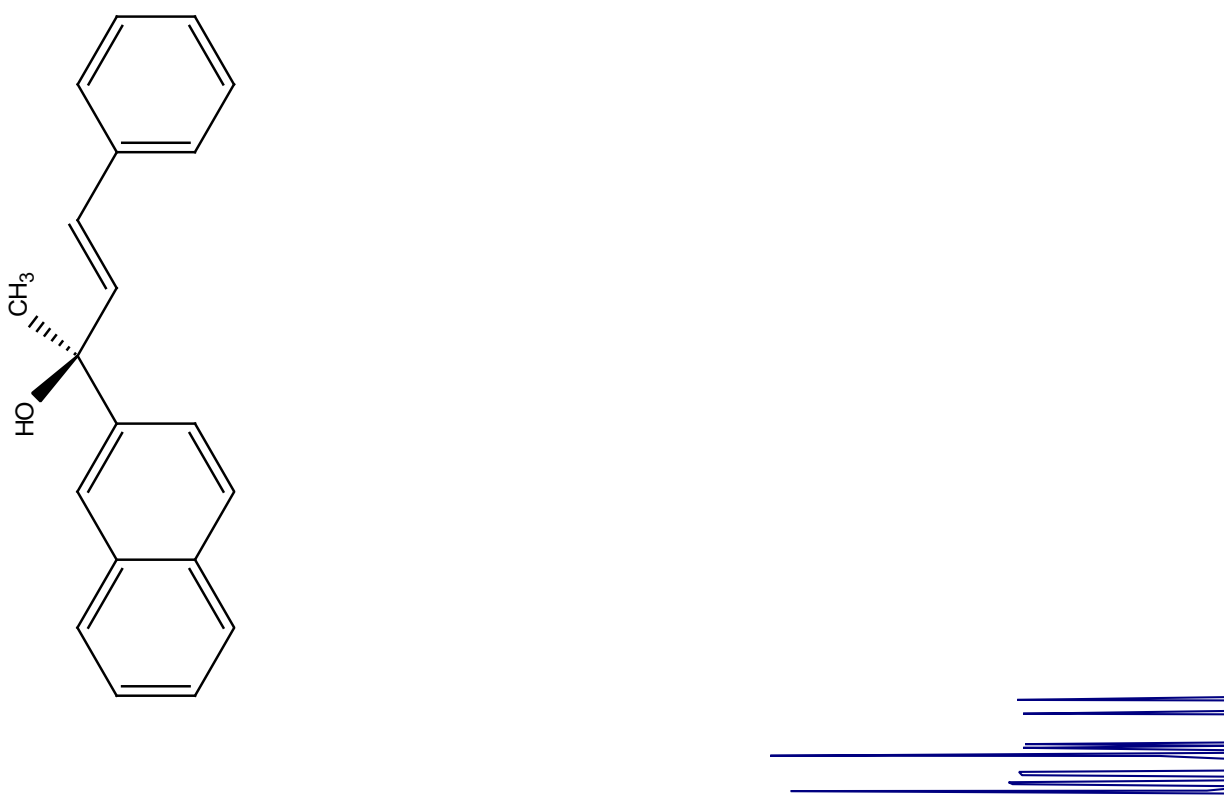

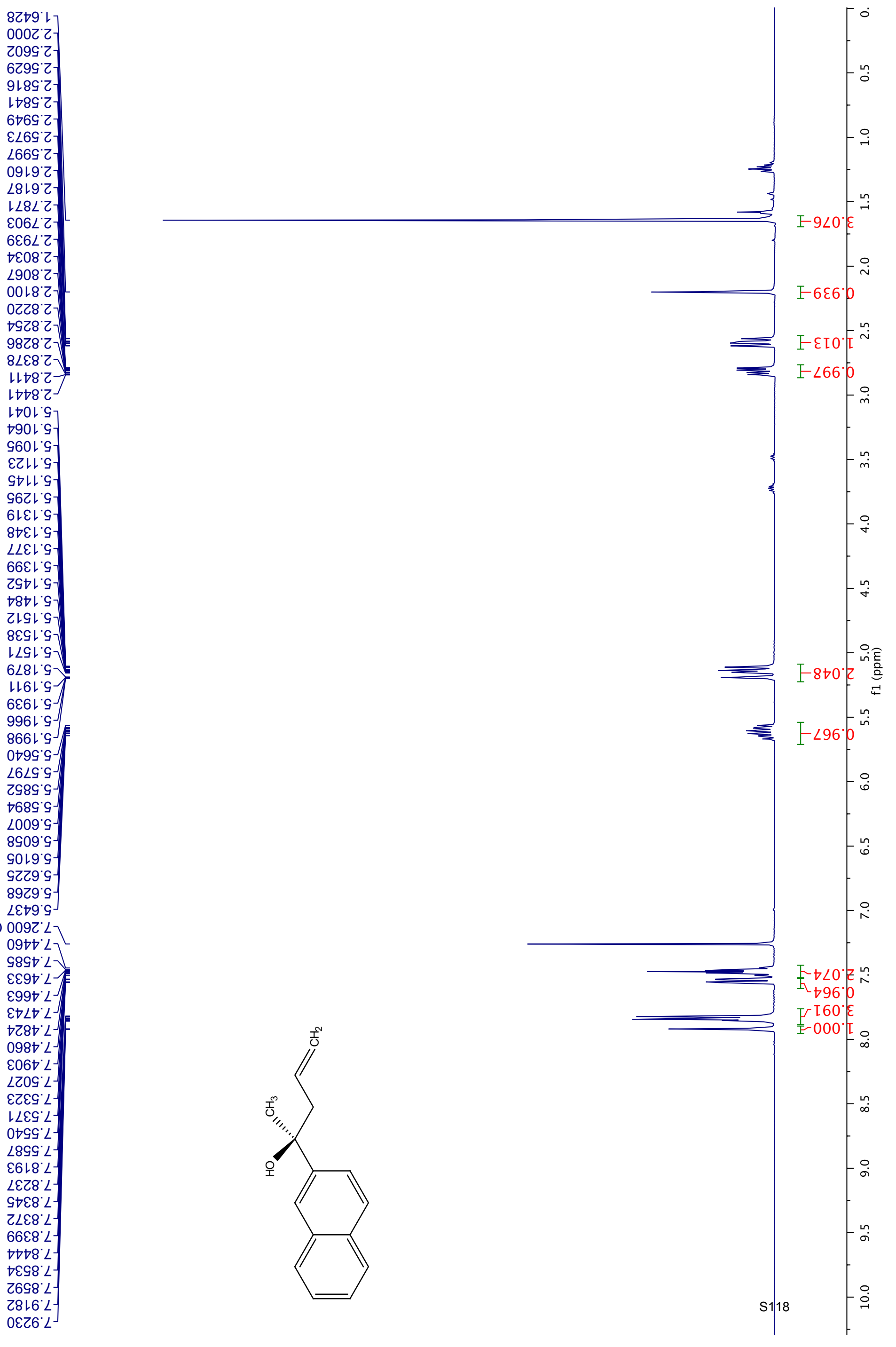
L9ع8'6L 0GZЕ'EZレ SGL9EZL] Z998.9ZL] Z60Z 9ZL] $9709^{\circ} \mathrm{LZ}$ เ6ヤ0.8Zレ GELZ'8Z! L6GE'乙EL9LL乙'EEL -

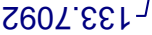

66L0`ฺレー

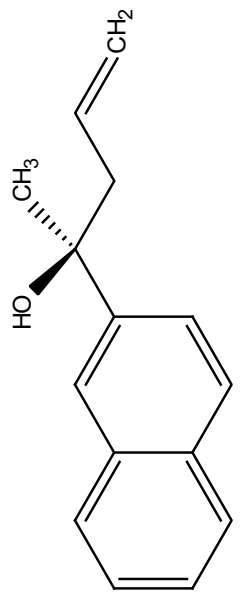



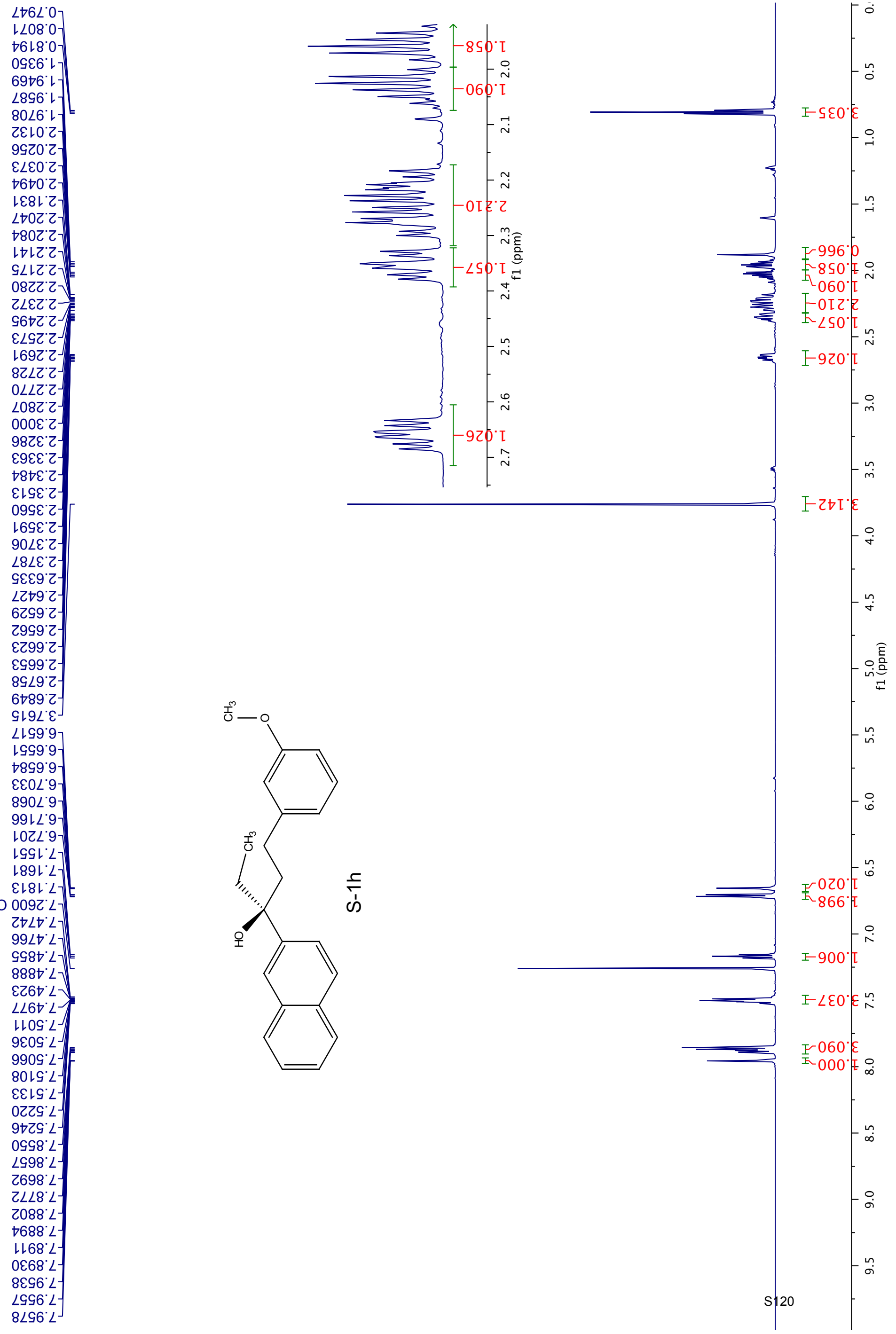
${ }^{\top} 906^{\circ} L-$

$8 † 0 \varepsilon^{\circ} 0 \varepsilon-$

9088'ฐะ-

ててムガャカー

†6ะ乙 ૬૬-

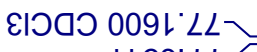

ILE9 $L L$

OSEZ゙ルレー

ธع0Z๋レー

90\&8.0ZL

69Z6 हZL

$\left.\angle เ 9 \varepsilon^{\circ} \triangleright Z L\right][$

Z608'GZL]

8E8เ $92 \mathrm{~L}$

StLGLZL-

$\checkmark \angle 80{ }^{\circ} 8 Z$ -

sL9Z 8ZL

$0 \nabla \angle t 6 Z L][$

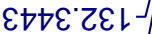

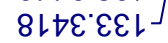
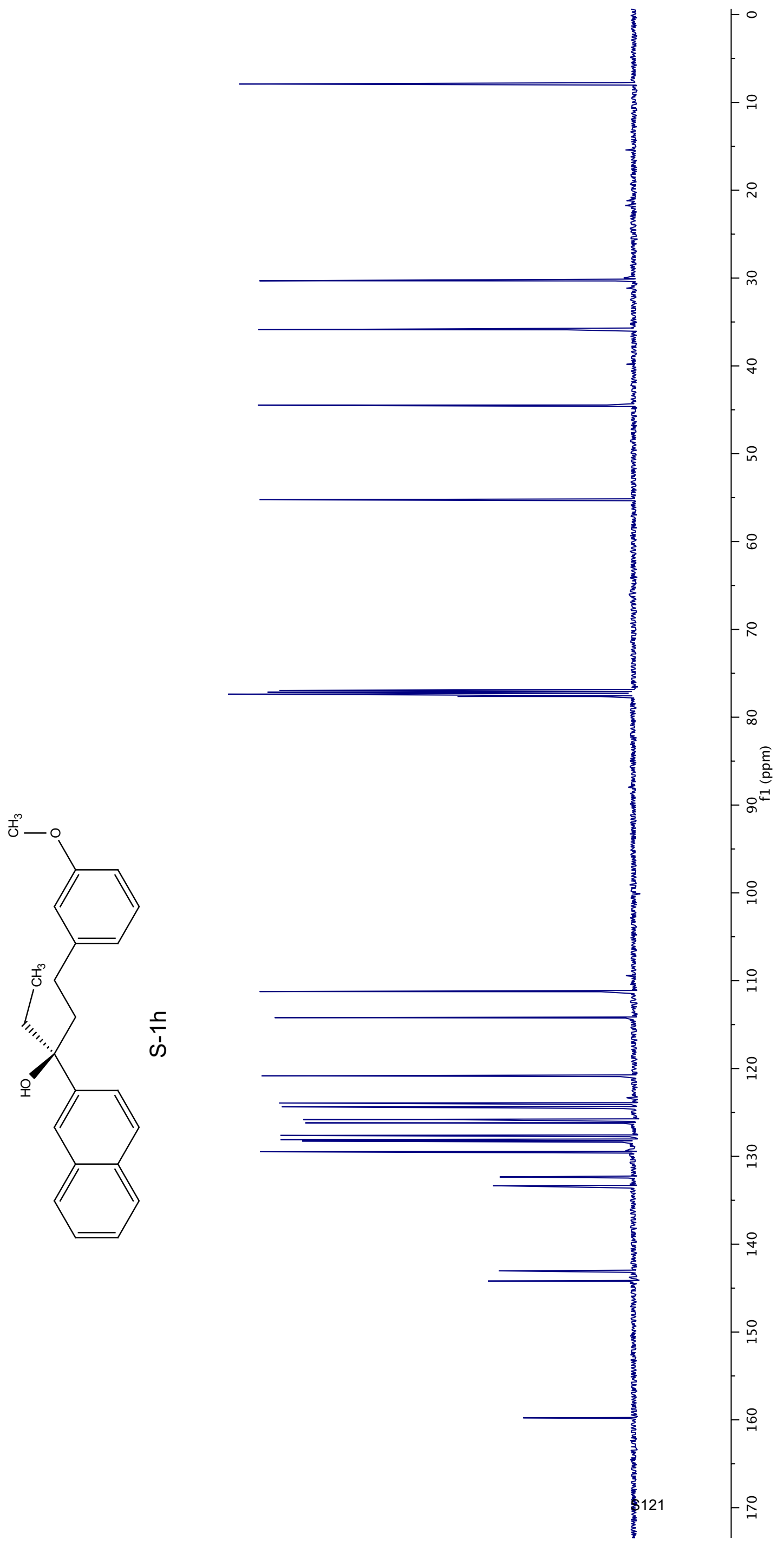

m $\varepsilon s \varepsilon 0^{\circ} \varepsilon t レ$

2レ6เ十tレ

$8+9 L^{\prime} 69 L-$

I

I 


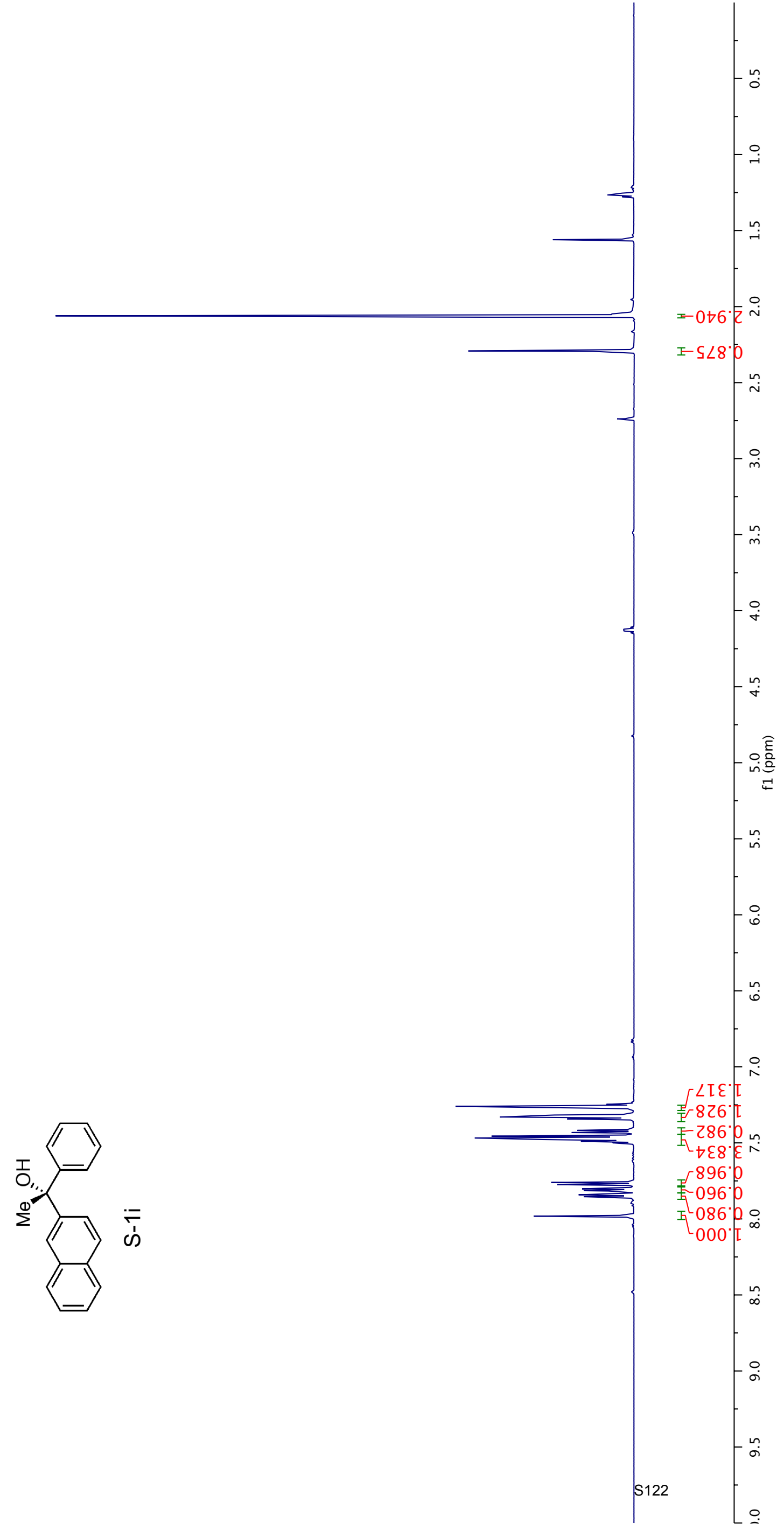


t9l9. 9 L हागهว 009. LL

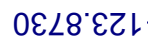

ELLO'GZL

6ع60.9ZL

E660"9ZL

88L乙'9ZL

$980{ }^{\circ} \angle Z L$

$\left.\triangleright 8 \varepsilon 9^{\circ} \angle Z L\right]$

EOL'8ZL

$\rightarrow \angle \varepsilon^{\circ} 8 Z L$

เ96ย'8ZL

ナ 90EG'ZEL

$66 \varepsilon$ ' $^{\circ \varepsilon L}-$
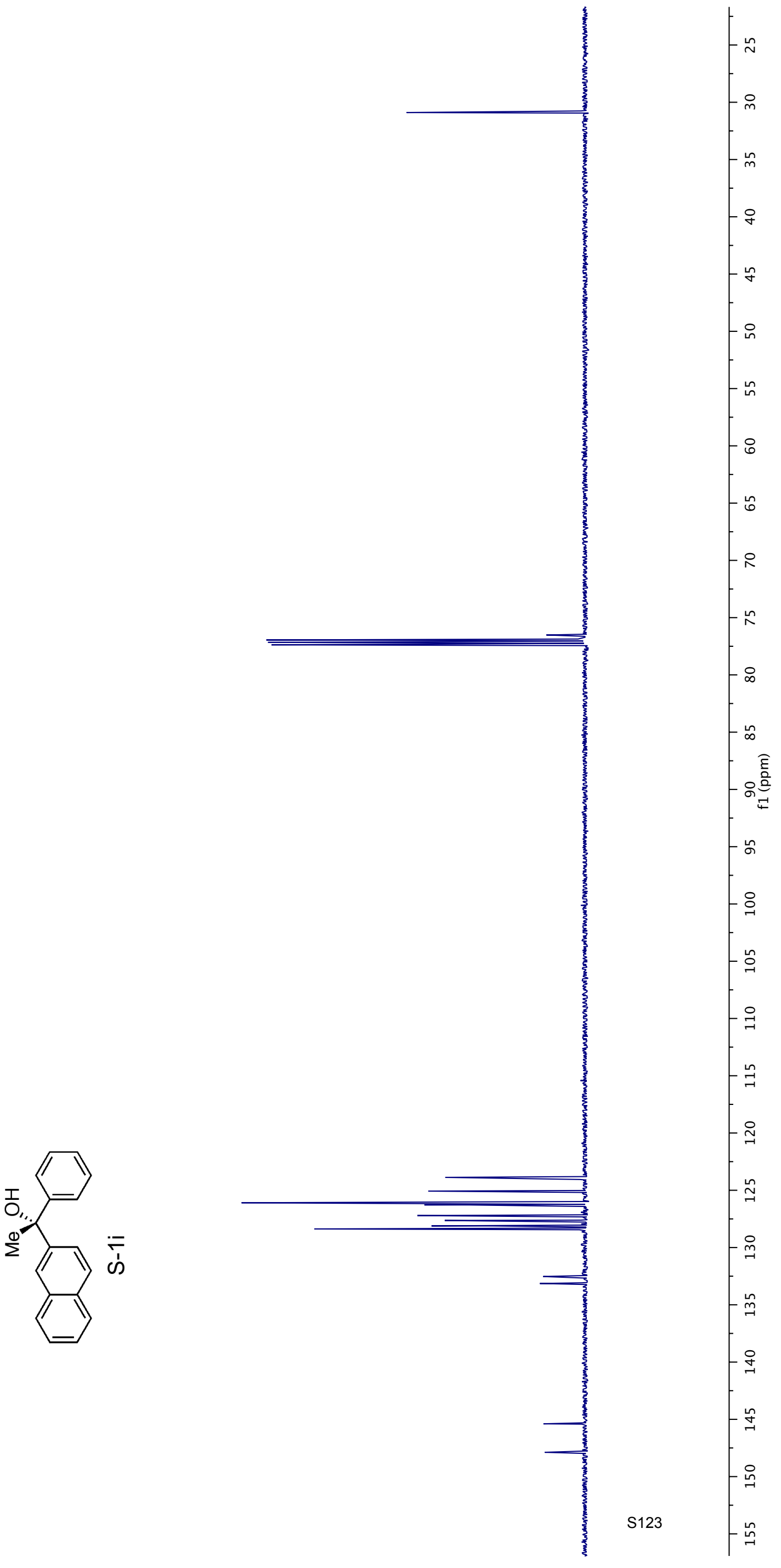


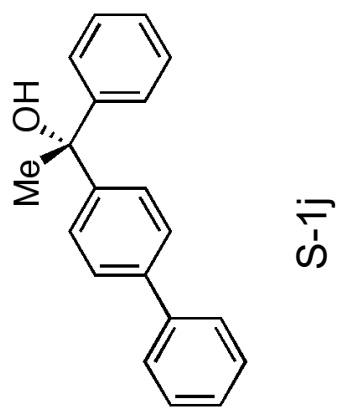


ย9ع0.เE-

G692.9L

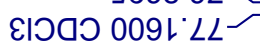

$\nabla \nabla \angle 6$ GZL

L8Zt 9ZL

$\left.1090^{\circ} \angle Z L\right]$

$\angle Z \angle L^{\circ} \angle Z L$

$080 Z$ LZL-

†96ย' LZL-

8†LE'8ZL

\&†88.8Z1

Z†96.6ع -

E6L80レー

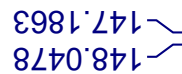

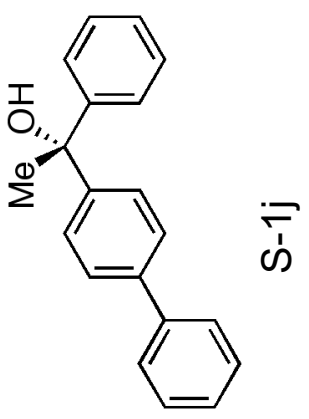


Racemic 4

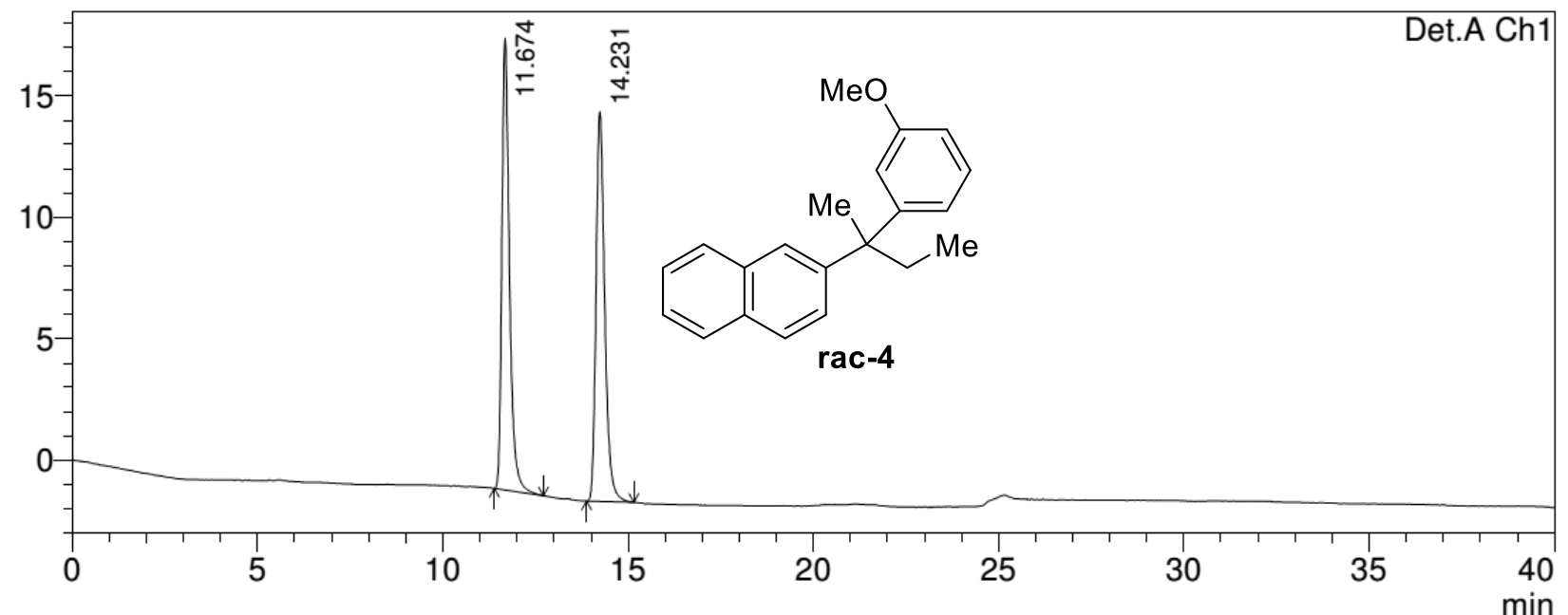

Detector A Ch1 254nm

\begin{tabular}{|r|r|r|r|r|r|}
\hline \multicolumn{1}{|c|}{ Peak\# } & Ret. Time & Area & Height & Area \% & Height \% \\
\hline 1 & 11.674 & 264030 & 18609 & 50.155 & 53.657 \\
\hline 2 & 14.231 & 262397 & 16073 & 49.845 & 46.343 \\
\hline Total & & 526427 & 34682 & 100.000 & 100.000 \\
\hline
\end{tabular}

Enantioenriched 4, 93\% ee

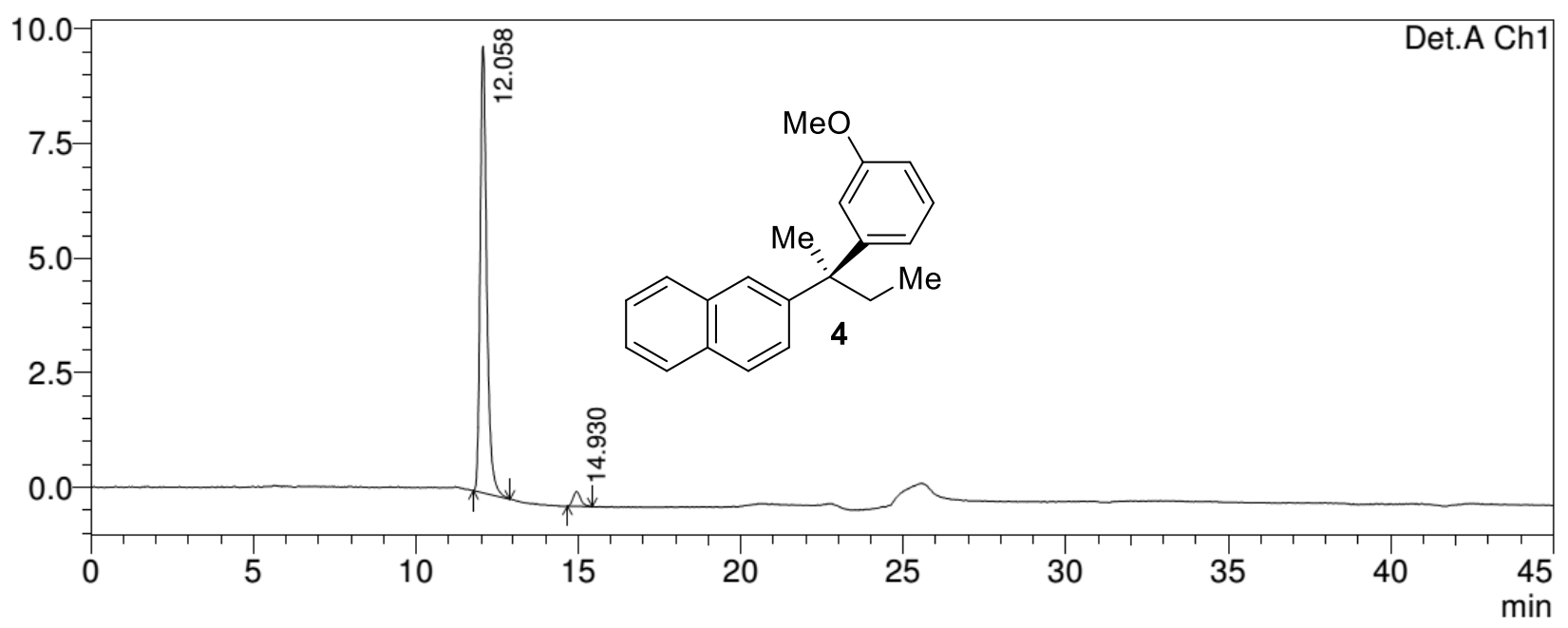

Detector A Ch1 254nm

\begin{tabular}{|r|r|r|r|r|r|}
\hline \multicolumn{1}{|c|}{ Peak\# } & Ret. Time & \multicolumn{1}{|c|}{ Area } & \multicolumn{1}{|c|}{ Height } & Area \% & Height \% \\
\hline 1 & 12.058 & 138560 & 9748 & 96.319 & 96.759 \\
\hline 2 & 14.930 & 5295 & 326 & 3.681 & 3.241 \\
\hline Total & & 143855 & 10075 & 100.000 & 100.000 \\
\hline
\end{tabular}


Racemic 6

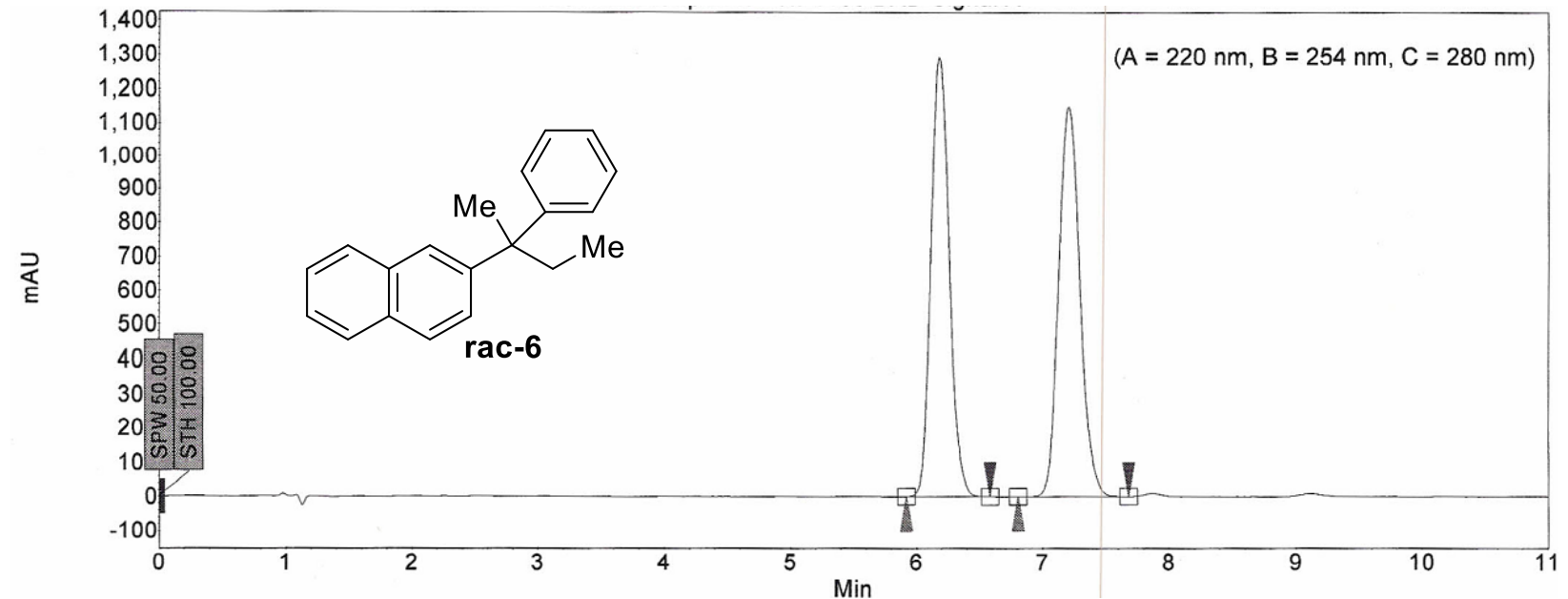

\begin{tabular}{|c|l|r|r|r|r|r|r|r|r|}
\hline Index & Name & Start & Time & End & RT Offset & Quantity & Height & Area & Area \\
\hline \hline & & {$[\mathrm{Min}]$} & {$[\mathrm{Min}]$} & {$[\mathrm{Min}]$} & {$[\mathrm{Min}]$} & {$[\%$ Area] } & {$[\mu \mathrm{V}]$} & {$[\mu \mathrm{V} . \mathrm{Min}]$} & {$[\%]$} \\
\hline 1 & UNKNOWN & 5.92 & 6.18 & 6.58 & 0.00 & 49.41 & 1292.6 & 217.5 & 49.413 \\
\hline 2 & UNKNOWN & 6.81 & 7.21 & 7.68 & 0.00 & 50.59 & 1148.9 & 222.7 & 50.587 \\
\hline & & & & & & & & & \\
\hline Total & & & & & & 100.00 & 2441.5 & 440.2 & 100.000 \\
\hline
\end{tabular}

Enantioenriched 6, 95\% ee

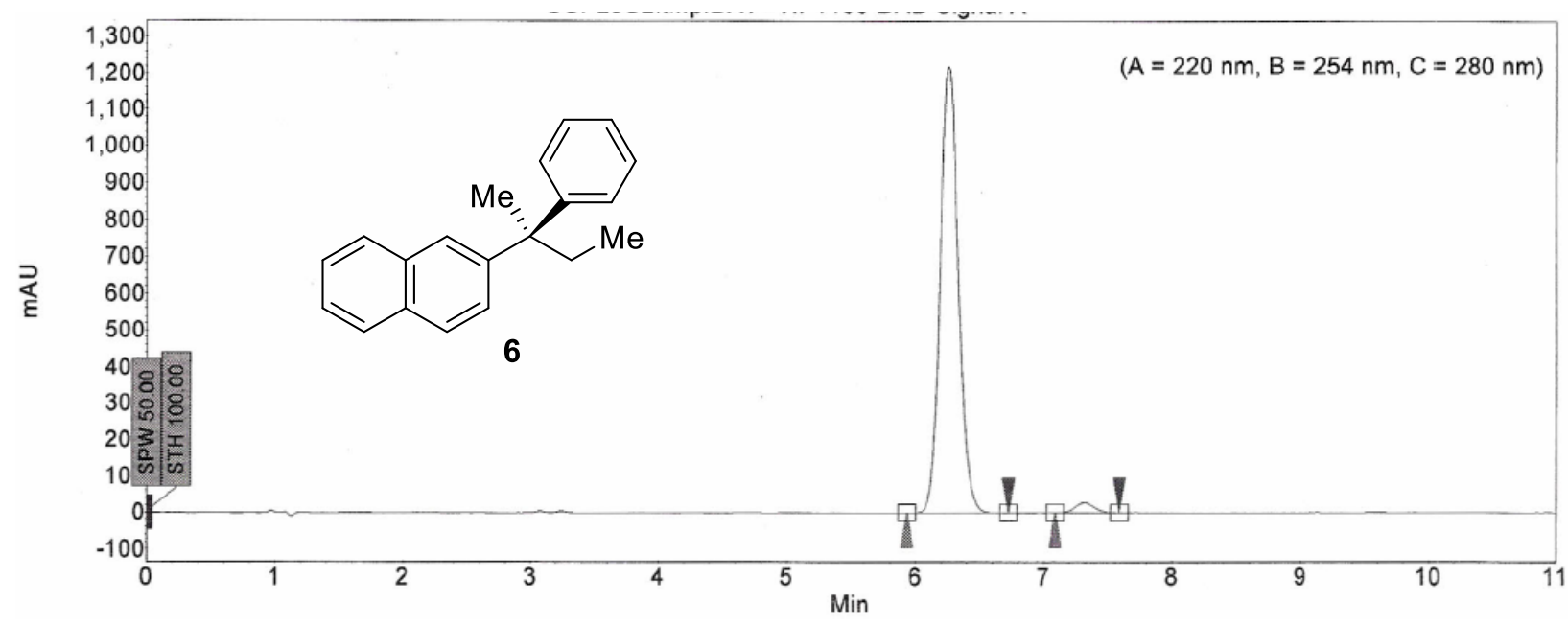

\begin{tabular}{|c|l|r|r|r|r|r|r|r|r|}
\hline Index & Name & Start & Time & End & RT Offset & Quantity & Height & Area & Area \\
\hline \hline & & {$[\mathrm{Min}]$} & {$[\mathrm{Min}]$} & {$[\mathrm{Min}]$} & {$[\mathrm{Min}]$} & {$[\%$ Area] } & {$[\mu \mathrm{V}]$} & {$[\mu \mathrm{V} . \mathrm{Min}]$} & {$[\%]$} \\
\hline 2 & UNKNOWN & 5.93 & 6.25 & 6.73 & 0.00 & 97.62 & 1220.1 & 202.1 & 97.625 \\
\hline 1 & UNKNOWN & 7.09 & 7.32 & 7.59 & 0.00 & 2.38 & 27.5 & 4.9 & 2.375 \\
\hline & & & & & & & & & \\
\hline Total & & & & & & 100.00 & 1247.6 & 207.0 & 100.000 \\
\hline
\end{tabular}


Racemic 7

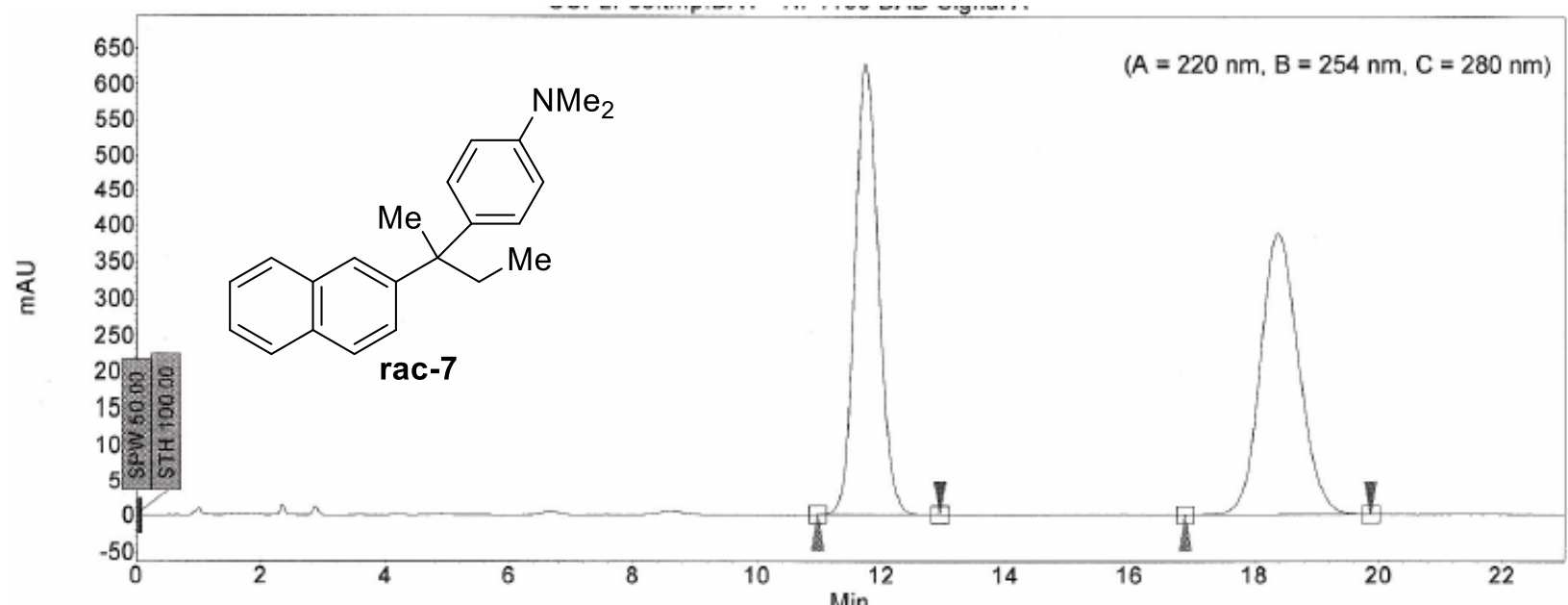

\begin{tabular}{|c|l|r|r|r|r|r|r|r|r|}
\hline Index & Name & Start & Time & End & RT Offset & Quantity & Height & Area & Area \\
\hline & & {$[\mathrm{Min}]$} & {$[\mathrm{Min}]$} & {$[\mathrm{Min}]$} & {$[\mathrm{Min}]$} & {$[\%$ Area] } & {$[\mu \mathrm{V}]$} & {$[\mu \mathrm{V} . \mathrm{Min}]$} & {$[\%]$} \\
\hline 1 & UNKNOWN & 10.97 & 11.74 & 12.94 & 0.00 & 49.90 & 627.8 & 276.5 & 49.904 \\
\hline 2 & UNKNOWN & 16.89 & 18.37 & 19.87 & 0.00 & 50.10 & 387.5 & 277.5 & 50.096 \\
\hline & & & & & & & & & \\
\hline Total & & & & & & 100.00 & 1015.2 & 554.0 & 100.000
\end{tabular}

Enantioenriched 7, 96\% ee

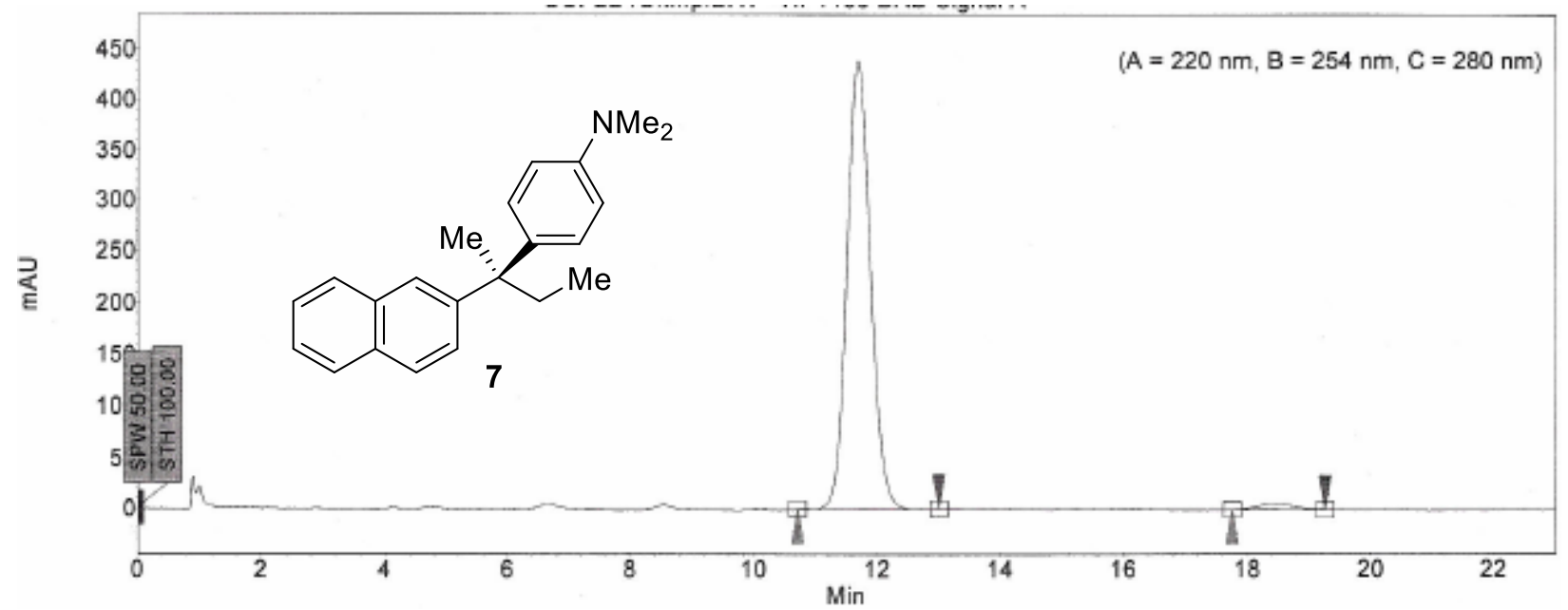

\begin{tabular}{|c|l|r|r|r|r|r|r|r|r|}
\hline Index & Name & Start & Time & End & RT Offset & Quantity & Height & Area & Area \\
\hline \hline & & {$[\mathrm{Min}]$} & {$[\mathrm{Min}]$} & {$[\mathrm{Min}]$} & {$[\mathrm{Min}]$} & {$[\%$ Area] } & {$[\mu \mathrm{V}]$} & {$[\mathrm{\mu V}, \mathrm{Min}]$} & {$[\%]$} \\
\hline 1 & UNKNOWN & 10.71 & 11.68 & 13.00 & 0.00 & 97.91 & 439.2 & 193.4 & 97.908 \\
\hline 2 & UNKNOWN & 17.76 & 18.49 & 19.25 & 0.00 & 2.09 & 6.1 & 4.1 & 2.092 \\
\hline & & & & & & & & & \\
\hline Total & & & & & & 100.00 & 445.4 & 197.5 & 100.000 \\
\hline
\end{tabular}


Racemic 8

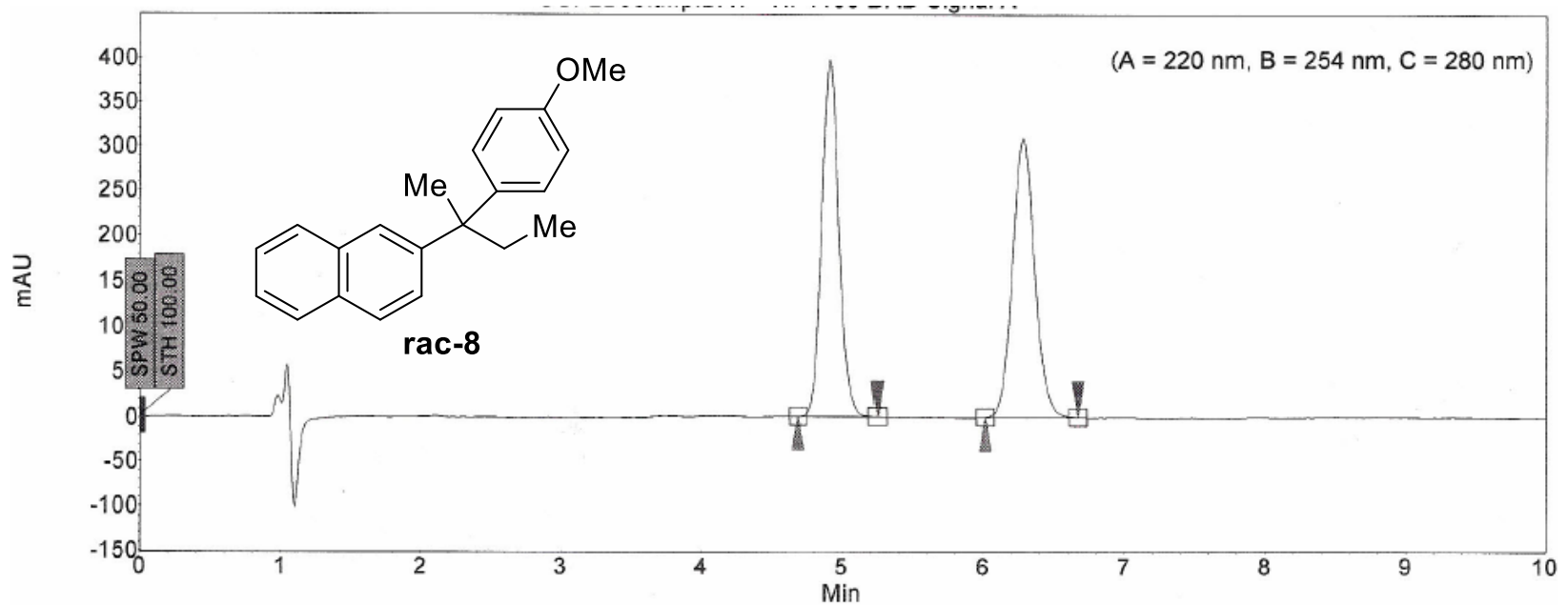

\begin{tabular}{|c|l|r|r|r|r|r|r|r|r|}
\hline Index & Name & Start & Time & End & RT Offset & Quantity & Height & Area & Area \\
\hline \hline & & {$[\mathrm{Min}]$} & {$[\mathrm{Min}]$} & {$[\mathrm{Min}]$} & {$[\mathrm{Min}]$} & {$[\%$ Area] } & {$[\mu \mathrm{V}]$} & {$[\mu \mathrm{V} . \mathrm{Min}]$} & {$[\%]$} \\
\hline 1 & UNKNOWN & 4.69 & 4.91 & 5.25 & 0.00 & 49.78 & 396.5 & 55.7 & 49.783 \\
\hline 2 & UNKNOWN & 6.01 & 6.28 & 6.67 & 0.00 & 50.22 & 308.1 & 56.2 & 50.217 \\
\hline & & & & & & & & & \\
\hline Total & & & & & & 100.00 & 704.6 & 112.0 & 100.000 \\
\hline
\end{tabular}

Enantioenriched 8, 96\% ee

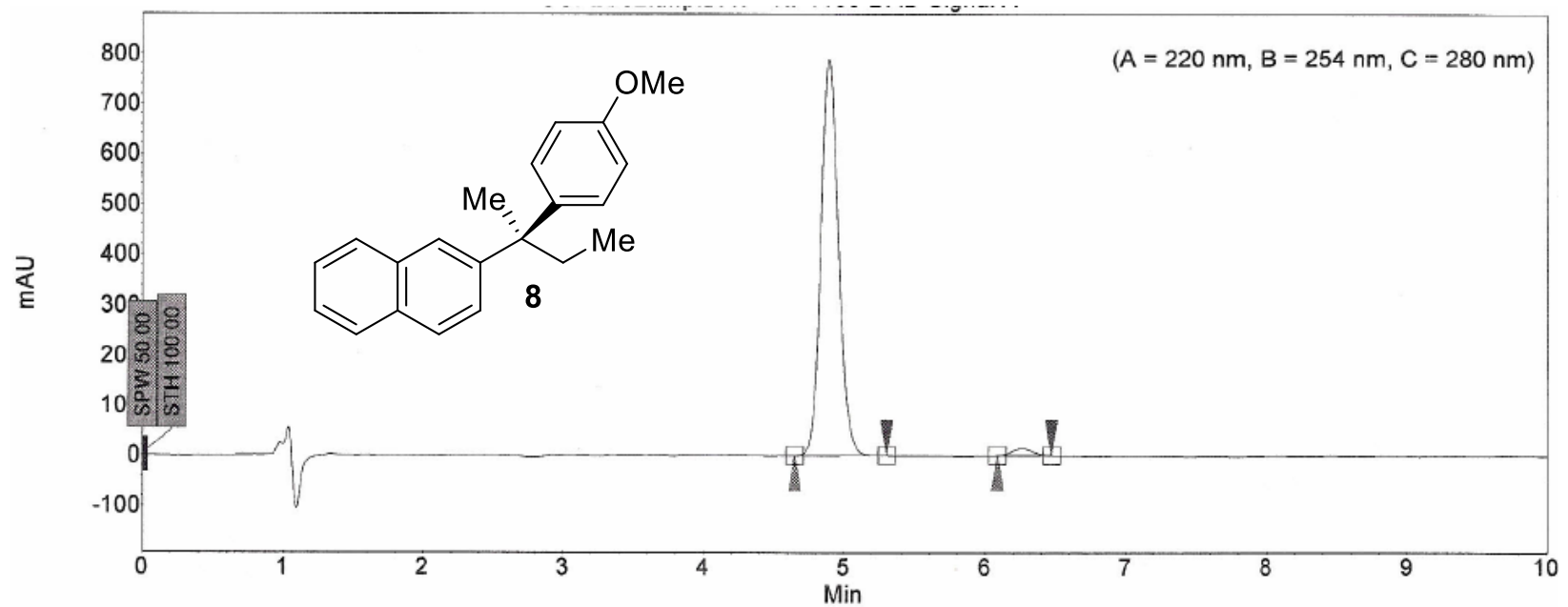

\begin{tabular}{|c|l|r|r|r|r|r|r|r|r|}
\hline Index & Name & Start & Time & End & RT Offset & Quantity & Height & Area & Area \\
\hline \hline & & {$[\mathrm{Min}]$} & {$[\mathrm{Min}]$} & {$[\mathrm{Min}]$} & {$[\mathrm{Min}]$} & {$[\%$ Area] } & {$[\mu \mathrm{V}]$} & {$[\mu \mathrm{V} . \mathrm{Min}]$} & {$[\%]$} \\
\hline 2 & UNKNOWN & 4.65 & 4.89 & 5.30 & 0.00 & 98.05 & 790.5 & 114.5 & 98.051 \\
\hline 1 & UNKNOWN & 6.09 & 6.27 & 6.47 & 0.00 & 1.95 & 13.5 & 2.3 & 1.949 \\
\hline & & & & & & & & & \\
\hline Total & & & & & & 100.00 & 804.0 & 116.8 & 100.000 \\
\hline
\end{tabular}


Racemic 9

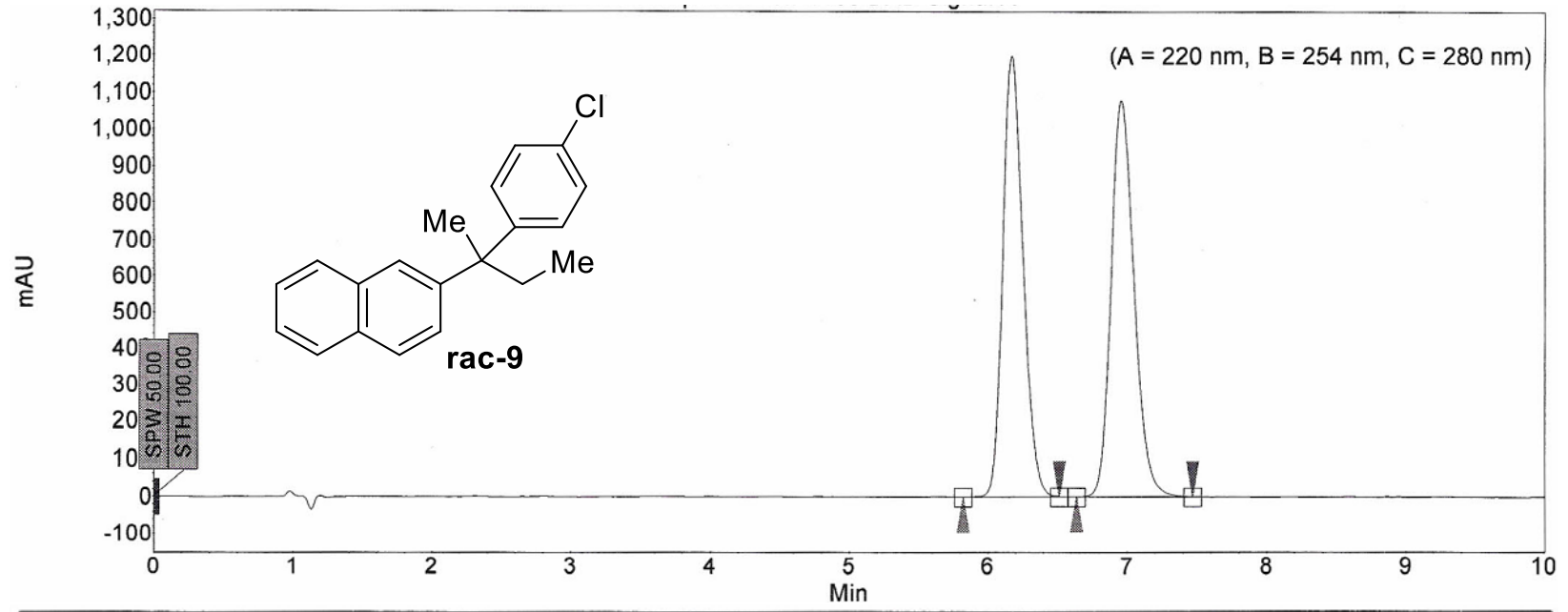

\begin{tabular}{|c|l|r|r|r|r|r|r|r|r|}
\hline Index & Name & Start & Time & End & RT Offset & Quantity & Height & Area & Area \\
\hline \hline & & {$[$ Min] } & {$[$ Min] } & {$[$ Min] } & {$[$ Min] } & {$[\%$ Area] } & {$[\mu \mathrm{V}]$} & {$[\mu \mathrm{V}$. Min] } & {$[\%]$} \\
\hline 1 & UNKNOWN & 5.82 & 6.17 & 6.52 & 0.00 & 49.58 & 1197.4 & 201.8 & 49.580 \\
\hline 2 & UNKNOWN & 6.64 & 6.96 & 7.47 & 0.00 & 50.42 & 1078.7 & 205.2 & 50.420 \\
\hline & & & & & & & & & \\
\hline Total & & & & & & 100.00 & 2276.1 & 407.0 & 100.000 \\
\hline
\end{tabular}

Enantioenriched 9, 92\% ee

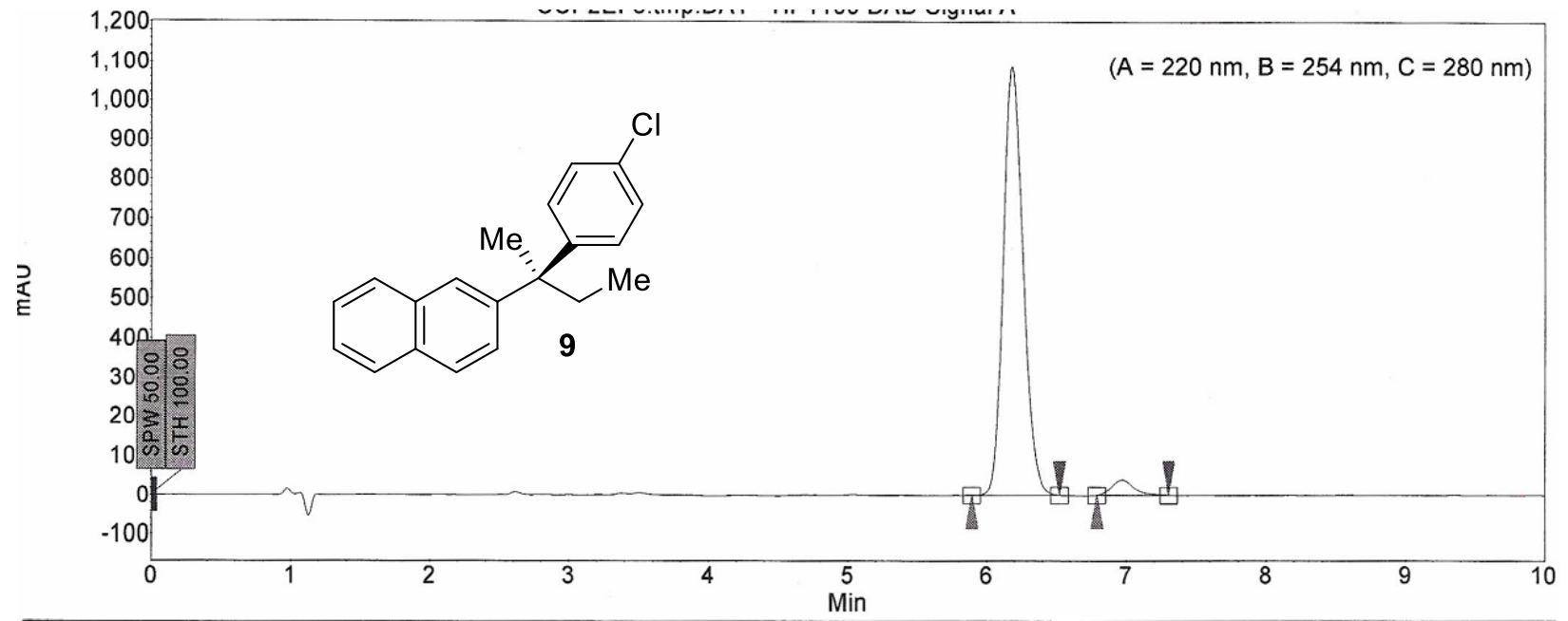

\begin{tabular}{|c|l|r|r|r|r|r|r|r|r|}
\hline Index & Name & Start & Time & End & RT Offset & Quantity & Height & Area & Area \\
\hline \hline & & {$[\mathrm{Min}]$} & {$[\mathrm{Min}]$} & {$[\mathrm{Min}]$} & {$[\mathrm{Min}]$} & {$[\%$ Area] } & {$[\mu \mathrm{V}]$} & {$[\mu \mathrm{V} . \mathrm{Min}]$} & {$[\%]$} \\
\hline 1 & UNKNOWN & 5.89 & 6.18 & 6.52 & 0.00 & 96.01 & 1087.1 & 180.0 & 96.009 \\
\hline 2 & UNKNOWN & 6.79 & 6.97 & 7.30 & 0.00 & 3.99 & 41.2 & 7.5 & 3.991 \\
\hline & & & & & & & & & \\
\hline Total & & & & & & 100.00 & 1128.3 & 187.4 & 100.000 \\
\hline
\end{tabular}


Racemic 10

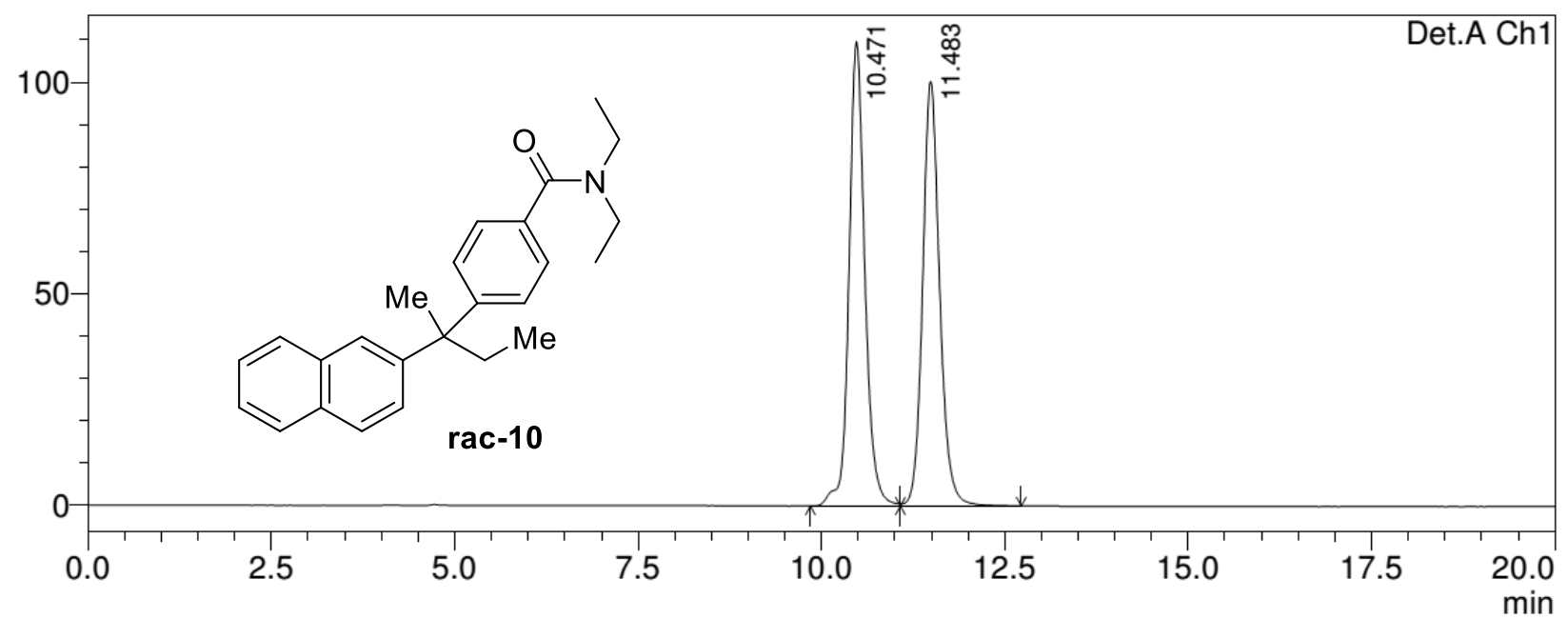

Detector A Ch1 254nm

\begin{tabular}{|r|r|r|r|r|r|}
\hline \multicolumn{1}{|c|}{ Peak\# } & Ret. Time & Area & Height & Area \% & \multicolumn{1}{|c|}{ Height \% } \\
\hline 1 & 10.471 & 1653101 & 109872 & 50.507 & 52.247 \\
\hline 2 & 11.483 & 1619926 & 100424 & 49.493 & 47.753 \\
\hline Total & & 3273026 & 210296 & 100.000 & 100.000 \\
\hline
\end{tabular}

Enantioenriched 10, 94\% ee

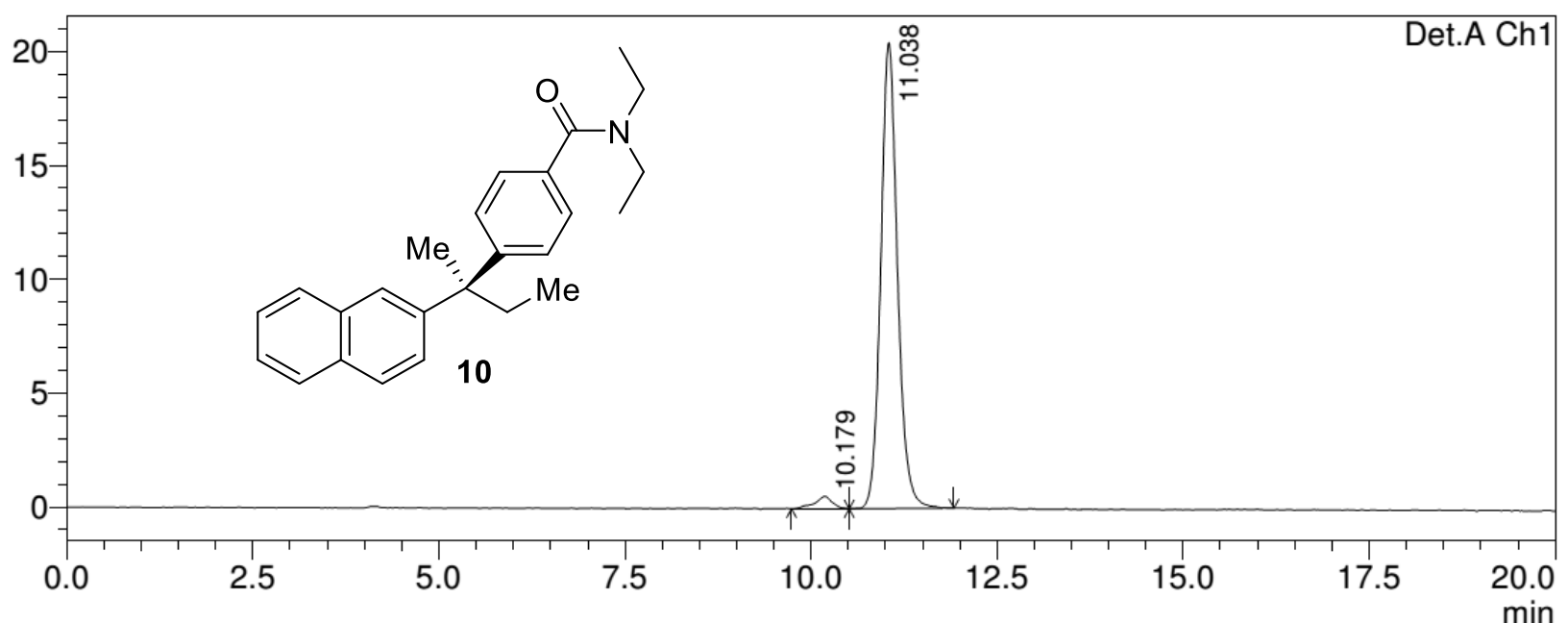

Detector A Chl 254nm

\begin{tabular}{|r|r|r|r|r|r|}
\hline \multicolumn{1}{|c|}{ Peak\# } & \multicolumn{1}{c|}{ Ret. Time } & \multicolumn{1}{c|}{ Area } & \multicolumn{1}{c|}{ Height } & Area \% & \multicolumn{1}{c|}{ Height \% } \\
\hline 1 & 10.179 & 9333 & 548 & 2.832 & 2.609 \\
\hline 2 & 11.038 & 320214 & 20472 & 97.168 & 97.391 \\
\hline Total & & 329548 & 21021 & 100.000 & 100.000 \\
\hline
\end{tabular}


Enantioenriched $(R)-\mathbf{1 0}, 96 \%$ ee (crystal used for X-ray diffraction analysis)

$\mathrm{mAU}$

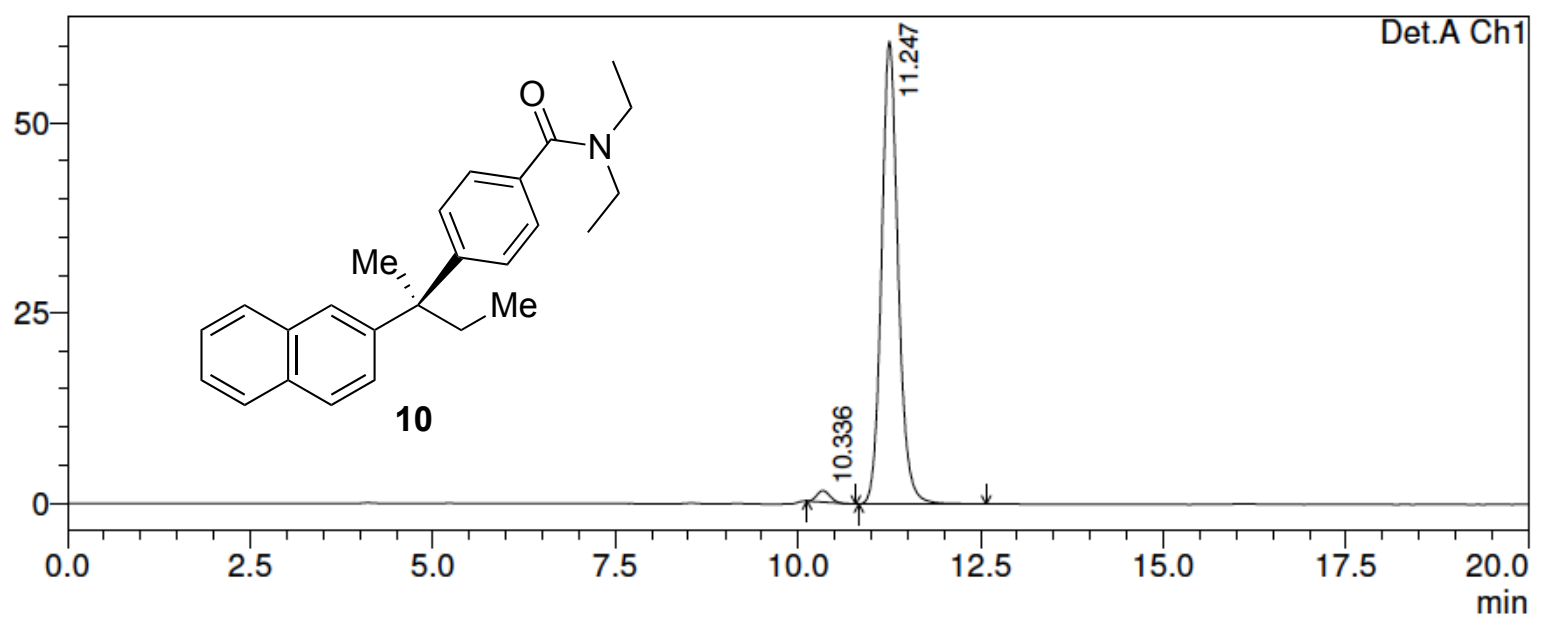

Detector A Ch1 254nm

\begin{tabular}{|r|r|r|r|r|r|}
\hline \multicolumn{1}{|c|}{ Peak\# } & Ret. Time & \multicolumn{1}{|c|}{ Area } & \multicolumn{1}{c|}{ Height } & Area \% & Height \% \\
\hline 1 & 10.336 & 17968 & 1480 & 1.829 & 2.376 \\
\hline 2 & 11.247 & 964530 & 60841 & 98.171 & 97.624 \\
\hline Total & & 982498 & 62321 & 100.000 & 100.000 \\
\hline
\end{tabular}


Racemic 11

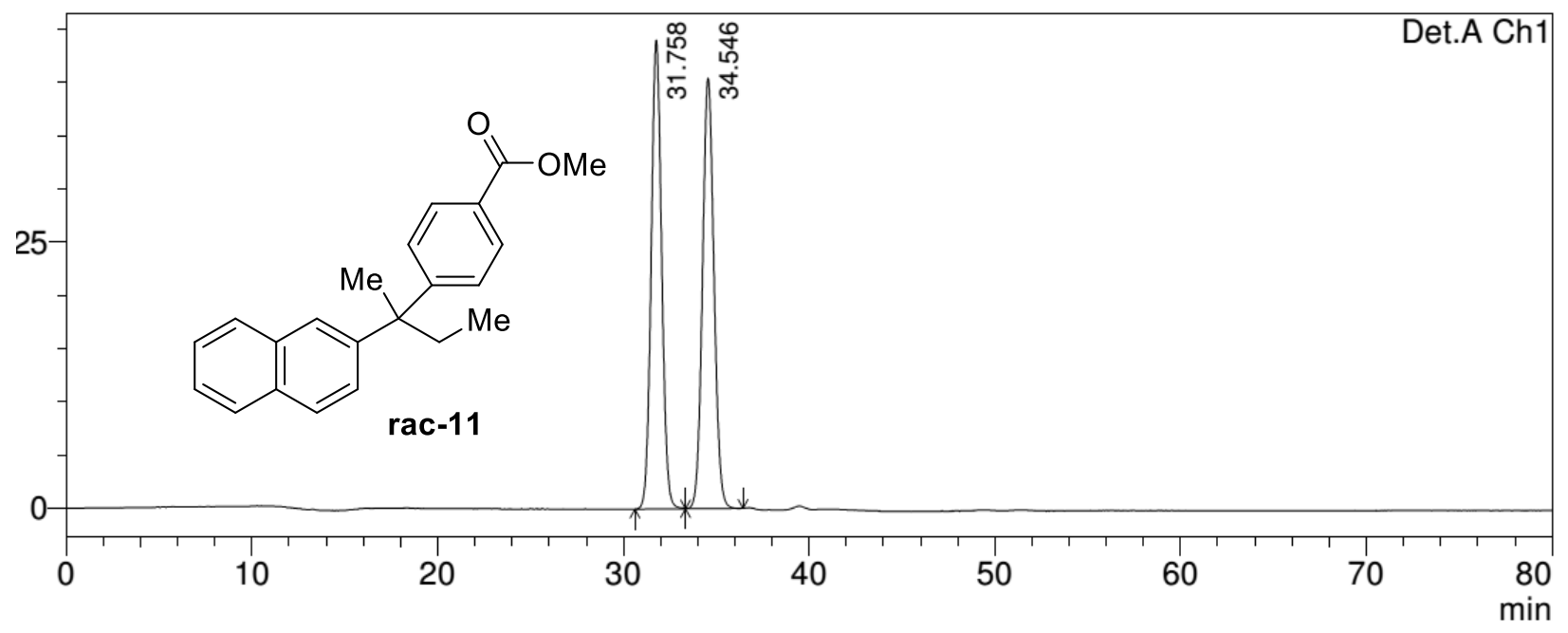

Detector A Ch1 254nm

\begin{tabular}{|r|r|r|r|r|r|}
\hline \multicolumn{1}{|c|}{ Peak\# } & Ret. Time & \multicolumn{1}{c|}{ Area } & Height & Area \% & \multicolumn{1}{c|}{ Height \% } \\
\hline 1 & 31.758 & 1728236 & 44032 & 49.845 & 52.134 \\
\hline 2 & 34.546 & 1738977 & 40427 & 50.155 & 47.866 \\
\hline Total & & 3467213 & 84459 & 100.000 & 100.000 \\
\hline
\end{tabular}

Enantioenriched 11, 96\% ee

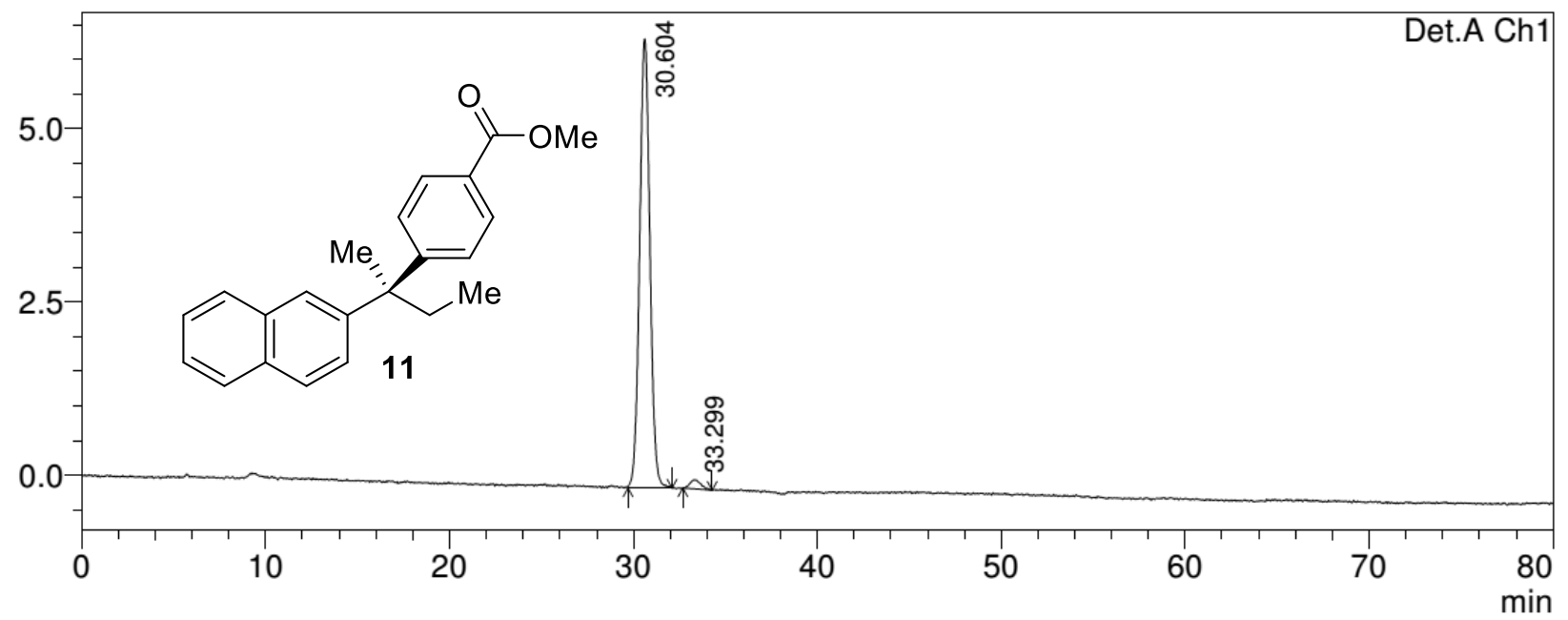

Detector A Ch1 254nm

\begin{tabular}{|r|r|r|r|r|r|}
\hline \multicolumn{1}{|c|}{ Peak\# } & Ret. Time & \multicolumn{1}{c|}{ Area } & \multicolumn{1}{|c|}{ Height } & Area \% & Height \% \\
\hline 1 & 30.604 & 251321 & 6477 & 97.885 & 98.017 \\
\hline 2 & 33.299 & 5430 & 131 & 2.115 & 1.983 \\
\hline Total & & 256751 & 6608 & 100.000 & 100.000 \\
\hline
\end{tabular}


Racemic 12

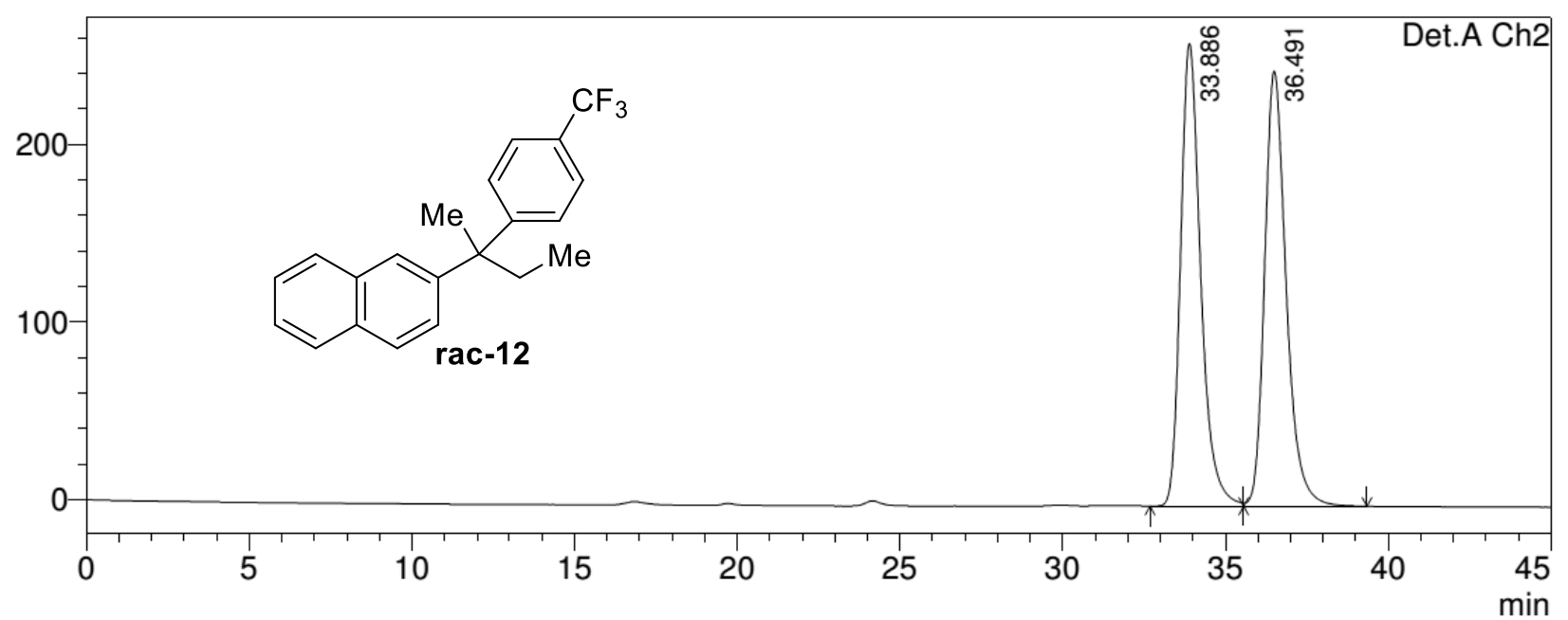

Detector A Ch1 254nm

\begin{tabular}{|r|r|r|r|r|r|}
\hline \multicolumn{1}{|c|}{ Peak\# } & Ret. Time & \multicolumn{1}{|c|}{ Area } & Height & Area \% & \multicolumn{1}{c|}{ Height \% } \\
\hline 1 & 33.885 & 1587788 & 36919 & 49.856 & 51.534 \\
\hline 2 & 36.489 & 1596950 & 34721 & 50.144 & 48.466 \\
\hline Total & & 3184738 & 71640 & 100.000 & 100.000 \\
\hline
\end{tabular}

Enantioenriched 12, 96\% ee

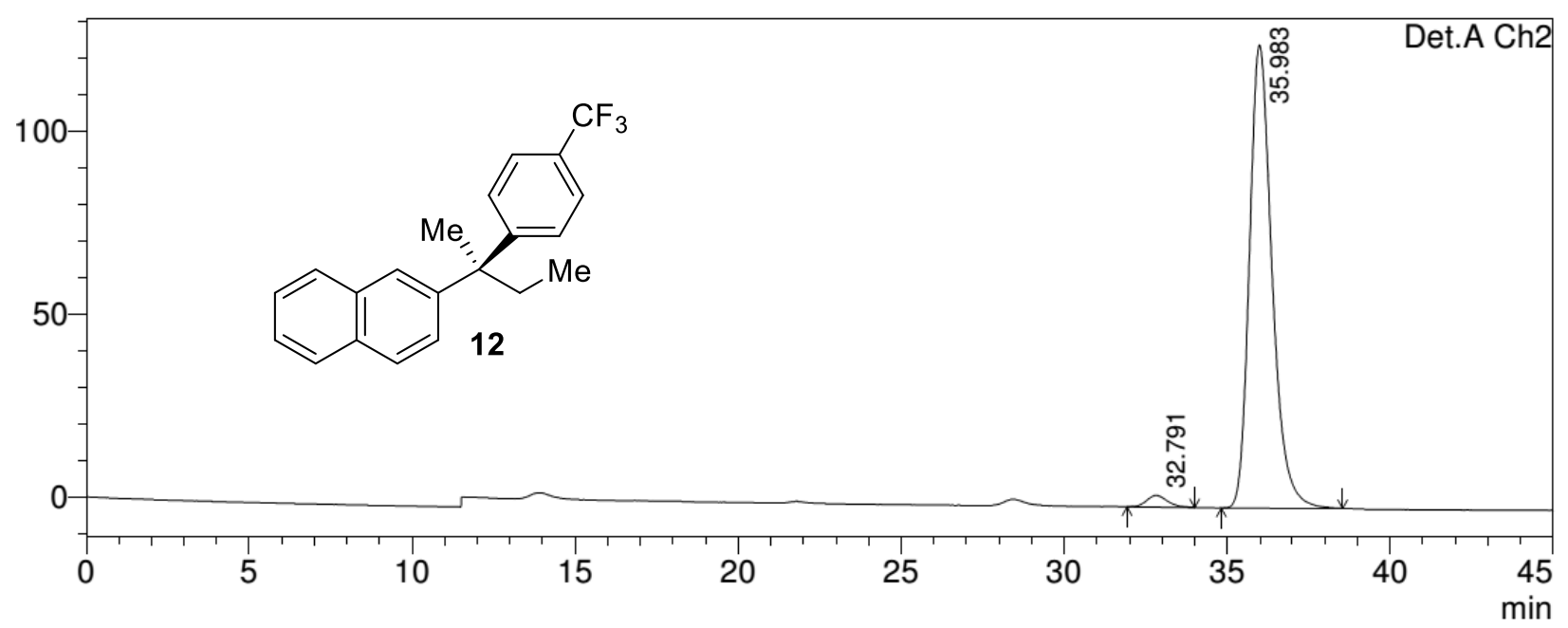

Detector A Ch1 254nm

\begin{tabular}{|r|r|r|r|r|r|}
\hline \multicolumn{1}{|c|}{ Peak\# } & Ret. Time & \multicolumn{1}{c|}{ Area } & \multicolumn{1}{c|}{ Height } & Area \% & \multicolumn{1}{c|}{ Height \% } \\
\hline 1 & 35.980 & 18779 & 442 & 2.148 & 2.424 \\
\hline 2 & 39.173 & 855635 & 17798 & 97.852 & 97.576 \\
\hline Total & & 874414 & 18241 & 100.000 & 100.000 \\
\hline
\end{tabular}


Racemic 13

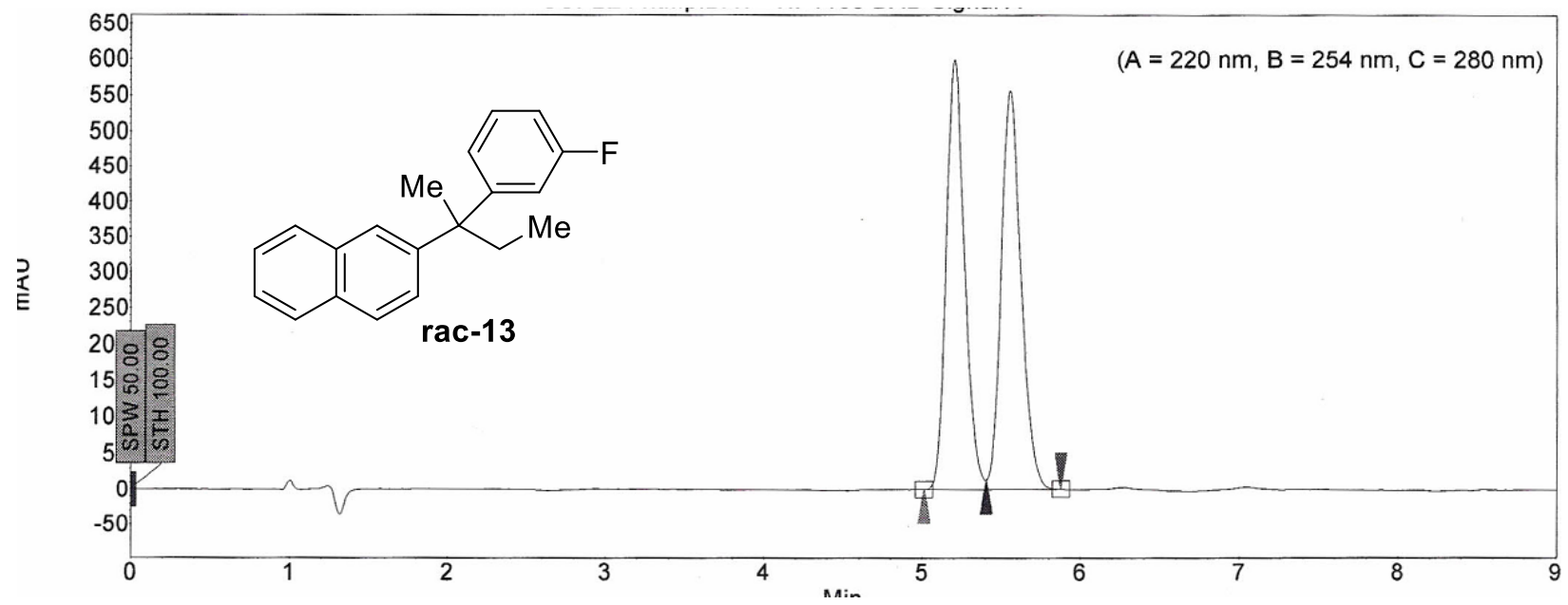

\begin{tabular}{|c|l|r|r|r|r|r|r|r|r|}
\hline Index & Name & Start & Time & End & RT Offset & Quantity & Height & Area & Area \\
\hline \hline & & {$[\mathrm{Min}]$} & {$[\mathrm{Min}]$} & {$[\mathrm{Min}]$} & {$[\mathrm{Min}]$} & {$[\%$ Area] } & {$[\mu \mathrm{V}]$} & {$[\mu \mathrm{V} . \mathrm{Min}]$} & {$[\%]$} \\
\hline 1 & UNKNOWN & 5.01 & 5.21 & 5.41 & 0.00 & 49.95 & 599.1 & 80.3 & 49.946 \\
\hline 2 & UNKNOWN & 5.41 & 5.55 & 5.88 & 0.00 & 50.05 & 555.9 & 80.5 & 50.054 \\
\hline & & & & & & & & & \\
\hline Total & & & & & & 100.00 & 1155.0 & 160.8 & 100.000 \\
\hline
\end{tabular}

Enantioenriched 13, 94\% ee

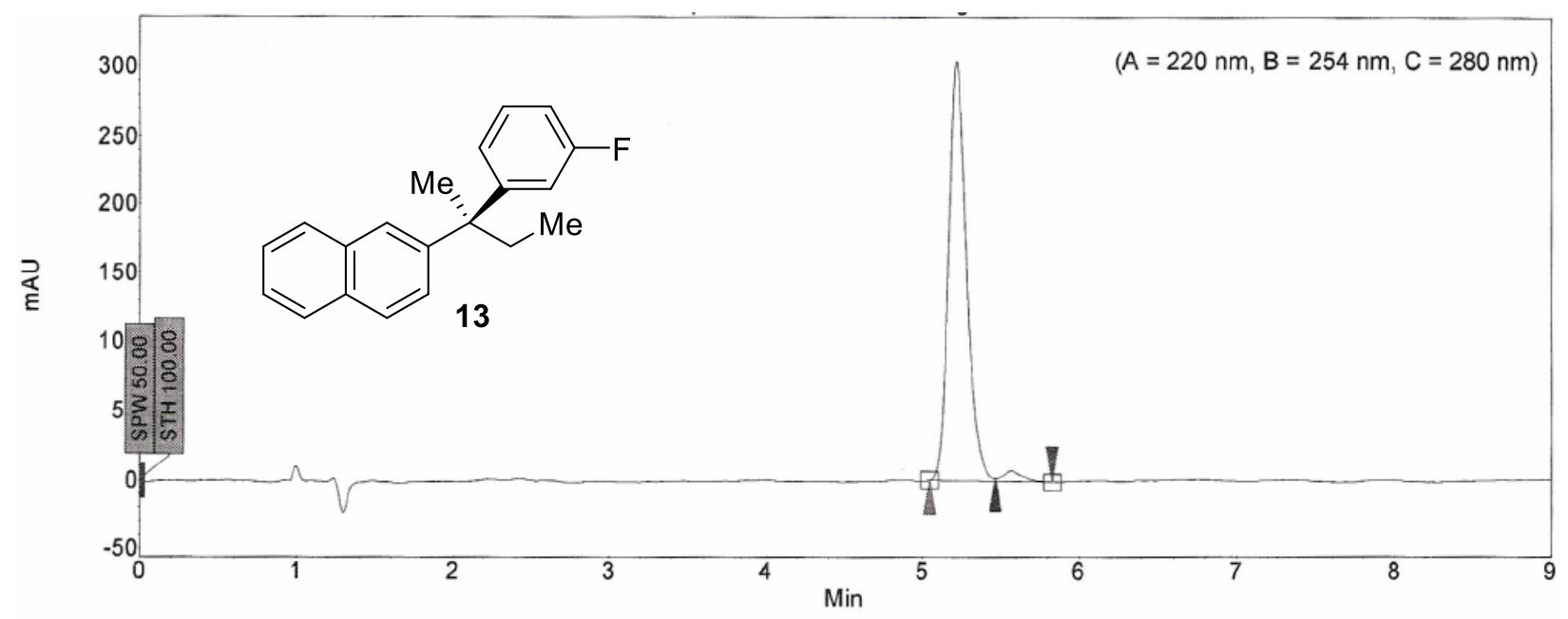

\begin{tabular}{|c|l|r|r|r|r|r|r|r|r|}
\hline Index & Name & Start & Time & End & RT Offset & Quantity & Height & Area & Area \\
\hline \hline & & {$[\mathrm{Min}]$} & {$[\mathrm{Min}]$} & {$[\mathrm{Min}]$} & {$[\mathrm{Min}]$} & {$[\%$ Area] } & {$[\mu \mathrm{V}]$} & {$[\mu \mathrm{V} . \mathrm{Min}]$} & {$[\%]$} \\
\hline 1 & UNKNOWN & 5.05 & 5.22 & 5.47 & 0.00 & 97.13 & 303.5 & 40.2 & 97.132 \\
\hline 2 & UNKNOWN & 5.47 & 5.57 & 5.83 & 0.00 & 2.87 & 7.8 & 1.2 & 2.868 \\
\hline & & & & & & & & & \\
\hline Total & & & & & & 100.00 & 311.3 & 41.4 & 100.000 \\
\hline
\end{tabular}


Racemic 14

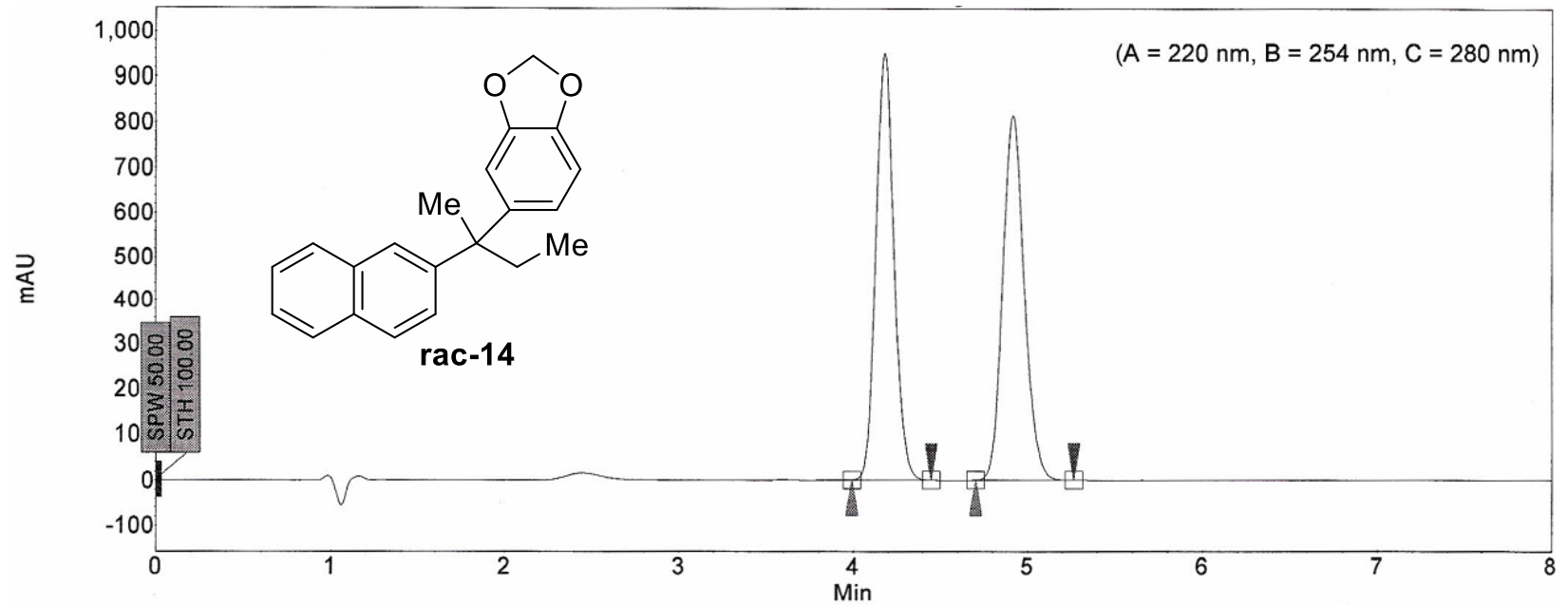

\begin{tabular}{|c|l|r|r|r|r|r|r|r|r|}
\hline Index & Name & Start & Time & End & RT Offset & Quantity & Height & Area & Area \\
\hline \hline & & {$[$ Min] } & {$[$ Min] } & {$[$ Min] } & {$[$ Min] } & {$[\%$ Area] } & {$[\mu \mathrm{V}]$} & {$[\mu \mathrm{V}$. Min] } & {$[\%]$} \\
\hline 1 & UNKNOWN & 3.99 & 4.18 & 4.45 & 0.00 & 49.67 & 950.2 & 114.9 & 49.670 \\
\hline 2 & UNKNOWN & 4.70 & 4.91 & 5.27 & 0.00 & 50.33 & 814.4 & 116.4 & 50.330 \\
\hline & & & & & & & & & \\
\hline Total & & & & & & 100.00 & 1764.6 & 231.2 & 100.000 \\
\hline
\end{tabular}

Enantioenriched 14, 92\% ee

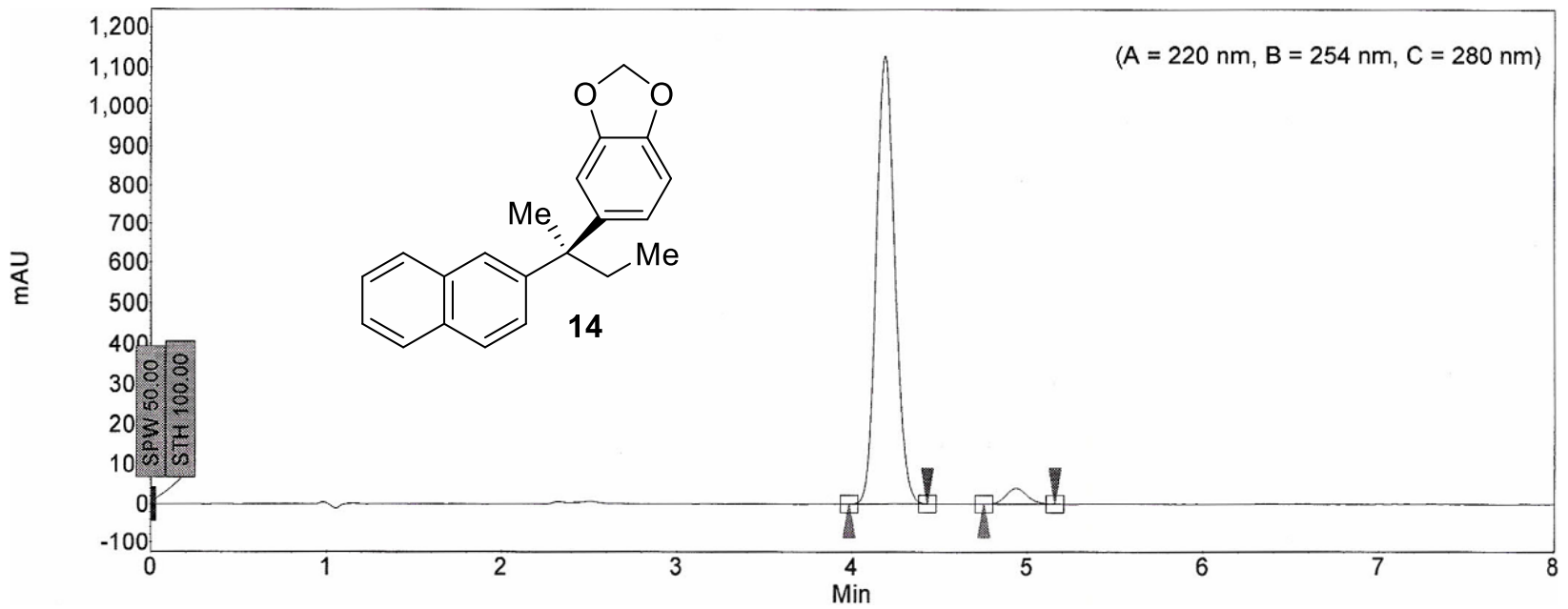

\begin{tabular}{|c|l|r|r|r|r|r|r|r|r|}
\hline Index & Name & Start & Time & End & RT Offset & Quantity & Height & Area & Area \\
\hline \hline & & {$[\mathrm{Min}]$} & {$[\mathrm{Min}]$} & {$[\mathrm{Min}]$} & {$[\mathrm{Min}]$} & {$[\%$ Area] } & {$[\mu \mathrm{V}]$} & {$[\mu \mathrm{V} . \mathrm{Min}]$} & {$[\%]$} \\
\hline 1 & UNKNOWN & 3.98 & 4.19 & 4.43 & 0.00 & 96.07 & 1130.0 & 139.5 & 96.066 \\
\hline 2 & UNKNOWN & 4.75 & 4.93 & 5.16 & 0.00 & 3.93 & 41.1 & 5.7 & 3.934 \\
\hline & & & & & & & & & \\
\hline Total & & & & & & 100.00 & 1171.2 & 145.2 & 100.000 \\
\hline
\end{tabular}


Racemic 15

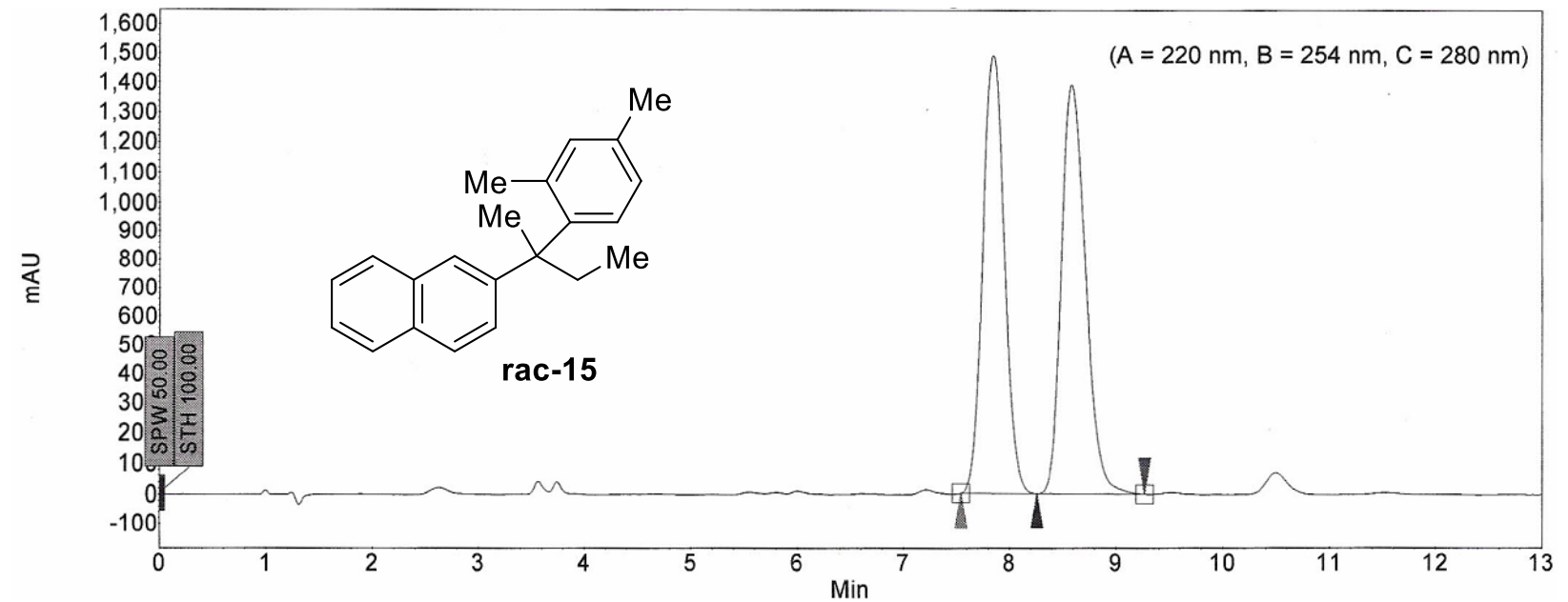

\begin{tabular}{|c|l|r|r|r|r|r|r|r|r|}
\hline Index & Name & Start & Time & End & RT Offset & Quantity & Height & Area & Area \\
\hline & & {$[$ Min] } & {$[$ Min] } & {$[$ Min] } & {$[$ Min] } & {$[\%$ Area] } & {$[\mu \mathrm{V}]$} & {$[\mu \mathrm{V}$. Min] } & {$[\%]$} \\
\hline 1 & UNKNOWN & 7.55 & 7.85 & 8.26 & 0.00 & 49.14 & 1491.2 & 352.2 & 49.145 \\
\hline 2 & UNKNOWN & 8.26 & 8.59 & 9.27 & 0.00 & 50.86 & 1391.4 & 364.5 & 50.855 \\
\hline & & & & & & & & & \\
\hline Total & & & & & & 100.00 & 2882.6 & 716.8 & 100.000 \\
\hline
\end{tabular}

Enantioenriched 15, 95\% ee

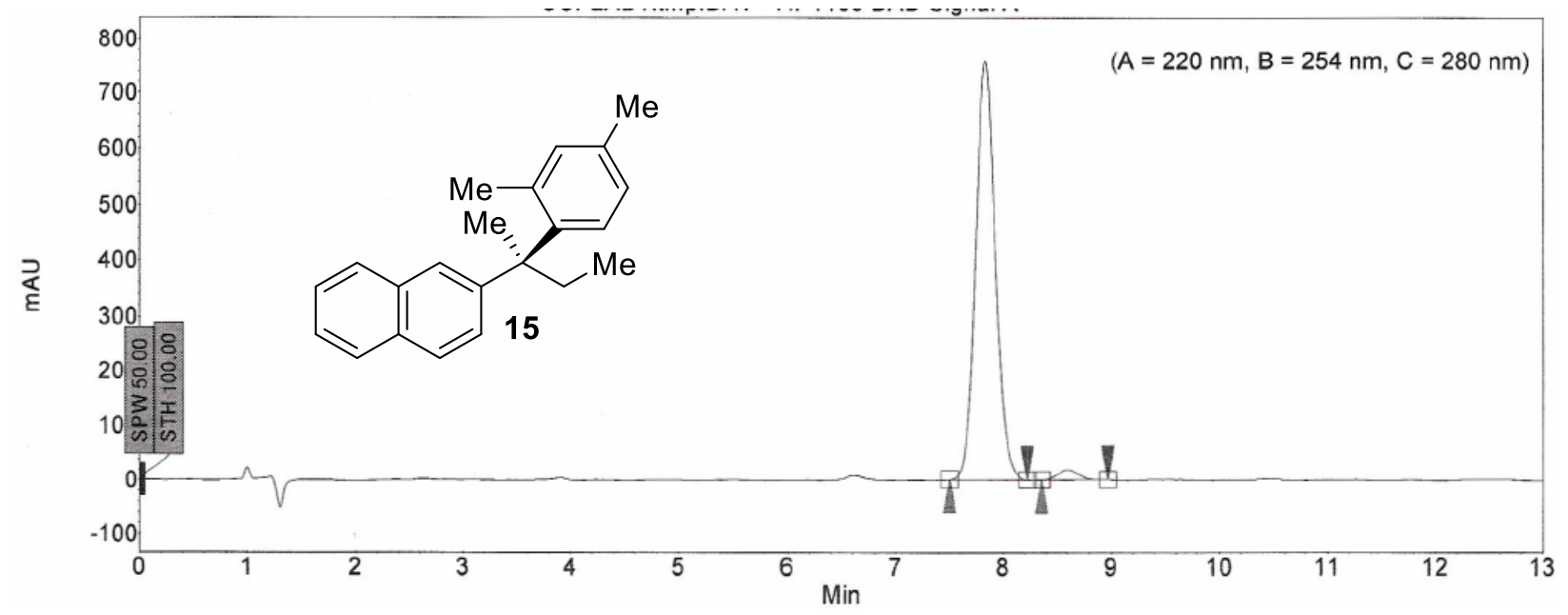

\begin{tabular}{|c|l|r|r|r|r|r|r|r|r|}
\hline Index & Name & Start & Time & End & RT Offset & Quantity & Height & Area & Area \\
\hline \hline & & {$[\mathrm{Min}]$} & {$[\mathrm{Min}]$} & {$[\mathrm{Min}]$} & {$[\mathrm{Min}]$} & {$[\%$ Area] } & {$[\mu \mathrm{V}]$} & {$[\mu \mathrm{V} . \mathrm{Min}]$} & {$[\%]$} \\
\hline 1 & UNKNOWN & 7.50 & 7.83 & 8.22 & 0.00 & 97.52 & 758.0 & 161.1 & 97.517 \\
\hline 2 & UNKNOWN & 8.36 & 8.59 & 8.97 & 0.00 & 2.48 & 18.4 & 4.1 & 2.483 \\
\hline & & & & & & & & & \\
\hline Total & & & & & & 100.00 & 776.4 & 165.2 & 100.000 \\
\hline
\end{tabular}


Racemic 16

mAU

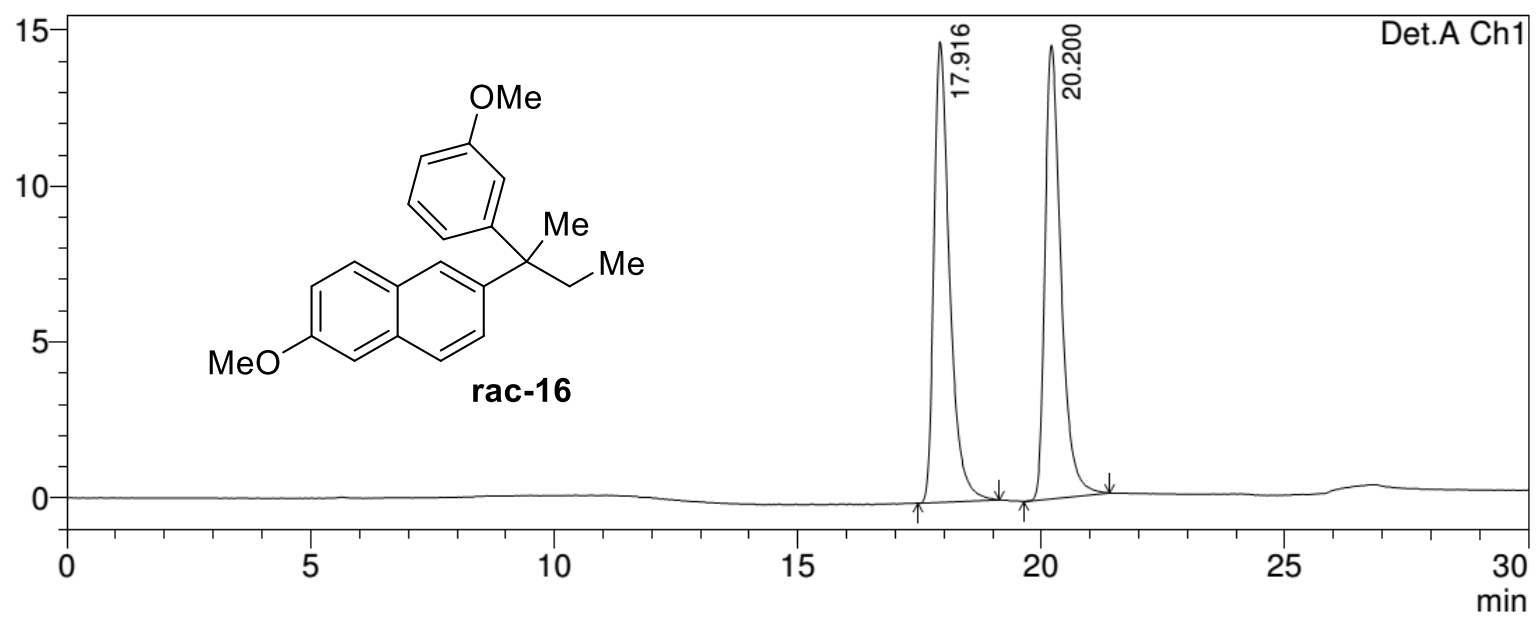

Detector A Ch1 254nm

\begin{tabular}{|r|r|r|r|r|r|}
\hline Peak\# & Ret. Time & \multicolumn{1}{|c|}{ Area } & \multicolumn{1}{|c|}{ Height } & Area \% & \multicolumn{1}{c|}{ Height \% } \\
\hline 1 & 17.916 & 330741 & 14782 & 49.859 & 50.395 \\
\hline 2 & 20.200 & 332617 & 14550 & 50.141 & 49.605 \\
\hline Total & & 663357 & 29332 & 100.000 & 100.000 \\
\hline
\end{tabular}

Enantioenriched 16, 90\% ee

mAU

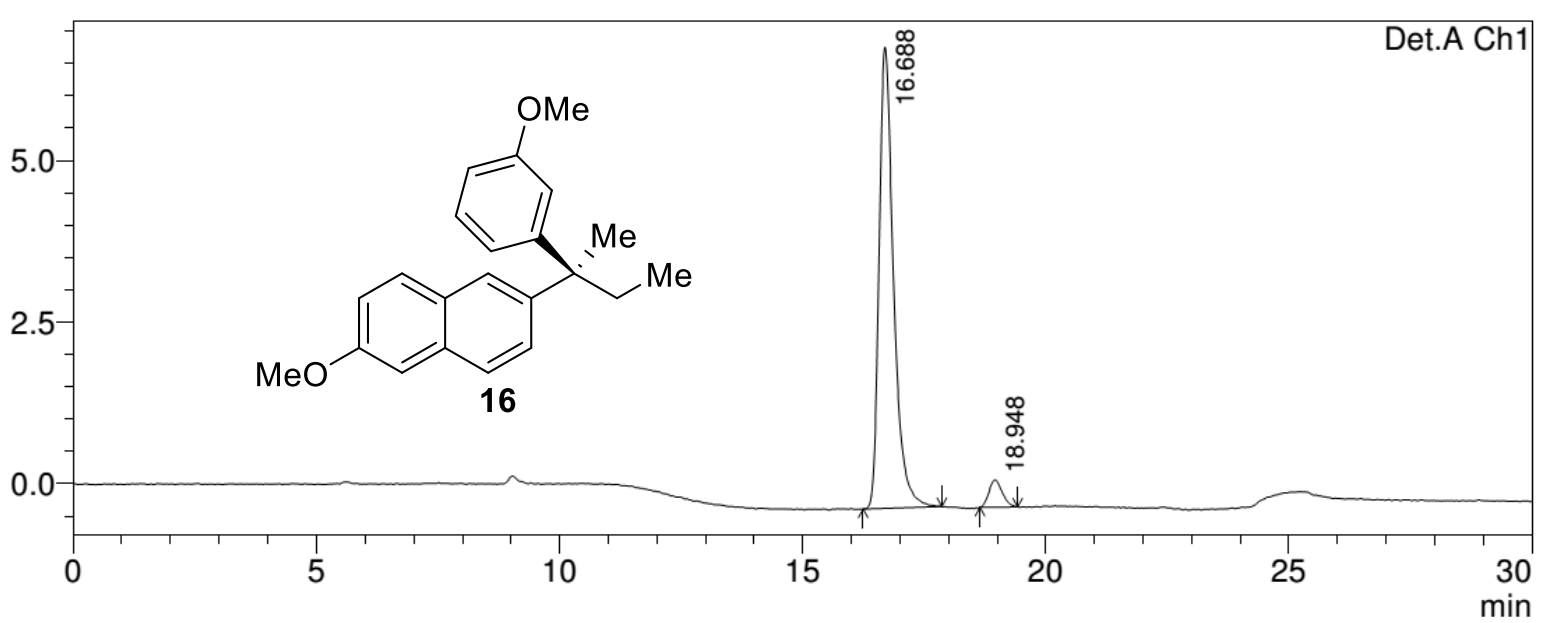

Detector A Ch1 254nm

\begin{tabular}{|r|r|r|r|r|r|}
\hline Peak\# & Ret. Time & \multicolumn{1}{|c|}{ Area } & \multicolumn{1}{|c|}{ Height } & Area \% & \multicolumn{1}{c|}{ Height \% } \\
\hline 1 & 16.688 & 147777 & 7132 & 94.789 & 94.386 \\
\hline 2 & 18.948 & 8124 & 424 & 5.211 & 5.614 \\
\hline Total & & 155901 & 7556 & 100.000 & 100.000 \\
\hline
\end{tabular}


Racemic 17

mAU

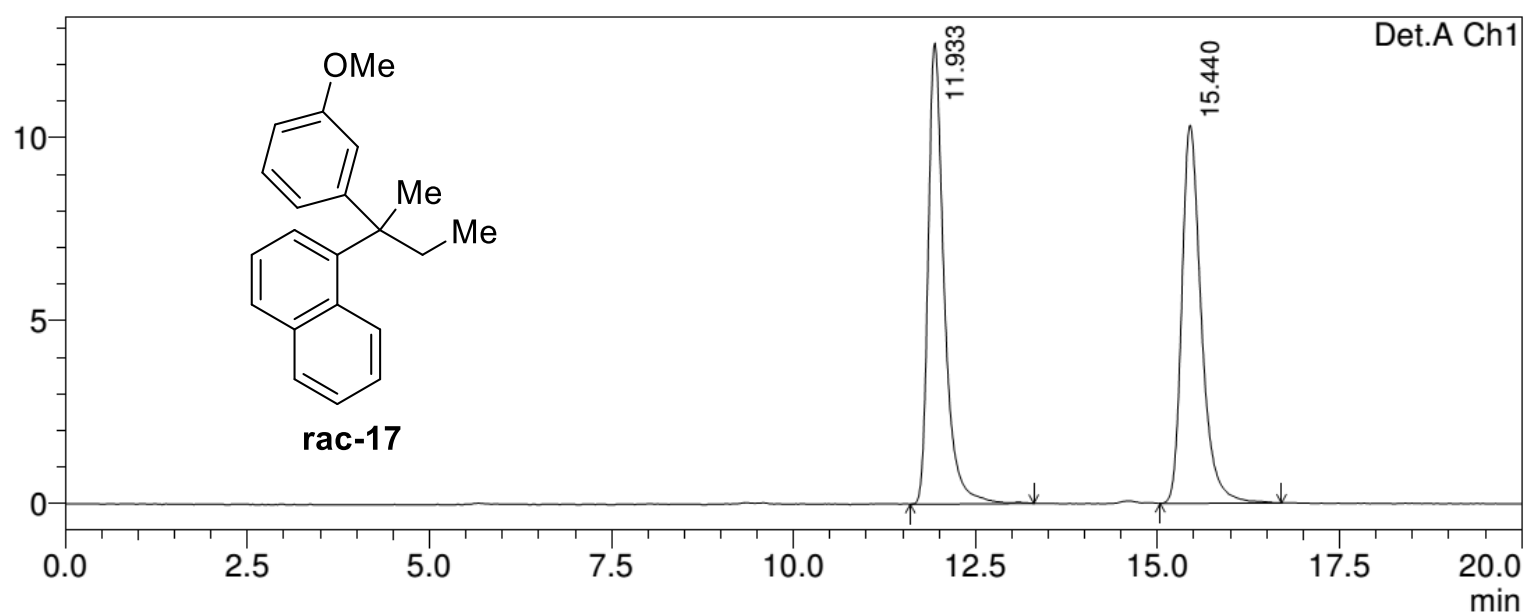

Detector A Ch1 254nm

\begin{tabular}{|r|r|r|r|r|r|}
\hline \multicolumn{1}{|c|}{ Peak\# } & Ret. Time & Area & Height & Area \% & Height \% \\
\hline 1 & 11.933 & 192477 & 12599 & 50.018 & 54.934 \\
\hline 2 & 15.440 & 192342 & 10336 & 49.982 & 45.066 \\
\hline Total & & 384819 & 22934 & 100.000 & 100.000 \\
\hline
\end{tabular}

Enantioenriched 17, 88\% ee

mAU

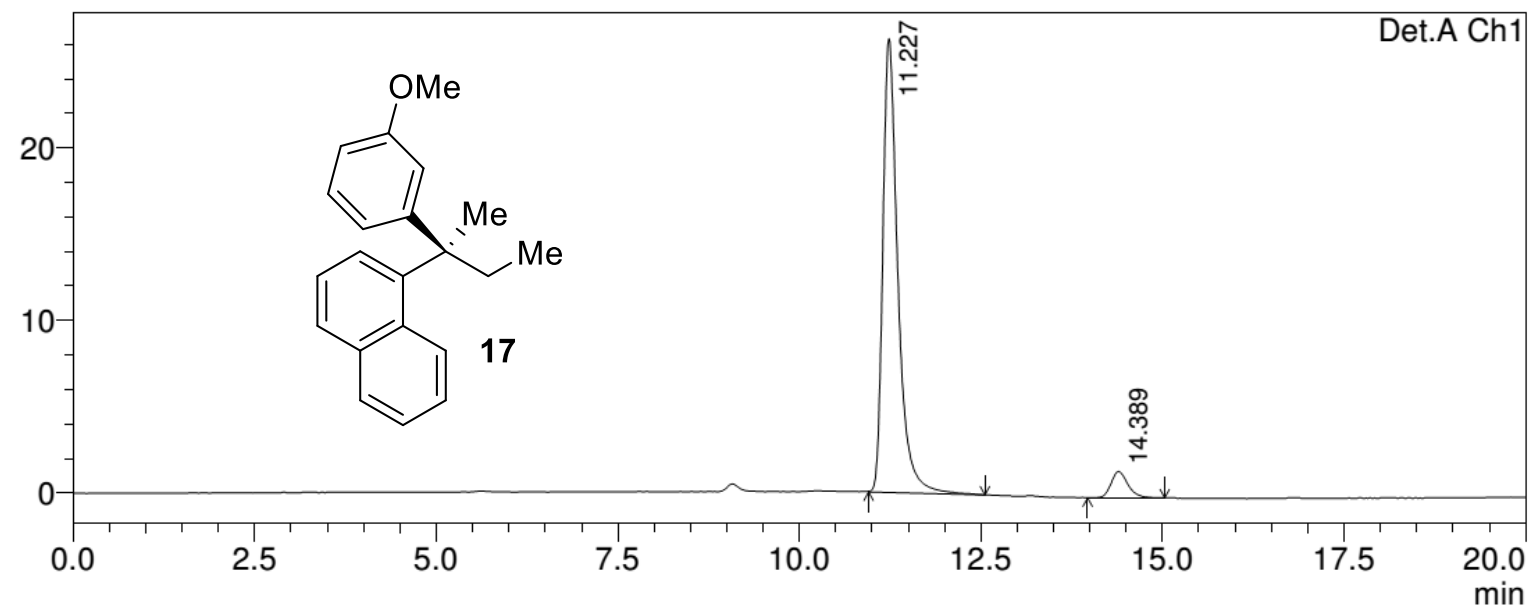

Detector A Ch1 254nm

\begin{tabular}{|r|r|r|r|r|r|}
\hline \multicolumn{1}{|c|}{ Peak\# } & Ret. Time & \multicolumn{1}{c|}{ Area } & Height & Area \% & Height \% \\
\hline 1 & 11.227 & 378067 & 26292 & 93.832 & 94.517 \\
\hline 2 & 14.389 & 24853 & 1525 & 6.168 & 5.483 \\
\hline Total & & 402920 & 27817 & 100.000 & 100.000 \\
\hline
\end{tabular}


Racemic 18

mAU

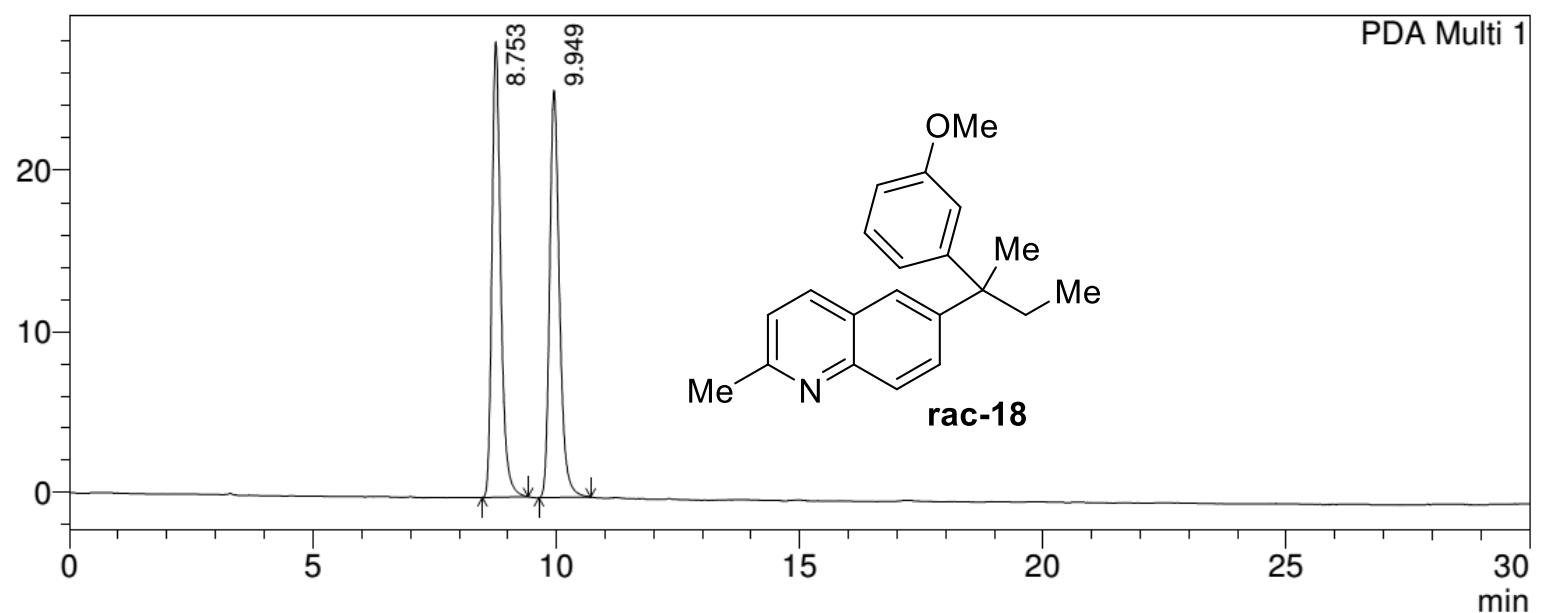

PDA Ch1 254nm 4nm

\begin{tabular}{|r|r|r|r|r|r|}
\hline \multicolumn{1}{|c|}{ Peak\# } & Ret. Time & \multicolumn{1}{c|}{ Area } & Height & Area \% & \multicolumn{1}{c|}{ Height \% } \\
\hline 1 & 8.753 & 355326 & 28276 & 50.038 & 52.795 \\
\hline 2 & 9.949 & 354785 & 25282 & 49.962 & 47.205 \\
\hline Total & & 710111 & 53557 & 100.000 & 100.000 \\
\hline
\end{tabular}

Enantioenriched 18, 97\% ee

mAU

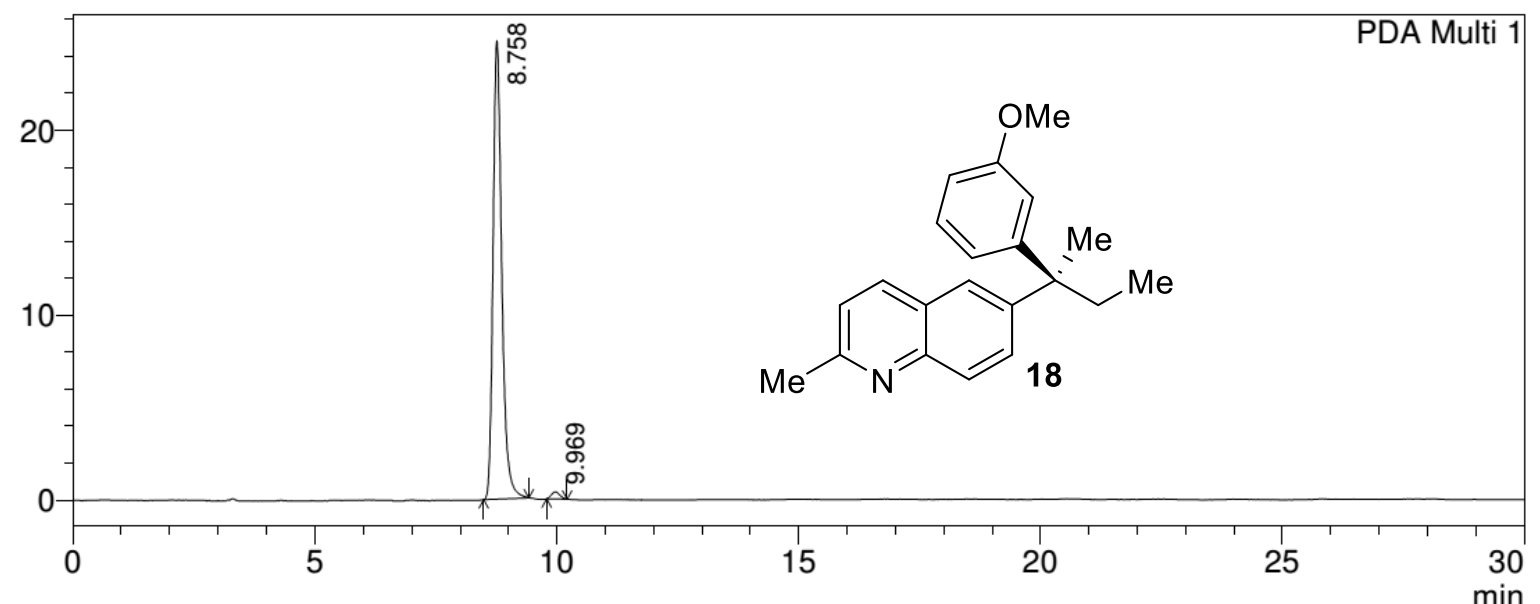

PDA Ch1 254nm 4nm

\begin{tabular}{|r|r|r|r|r|r|}
\hline \multicolumn{1}{|c|}{ Peak\# } & \multicolumn{1}{|c|}{ Ret. Time } & \multicolumn{1}{c|}{ Area } & \multicolumn{1}{c|}{ Height } & Area \% & Height \% \\
\hline 1 & 8.758 & 310956 & 24796 & 98.496 & 98.455 \\
\hline 2 & 9.969 & 4747 & 389 & 1.504 & 1.545 \\
\hline Total & & 315703 & 25185 & 100.000 & 100.000 \\
\hline
\end{tabular}


Racemic 19

mAU

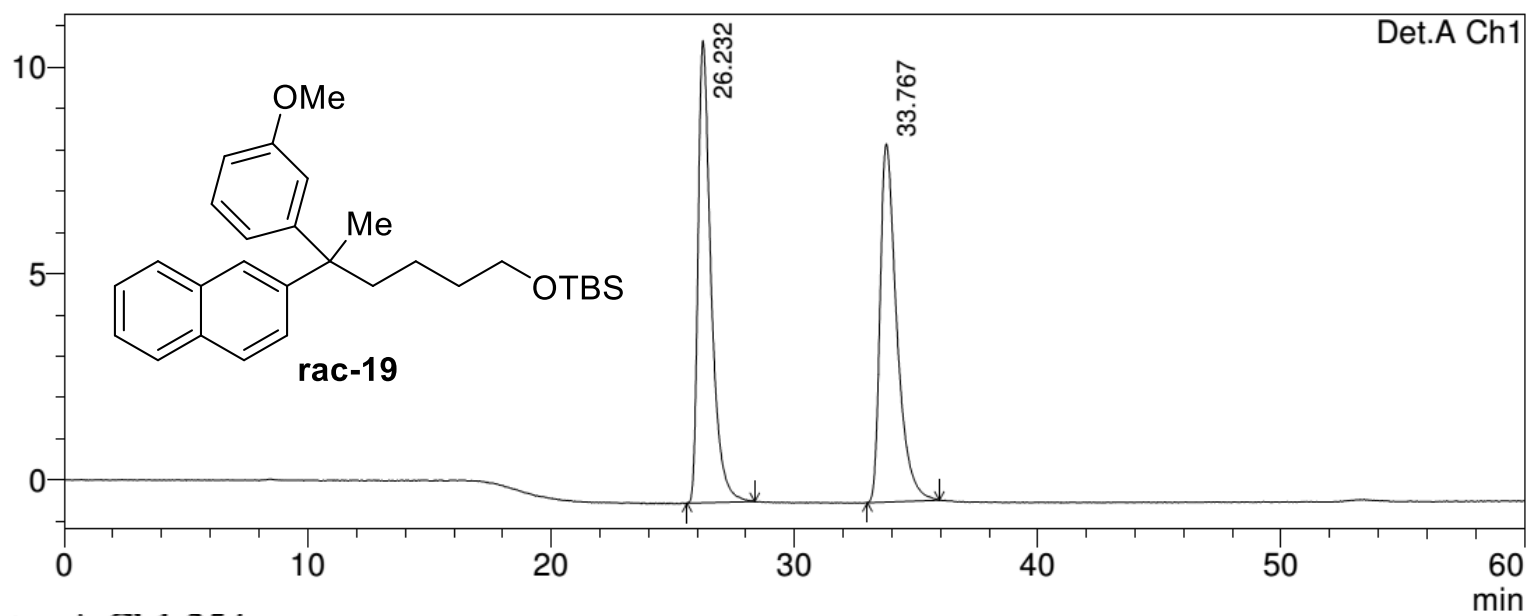

Detector A Ch1 254nm

\begin{tabular}{|r|r|r|r|r|r|}
\hline \multicolumn{1}{|c|}{ Peak\# } & Ret. Time & \multicolumn{1}{c|}{ Area } & Height & Area \% & \multicolumn{1}{c|}{ Height \% } \\
\hline 1 & 26.232 & 416026 & 11202 & 50.139 & 56.329 \\
\hline 2 & 33.767 & 413712 & 8684 & 49.861 & 43.671 \\
\hline Total & & 829738 & 19886 & 100.000 & 100.000 \\
\hline
\end{tabular}

Enantioenriched 19, 99\% ee

mAU

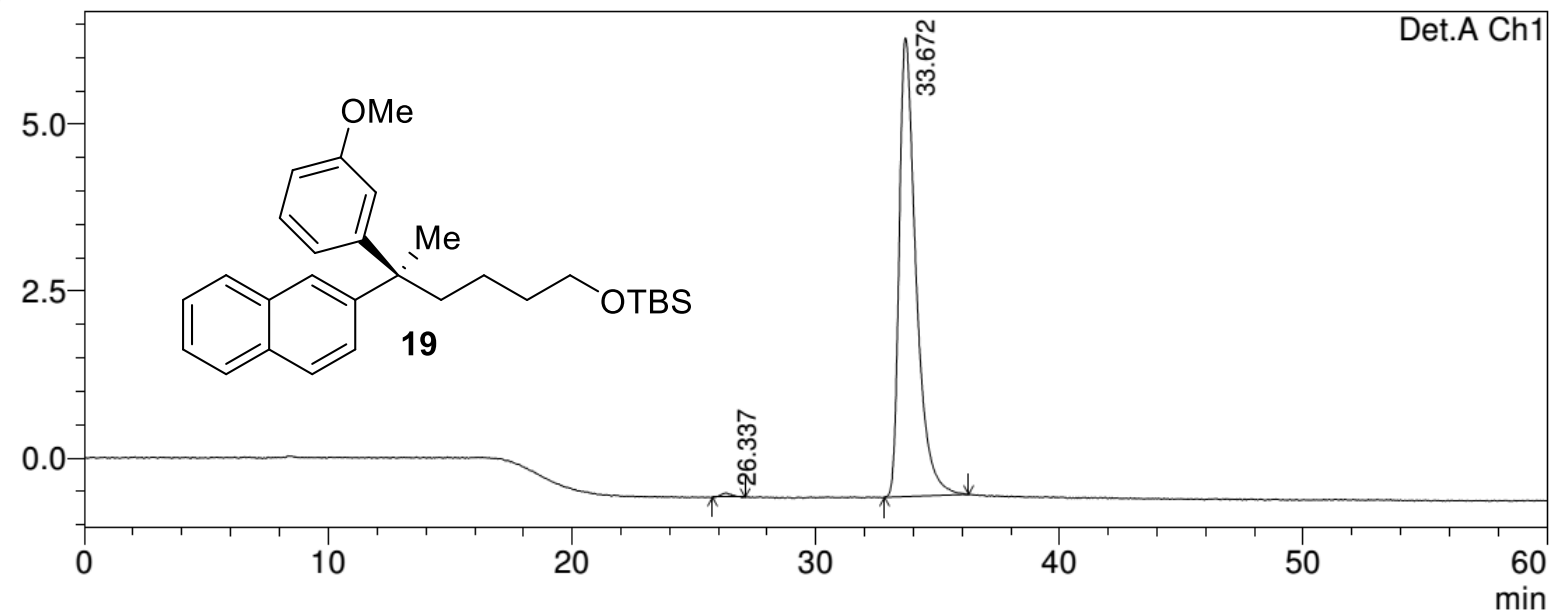

Detector A Ch1 254nm

\begin{tabular}{|r|r|r|r|r|r|}
\hline \multicolumn{1}{|c|}{ Peak\# } & Ret. Time & \multicolumn{1}{c|}{ Area } & \multicolumn{1}{|c|}{ Height } & Area \% & \multicolumn{1}{c|}{ Height \% } \\
\hline 1 & 26.337 & 2073 & 64 & 0.625 & 0.925 \\
\hline 2 & 33.672 & 329423 & 6878 & 99.375 & 99.075 \\
\hline Total & & 331496 & 6942 & 100.000 & 100.000 \\
\hline
\end{tabular}


Racemic $\mathbf{2 0}$

mAU

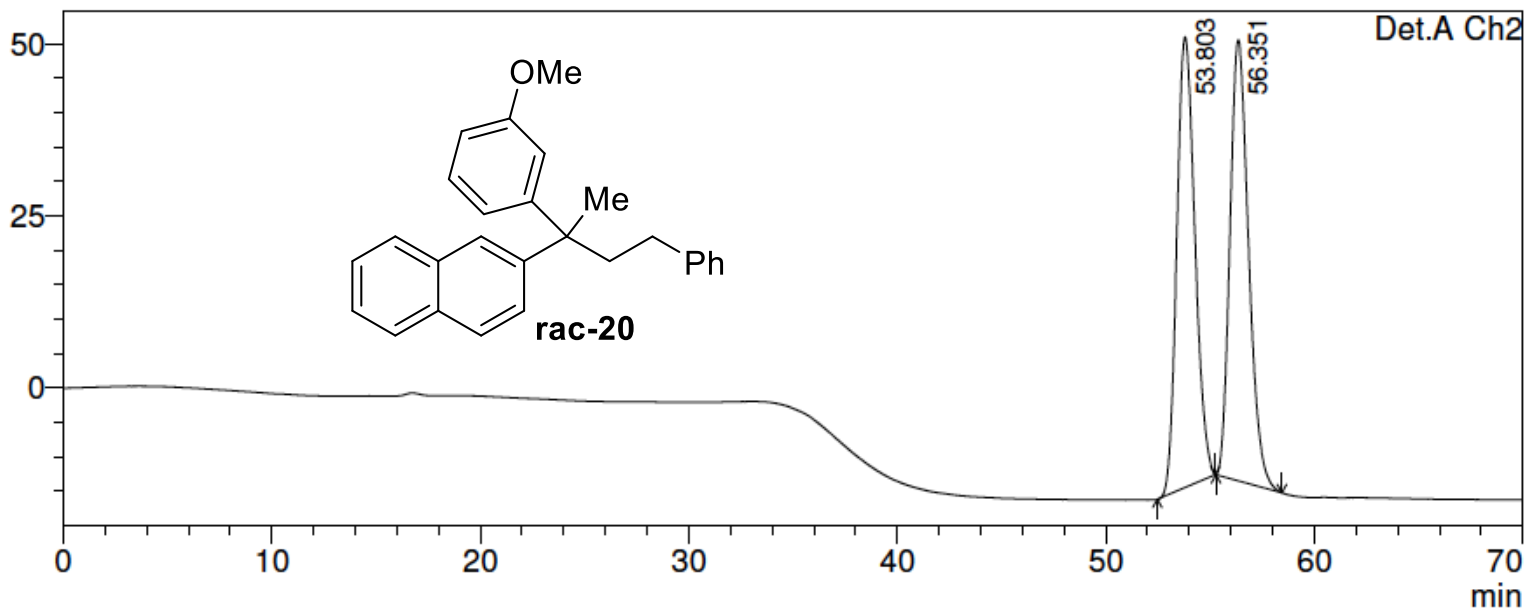

Detector A Ch2 220nm

\begin{tabular}{|r|r|r|r|r|r|}
\hline Peak\# & \multicolumn{1}{|c|}{ Ret. Time } & \multicolumn{1}{|c|}{ Area } & Height & \multicolumn{1}{|c|}{ Area \% } & Height \% \\
\hline 1 & 53.803 & 3938543 & 65377 & 49.786 & 50.537 \\
\hline 2 & 56.351 & 3972469 & 63987 & 50.214 & 49.463 \\
\hline Total & & 7911011 & 129364 & 100.000 & 100.000 \\
\hline
\end{tabular}

Enantioenriched 20, 94\% ee

mAU

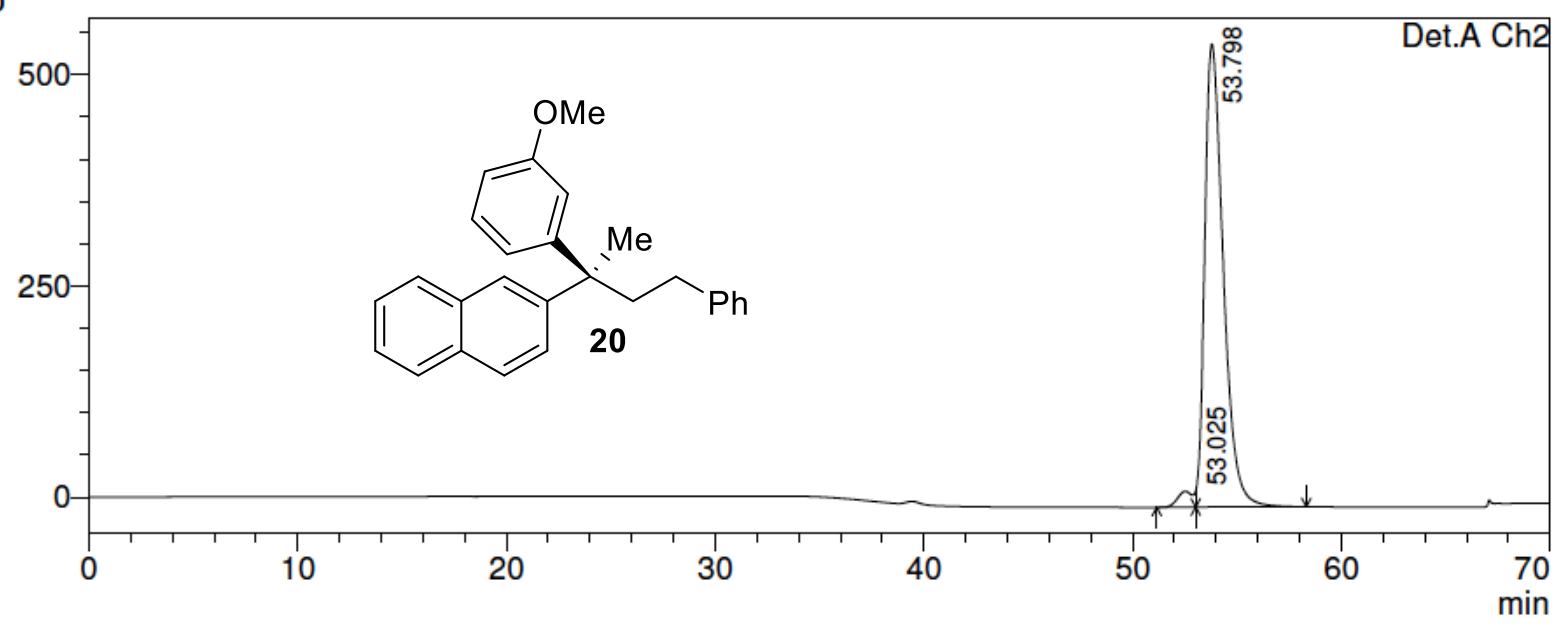

Detector A Ch2 220nm

\begin{tabular}{|r|r|r|r|r|r|}
\hline Peak\# & \multicolumn{1}{|c|}{ Ret. Time } & \multicolumn{1}{c|}{ Area } & \multicolumn{1}{|c|}{ Height } & \multicolumn{1}{c|}{ Area \% } & \multicolumn{1}{c|}{ Height \% } \\
\hline 1 & 53.025 & 977426 & 19027 & 2.811 & 3.355 \\
\hline 2 & 53.798 & 33790474 & 548101 & 97.189 & 96.645 \\
\hline Total & & 34767901 & 567128 & 100.000 & 100.000 \\
\hline
\end{tabular}


Racemic 21

mAU

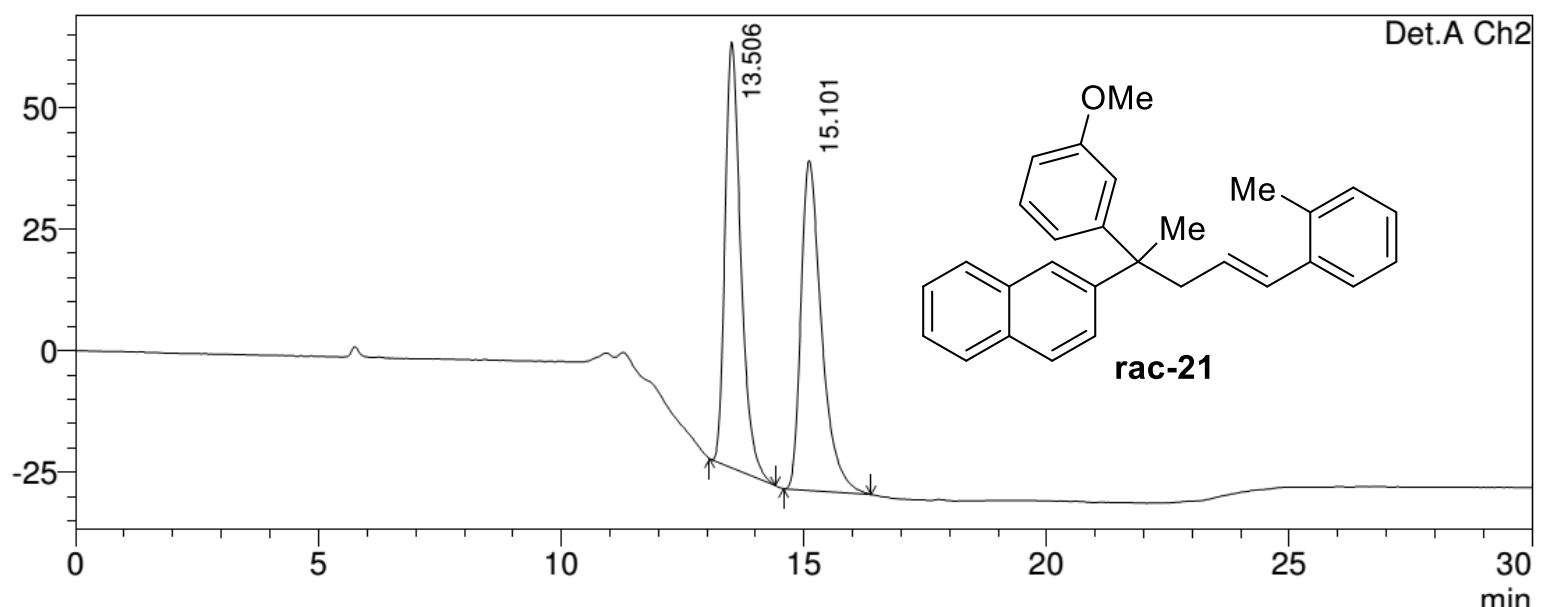

\begin{tabular}{|r|r|r|r|r|r|}
\hline \multicolumn{1}{|c|}{ Peak\# } & Ret. Time & Area & Height & Area \% & Height \% \\
\hline 1 & 13.506 & 1981293 & 87791 & 49.645 & 56.359 \\
\hline 2 & 15.101 & 2009656 & 67980 & 50.355 & 43.641 \\
\hline Total & & 3990949 & 155771 & 100.000 & 100.000 \\
\hline
\end{tabular}

Enantioenriched 21, 94\% ee

$\mathrm{mAU}$

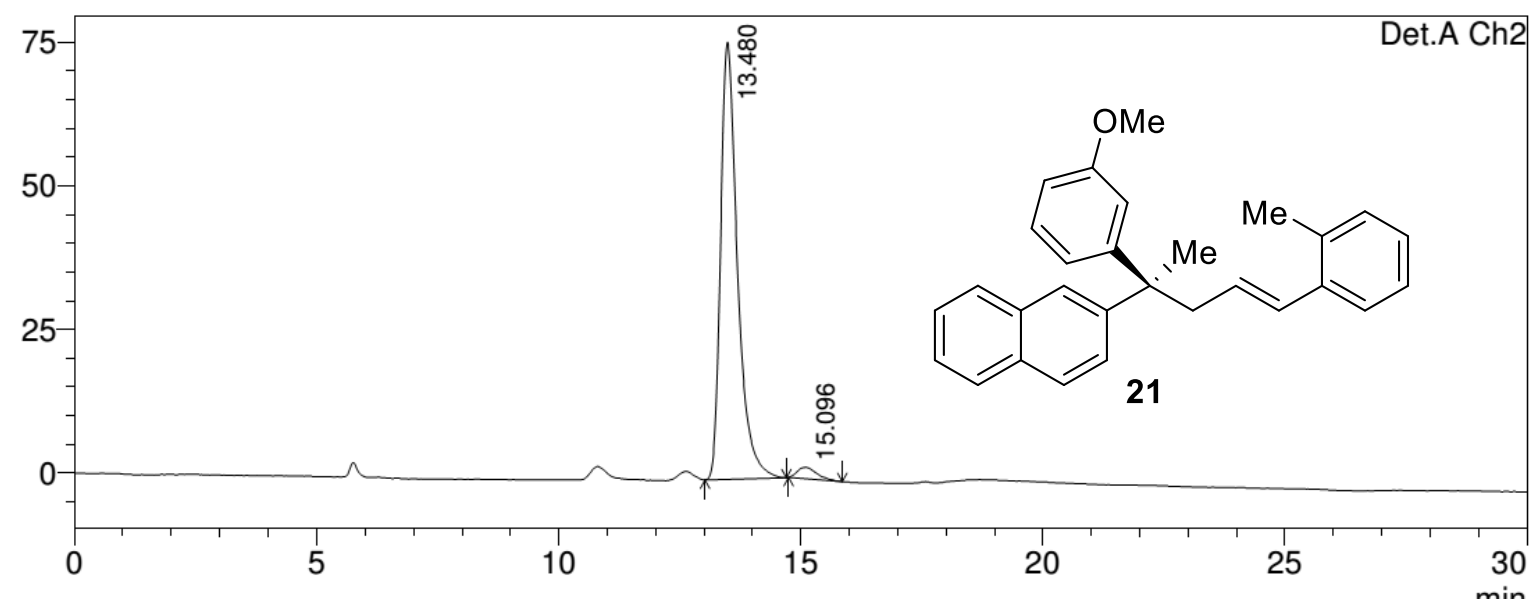

\begin{tabular}{|r|r|r|r|r|r|}
\hline \multicolumn{1}{|c|}{ Peak\# } & Ret. Time & \multicolumn{1}{c|}{ Area } & Height & Area \% & \multicolumn{1}{c|}{ Height \% } \\
\hline 1 & 13.480 & 1845100 & 76078 & 97.047 & 97.444 \\
\hline 2 & 15.096 & 56141 & 1996 & 2.953 & 2.556 \\
\hline Total & & 1901241 & 78073 & 100.000 & 100.000 \\
\hline
\end{tabular}


Racemic 22

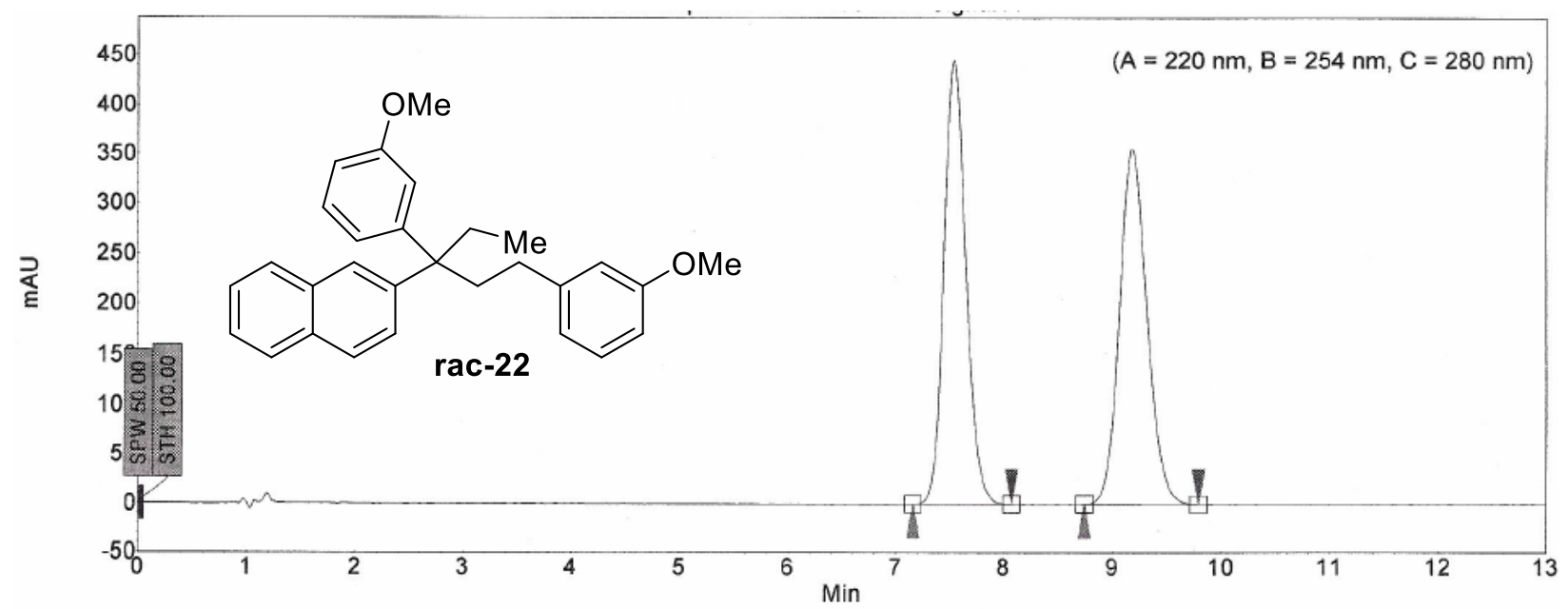

\begin{tabular}{|c|l|r|r|r|r|r|r|r|r|}
\hline Index & Name & Start & Time & End & RT Offset & Quantity & Height & Area & Area \\
\hline \hline & & {$[\mathrm{Min}]$} & {$[\mathrm{Min}]$} & {$[\mathrm{Min}]$} & {$[\mathrm{Min}]$} & {$[\%$ Area] } & {$[\mu \mathrm{V}]$} & {$[\mu \mathrm{V} . \mathrm{Min}]$} & {$[\%]$} \\
\hline 1 & UNKNOWN & 7.16 & 7.54 & 8.07 & 0.00 & 49.93 & 445.3 & 104.9 & 49.926 \\
\hline 2 & UNKNOWN & 8.74 & 9.18 & 9.79 & 0.00 & 50.07 & 356.0 & 105.2 & 50.074 \\
\hline & & & & & & & & & \\
\hline Total & & & & & & 100.00 & 801.3 & 210.1 & 100.000 \\
\hline
\end{tabular}

Enantioenriched 22, 87\% ee

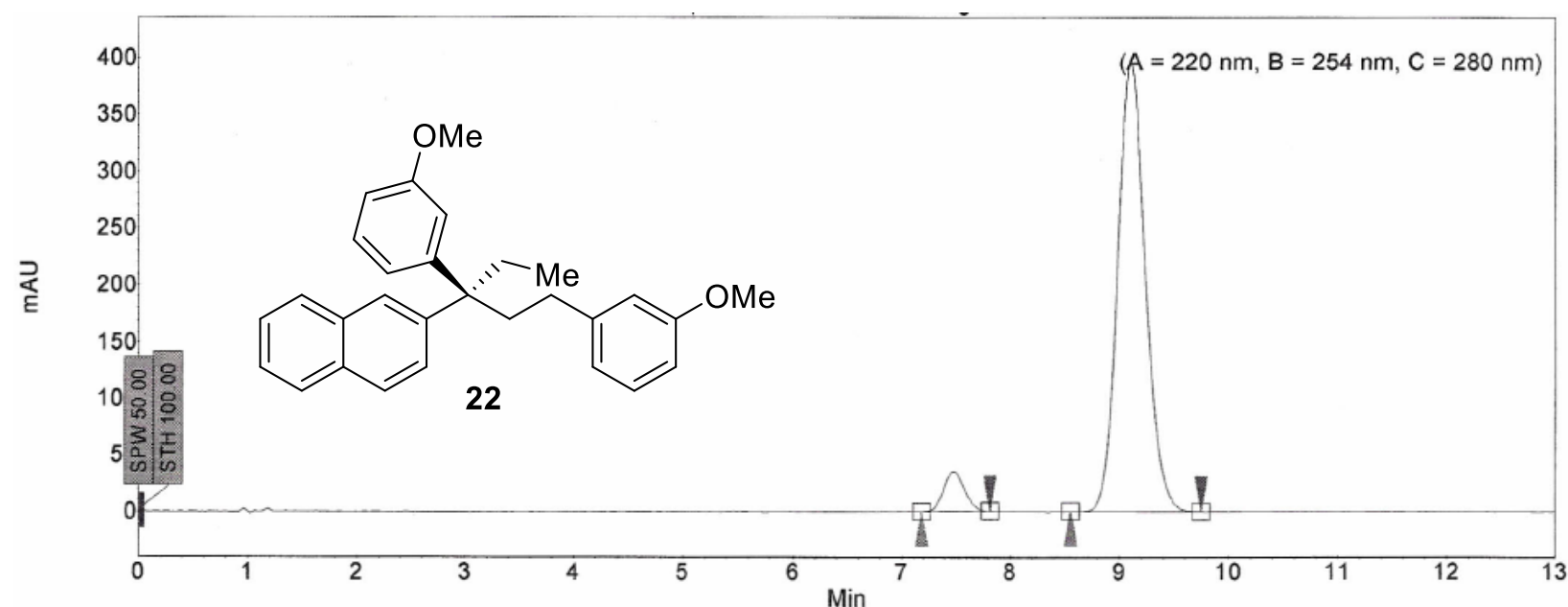

\begin{tabular}{|c|l|r|r|r|r|r|r|r|r|}
\hline Index & Name & Start & Time & End & RT Offset & Quantity & Height & Area & Area \\
\hline \hline & & {$[\mathrm{Min}]$} & {$[\mathrm{Min}]$} & {$[\mathrm{Min}]$} & {$[\mathrm{Min}]$} & {$[\%$ Area] } & {$[\mu \mathrm{V}]$} & {$[\mu \mathrm{V} . \mathrm{Min}]$} & {$[\%]$} \\
\hline 1 & UNKNOWN & 7.18 & 7.48 & 7.81 & 0.00 & 6.28 & 34.8 & 7.8 & 6.282 \\
\hline 2 & UNKNOWN & 8.55 & 9.10 & 9.75 & 0.00 & 93.72 & 396.5 & 116.7 & 93.718 \\
\hline & & & & & & & & & \\
\hline Total & & & & & & 100.00 & 431.3 & 124.6 & 100.000 \\
\hline
\end{tabular}


Racemic 23

$\mathrm{mAU}$

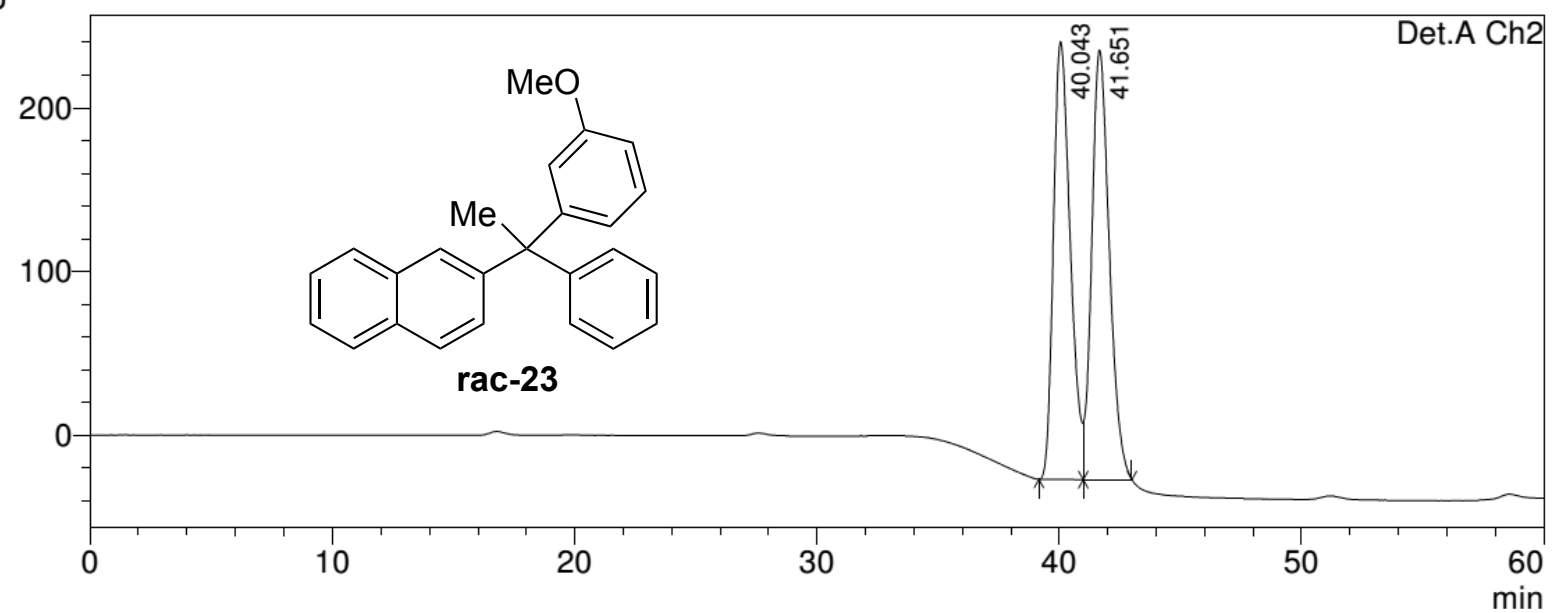

Detector A Ch1 254nm

\begin{tabular}{|r|r|r|r|r|r|}
\hline \multicolumn{1}{|c|}{ Peak\# } & Ret. Time & Area & Height & Area \% & \multicolumn{1}{c|}{ Height \% } \\
\hline 1 & 40.045 & 1348999 & 27748 & 49.573 & 50.539 \\
\hline 2 & 41.650 & 1372253 & 27157 & 50.427 & 49.461 \\
\hline Total & & 2721252 & 54905 & 100.000 & 100.000 \\
\hline
\end{tabular}

Enantioenriched 23, 94\% ee

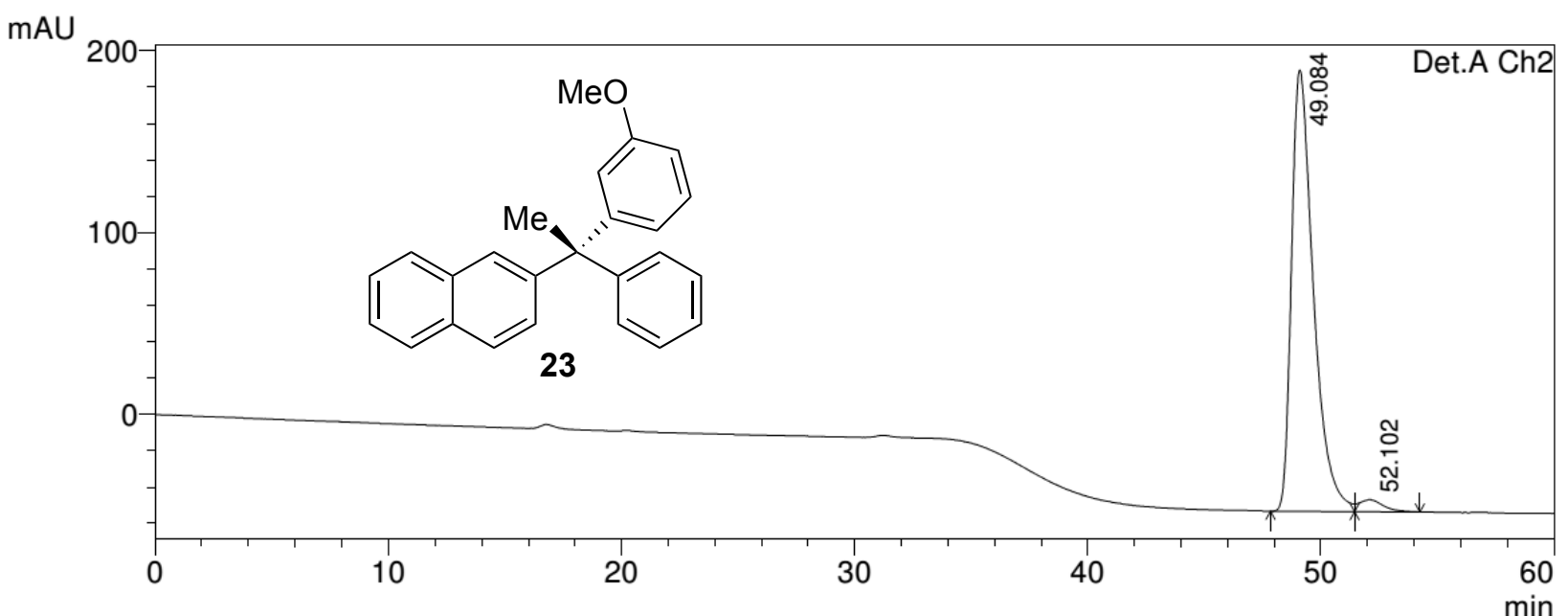

Detector A Ch2 210nm

\begin{tabular}{|r|r|r|r|r|r|}
\hline \multicolumn{1}{|c|}{ Peak\# } & Ret. Time & \multicolumn{1}{c|}{ Area } & \multicolumn{1}{c|}{ Height } & Area \% & Height \% \\
\hline 1 & 49.084 & 15876695 & 242733 & 97.073 & 97.378 \\
\hline 2 & 52.102 & 478750 & 6535 & 2.927 & 2.622 \\
\hline Total & & 16355444 & 249267 & 100.000 & 100.000 \\
\hline
\end{tabular}


Co-injection of racemic $\mathbf{2 3}$ and enantioenriched $\mathbf{2 3}$

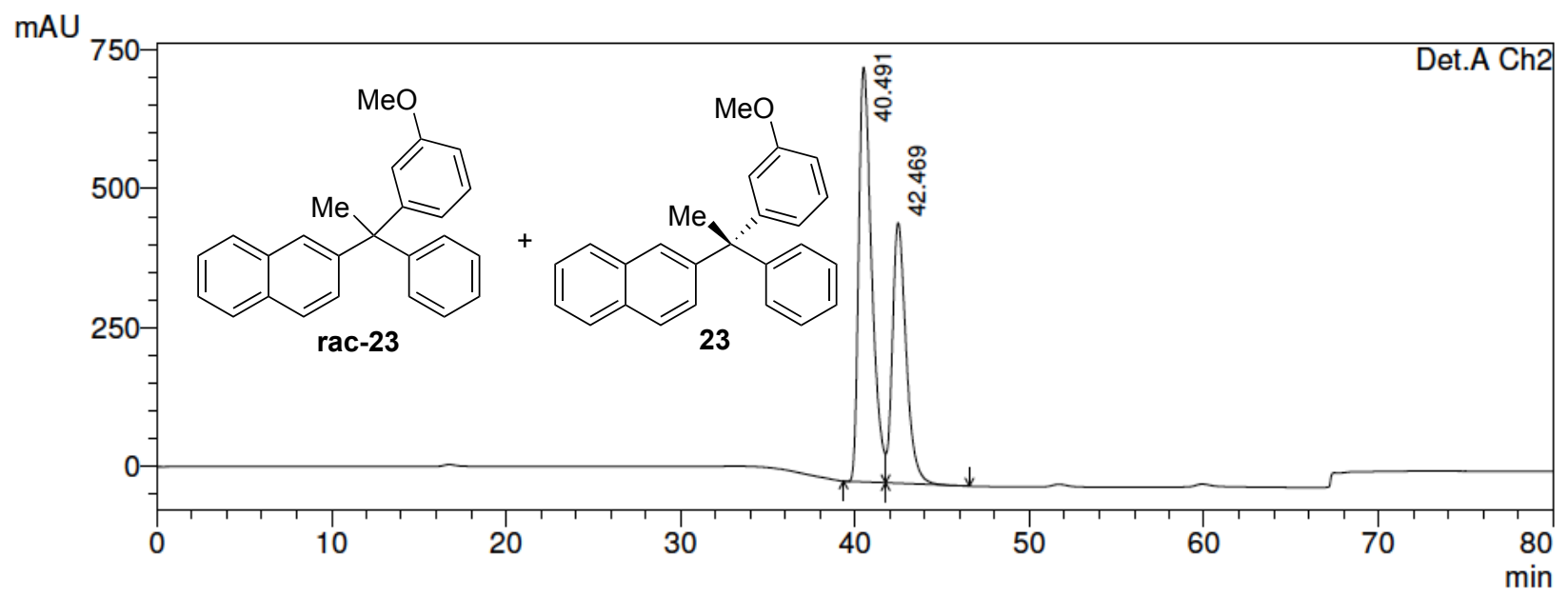

Detector A Ch2 210nm

\begin{tabular}{|r|r|r|r|r|r|}
\hline \multicolumn{1}{|c|}{ Peak\# } & Ret. Time & \multicolumn{1}{c|}{ Area } & Height & Area \% & Height \% \\
\hline 1 & 40.491 & 38652729 & 746411 & 60.136 & 61.431 \\
\hline 2 & 42.469 & 25623023 & 468626 & 39.864 & 38.569 \\
\hline Total & & 64275752 & 1215037 & 100.000 & 100.000 \\
\hline
\end{tabular}


Racemic 24

mAU

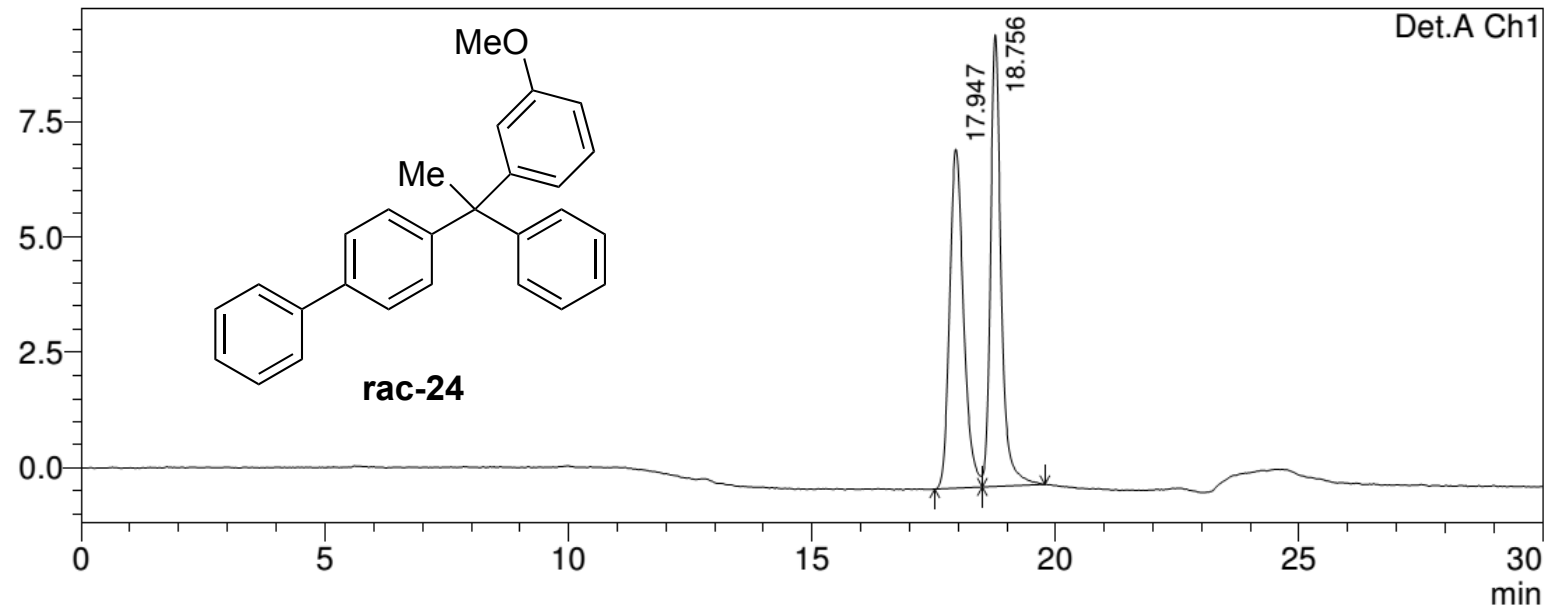

Detector A Ch1 254nm

\begin{tabular}{|r|r|r|r|r|r|}
\hline \multicolumn{1}{|c|}{ Peak\# } & Ret. Time & Area & Height & Area \% & \multicolumn{1}{c|}{ Height \% } \\
\hline 1 & 17.947 & 146239 & 7340 & 49.779 & 42.872 \\
\hline 2 & 18.756 & 147539 & 9781 & 50.221 & 57.128 \\
\hline Total & & 293778 & 17120 & 100.000 & 100.000 \\
\hline
\end{tabular}

Enantioenriched 24, 91\% ee

mAU

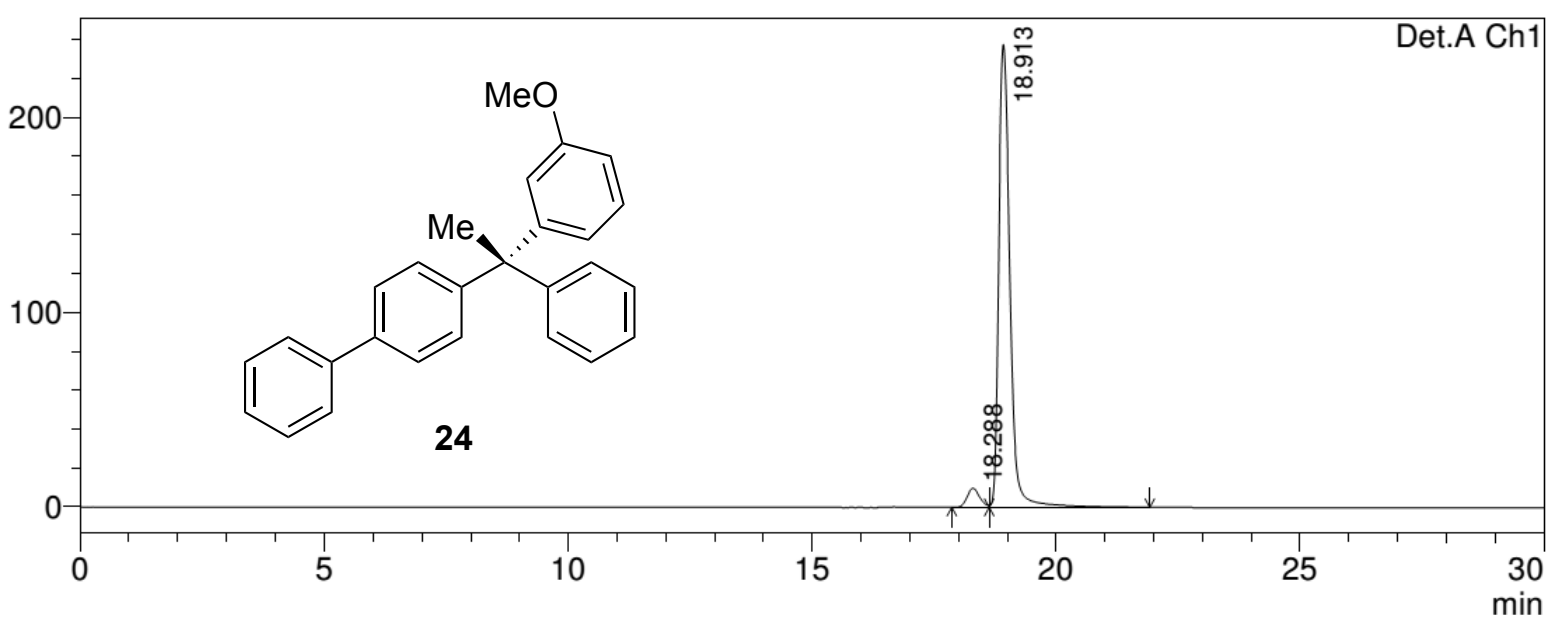

Detector A Ch1 254nm

\begin{tabular}{|r|r|r|r|r|r|}
\hline \multicolumn{1}{|c|}{ Peak\# } & Ret. Time & \multicolumn{1}{c|}{ Area } & \multicolumn{1}{|c|}{ Height } & Area \% & Height \% \\
\hline 1 & 18.288 & 179299 & 9861 & 4.531 & 3.984 \\
\hline 2 & 18.913 & 3777644 & 237657 & 95.469 & 96.016 \\
\hline Total & & 3956943 & 247518 & 100.000 & 100.000 \\
\hline
\end{tabular}


Racemic 1a

mAU

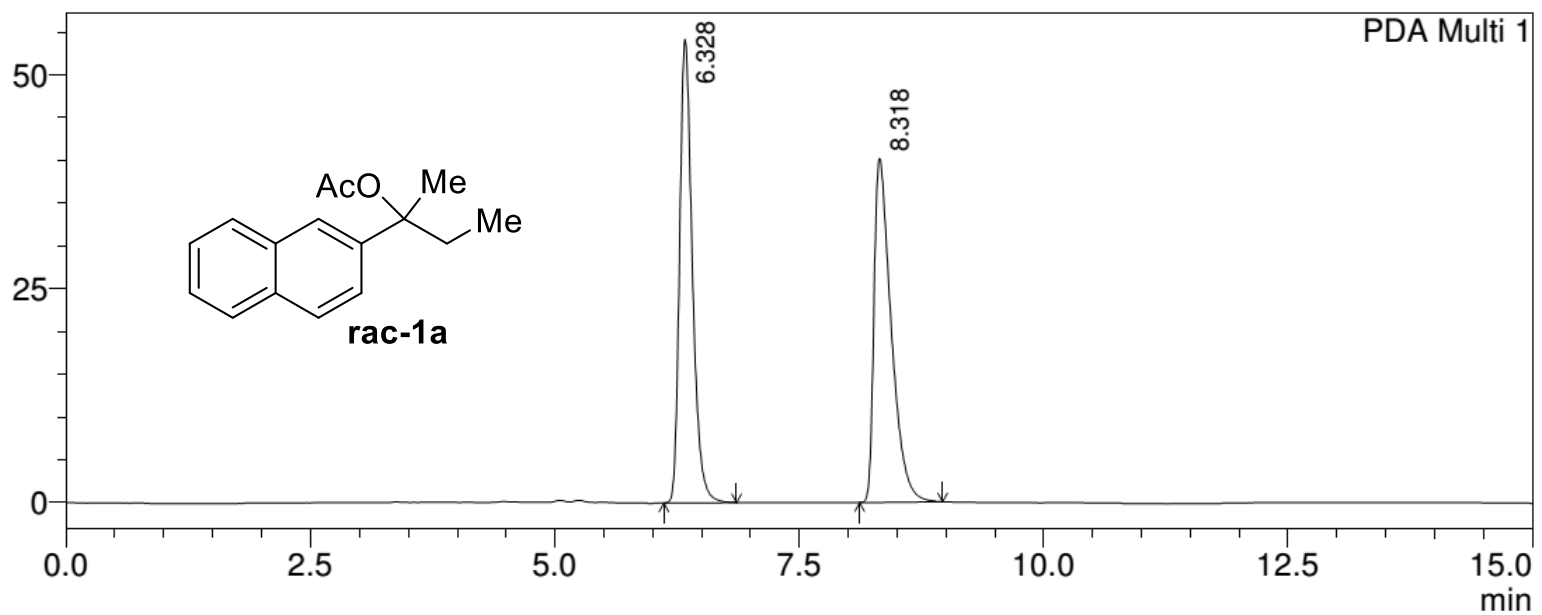

PDA Ch1 254nm 4nm

\begin{tabular}{|r|r|r|r|r|r|}
\hline \multicolumn{1}{|c|}{ Peak\# } & Ret. Time & \multicolumn{1}{|c|}{ Area } & Height & Area \% & \multicolumn{1}{c|}{ Height \% } \\
\hline 1 & 6.328 & 491674 & 54209 & 49.939 & 57.391 \\
\hline 2 & 8.318 & 492882 & 40246 & 50.061 & 42.609 \\
\hline Total & & 984556 & 94456 & 100.000 & 100.000 \\
\hline
\end{tabular}

Enantioenriched 1a, 96\% ee

mAU

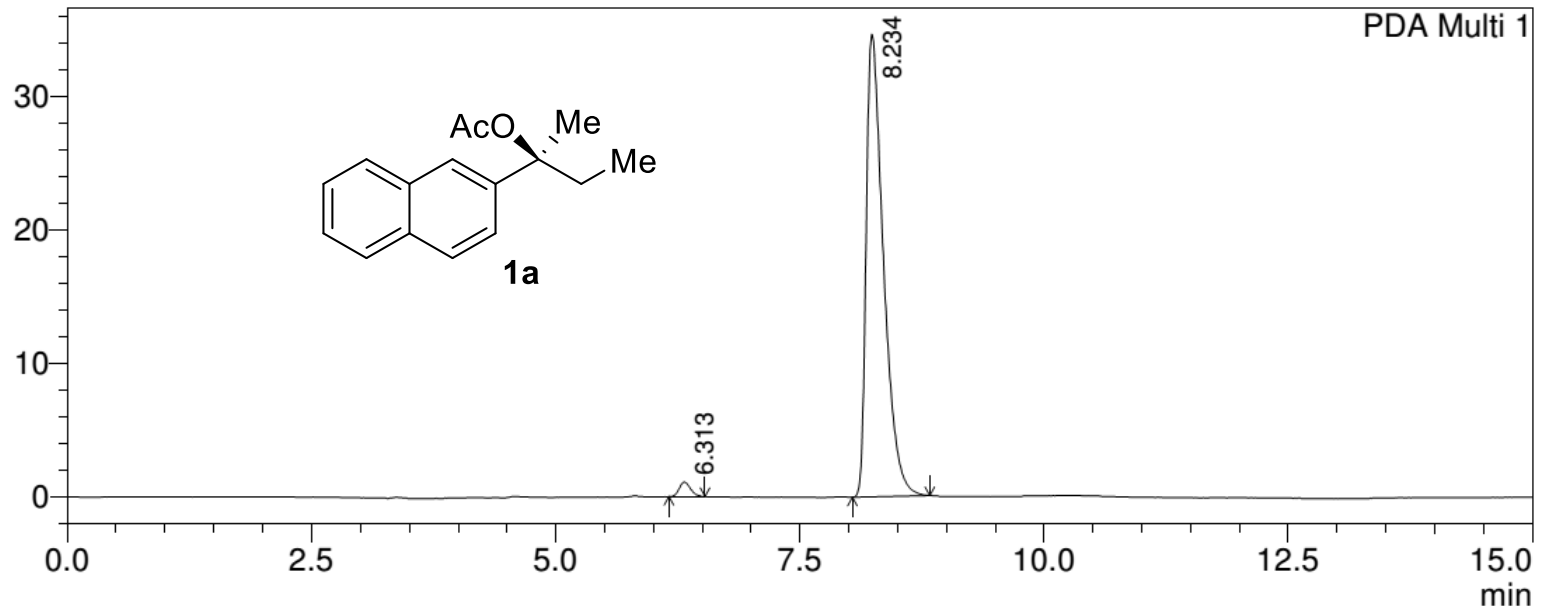

PDA Ch1 254nm 4nm

\begin{tabular}{|r|r|r|r|r|r|}
\hline \multicolumn{1}{|c|}{ Peak\# } & \multicolumn{1}{|c|}{ Ret. Time } & \multicolumn{1}{|c|}{ Area } & \multicolumn{1}{|c|}{ Height } & Area \% & \multicolumn{1}{|c|}{ Height \% } \\
\hline 1 & 6.313 & 8728 & 1084 & 2.011 & 3.036 \\
\hline 2 & 8.234 & 425380 & 34633 & 97.989 & 96.964 \\
\hline Total & & 434108 & 35717 & 100.000 & 100.000 \\
\hline
\end{tabular}


Racemic 1c

mAU

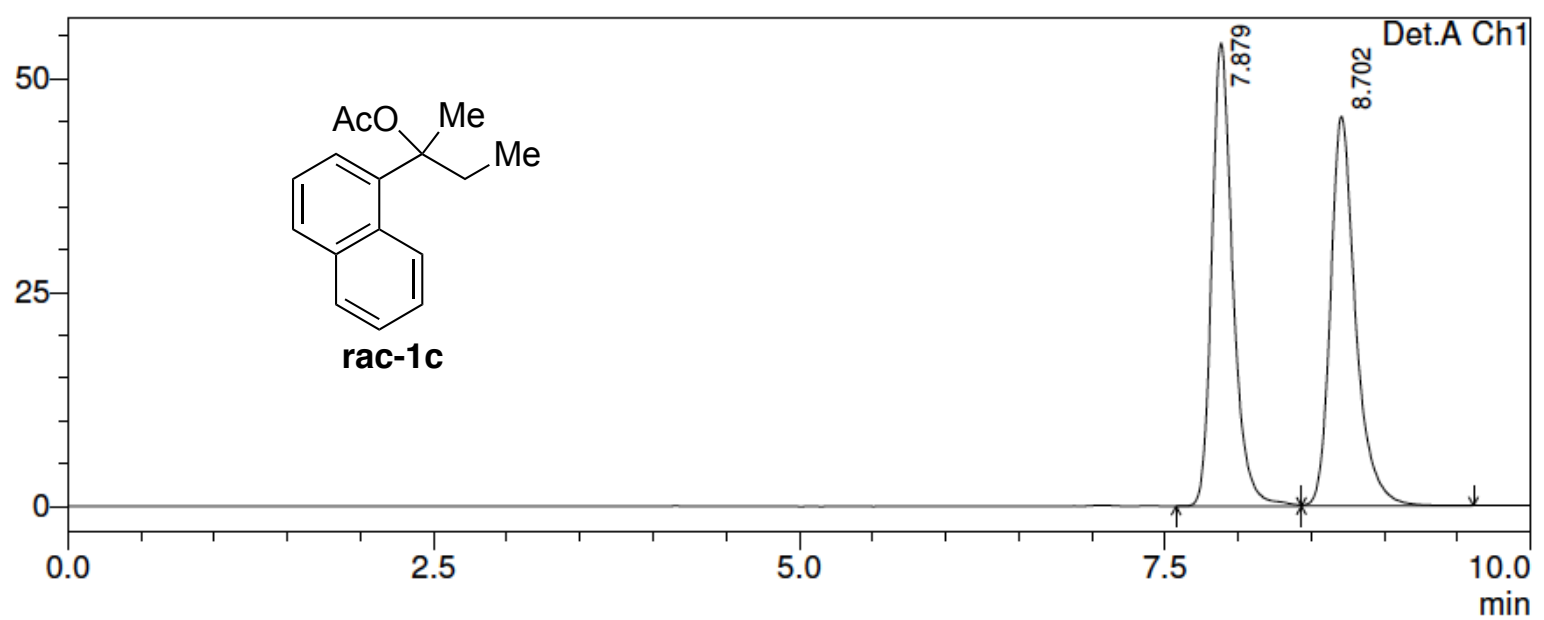

Detector A Ch1 254nm

\begin{tabular}{|r|r|r|r|r|r|}
\hline \multicolumn{1}{|c|}{ Peak\# } & Ret. Time & \multicolumn{1}{c|}{ Area } & \multicolumn{1}{c|}{ Height } & Area \% & \multicolumn{1}{c|}{ Height \% } \\
\hline 1 & 7.879 & 542497 & 54132 & 49.894 & 54.318 \\
\hline 2 & 8.702 & 544812 & 45525 & 50.106 & 45.682 \\
\hline Total & & 1087309 & 99658 & 100.000 & 100.000 \\
\hline
\end{tabular}

Enantioenriched 1c, 90\% ee

mAU

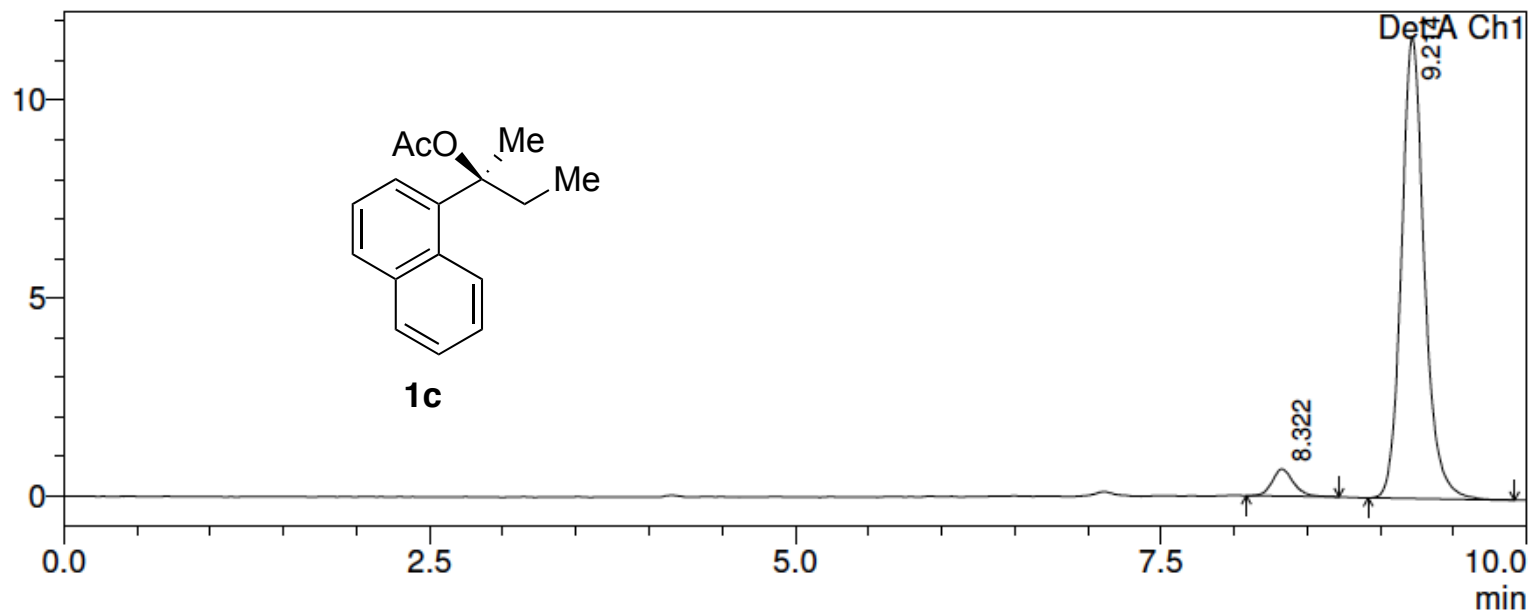

Detector A Ch1 254nm

\begin{tabular}{|r|r|r|r|r|r|}
\hline \multicolumn{1}{|c|}{ Peak\# } & \multicolumn{1}{c|}{ Ret. Time } & \multicolumn{1}{c|}{ Area } & \multicolumn{1}{c|}{ Height } & \multicolumn{1}{c|}{ Area \% } & \multicolumn{1}{c|}{ Height \% } \\
\hline 1 & 8.322 & 6955 & 681 & 5.184 & 5.523 \\
\hline 2 & 9.214 & 127204 & 11657 & 94.816 & 94.477 \\
\hline Total & & 134159 & 12338 & 100.000 & 100.000 \\
\hline
\end{tabular}


Racemic 1d

mAU

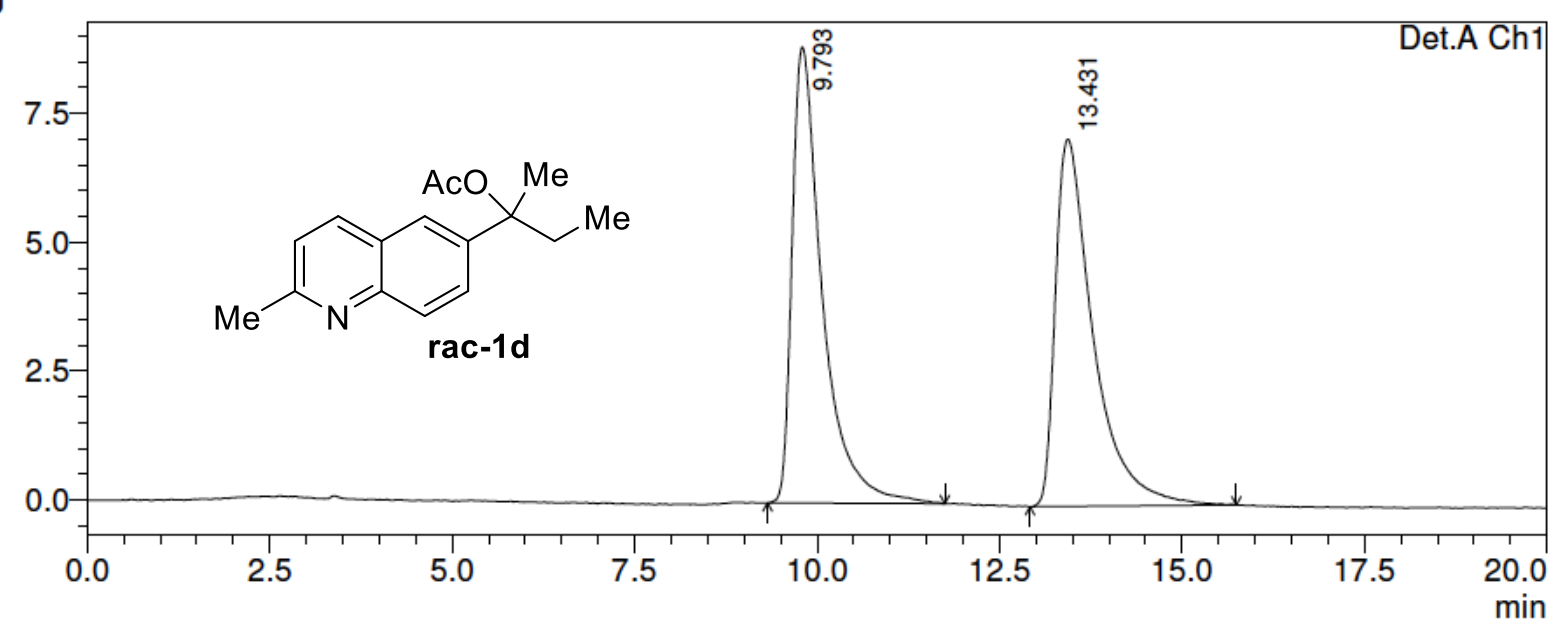

Detector A Ch1 254nm

\begin{tabular}{|r|r|r|r|r|r|}
\hline \multicolumn{1}{|c|}{ Peak\# } & Ret. Time & \multicolumn{1}{c|}{ Area } & Height & Area \% & Height \% \\
\hline 1 & 9.793 & 255341 & 8840 & 49.841 & 55.369 \\
\hline 2 & 13.431 & 256972 & 7126 & 50.159 & 44.631 \\
\hline Total & & 512313 & 15966 & 100.000 & 100.000 \\
\hline
\end{tabular}

Enantioenriched 1d, 99\% ee

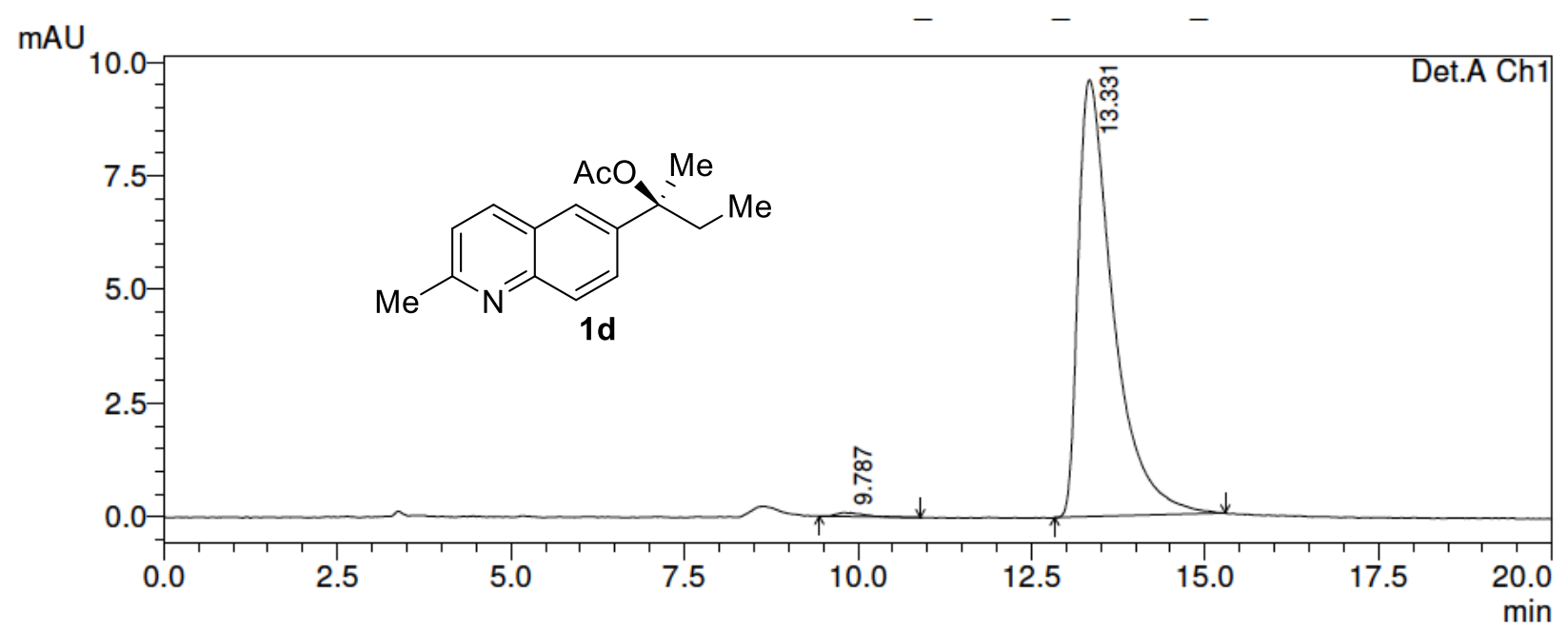

Detector A Ch1 254nm

\begin{tabular}{|r|r|r|r|r|r|}
\hline Peak\# & \multicolumn{1}{|c|}{ Ret. Time } & \multicolumn{1}{|c|}{ Area } & \multicolumn{1}{|c|}{ Height } & \multicolumn{1}{c|}{ Area \% } & \multicolumn{1}{c|}{ Height \% } \\
\hline 1 & 9.787 & 2432 & 82 & 0.716 & 0.847 \\
\hline 2 & 13.331 & 337293 & 9599 & 99.284 & 99.153 \\
\hline Total & & 339725 & 9681 & 100.000 & 100.000 \\
\hline
\end{tabular}


Racemic 1e

mAU

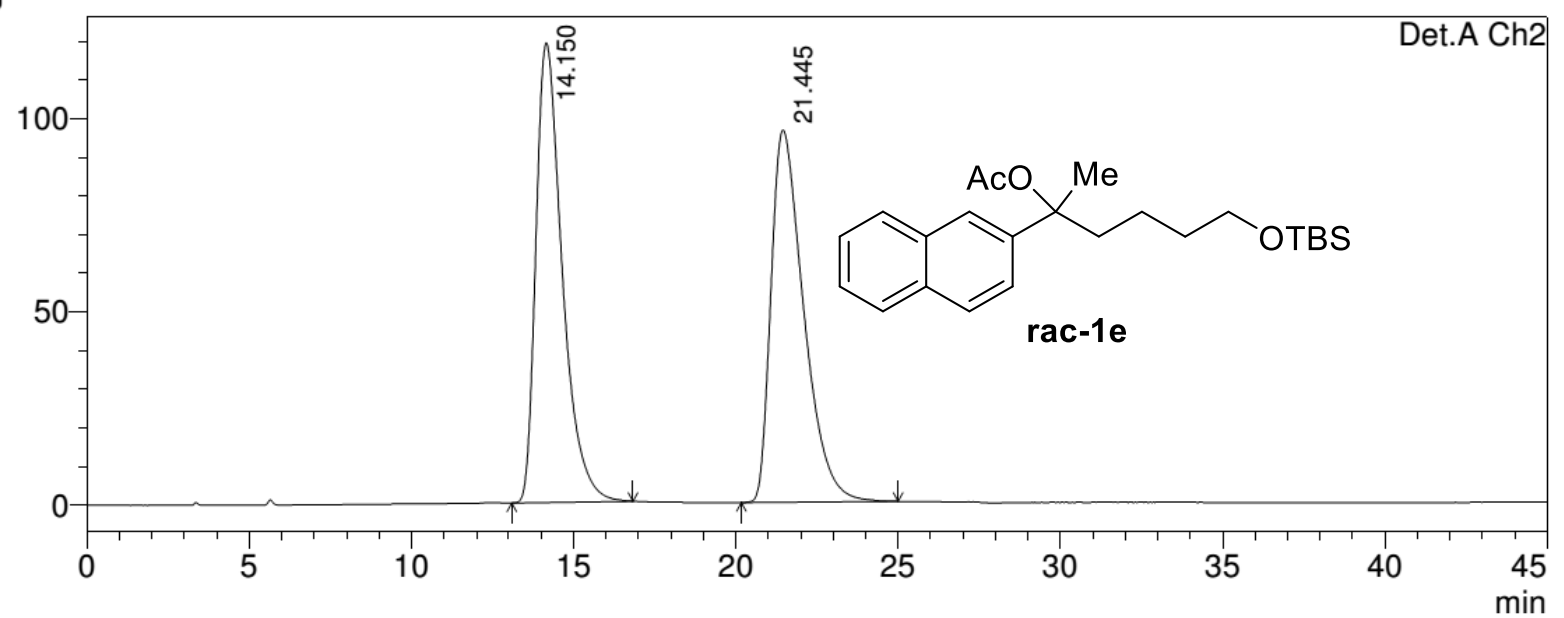

Detector A Ch2 220nm

\begin{tabular}{|r|r|r|r|r|r}
\hline \multicolumn{1}{|c|}{ Peak\# } & Ret. Time & \multicolumn{1}{c|}{ Area } & \multicolumn{1}{c|}{ Height } & \multicolumn{1}{c}{ Area \% } & \multicolumn{1}{c}{ Height \% } \\
\hline 1 & 14.150 & 6717068 & 118892 & 49.889 & 55.245 \\
\hline 2 & 21.445 & 6746910 & 96316 & 50.111 & 44.755 \\
\hline Total & & 13463978 & 215209 & 100.000 & 100.000 \\
\hline
\end{tabular}

Enantioenriched 1e, 99\% ee

mAU

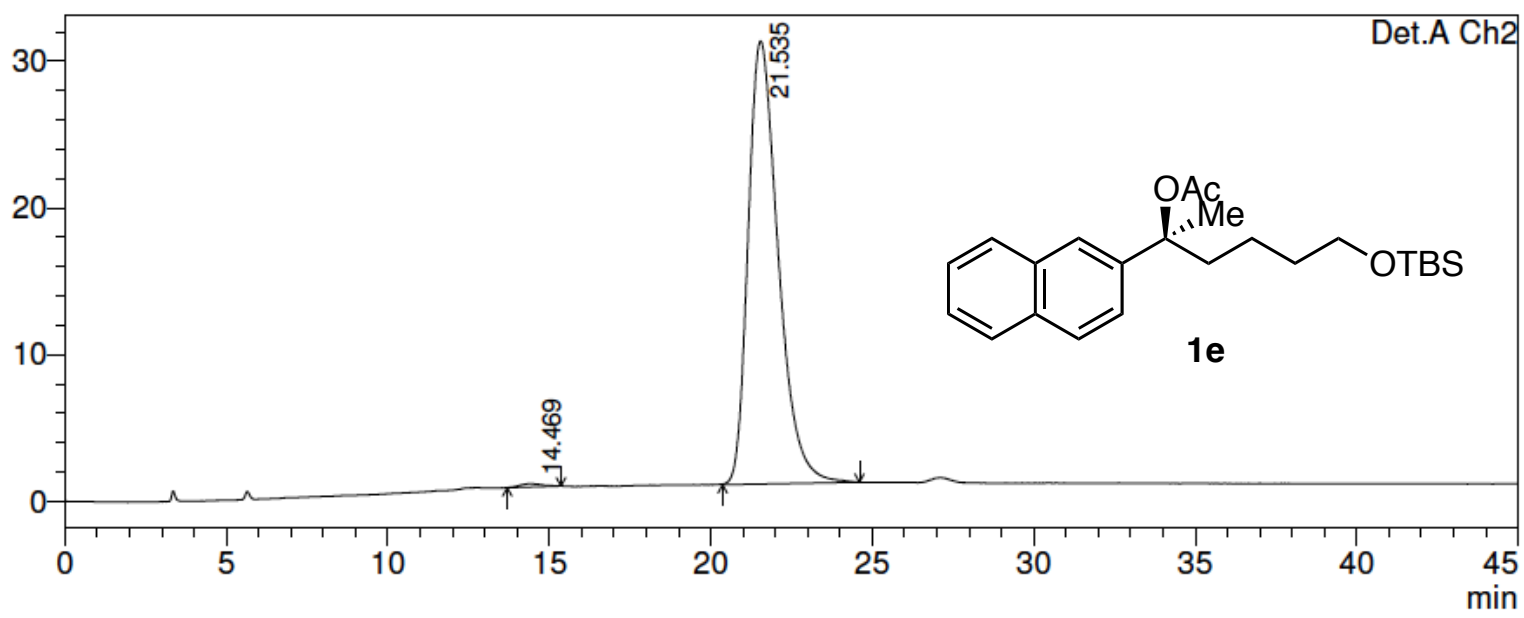

Detector A Ch2 220nm

\begin{tabular}{|r|r|r|r|r|r|}
\hline \multicolumn{1}{|c|}{ Peak\# } & Ret. Time & \multicolumn{1}{c|}{ Area } & \multicolumn{1}{c|}{ Height } & Area \% & Height \% \\
\hline 1 & 14.469 & 10257 & 216 & 0.521 & 0.711 \\
\hline 2 & 21.535 & 1957089 & 30206 & 99.479 & 99.289 \\
\hline Total & & 1967346 & 30422 & 100.000 & 100.000 \\
\hline
\end{tabular}


Racemic $\mathbf{1 f}$

mAU

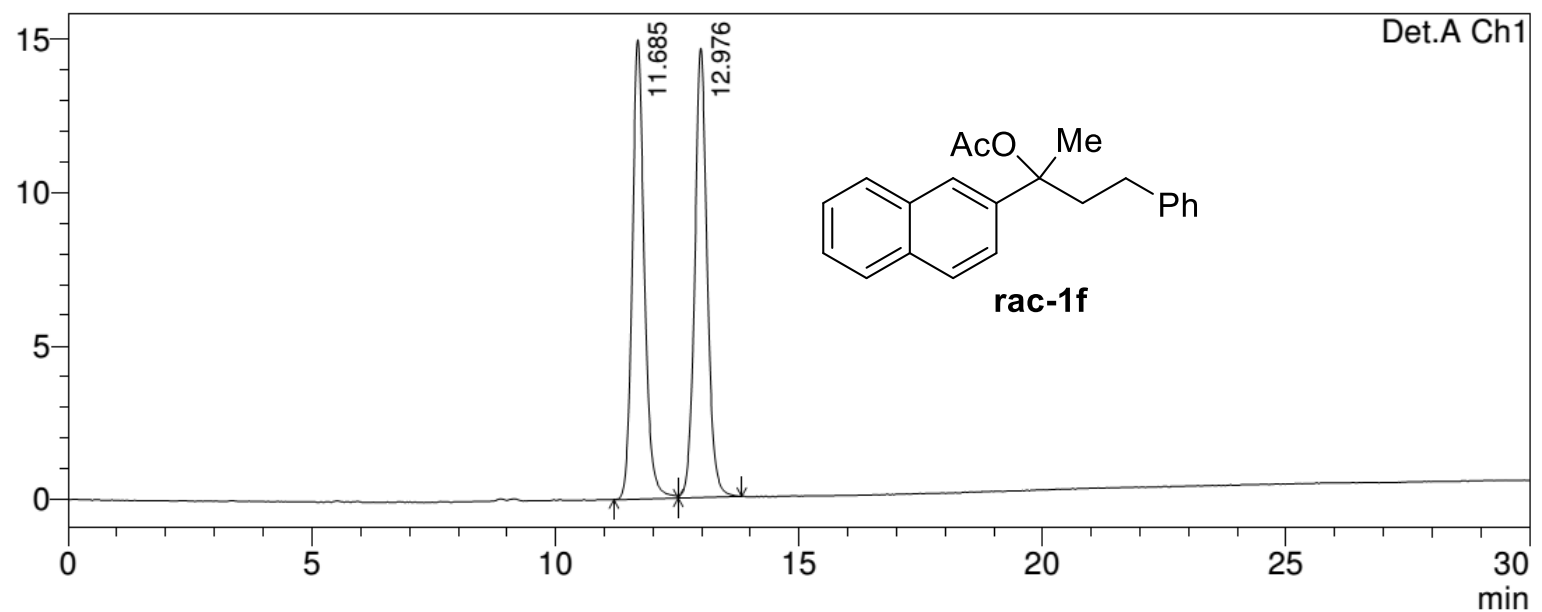

Detector A Ch1 254nm

\begin{tabular}{|r|r|r|r|r|r|}
\hline \multicolumn{1}{|c|}{ Peak\# } & Ret. Time & Area & Height & Area \% & \multicolumn{1}{c|}{ Height \% } \\
\hline 1 & 11.685 & 259259 & 14975 & 49.898 & 50.568 \\
\hline 2 & 12.976 & 260324 & 14639 & 50.102 & 49.432 \\
\hline Total & & 519583 & 29614 & 100.000 & 100.000 \\
\hline
\end{tabular}

Enantioenriched 1f, 94\% ee

mAU

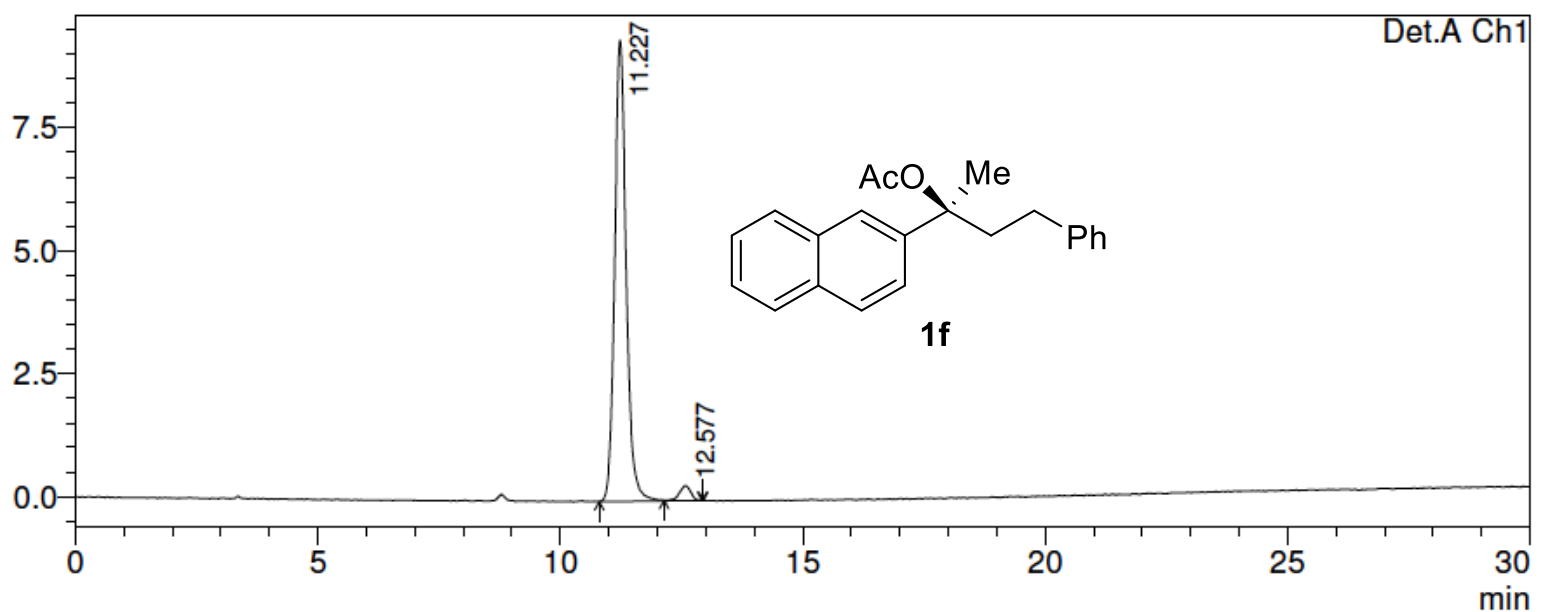

Detector A Ch1 254nm

\begin{tabular}{|r|r|r|r|r|r|}
\hline \multicolumn{1}{|c|}{ Peak\# } & \multicolumn{1}{|c|}{ Ret. Time } & \multicolumn{1}{|c|}{ Area } & \multicolumn{1}{c|}{ Height } & Area \% & \multicolumn{1}{|c|}{ Height \% } \\
\hline 1 & 11.227 & 154894 & 9370 & 97.078 & 96.964 \\
\hline 2 & 12.577 & 4663 & 293 & 2.922 & 3.036 \\
\hline Total & & 159557 & 9664 & 100.000 & 100.000 \\
\hline
\end{tabular}


Racemic 1g

mAU

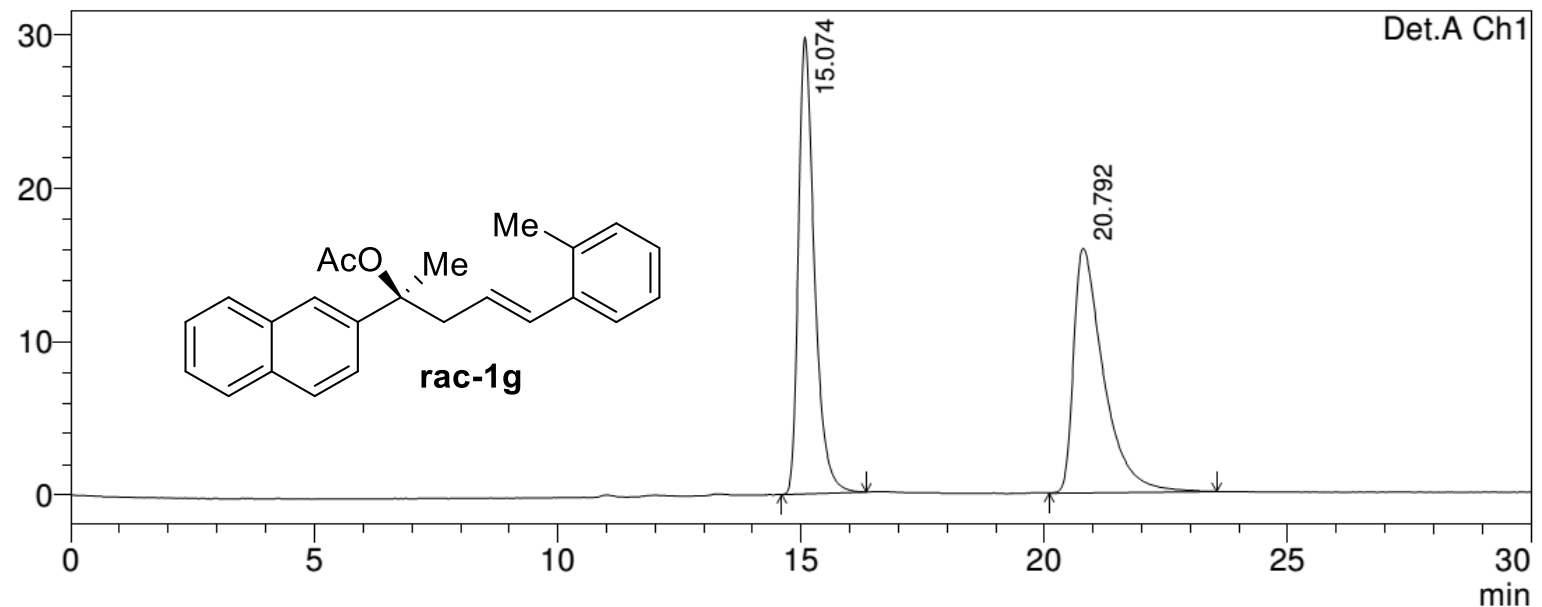

Detector A Ch1 254nm

\begin{tabular}{|r|r|r|r|r|r|}
\hline Peak\# & Ret. Time & \multicolumn{1}{c|}{ Area } & Height & Area \% & \multicolumn{1}{c|}{ Height \% } \\
\hline 1 & 15.074 & 682100 & 29840 & 49.883 & 65.138 \\
\hline 2 & 20.792 & 685307 & 15970 & 50.117 & 34.862 \\
\hline Total & & 1367408 & 45811 & 100.000 & 100.000 \\
\hline
\end{tabular}

Enantioenriched 1g, 96\% ee

mAU

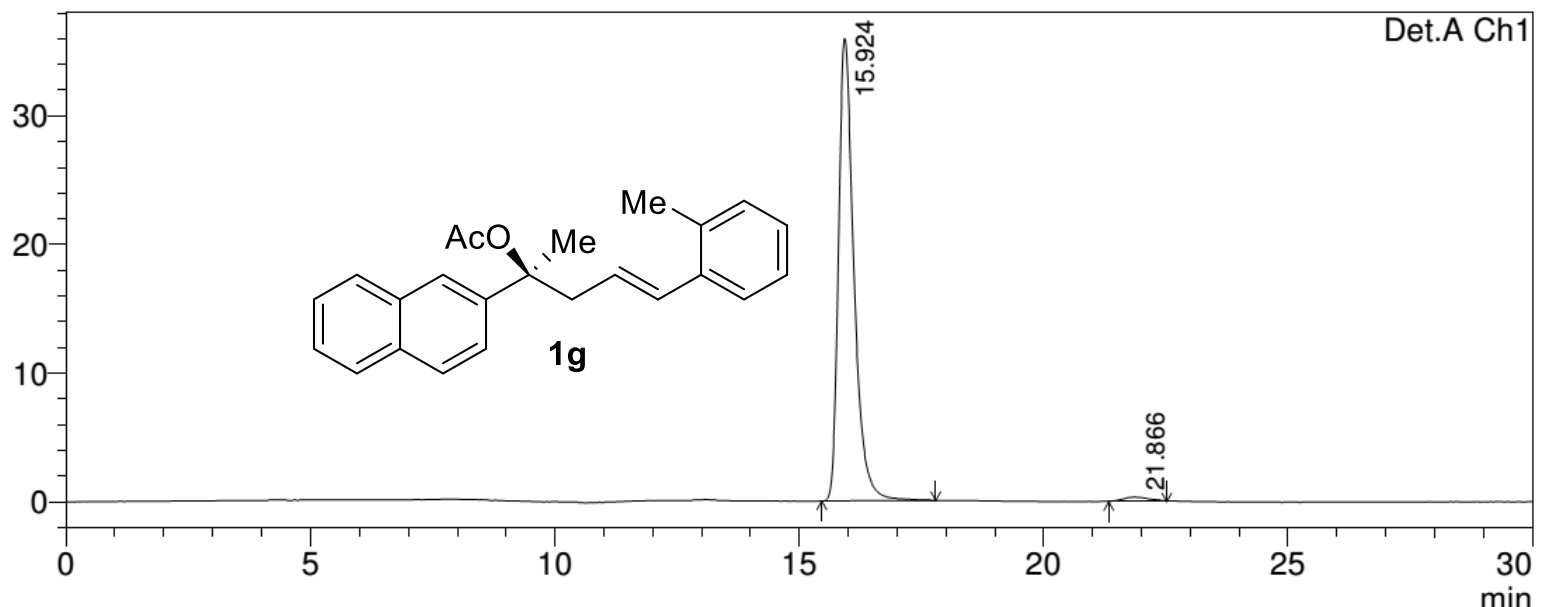

Detector A Ch1 254nm

\begin{tabular}{|r|r|r|r|r|r|}
\hline \multicolumn{1}{|c|}{ Peak\# } & Ret. Time & \multicolumn{1}{c|}{ Area } & Height & Area \% & \multicolumn{1}{c|}{ Height \% } \\
\hline 1 & 15.924 & 789682 & 35965 & 98.270 & 99.044 \\
\hline 2 & 21.866 & 13905 & 347 & 1.730 & 0.956 \\
\hline Total & & 803587 & 36312 & 100.000 & 100.000 \\
\hline
\end{tabular}


Racemic 1i

mAU

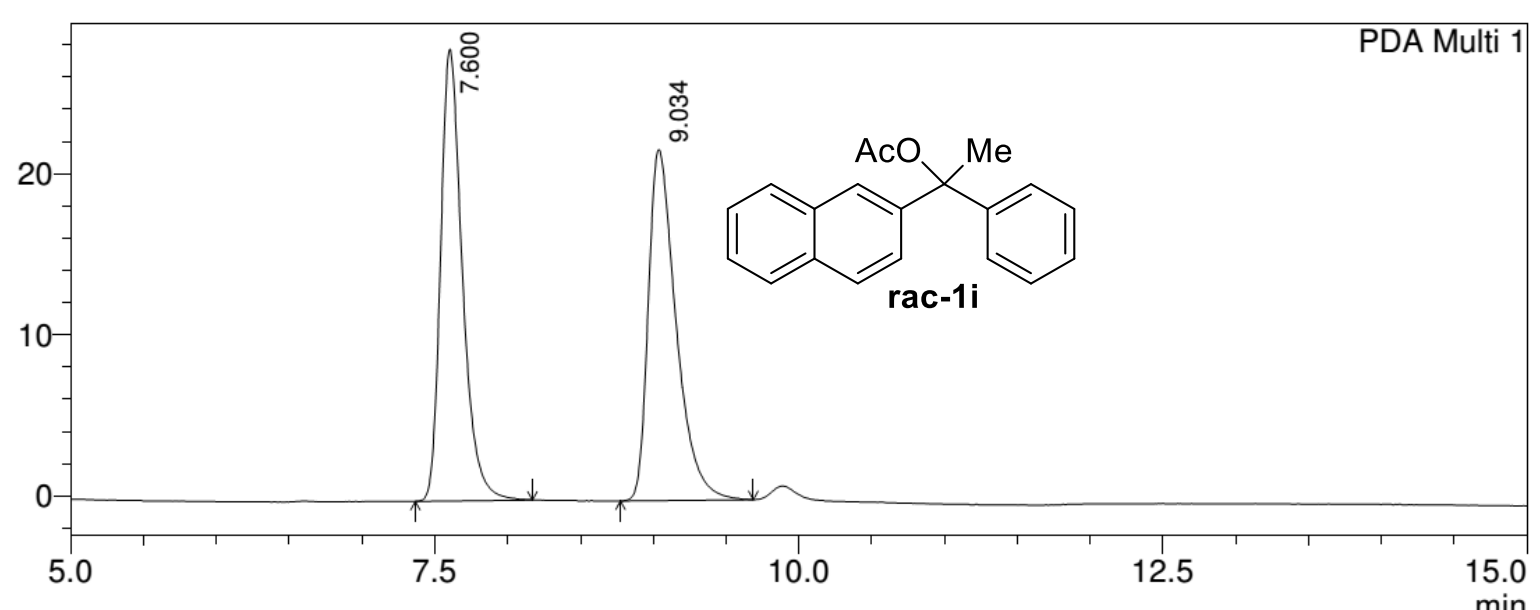

PDA Ch1 254nm 4nm

\begin{tabular}{|r|r|r|r|r|r|}
\hline Peak\# & Ret. Time & \multicolumn{1}{c|}{ Area } & Height & \multicolumn{1}{|c|}{ Area \% } & Height \% \\
\hline 1 & 7.600 & 291470 & 28014 & 50.165 & 56.265 \\
\hline 2 & 9.034 & 289554 & 21776 & 49.835 & 43.735 \\
\hline Total & & 581024 & 49790 & 100.000 & 100.000 \\
\hline
\end{tabular}

Enantioenriched 1i, 96\% ee

mAU

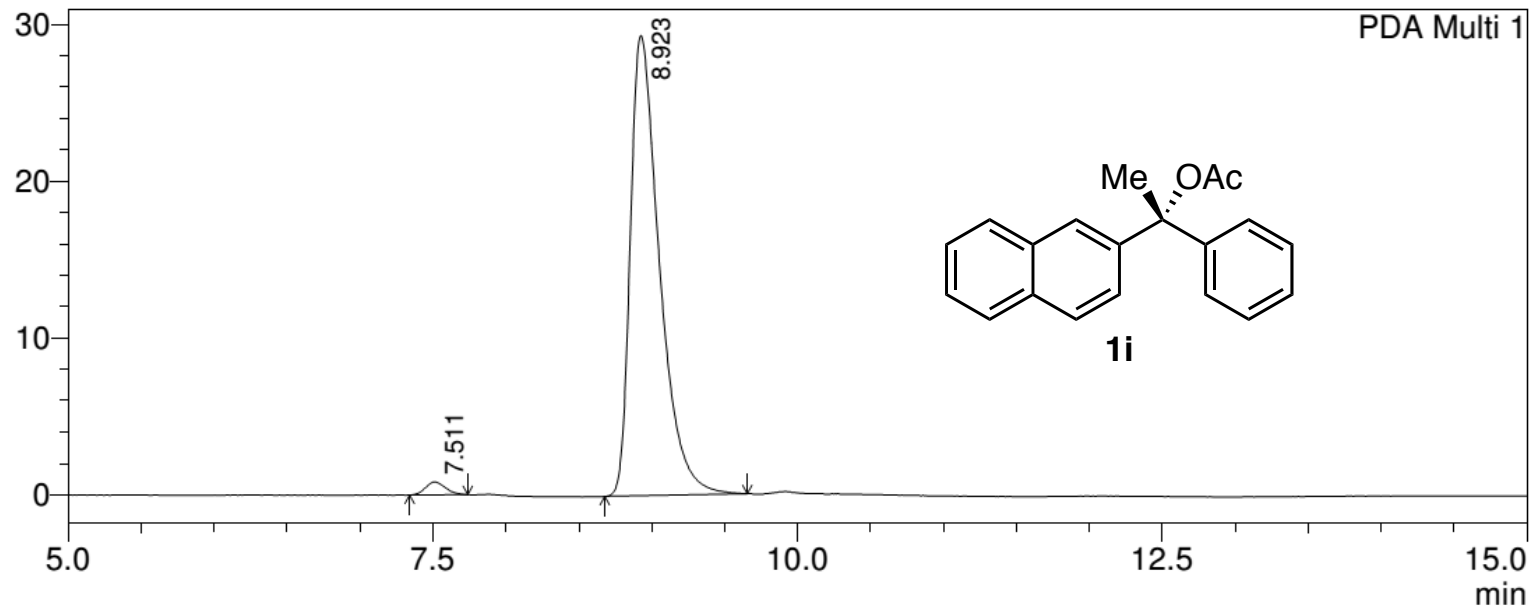

PDA Ch1 254nm 4nm

\begin{tabular}{|r|r|r|r|r|r}
\hline \multicolumn{1}{|c|}{ Peak\# } & \multicolumn{1}{c|}{ Ret. Time } & \multicolumn{1}{c|}{ Area } & \multicolumn{1}{c|}{ Height } & \multicolumn{1}{c|}{ Area \% } & \multicolumn{1}{c}{ Height \% } \\
\hline 1 & 7.511 & 7964 & 824 & 1.915 & 2.731 \\
\hline 2 & 8.923 & 407977 & 29359 & 98.085 & 97.269 \\
\hline Total & & 415942 & 30183 & 100.000 & 100.000 \\
\hline
\end{tabular}


Racemic S-1a

mAU

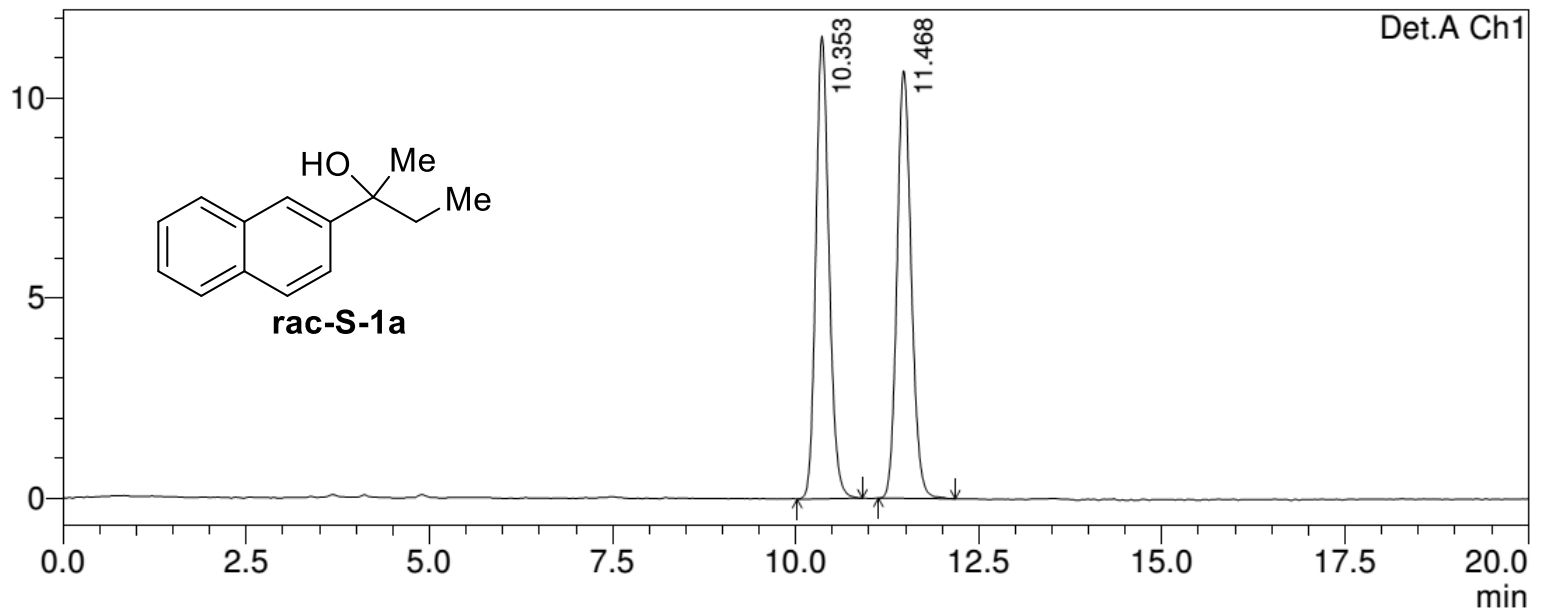

Detector A Ch1 254nm

\begin{tabular}{|r|r|r|r|r|r|}
\hline \multicolumn{1}{|c|}{ Peak\# } & Ret. Time & \multicolumn{1}{|c|}{ Area } & Height & Area \% & Height \% \\
\hline 1 & 10.353 & 144648 & 11554 & 49.962 & 51.955 \\
\hline 2 & 11.468 & 144869 & 10684 & 50.038 & 48.045 \\
\hline Total & & 289518 & 22238 & 100.000 & 100.000 \\
\hline
\end{tabular}

Enantioenriched 1a, 96\% ee $\mathrm{mAU}$

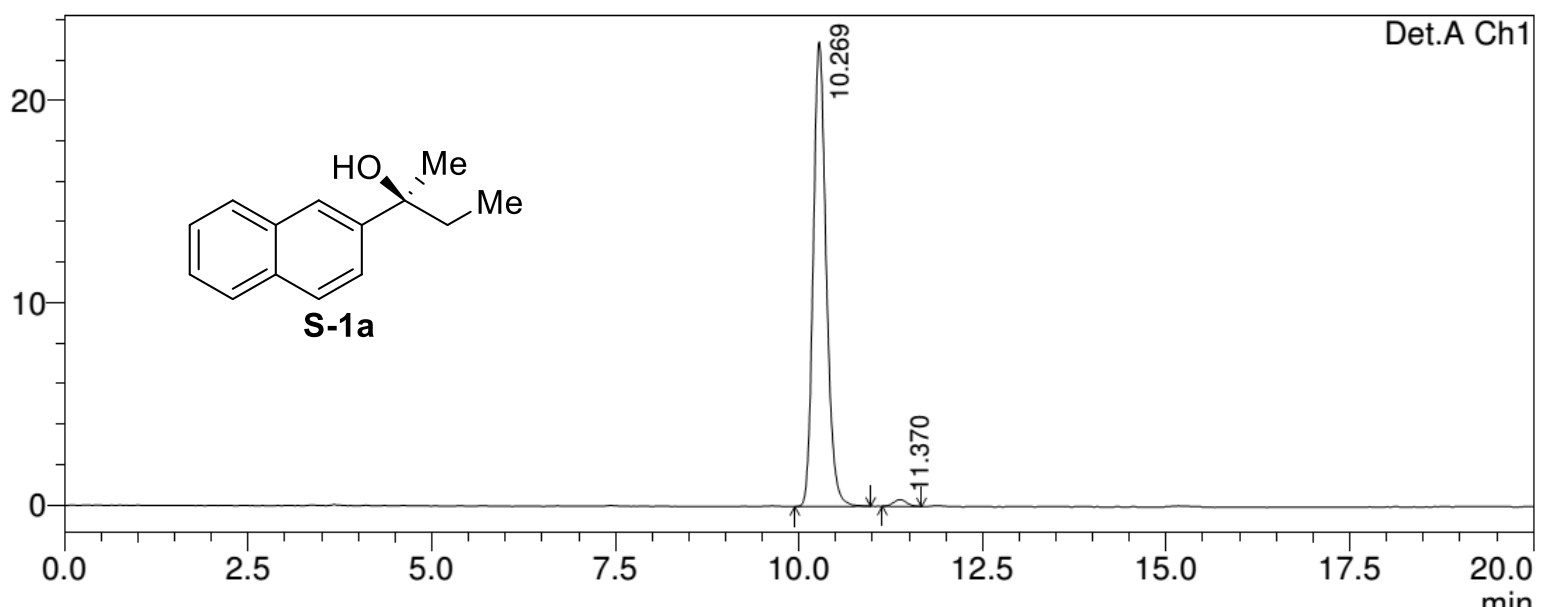

Detector A Ch1 254nm

\begin{tabular}{|r|r|r|r|r|r|}
\hline \multicolumn{1}{|c|}{ Peak\# } & \multicolumn{1}{c|}{ Ret. Time } & \multicolumn{1}{c|}{ Area } & \multicolumn{1}{c|}{ Height } & Area \% & \multicolumn{1}{c|}{ Height \% } \\
\hline 1 & 10.269 & 283433 & 22977 & 98.493 & 98.537 \\
\hline 2 & 11.370 & 4338 & 341 & 1.507 & 1.463 \\
\hline Total & & 287771 & 23318 & 100.000 & 100.000 \\
\hline
\end{tabular}


Racemic S-1b

$\mathrm{mAU}$

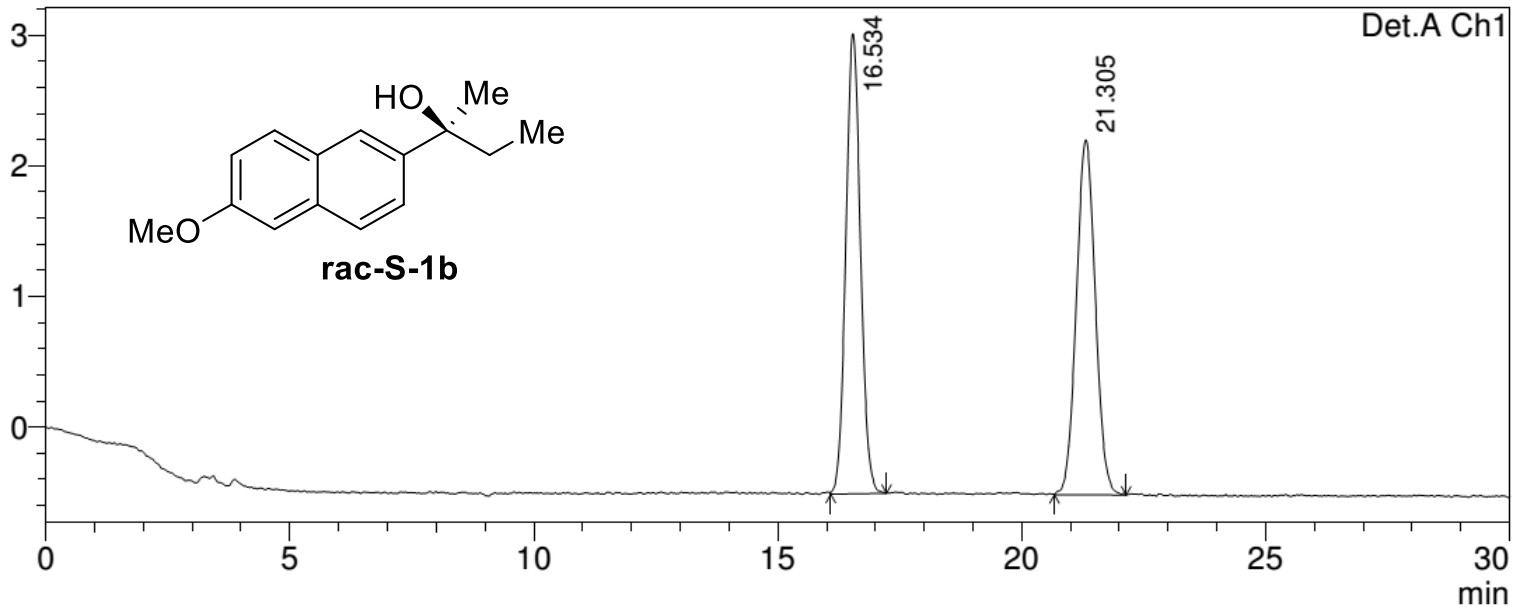

Detector A Ch1 254nm

\begin{tabular}{|r|r|r|r|r|r|}
\hline Peak\# & Ret. Time & \multicolumn{1}{c|}{ Area } & \multicolumn{1}{c|}{ Height } & Area \% & Height \% \\
\hline 1 & 16.534 & 73547 & 3522 & 50.022 & 56.460 \\
\hline 2 & 21.305 & 73483 & 2716 & 49.978 & 43.540 \\
\hline Total & & 147031 & 6238 & 100.000 & 100.000 \\
\hline
\end{tabular}

Enantioenriched S-1b, 91\% ee

mAU

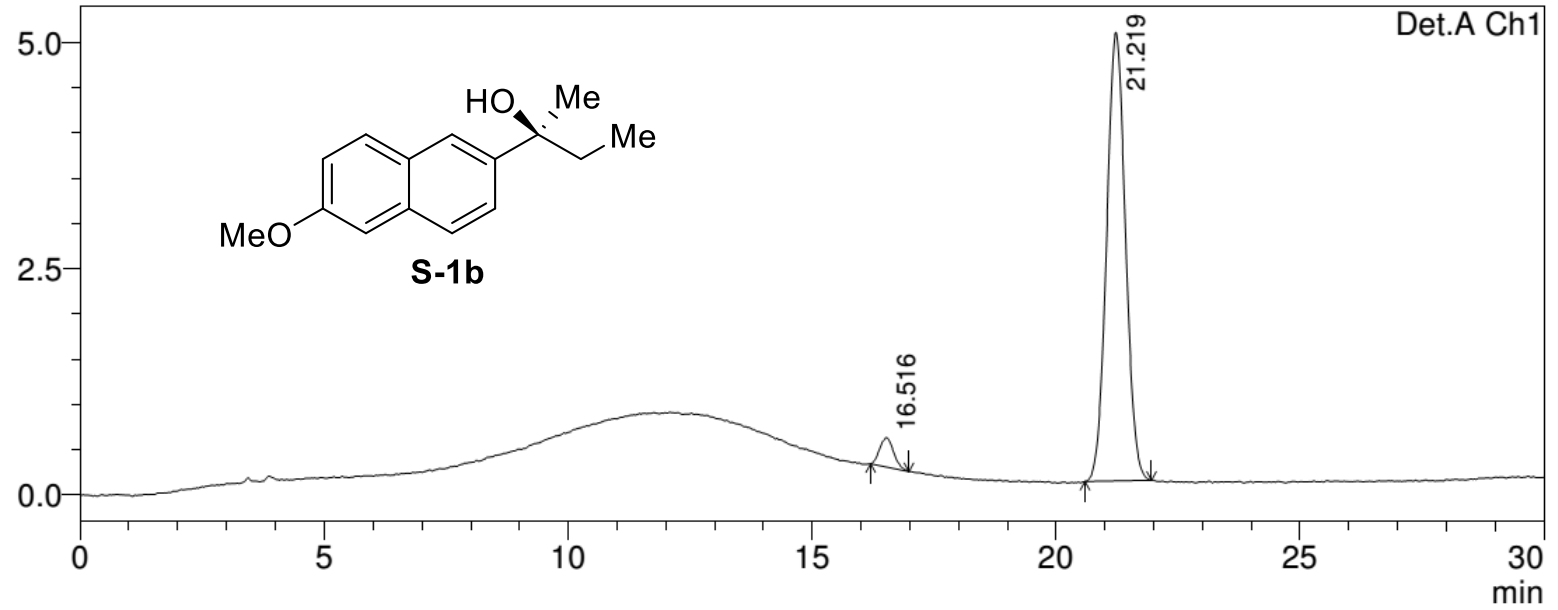

Detector A Ch1 254nm

\begin{tabular}{|r|r|r|r|r|r|}
\hline \multicolumn{1}{|c|}{ Peak\# } & Ret. Time & \multicolumn{1}{c|}{ Area } & \multicolumn{1}{c|}{ Height } & Area \% & Height \% \\
\hline 1 & 16.516 & 6536 & 328 & 4.672 & 6.201 \\
\hline 2 & 21.219 & 133384 & 4962 & 95.328 & 93.799 \\
\hline Total & & 139920 & 5291 & 100.000 & 100.000 \\
\hline
\end{tabular}


Racemic S-1c

mAU

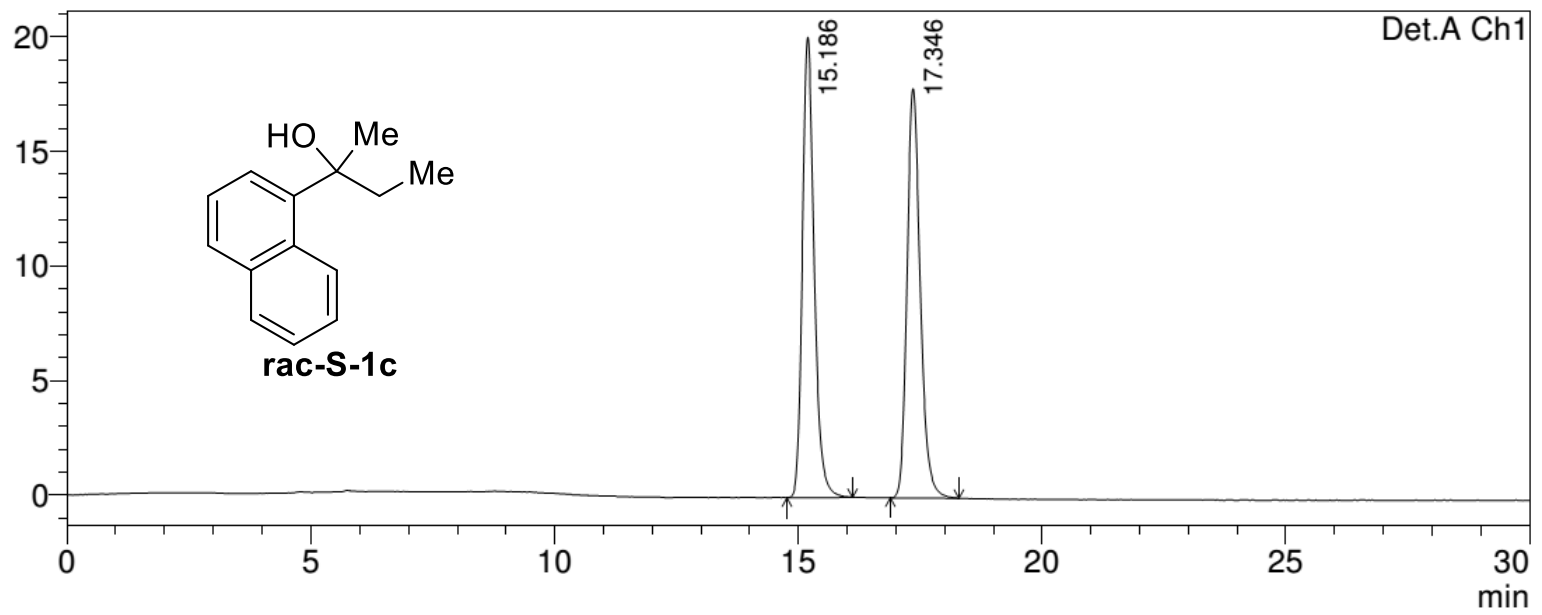

Detector A Ch1 254nm

\begin{tabular}{|r|r|r|r|r|r|}
\hline \multicolumn{1}{|c|}{ Peak\# } & Ret. Time & \multicolumn{1}{|c|}{ Area } & Height & Area \% & Height \% \\
\hline 1 & 15.186 & 337510 & 20102 & 49.967 & 52.913 \\
\hline 2 & 17.346 & 337952 & 17889 & 50.033 & 47.087 \\
\hline Total & & 675461 & 37991 & 100.000 & 100.000 \\
\hline
\end{tabular}

Enantioenriched S-1c, 90\% ee

$\mathrm{mAU}$

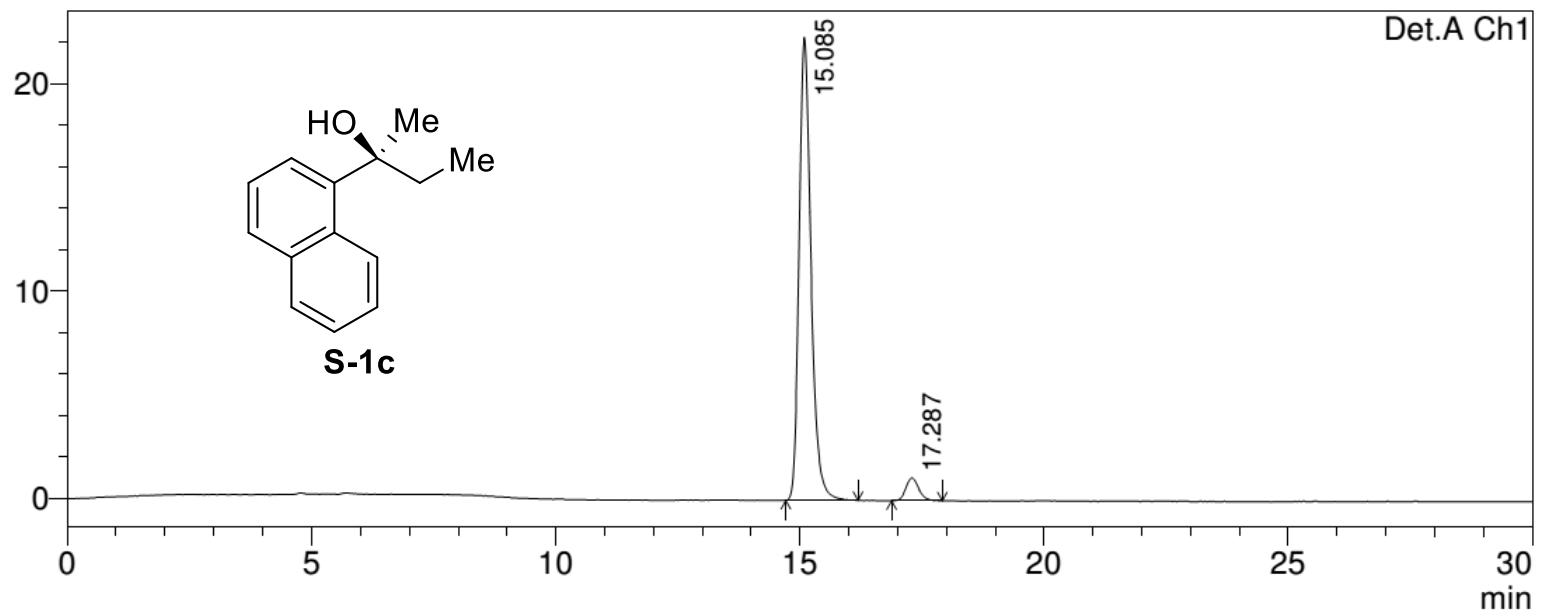

Detector A Ch1 254nm

\begin{tabular}{|r|r|r|r|r|r|}
\hline Peak\# & Ret. Time & \multicolumn{1}{|c|}{ Area } & Height & Area \% & \multicolumn{1}{|c|}{ Height \% } \\
\hline 1 & 15.085 & 379069 & 22316 & 94.789 & 95.245 \\
\hline 2 & 17.287 & 20837 & 1114 & 5.211 & 4.755 \\
\hline Total & & 399906 & 23430 & 100.000 & 100.000 \\
\hline
\end{tabular}


Racemic S-1d

mAU

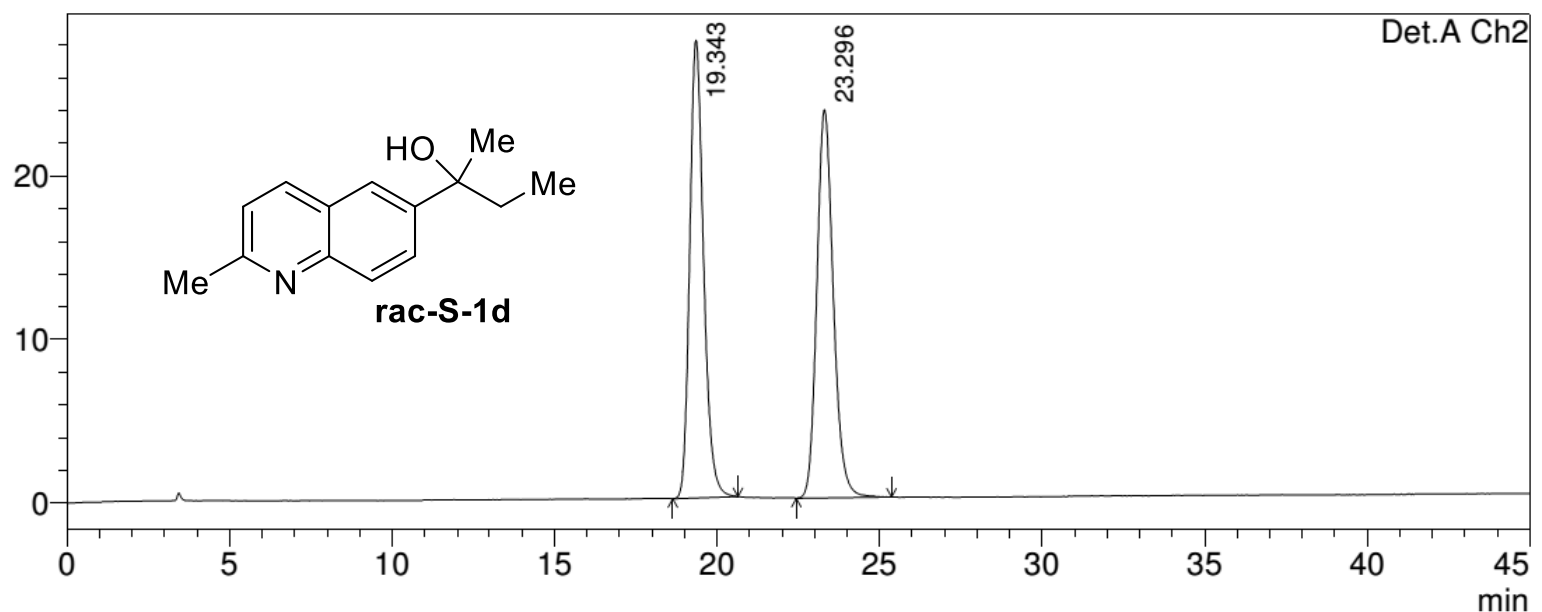

Detector A Ch2 230nm

\begin{tabular}{|r|r|r|r|r|r|}
\hline \multicolumn{1}{|c|}{ Peak\# } & Ret. Time & \multicolumn{1}{c|}{ Area } & Height & Area \% & \multicolumn{1}{|c|}{ Height \% } \\
\hline 1 & 19.343 & 830508 & 27995 & 49.756 & 54.100 \\
\hline 2 & 23.296 & 838670 & 23752 & 50.244 & 45.900 \\
\hline Total & & 1669178 & 51747 & 100.000 & 100.000 \\
\hline
\end{tabular}

Enantioenriched S-1d, 99\% ee $\mathrm{mAU}$

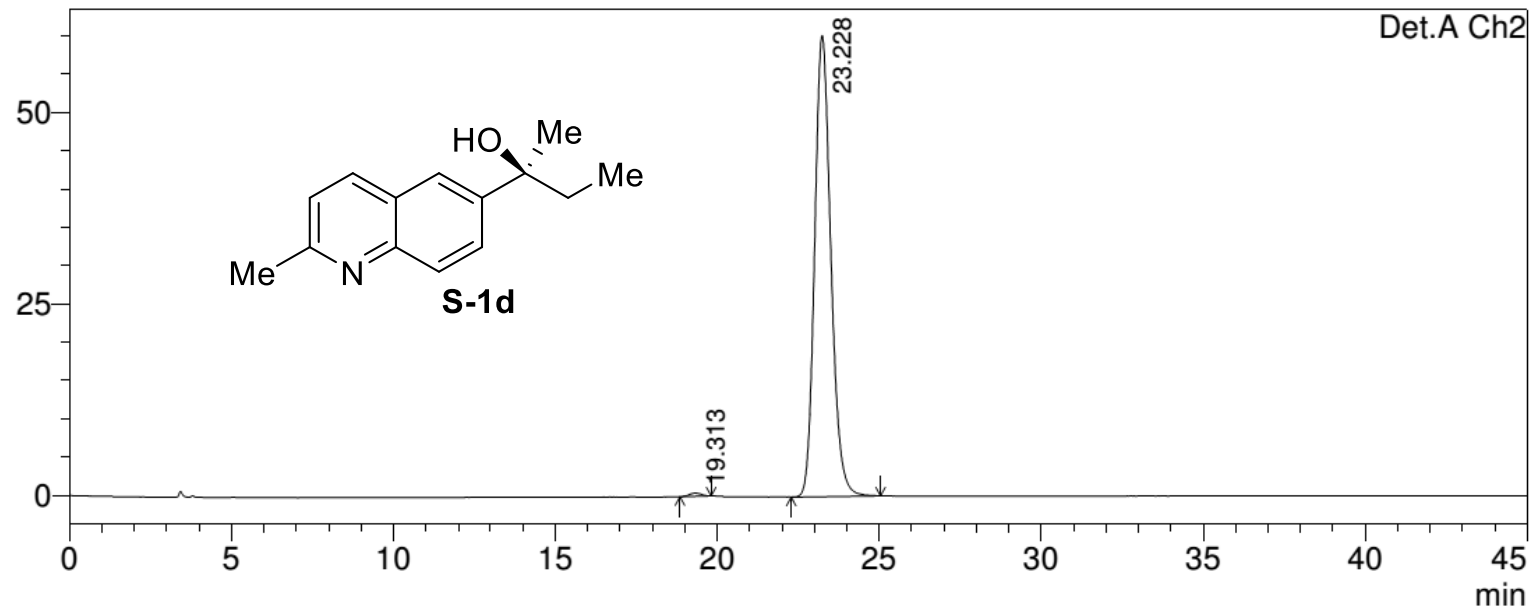

Detector A Ch2 230nm

\begin{tabular}{|r|r|r|r|r|r|}
\hline \multicolumn{1}{|c|}{ Peak\# } & Ret. Time & \multicolumn{1}{c|}{ Area } & \multicolumn{1}{c|}{ Height } & Area \% & \multicolumn{1}{c|}{ Height \% } \\
\hline 1 & 19.313 & 10754 & 429 & 0.510 & 0.708 \\
\hline 2 & 23.228 & 2099723 & 60224 & 99.490 & 99.292 \\
\hline Total & & 2110477 & 60653 & 100.000 & 100.000 \\
\hline
\end{tabular}


Racemic S-1e

mAU

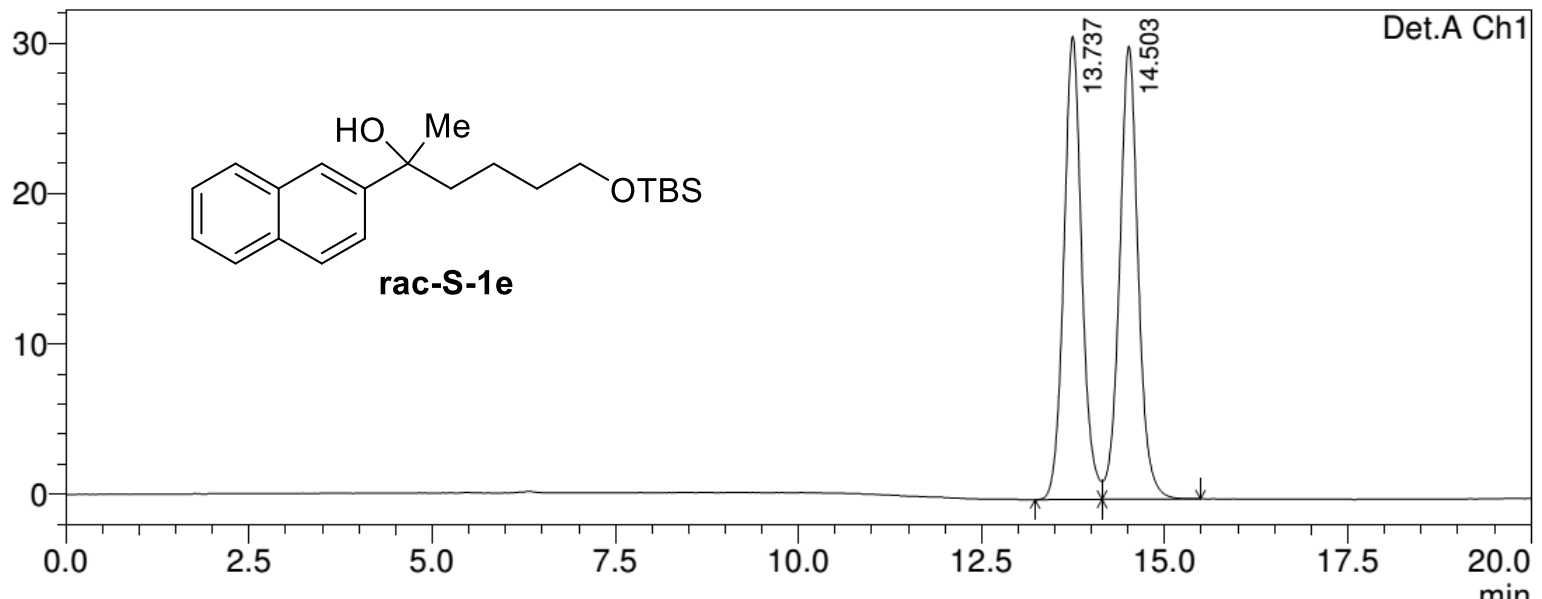

Detector A Ch1 254nm

\begin{tabular}{|r|r|r|r|r|r|}
\hline \multicolumn{1}{|c|}{ Peak\# } & Ret. Time & \multicolumn{1}{c|}{ Area } & Height & Area \% & Height \% \\
\hline 1 & 13.737 & 525563 & 30811 & 49.594 & 50.552 \\
\hline 2 & 14.503 & 534166 & 30139 & 50.406 & 49.448 \\
\hline Total & & 1059729 & 60950 & 100.000 & 100.000 \\
\hline
\end{tabular}

Enantioenriched S-1e, 99\% ee $\mathrm{mAU}$

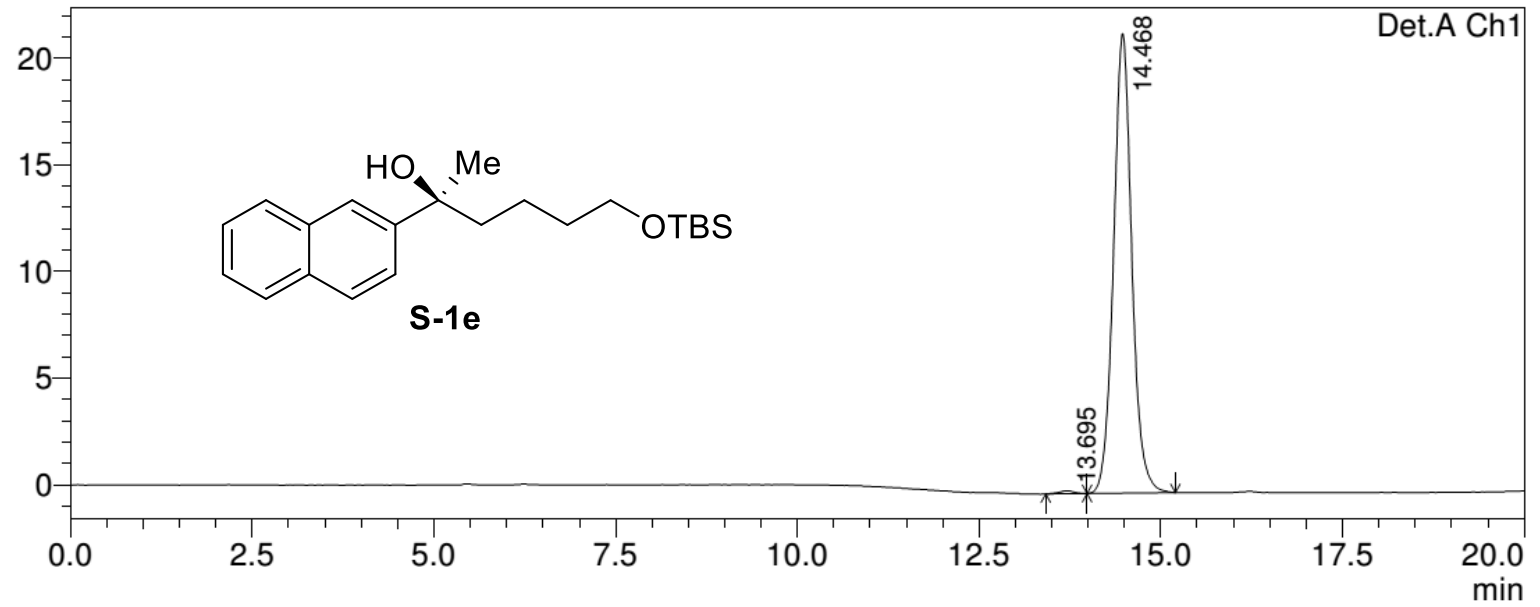

Detector A Ch1 254nm

\begin{tabular}{|r|r|r|r|r|r|}
\hline \multicolumn{1}{|c|}{ Peak\# } & Ret. Time & \multicolumn{1}{|c|}{ Area } & \multicolumn{1}{|c|}{ Height } & Area \% & \multicolumn{1}{c|}{ Height \% } \\
\hline 1 & 13.695 & 2096 & 141 & 0.553 & 0.651 \\
\hline 2 & 14.468 & 376900 & 21525 & 99.447 & 99.349 \\
\hline Total & & 378996 & 21666 & 100.000 & 100.000 \\
\hline
\end{tabular}


Racemic 2-(Naphthalen-2-yl)pent-4-en-2-ol (used in preparation of S-1g) mAU

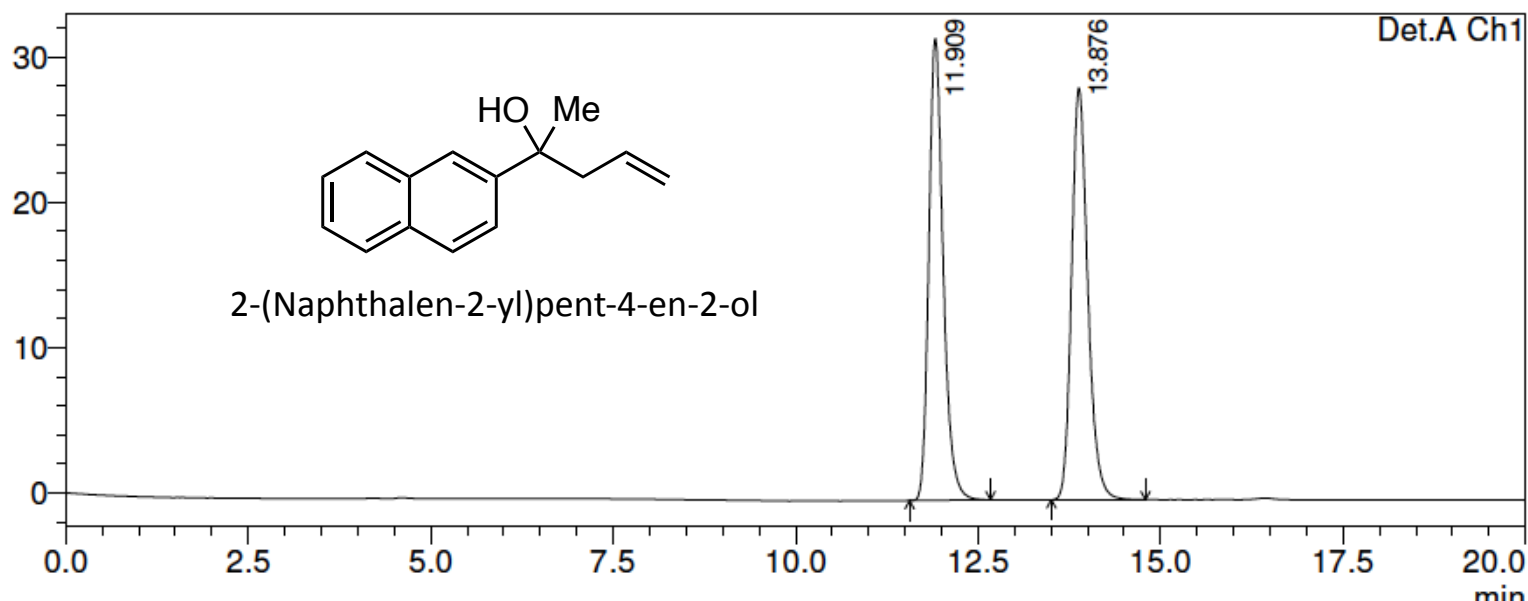

Detector A Ch1 254nm

\begin{tabular}{|r|r|r|r|r|r|}
\hline Peak\# & Ret. Time & \multicolumn{1}{|c|}{ Area } & Height & \multicolumn{1}{|c|}{ Area \% } & \multicolumn{1}{c|}{ Height \% } \\
\hline 1 & 11.909 & 435852 & 31788 & 49.790 & 52.848 \\
\hline 2 & 13.876 & 439530 & 28362 & 50.210 & 47.152 \\
\hline Total & & 875382 & 60150 & 100.000 & 100.000 \\
\hline
\end{tabular}

Enantioenriched 2-(Naphthalen-2-yl)pent-4-en-2-ol (used in preparation of S-1g), 96\% ee $\mathrm{mAU}$

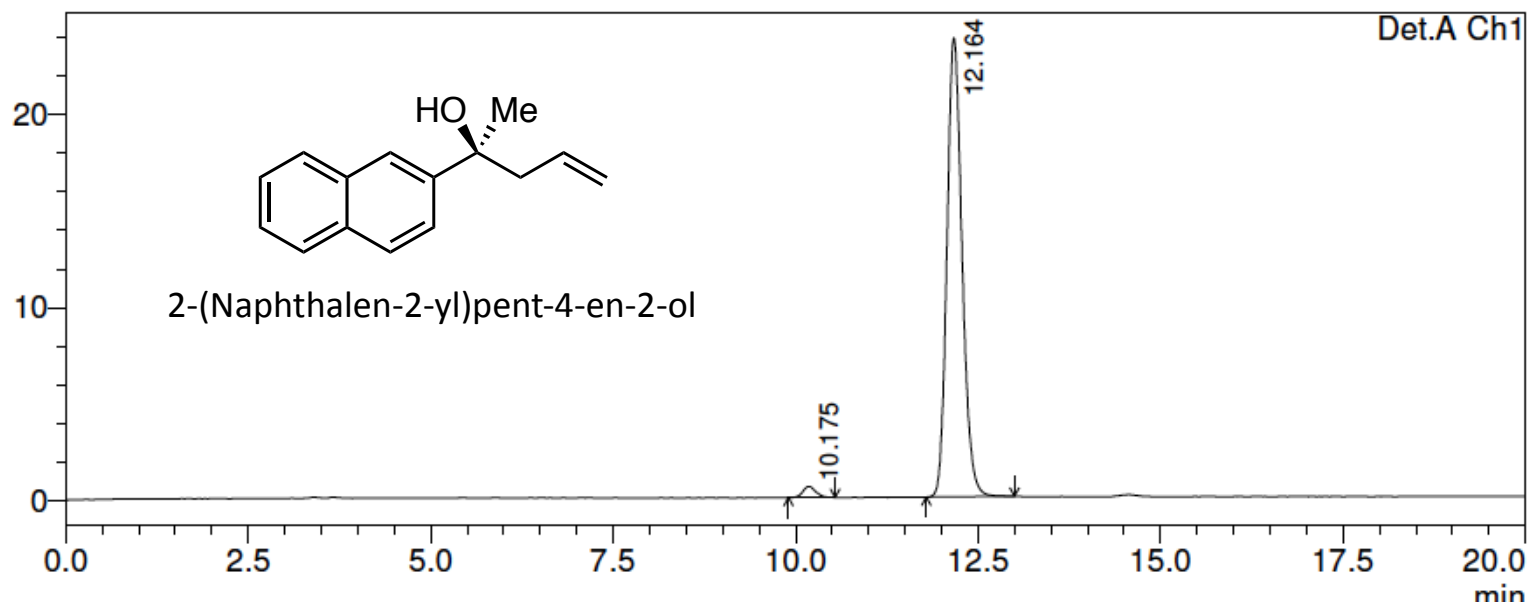

Detector A Ch1 254nm

\begin{tabular}{|r|r|r|r|r|r|}
\hline Peak\# & \multicolumn{1}{|c|}{ Ret. Time } & \multicolumn{1}{|c|}{ Area } & \multicolumn{1}{c|}{ Height } & \multicolumn{1}{c|}{ Area \% } & Height \% \\
\hline 1 & 10.175 & 7104 & 570 & 2.024 & 2.336 \\
\hline 2 & 12.164 & 343811 & 23822 & 97.976 & 97.664 \\
\hline Total & & 350915 & 24392 & 100.000 & 100.000 \\
\hline
\end{tabular}


Racemic S-1h

mAU

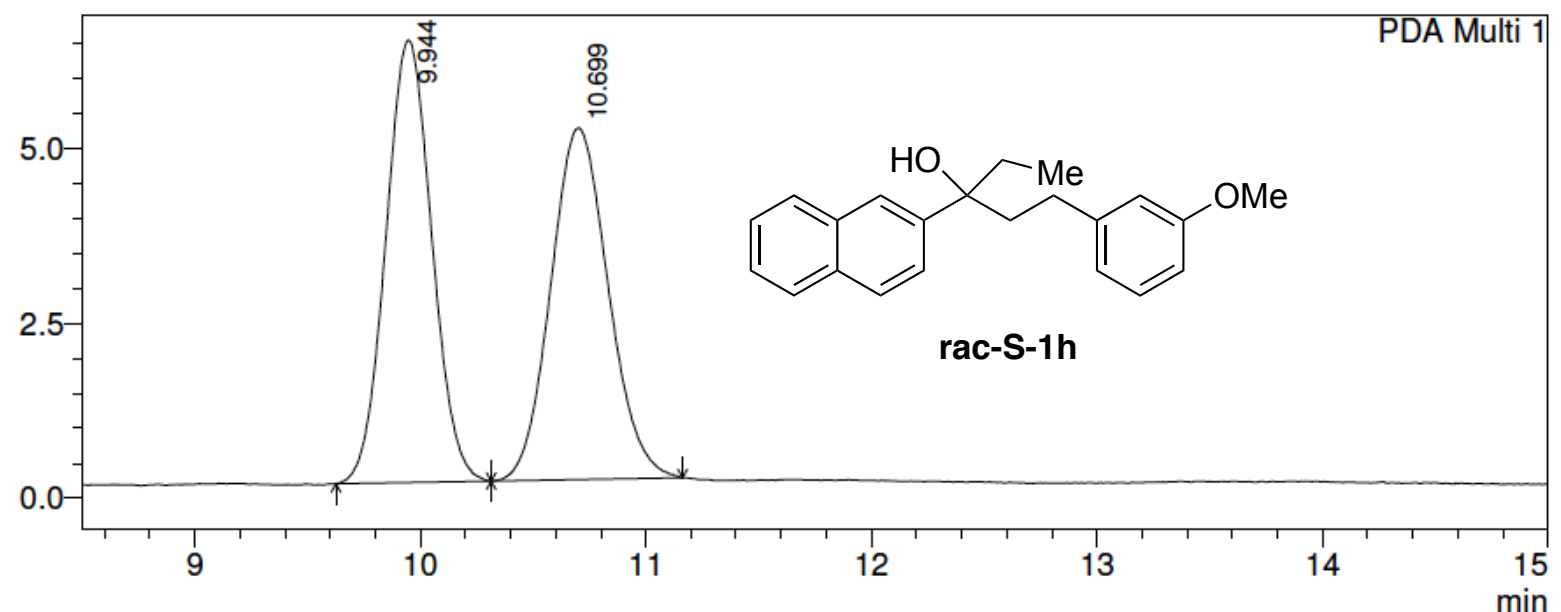

PDA Ch1 254nm 4nm

\begin{tabular}{|r|r|r|r|r|r|}
\hline \multicolumn{1}{|c|}{ Peak\# } & \multicolumn{1}{|c|}{ Ret. Time } & \multicolumn{1}{c|}{ Area } & \multicolumn{1}{|c|}{ Height } & Area \% & Height \% \\
\hline 1 & 9.944 & 89648 & 6313 & 50.174 & 55.701 \\
\hline 2 & 10.699 & 89026 & 5021 & 49.826 & 44.299 \\
\hline Total & & 178674 & 11333 & 100.000 & 100.000 \\
\hline
\end{tabular}

Enantioenriched S-1h, 89\% ee

$\mathrm{mAU}$

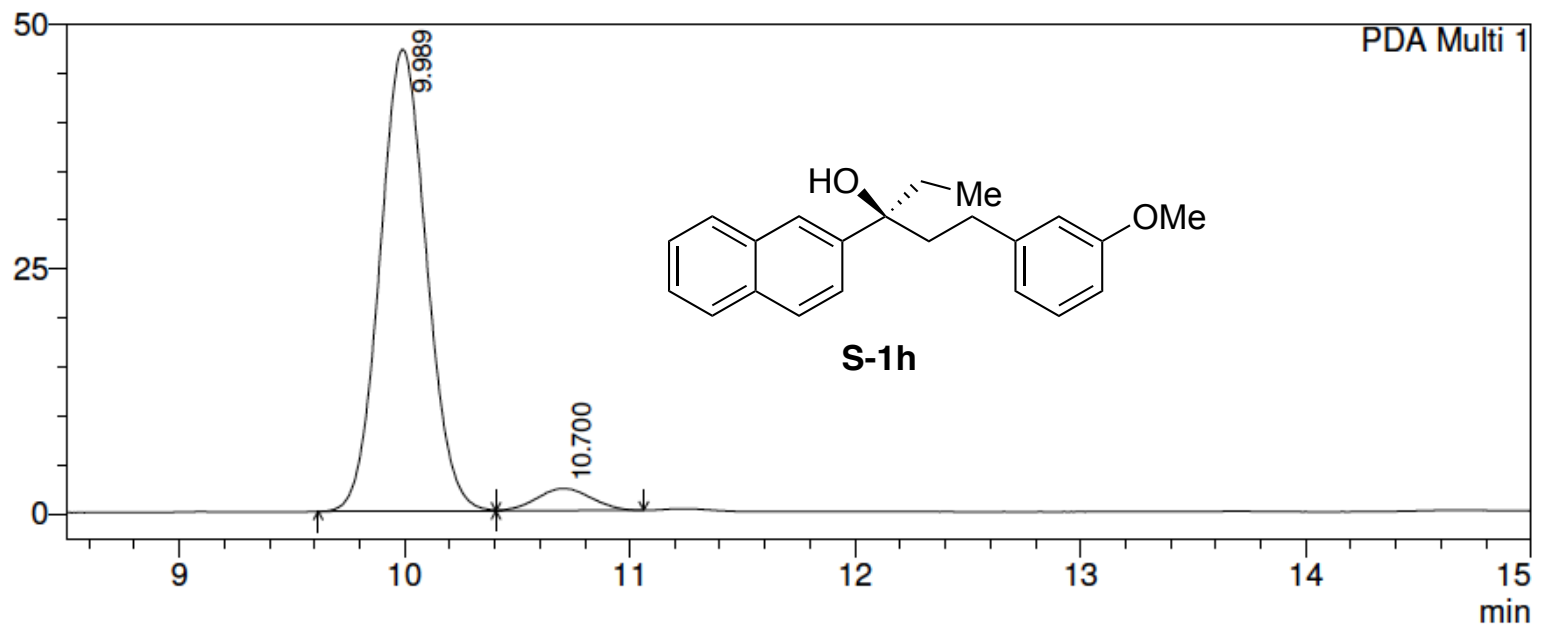

PDA Ch1 254nm 4nm

\begin{tabular}{|r|r|r|r|r|r|}
\hline \multicolumn{1}{|c|}{ Peak\# } & \multicolumn{1}{|c|}{ Ret. Time } & \multicolumn{1}{|c|}{ Area } & \multicolumn{1}{c|}{ Height } & Area \% & \multicolumn{1}{|c|}{ Height \% } \\
\hline 1 & 9.989 & 678937 & 47129 & 94.701 & 95.414 \\
\hline 2 & 10.700 & 37992 & 2265 & 5.299 & 4.586 \\
\hline Total & & 716930 & 49394 & 100.000 & 100.000 \\
\hline
\end{tabular}


Racemic S-1i

$\mathrm{mAU}$

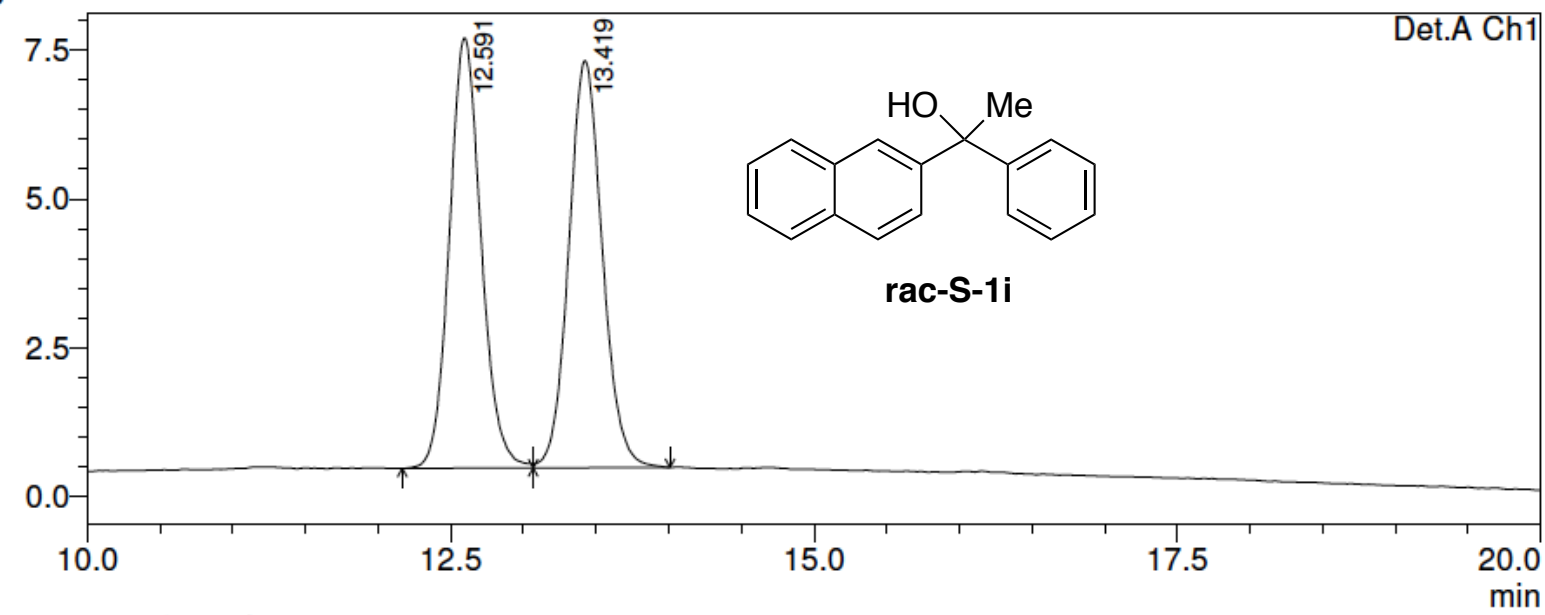

Detector A Ch1 254nm

\begin{tabular}{|r|r|r|r|r|r|}
\hline \multicolumn{1}{|c|}{ Peak\# } & \multicolumn{1}{|c|}{ Ret. Time } & \multicolumn{1}{c|}{ Area } & \multicolumn{1}{|c|}{ Height } & \multicolumn{1}{c|}{ Area \% } & \multicolumn{1}{c|}{ Height \% } \\
\hline 1 & 12.591 & 110150 & 7219 & 50.024 & 51.378 \\
\hline 2 & 13.419 & 110043 & 6832 & 49.976 & 48.622 \\
\hline Total & & 220194 & 14051 & 100.000 & 100.000 \\
\hline
\end{tabular}

Enantioenriched S-1i, $\mathbf{9 6 \%}$ ee $\mathrm{mAU}$

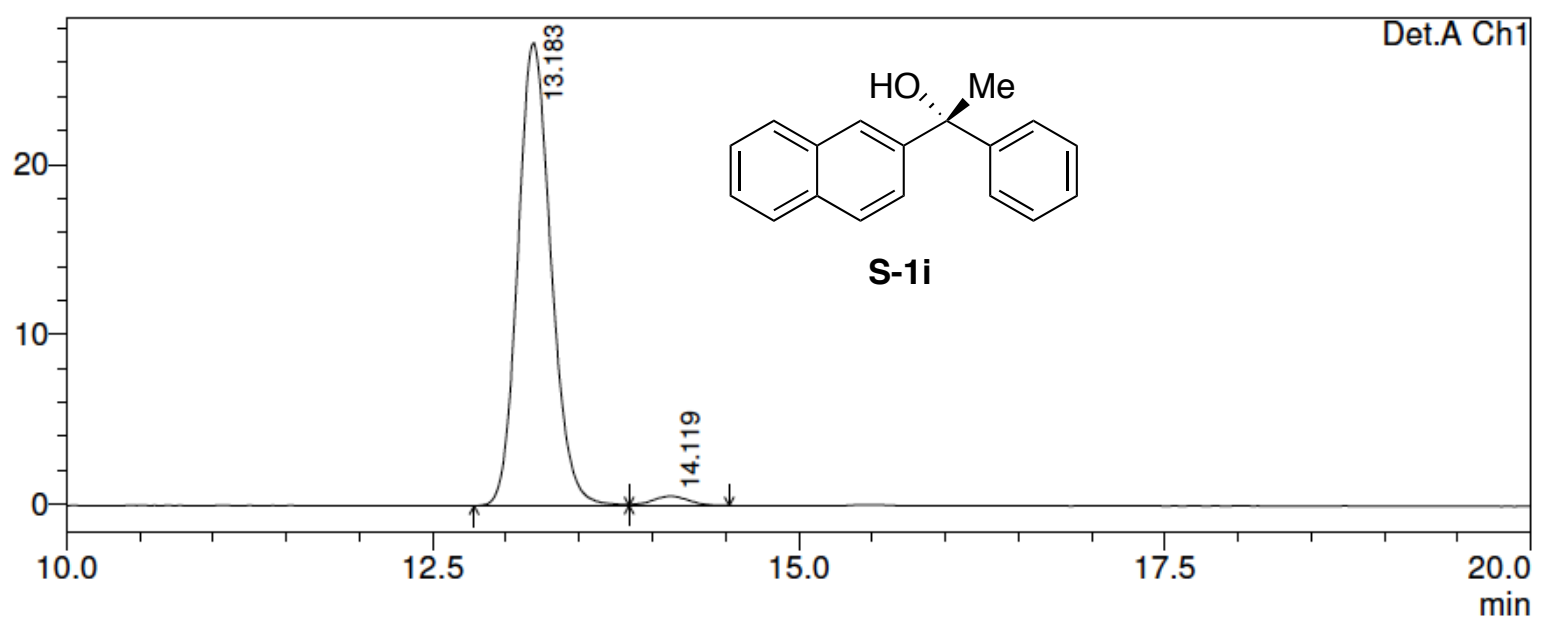

Detector A Ch1 254nm

\begin{tabular}{|r|r|r|r|r|r|}
\hline \multicolumn{1}{|c|}{ Peak\# } & \multicolumn{1}{|c|}{ Ret. Time } & \multicolumn{1}{|c|}{ Area } & \multicolumn{1}{|c|}{ Height } & Area \% & \multicolumn{1}{c|}{ Height \% } \\
\hline 1 & 13.183 & 429320 & 27232 & 97.819 & 98.017 \\
\hline 2 & 14.119 & 9571 & 551 & 2.181 & 1.983 \\
\hline Total & & 438890 & 27783 & 100.000 & 100.000 \\
\hline
\end{tabular}


Racemic S-1j

mAU

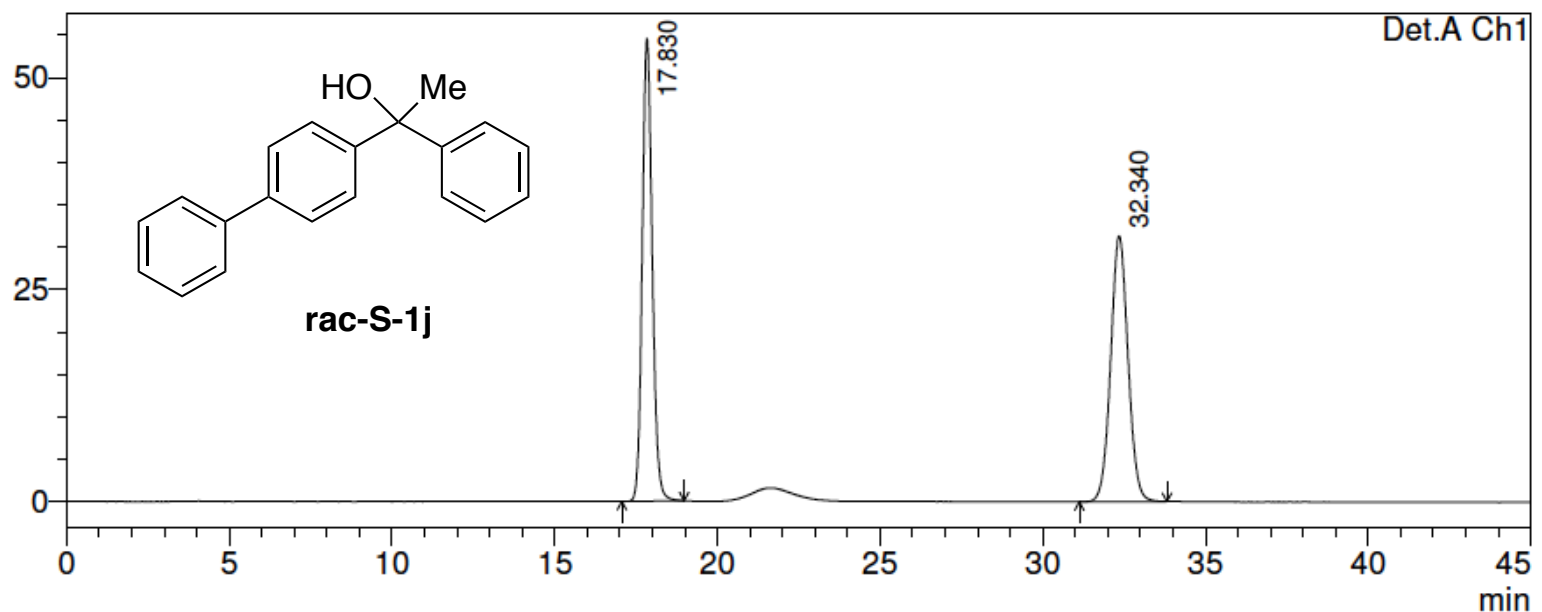

Detector A Ch1 254nm

\begin{tabular}{|r|r|r|r|r|r|}
\hline Peak\# & Ret. Time & \multicolumn{1}{|c|}{ Area } & Height & Area \% & \multicolumn{1}{|c|}{ Height \% } \\
\hline 1 & 17.830 & 1163647 & 54572 & 49.994 & 63.499 \\
\hline 2 & 32.340 & 1163916 & 31370 & 50.006 & 36.501 \\
\hline Total & & 2327563 & 85942 & 100.000 & 100.000 \\
\hline
\end{tabular}

Enantioenriched S-1j, 91\% ee

mAU

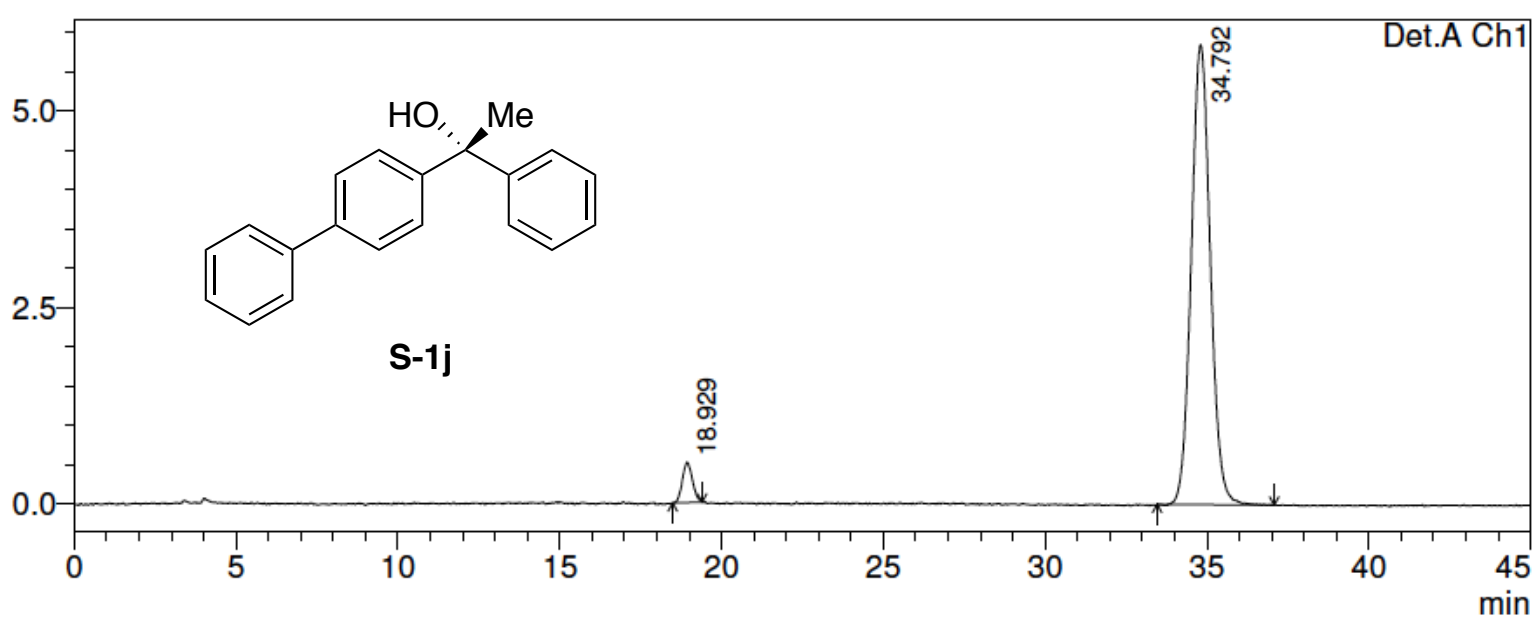

Detector A Ch1 254nm

\begin{tabular}{|r|r|r|r|r|r|}
\hline \multicolumn{1}{|c|}{ Peak\# } & \multicolumn{1}{|c|}{ Ret. Time } & \multicolumn{1}{c|}{ Area } & \multicolumn{1}{c|}{ Height } & \multicolumn{1}{c|}{ Area \% } & Height \% \\
\hline 1 & 18.929 & 11129 & 512 & 4.454 & 8.053 \\
\hline 2 & 34.792 & 238760 & 5845 & 95.546 & 91.947 \\
\hline Total & & 249889 & 6357 & 100.000 & 100.000 \\
\hline
\end{tabular}

